\title{
Ratcheting Up The Search For Dark Matter
}

\author{
by \\ Samuel Dylan McDermott \\ A dissertation submitted in partial fulfillment \\ of the requirements for the degree of \\ Doctor of Philosophy \\ (Physics) \\ in The University of Michigan \\ 2014
}

Doctoral Committee:

Associate Professor Kathryn M. Zurek, Chair

Professor Ratindranath Akhoury

Professor Dante E. Amidei

Assistant Professor David John Baker

Professor James D. Wells 
(C) Samuel Dylan McDermott 2014

All Rights Reserved 


\section{ACKNOWLEDGEMENTS}

Needless to say, this dissertation would never have happened without my fantastic collaborators. My unending gratitude is due to:

- my advisor, Kathryn Zurek (chapters II-VI and VIII)

- Hai-Bo Yu (chapters II-IV)

- Eric Kuflik (chapters V and VI)

- Cliff Cheung (chapter VIII)

- Tomer Volansky and Rouven Essig (chapter VI)

- and Dan Hooper and Ilias Cholis (chapter VII).

On all of these projects, I learned so much from each and every one of these excellent and inspiring physicists. Thank you so much! 
I would additionally like to acknowledge and thank everyone who made this work possible.

Thanks to Fermi National Accelerator Lab, whose generosity with a fifth year predoctoral fellowship allowed me an exceptional opportunity to continue learning.

To the great group of physicists I was lucky to have around at Michigan. Namely: Tim Cohen, Eric Kuflik, and Haibo Yu were the best role models I could have asked for early on; Jack Kearney was the best competition I secretly needed part way through; and Sean Tulin, Rito Basu Thakur, Felix Yu, Ilias Cholis, Andrew Hearin, and Prateek Agrawal were the unbelievably gracious postdocs who allowed me to keep learning till the very end.

My thanks and love are also due, of course, to: my inspiring and stimulating group of friends in Ann Arbor, especially Justin, Mikell, Jon, and Rebecca; to my supportive family; and, more than anyone else, to my lovely girlfriend, Sydney. Your support is the scaffolding that has allowed everything I've done. 


\section{TABLE OF CONTENTS}

ACKNOWLEDGEMENTS ................. ii

LIST OF FIGURES . . . . . . . . . . . . . . . v viii

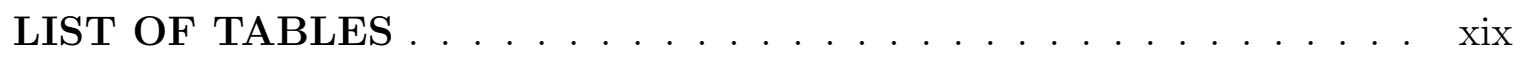

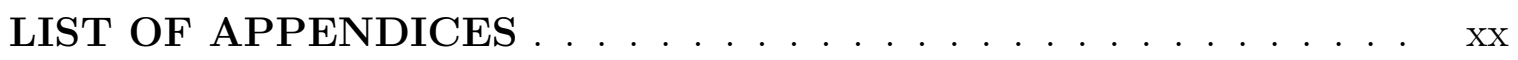

\section{CHAPTER}

I. Introduction $\ldots \ldots \ldots \ldots \ldots \ldots \ldots$

1.1 Dark Matter . . . . . . . . . . . . . . . . . . . 1

1.2 Higgs Physics . . . . . . . . . . . . . . . . . . . . . 10

1.3 Looking Forward . . . . . . . . . . . . . . . . 13

II. Turning Off The Lights: How Dark Is Dark Matter? . . . . . 17

2.1 Introduction . . . . . . . . . . . . . . . . . 18

2.2 Models and General Considerations . . . . . . . . . . 20

2.3 Relic Density Constraints . . . . . . . . . . . . . . . . 21

2.4 Structure Formation and CMB Constraints . . . . . . . . 25

2.4.1 Decoupling at the Recombination Epoch . . . . . 25

2.4.2 Effect on the Dark Matter Virialization . . . . . . . 28

2.5 Dark Matter Halo Constraints . . . . . . . . . . . . . . 30

2.5.1 Elliptical galaxies . . . . . . . . . . . . . . . . . 30

2.5.2 The Bullet Cluster . . . . . . . . . . . . . . . 31

2.6 Direct Detection of Charged Dark Matter . . . . . . . . . 32

2.6.1 Direct Detection Basics . . . . . . . . . . . . . 34

2.6.2 Evacuation of Charged DM from the Disk . . . . . . 35

2.7 Conclusions . . . . . . . . . . . . . . . . . . 37

III. Constraints on Scalar Asymmetric Dark Matter from Black Hole Formation in Neutron Stars . . . . . . . . . . . . 45 
3.1 Introduction . . . . . . . . . . . . . . . . . 46

3.2 Chandrasekhar Limit . . . . . . . . . . . . . . . . . . . 48

3.3 Capture of Asymmetric Dark Matter in Neutron Stars . . . . 50

3.3 .1 Capture rate . . . . . . . . . . . . . . . . . 51

3.3.2 Total Number of ADM in Neutron Stars . . . . . 53

3.4 Asymmetric Scalar Dark Matter in Neutron Stars . . . . . . . 55

3.4 .1 Thermalization . . . . . . . . . . . . . 55

3.4.2 Self-gravitation and Black Hole Formation . . . . . 56

3.4.3 Bose-Einstein Condensation . . . . . . . . . . . . 57

3.5 Hawking Radiation and Destruction of the Host Star . . . . . 59

3.5.1 Black Hole Mass without Bose-Einstein Condensation 61

3.5.2 Black Hole Mass with Bose-Einstein Condensation . 62

3.6 Observational Constraints . . . . . . . . . . . . . 65

3.6.1 Constraints on DM-neutron cross section without Bose-

Einstein condensation . . . . . . . . . . . . 65

3.6.2 Constraints on DM-neutron cross section with BoseEinstein condensation . . . . . . . . . . 67

3.6.3 Constraints from observed pulsars . . . . . . . . . 69

3.7 Conclusions . . . . . . . . . . . . . . . . . . . . 71

\section{The Dark Matter Inverse Problem: Extracting Particle Physics} from Scattering Events _. . . . . . . . . . . . . . 79

4.1 Introduction . . . . . . . . . . . . . . . . . 80

4.2 Preliminaries . . . . . . . . . . . . . . . . . . . . 82

4.3 Methodology . . . . . . . . . . . . . . . . . 86

4.3.1 Detector and Statistical Effects . . . . . . . . . . . 86

4.3.2 Defining the Spectra . . . . . . . . . . . . 88

4.4 Results . . . . . . . . . . . . . . . . . . . . . 90 90

4.4.1 Contour Shapes from Scattering Kinematics . . . . 91

4.4.2 Operator Discrimination with Equal Exposures on All Targets . . . . . . . . . . . . . . . 93

4.4.3 Operator Discrimination with Equal Event Numbers on All Targets . . . . . . . . . . . . . . 100

4.4.4 Comparison of Models . . . . . . . . . . . . . . . 104

4.5 Conclusions . . . . . . . . . . . . . . . . . . 107

V. Neutrino Phenomenology in a $\mathbf{3}+\mathbf{1}+\mathbf{1}$ Framework . . . . . . 112

5.1 Introduction . . . . . . . . . . . . . . . . . . 113

5.2 Phenomenology Of Sterile Neutrino Models . . . . . . . . . 117

5.2 .1 Conventions . . . . . . . . . . . . . . . . . 117

5.2.2 Fits to Neutrino Appearance Anomalies . . . . . . . 119

5.3 Phenomenology of the Heavy Neutrino $n_{5}$ in the $3+1+1$ Scheme 126 
5.3.1 The Gallium and Reactor Anomalies _. . . . . . 126

5.3.2 Big Bang Nucleosynthesis . . . . . . . . . . . . . . . 128

5.3 .3 Supernova 1987A . . . . . . . . . . . . . . . . . . 132

5.3.4 Bounds from Light Mesons . . . . . . . . . . . 133

5.3.5 Combined Bounds on $n_{5} \ldots \ldots \ldots \ldots$

5.4 Neutrino Models . . . . . . . . . . . . . . . . . . . . . . 139

5.5 Conclusions . . . . . . . . . . . . . . . . . . . . . . . 141

VI. Constraining Light Dark Matter with Diffuse X-Ray and GammaRay Observations . . . . . . . . . . . . . . . . . . . 149

6.1 Introduction . . . . . . . . . . . . . . . . 150

6.2 Constraining Light Dark Matter with Diffuse Photons . . . . 152

6.2.1 Flux from Dark Matter Decays and Annihilations . 153

6.2 .2 Data . . . . . . . . . . . . . . . . . 155

6.2 .3 Statistical Methodology . . . . . . . . . . . . . 157

6.3 Models of Decaying Light Dark Matter . . . . . . . . . . . . 158

6.3 .1 Hidden Photino . . . . . . . . . . . . . . . . . . 158

$6.3 .2 \quad$ Sterile Neutrino . . . . . . . . . . . . . . . . . . . 161

6.3.3 Gravitino Dark Matter . . . . . . . . . . . . . . . 164

6.3 .4 Dipole DM . . . . . . . . . . . . . . . . . . . . . . 167

6.3.5 Dark (Pseudo-) Scalars . . . . . . . . . . . . . 167

6.4 Model-Independent Bounds and Spectra . . . . . . . . . . 168

6.4.1 Two-Body Decays Involving a Photon . . . . . . . . 169

6.4 .2 Two-Body Decays with FSR . . . . . . . . . . 170

6.4.3 Two-Body Cascade Decays . . . . . . . . . . . . 172

6.4.4 Three-Body Decays with FSR . . . . . . . . . . . 173

6.4.5 Three-Body Decays Involving Photons . . . . . . . . 174

6.5 Annihilating Light Dark Matter . . . . . . . . . . . . 175

6.6 Conclusions and Future Improvements . . . . . . . . . . 178

VII. Dissecting the Gamma-Ray Background in Search of Dark Matter . . . . . . . . . . . . . . . . . . . . . . 189

7.1 Introduction . . . . . . . . . . . . . . . . . . 190

7.2 Astrophysical Contributions to the Diffuse Gamma-Ray Background . . . . . . . . . . . . . . . . . . . . . . 192

7.2.1 Star-Forming Galaxies _. . . . . . . . . . . 193

7.2 .2 Radio Galaxies . . . . . . . . . . . . . . . . . . 196

7.2 .3 Blazars . . . . . . . . . . . . . . . . . . . 197

7.2.4 Ultra-High Energy Cosmic Ray Propagation . . . 197

7.2 .5 Millisecond Pulsars . . . . . . . . . . . . . . . . . 199

7.2 .6 Other Contributions . . . . . . . . . . . . . . . . 201

7.2.7 The Combined Astrophysical Contribution to the Extragalactic Gamma-Ray Background . . . . . . . . . 203 
7.3 Gamma Rays from Annihilating Dark Matter . . . . . . . . . 204

7.3.1 The Extragalactic Contribution . . . . . . . . . . 204

7.3.2 The Smooth Galactic Halo . . . . . . . . . . . . 208

7.3.3 Subhalos of the Milky Way . . . . . . . . . . . . . 209

7.4 Constraints on the Dark Matter Annihilation Cross Section . 213

7.5 Projections And Future Sensitivity . . . . . . . . . . . . 215

7.6 Summary and Conclusions _. . . . . . . . . . . 217

VIII. Inspecting the Higgs for New Weakly Interacting Particles . 229

8.1 Introduction . . . . . . . . . . . . . . . . . . 230

8.2 Framework . . . . . . . . . . . . . . . . . 233

8.2 .1 Theory Parameterization . . . . . . . . . . . 233

8.2.2 Observables Parameterization _ . . . . . . . . . . . 237

8.3 Tree Level Effects . . . . . . . . . . . . . . . . . . . . . . . . 239

8.4 Loop Level Effects . . . . . . . . . . . . . . . . . . . . 244

8.5 NMSSM . . . . . . . . . . . . . . . . . 252

8.5.1 Masses And Kinematics . . . . . . . . . . . . . 253

8.5.2 Signal Strengths . . . . . . . . . . . . . . . 255

8.6 Conclusions . . . . . . . . . . . . . . . . . . . 259

IX. Conclusion $\ldots \ldots \ldots \ldots \ldots \ldots \ldots \ldots \ldots \ldots$

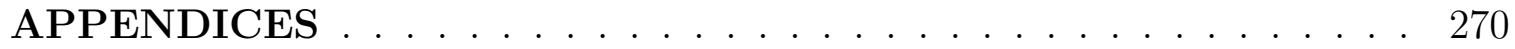




\section{LIST OF FIGURES}

\section{Figure}

2.1 Constraints from various sources, from top to bottom: (i) Scattering in the bullet cluster and NGC720, (ii) DM as a charged thermal relic, and (iii) DM virial processes, and (iv) recombination epoch. . . . .

2.2 CoGeNT (blue), DAMA (green) allowed regions at 99\% C.L. The CDMS-Si (yellow) line is included as a sample exclusion at 99\% C.L. In the gray area, the charged DM is evacuated from the Galactic disk. Also shown the bound from Recombination epoch (red). Below the dotted line (black), charged DM may diffuse to the disk. . . . . . .

3.1 Regions (gray) of DM-neutron scattering cross-section in which accumulated scalar ADM forms a black hole. We have $\rho_{X}=0.1 \mathrm{~cm}^{3} / \mathrm{s}$ along the dotted red lines, which is approximately the DM density in the solar neighborhood. In the left panel, we assume a Bose-Einstein condensate (BEC) does not form and all captured DM particles become self-gravitating and collapse. In the right panel, we assume a Bose-Einstein condensate (BEC) does form and DM particles in the BEC ground state collapse and form a black hole. We take the neutron star age to be $10^{10}$ years with a central temperature $10^{5} \mathrm{~K}$. In the diagonally shaded regions, DM particles cannot thermalize with neutrons within the age of neutron star. In the diagonally crosshatched region, the black hole can evaporate due to the Hawking radiation. In the square-hatched regions, Hawking radiation may interfere with DM accretion by heating the thermalized DM, and in some cases the black hole can evaporate (see discussion in Section V). In these hatched regions, the bounds are lifted. The black regions are excluded by recent CDMS results (SI) [3] . . . . . . . . . . . . .

3.2 Regions (colored) excluded by the nearby pulsars J0437-4715 (left) and J2124-3358 (right). The shaded, diagonal and square crosshatched, and black regions are as in Fig. (3.1). In hatched regions, the bounds are lifted. . . . . . . . . . . . . . . . . . . . 
3.3 Regions (colored) excluded by the pulsar B1620-26 in the globular cluster M4. Note the globular cluster M4 is a baryon-dominated system, and there is no evidence for the presence of dark matter. Here, we take $\rho_{X}=10^{3} \mathrm{GeV} / \mathrm{cm}^{3}$ motivated by numerical results in Refs. [25, 38]. The shaded, diagonal and square cross-hatched, and black regions are as in Fig. (3.1). In hatched regions, the bounds are

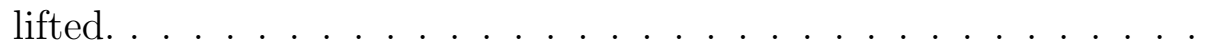

4.1 95\% CLCs for a 10, 50, and $250 \mathrm{GeV}$ particle interacting through an $n_{i}=$ standard operator with equal exposures, such that there are 300 events on a germanium target and more (fewer) on a xenon (argon) target. Comparisons are made to $\nu_{i}=$ standard, $q^{4}$, and $q^{-4}$ operators. The colors represent the value of $\widetilde{L}_{\min } /$ d.o.f. The standard interaction can be distinguished from the $q^{ \pm 4}$ operators via both CLC overlap and the values of $\widetilde{L}_{\min } /$ d.o.f. As can be seen in Fig. (10) in the appendix, $\widetilde{L}_{\min } /$ d.o.f. is less powerful for distinguishing $n_{i}=$ standard from $\nu_{i}=q^{ \pm 2}$, though overlap remains robust. . . . . . . .

4.2 As in Fig. 4.1, but with an $n_{i}=$ dipole operator compared to $\nu_{i}=$ standard, $q^{2}$, and $q^{4}$ operators. . . . . . . . . . . . . . .

4.3 Effect of momentum dependence and DM mass on fit spectra. The data are from an $n_{i}=$ standard interaction and are depicted with Poisson error bars. The best fit spectrum for $\nu_{i}=$ standard is shown as a green solid line. We also show two $\nu_{i}=q^{4}$ spectra. The $q^{4}$ spectrum in blue is at the best fit point and the $q^{4}$ spectrum in red is taken at high mass to illustrate the improvement in goodness of fit from reducing $m_{\mathrm{DM}}$. We can also see the effect of xenon's higher event rate and lower energy threshold on its ability to determine the correct operator. . . . . . . . . . . . . . . . . . .

4.4 Effect of momentum dependence and DM mass on fit spectra. The data are from an $n_{i}=$ standard interaction and are depicted with Poisson error bars. The best fit spectrum for $\nu_{i}=$ standard is shown as a green solid line. We also show two $\nu_{i}=q^{-4}$ spectra. The $q^{-4}$ spectrum in blue is at the best fit point and the $q^{-4}$ spectrum in red is taken at low mass to illustrate the improvement in goodness of fit from increasing $m_{\mathrm{DM}}$. We can also see the effect of xenon's higher event rate and lower energy threshold on its ability to determine the correct operator. . . . . . . . . . . . . . . . . . . 
4.5 Fits of $\nu_{i}=\operatorname{std}, q^{2}$, and $\mathrm{q}^{4}$ operators to $n_{i}=$ dipole data. The data are shown as points with Poisson error bars. The dipole best fit spectrum is shown as a solid line and the other operator best fit spectra are shown as dotted lines. Xenon is red, germanium is blue, and argon is green. . . . . . . . . . . . . . . .

4.6 95\% CLCs for 10, 50, and $250 \mathrm{GeV}$ particles interacting through an $n_{i}=$ standard operator with 300 events on each target. Comparisons are made to $\nu_{i}=$ standard, $q^{4}$, and $q^{-4}$ operators. Colors as in Fig. 4.1.101

4.7 $95 \%$ CLCs for a 10, 50, and $250 \mathrm{GeV}$ particles interacting through an $n_{i}=$ dipole moment operator with 300 events on each target. Comparisons are made to $\nu_{i}=$ standard, $q^{2}$, and $q^{4}$ operators. The colors represent the value of $\widetilde{L}_{\min } /$ d.o.f. Compared to the case shown in Fig. (4.2) where exposures were fixed for all targets so that xenon was able to power the statistics, the ability to discriminate the dipole interaction from standard and $q^{4}$ operators using overlap or the values of $\widetilde{L}_{\min } /$ d.o.f. is diminished.

4.8 The p-values of trial operators for $n_{i}=$ standard and $q^{2}$ operators for candidate masses of 50 and $250 \mathrm{GeV}$. We display the equal exposure and equal event bar charts side by side to underscore the robustness of the discrimination. For the visual purpose, the plot is normalized so that each bar starts at $5 \%$ significance level. . . . . . . . . . .

4.9 The $\mathrm{p}$-values of trial operators for $n_{i}=$ anapole, dipole, and $q^{-4}$ operators for candidate masses of 50 and $250 \mathrm{GeV}$. We display the equal exposure and equal event bar charts side by side to underscore the robustness of the discrimination. For the visual purpose, the plot is normalized so that each bar starts at 5\% significance level. . . .

5.1 Comparison of 2010 (upper panel) and 2011 (lower panel) MB $\bar{\nu} L / E$ data with $\mathrm{MB} \nu$ and LSND $\bar{\nu} L / E$ data. In both panels, the MB $\nu$ data is taken from [4]. In the upper panel, the MB $\bar{\nu}$ data is taken from [5], while in the lower panel the MB $\bar{\nu}$ data has been updated with the results of [8]. We show the best fit lines in the $3+1$ scenario (black), the $3+1+1$ scenario (green) using all data points, and the $3+1+1$ scenario (orange) dropping the three low-energy data points so that the data is in the range $E_{\nu}>475 \mathrm{MeV}$. In all plots, $\nu$ lines are dotted and $\bar{\nu}$ lines are solid. 
5.2 Fits in the $3+1$ (left) and $3+1+1$ (right) neutrino models. We also contrast the allowed regions using the 2010 (light-orange) and the 2011 (dark-orange) MB $\bar{\nu}$ data. In both panels we show the appearance allowed region at $99 \%$ as well as the appearance null result and disappearance null result exclusion curves at 99\%. There is significant tension with the disappearance experiments and oscillations reported by LSND and MB for both the $3+1$ and $3+1+1$ scenarios with the 2011 data. . . . . . . . . . . . . . . . . . . .

5.3 Constraints on $r$ as a function of $\left|U_{e 4}\right|^{2}\left|U_{\mu 4}\right|^{2}$ for $0.04 \mathrm{eV}^{2}<\Delta m_{41}^{2}<$ $6 \mathrm{eV}^{2}$. We see that $r$ is close to 1 in the appearance preferred region, and has limited ability to reduce the tension with null experiments.

$5.4 \quad \chi^{2}$ as a function of the CP-odd parameter $\beta$, with (left) and without (right) the $\mathrm{MB} \nu$ and $\bar{\nu}$ data for $200 \mathrm{MeV}<E_{\nu}<475 \mathrm{MeV}$. We show fits utilizing both the 2010 and $2011 \mathrm{MB} \bar{\nu}$ data, and we show the $90 \%$ or $99 \%$ allowed value from the $\Delta \chi^{2}$ test that we use. . . . . .

5.5 Exclusion regions from BBN [34] (right frame) and SN1987A [96] (both frames), as well as bounds from the $\mathrm{NuTeV}$ oscillation search [16] (red dotted, right frame), $R_{\pi}[37,38]$ (left frame), measurements of $\tau_{\mu}$ [38-42] (left frame), collider and line searches [38,43-49] (both frames), and searches for $\mu \rightarrow e \gamma$ [50] (both frames). The left panel shows lines of constant values of $r$ from 1.05 to 2.4 (for the calculation of $r$, we assume no CP violation and take $\left|U_{e 4} U_{\mu 4}\right|=0.023$, as explained in the text). To avoid clutter, we avoid repeating the $\tau_{\mu}$ and $\mathrm{NuTeV}$ lines in both plots, although each is valid in both cases.

6.1 The collected normalized dataset of photon fluxes used to place constraints on decaying and annihilating DM in this paper. Data from HEAO-1 [63] (orange), INTEGRAL [64] (green), COMPTEL [65] (blue), EGRET [66] (red), and Fermi [67] (yellow) are shown. All error bars are statistical, except for the EGRET and Fermi datasets, where the dominant systematic uncertainties are shown. We omit the INTEGRAL $511 \mathrm{keV}$ line both in this figure and in our analysis. Note that the various datasets span different regions of the sky and should therefore not be compared with each other; they appear together on this plot only for convenience. . . . . . . . . . . 156 
6.2 Constraints on hidden photino decay to left: gravitino and photon and right: gravitino and hidden photon (with the latter taken to have mass $m_{\gamma_{d}}=0.9 m_{\widetilde{\gamma}_{d}}$ and going to final state $f^{+} f^{-}$, with $f=$ $e, \mu$ or $\pi$ ). In the left plot, the solid (dotted) lines are with $\sqrt{F}=$ $10^{4}\left(10^{2}\right) \mathrm{TeV}$. The constraints are derived from the diffuse gammaand X-ray data taken from HEAO-1 (orange), INTEGRAL (green), COMPTEL (blue), EGRET (red), and Fermi (yellow). In the "ShortLived" region the DM lifetime is shorter than the age of Universe. Above the solid red line, the hidden photino is stable. . . . . . . .

6.3 Constraints on a hidden photon in the hidden photino DM model for the case where the hidden photino decays to a photon and a gravitino, $\tilde{\gamma}_{d} \rightarrow \gamma \tilde{G}$, and with $\sqrt{F}=100 \mathrm{TeV}$ (left) or $\sqrt{F}=10^{4} \mathrm{TeV}$ (right). Gray shaded regions indicate constraints from beam-dump, fixedtarget, and colliding beam experiments, stars, precision measurements, and from the intergalactic diffuse photon background (IDPB), while the colored regions show the gamma- or X-ray constraints as in Fig. 6.2. In the "Short-Lived" region the DM lifetime is shorter than the age of Universe. See text for more details. . . . . . . . . . . .

6.4 Decay channels for a sterile neutrino, $\nu_{s}$, through (A) a two-body radiative process $\left(\nu_{s} \rightarrow \nu_{\alpha} \gamma\right)$ and (B) charge- and neutral-current contributions to a three-body final state. . . . . . . . . . . . . . .

6.5 Constraints on the sum of sterile-neutrino decay to $\gamma \nu$ and $\nu e^{+} e^{-}$ using the decay widths in Eqs. (6.11) and (6.12). The constraints from the diffuse gamma- and X-ray data are HEAO-1 (orange), INTEGRAL (green), COMPTEL (blue), and EGRET (red). Within the solid black region, the neutrino energy density must be greater than the observed DM density. Above (below) the black solid line, the neutrino lifetime is shorter (longer) than the age of the Universe. Within the green boundaries, the sterile neutrino is ruled out by Ly- $\alpha$ forest data $[48,49]$. Two cases for the sterile-neutrino energy density are assumed. In the left plot, the density is assumed to precisely equal the DM energy density everywhere below the dark and light gray regions. In the right plot, the density is determined by the (irreducible) DW mechanism. . . . . . . . . . . . . . . .

6.6 Feynman diagrams for $\widetilde{G}$ decay through (A) an off-shell slepton to a three-body final state $\left(\widetilde{G} \rightarrow \ell_{i}^{ \pm} \nu_{j} \ell_{k}^{\mp}\right)$ and (B) a two-body radiative process $\left(\widetilde{G} \rightarrow \nu_{\alpha} \gamma\right) \ldots \ldots \ldots \ldots \ldots$ 
6.7 Left: Constraints on photino-neutrino mixing from RPV gravitino decay. Right: Constraints on the effective cutoff scale for DM with a dipole interaction. Regions as in Fig. 6.2. . . . . . . . . . . . . .

6.8 Constraints on the decay constant $f_{\pi_{d}}$ for a dark pseudoscalar decaying to diphotons (left) and the limits on the coupling of a hidden scalar in the case where it decays to $e^{+} e^{-}$(right). Regions as in Fig. 6.2. . . . . . . . . . . . . . . . . . . 168

6.9 Bounds on the lifetime of a scalar DM, $\phi$, decaying to two photons. Regions as in Fig. 6.2. . . . . . . . . . . . . . . . . 170

6.10 Left: Photon spectra from DM decay to $e^{+} e^{-}$, emitting final state radiation, as a function of $x=2 E_{\gamma} / m_{\mathrm{DM}}$. The spectrum of decays with galactic photons only is shown as the solid line, while the redshifted extragalactic spectrum is shown with dashed lines (see text for details). Right: Bounds on the DM decay lifetime for this process, with regions as in Fig. 6.2. . . . . . . . . . . . . . . .

6.11 Left: Photon spectra versus $x=2 E_{\gamma} / m_{\text {DM }}$ for DM decay to two neutral particles, where one of the neutral particles subsequently decays to $e^{+} e^{-}$, emitting final state radiation. The lines are as in Fig. 6.10. Right: Bounds on the DM decay lifetime for this process. Regions are as in Fig. 6.2. . . . . . . . . . . . . . . . . .

6.12 Left: Photon spectra versus $x=2 E_{\gamma} / m_{\mathrm{DM}}$ for DM decay to $e^{+} e^{-} \nu$, emitting final state radiation. The lines represent the galactic (solid) and extragalactic (dashed) spectra. Right: Bounds on the DM decay lifetime for this process. Regions as in Fig. 6.2. . . . . . . . . . 173

6.13 Left: Photon spectra versus $x=2 E_{\gamma} / m_{\mathrm{DM}}$ for DM three-body decay $\phi_{1} \rightarrow \phi_{2} \gamma \gamma$. The lines represent the galactic (solid) and redshifted extragalactic (dashed) spectra. Right: Bounds on the DM decay lifetime for this process, with regions as explained in Fig. 6.2 . . . . 175

6.14 Bounds on the DM velocity-averaged annihilation cross-section $\langle\sigma v\rangle$ due to FSR off the process $\chi \chi \rightarrow e^{+} e^{-}$. Regions as in Fig. 6.2. Also shown is a comparison with the CMB constraint for DM annihilation that is $s$-wave (solid) or $p$-wave, the latter for two different kinetic-decoupling temperatures, $x_{\mathrm{kd}} \equiv T_{\gamma} / m_{\mathrm{DM}}=10^{-4}$ (dash-dot) and $10^{-6}$ (dashed line), where we take $T_{\gamma}=0.235 \mathrm{eV}$ at the CMB epoch (corresponding to $\left.z_{\mathrm{CMB}}=1000\right) \ldots \ldots \ldots \ldots$ 
7.1 The models used in our analysis to describe the spectral shape of the gamma-ray emission from Milky Way-like star-forming galaxies (left) and much higher luminosity starburst galaxies (right), neglecting attenuation from the cosmic infrared background. See text for details. . . . . . . . . . . . . . . . . . . .

7.2 The estimated contribution to the EGB from star-forming galaxies (including starburst galaxies). The dashed curve represents the estimate derived using the central parameter values, while the solid lines are the $1 \sigma$ uncertainties around that prediction. The error bars denote the spectrum of the EGB as measured by Fermi [3], while the points without error bars are the central values of the Fermi's preliminary EGB analysis, currently in preparation and shown only for comparison [47]. See text for details. . . . . . . . . . . . . .

7.3 The estimated contribution to the EGB from radio galaxies (including both FRI and FRII galaxies). The dashed curve represents the estimate derived using the central parameter values, while the solid lines are the $1 \sigma$ uncertainties around that prediction. Error bars and points are as in Fig. 7.2. See text for details. . . . . . . . . . . . .

7.4 The estimated contribution to the EGB from blazars (BL Lacs and FSRQs). Once again, the dashed curve represents the estimate derived using the central parameter values, while the solid lines are the $1 \sigma$ uncertainties around that prediction. Error bars and points are as in Fig. 7.2. See text for details. . . . . . . . . . . . . . .

7.5 The estimated contribution to the EGB from the propagation of ultra-high energy cosmic rays. The upper and lower sets of curves correspond to models with very strong source evolution and no source evolution, respectively [35]. Error bars and points are as in Fig. 7.2. See text for details. . . . . . . . . . . . . . . . . . . . . . . 198

7.6 The estimated contribution to the high-latitude, diffuse gamma-ray background from millisecond pulsars. See text for details. . . . . . . 200

7.7 The estimated contribution to the EGB from the combination of radio galaxies, star-forming galaxies, and blazars (FSRQs and Bl Lacs). The dashed contour represents the prediction using central values for all model parameters. The solid contours are the $1 \sigma$ uncertainties around this prediction, after propagating all parameter uncertainties. Error bars and points are as in Fig. 7.2. See text for details. . . . . 202

7.8 Two examples of viable models which provide a good fit to the observed EGB. See text for details. . . . . . . . . . . . . . . . . 202 
7.9 The extragalactic dark matter annihilation contribution to the EGB for a reference dark matter model $\left(m_{\mathrm{DM}}=100 \mathrm{GeV}\right.$, annihilating to $b \bar{b}$ with $\sigma v=3 \times 10^{-26} \mathrm{~cm}^{3} / \mathrm{s}$ ). The upper curve is the result using the substructure boost factor of Eq. 7.9, which is based on an extrapolation of numerical simulations. The dotted curve assumes a boost factor that is a factor of 30 lower than our default model. The lowest curve neglects the contribution from substructure entirely. See text for details. . . . . . . . . . . . . . . . . . 206

7.10 The halo mass function, $d n / d M$, and the integral of the $(1+z)^{-1}$ weighted halo mass function using the model of Tinker et al. [76] (adopted in our calculations) and the ellipsoidal collapse model adopted in Ref. [73]. We also show results using pre-Planck (dashed) and post-Planck (solid) values for the relevant cosmological parameters. These differences have only a modest impact on the contribution of dark matter annihilations to the extragalactic gamma-ray background.208

7.11 The contribution to the extragalactic gamma-ray background from dark matter annihilations in the smooth halo of the Milky Way, for a reference dark matter model $\left(m_{\mathrm{DM}}=100 \mathrm{GeV}\right.$, annihilating to $b \bar{b}$ with $\sigma v=3 \times 10^{-26} \mathrm{~cm}^{3} / \mathrm{s}$ ). The result has been averaged over the following region of the sky: $0<\ell<2 \pi$ and $|b|>30^{\circ}$. See text for details. . . . . . . . . . . . . . . . . . . . .

7.12 The contribution to the EGB from subhalos of the Milky Way, for a reference dark matter model $\left(m_{\mathrm{DM}}=100 \mathrm{GeV}\right.$, annihilating to $b \bar{b}$ with $\sigma v=3 \times 10^{-26} \mathrm{~cm}^{3} / \mathrm{s}$ ). The upper curve is the result using our default model, while the lower dotted curve is reduced by a factor of 30 relative to our default model. See text for details. . . . . . . . . .

7.13 The total contribution from dark matter annihilations to the EGB, for a reference dark matter model $\left(m_{\mathrm{DM}}=100 \mathrm{GeV}\right.$, annihilating to $b \bar{b}$ with $\sigma v=3 \times 10^{-26} \mathrm{~cm}^{3} / \mathrm{s}$ ). The upper curve is the result using our default substructure model, while the lower dotted curve is reduces the contribution from substructure by a factor of 30 relative to our default model. See text for details. . . . . . . . . . . . . .

7.14 Our model of the EGB, including the largest allowed contribution from annihilating dark matter (at the 95\% CL). Here, we have adopted our default substructure model. In each case, we have marginalized over the parameters of our astrophysical model. See text for details. 
7.15 In the left frame, we show the limits (95\% CL) on the dark matter annihilation cross section derived in this study, using our default substructure model (solid), and neglecting substructure (dashes). In the right frame, we compare this result to the strongest existing constraints on the dark matter annihilation cross section from observations of the Galactic Center [37] and of dwarf spheroidal galaxies [38]. See text for details. . . . . . . . . . . . . . . .

7.16 Projected uncertainties for an astrophysical model of the extragalactic gamma-ray background, after ten years of data from Fermi. In the left frame, we compare this model to the preliminary Fermi measurement [47], whereas in the right frame we compare it to the measurement projected with ten years of data. See text for details. . . .

7.17 Our projected sensitivity to dark matter annihilation from Fermi measurements of the EGB after 10 years of operation, using the astrophysical model and projected error bars as shown in the right frame of Fig. 7.16. See text for details. . . . . . . . . . . . . . . . 217

8.1 Contours of $\tan \beta$ (left) and $\tan \delta$ (right) as functions of $\left(R_{i}^{g} / R_{i}^{V}, R_{b}^{j} / R_{V}^{j}\right)$, which are obtained directly from experiment. The red shaded regions with solid (dashed) borders show values that will remain consistent with the SM with $300(3000) \mathrm{fb}^{-1}$ and blue shaded regions show values which cannot be observed within this framework. The solid (dotted) curves show the region where $d_{b} / d_{V}$ is positive (negative). .

8.2 Contours of $R_{b}^{j} / R_{V}^{j}$ (left) and $R_{i}^{g} / R_{i}^{V}$ (right) as a function of theory parameters $(\delta, \beta)$. The red shaded regions are as in Fig. 8.1. . . . 242

8.3 Contours of $R_{i}^{j} / \cos ^{2} \gamma$ (which we approximate by $\left.R_{i}^{j}\right|_{\gamma=0}$ ) for $V$ (top row) and $b$ (bottom row) final states from a variety of production channels. Once $\delta$ and $\beta$ are determined from observation, measuring $R_{i}^{j}$ can then be used to obtain $\gamma \ldots \ldots \ldots \ldots \ldots$

8.4 Contours of $R_{\gamma}^{j} / R_{V}^{j}$ in the plane of theoretical parameters $(\delta, \beta)$, with no new charged particles beyond the SM present. The red shaded regions are as in Fig. 8.1. . . . . . . . . . . . . . . . . . 245 
8.5 Taking the low $\tan \beta$ limit, we plot the new physics contribution to the diphoton rate as a function of $R_{\gamma}^{j} / R_{V}^{j}$ and $R_{i}^{g} / R_{i}^{V}$. The left (right) panel shows values of $\sum_{\mathrm{i} \notin \mathrm{SM}} \mathcal{A}_{\gamma, i} d_{i} / d_{V}$ for which $d_{\gamma} / d_{V}$ is positive (negative). In both panels, solid (dotted) lines show values of $\sum_{\mathrm{i} \notin \mathrm{SM}} \mathcal{A}_{\gamma, i} d_{i} / d_{V}$ for which $d_{g} / d_{V}$ is positive (negative). The red shaded regions are as in Fig. 8.1. We use the uncertainty in $R_{i}^{g} / R_{i}^{V}$ rather than $R_{i}^{t} / R_{i}^{V}$ since the former will be much better measured, as shown in Tab. 8.2. . . . . . . . . . . . . . . . . .

8.6 Contours of $\eta_{s, \psi}$ as a function of $\left(R_{\gamma}^{j} / R_{V}^{j}, \gamma\right)$, in the decoupling limit $\delta=0$. The red shaded regions are as in Fig. 8.1. . . . . . . . . 248

8.7 Contours of $R_{\gamma}^{j} / R_{V}^{j}$ as a function of $(\delta, \gamma)$ for fixed values of $\tan \beta$ and $\eta_{s, \psi}$ for a unit-charged fermion, and $\eta_{d, \psi}=\eta_{u, \psi}=0 . \ldots 251$

8.8 Contours of constant $R_{\gamma}^{j} / R_{V}^{j}$ for fixed values of $\tan \beta$ using a single fermion loop particle that couples only to the singlet component of the Higgs. We take $\gamma=0.2(0.4)$, which represents the $68 \%(95 \%)$ confidence level limits on the amount of the singlet in the lightest physical Higgs. The pinching behavior should occur in the left panel as well, but requires $\left|\eta_{s, \psi}\right|>2 . \ldots \ldots \ldots \ldots \ldots \ldots$

8.9 Contours of constant $m_{\chi_{1}^{ \pm}}$for fixed $\bar{m}$. Regions that fail LEP bounds are shaded red. $\ldots \ldots \ldots \ldots \ldots \ldots \ldots$

8.10 Contours of (left) $R_{V}^{g}$ and (right) $R_{b}^{g}$ for $\tan \beta=1 \ldots \ldots 256$

8.11 Contribution from the $\chi^{ \pm}$loop in the decoupling limit with $\gamma=$ $0.2,0.4$ for (left) $\bar{m}=150 \mathrm{GeV}$ and (right) $\bar{m}=250 \mathrm{GeV}$. In the shaded red regions, $m_{\chi_{1}^{ \pm}} \leq 103.5 \mathrm{GeV}$ for $\lambda=0.7 \ldots \ldots \ldots 258$

8.12 Same as Fig. 8.11 but in the $\lambda M_{2}-\bar{m}$ plane and with $\tan \beta=1 . .258$

A.1 $95 \%$ CLCs for a 10,50 , and $250 \mathrm{GeV}$ particle interacting through an $n_{i}=$ standard operator. Comparisons are made to $\nu_{i}=$ standard, anapole, dipole, $q^{4}, q^{2}$, and $q^{-2}$ operators. The colors represent the value of $\widetilde{L}_{\text {min }} /$ d.o.f. As described above, cyan and lighter colors correspond to $95 \%$ or worse disagreement with the data. . . . . . . 273

A.2 $95 \%$ CLCs for a 10,50 , and $250 \mathrm{GeV}$ particle interacting through an $n_{i}=$ anapole moment operator. Comparisons are made to $\nu_{i}=$ standard, anapole, dipole, $q^{4}, q^{2}$, and $q^{-2}$ operators. The colors represent the value of $\widetilde{L}_{\min } /$ d.o.f. As described above, cyan and lighter colors correspond to $95 \%$ or worse disagreement with the data. 
A.3 95\% CLCs for a 10, 50, and $250 \mathrm{GeV}$ particle interacting through an $n_{i}=$ dipole moment operator. Comparisons are made to $\nu_{i}=$ standard, anapole, dipole, $q^{4}, q^{2}$, and $q^{-2}$ operators. The colors represent the value of $\widetilde{L}_{\text {min }} /$ d.o.f. As described above, cyan and lighter colors correspond to $95 \%$ or worse disagreement with the data.

A.4 95\% CLCs for a 10, 50, and $250 \mathrm{GeV}$ particle interacting through an $n_{i}=q^{2}$ operator. Comparisons are made to $\nu_{i}=$ standard, anapole, dipole, $q^{4}, q^{2}$, and $q^{-2}$ operators. The colors represent the value of $\widetilde{L}_{\min } /$ d.o.f. As described above, cyan and lighter colors correspond to $95 \%$ or worse disagreement with the data. . . . . . . . . . . . .

A.5 95\% CLCs for a 10, 50, and $250 \mathrm{GeV}$ particle interacting through an $n_{i}=q^{-4}$ operator. Comparisons are made to $\nu_{i}=$ standard, anapole, dipole, $q^{4}, q^{-2}$, and $q^{-4}$ operators. The colors represent the value of $\widetilde{L}_{\min } /$ d.o.f. As described above, cyan and lighter colors correspond to $95 \%$ or worse disagreement with the data. . . . . . . . . . . . . 277

D.1 Comparison of signal-only constraint (solid) and a $\chi^{2}$ goodness-of-fit test (dotted) for each experiment taking the sample spectrum from scalar DM decay to $e^{+} e^{-}$pairs that emit FSR. We show the limits derived from the data described in Sec. 6.2: HEAO-1 (orange), INTEGRAL (green), COMPTEL (blue), EGRET (red), and Fermi (yellow). . . . . . . . . . . . . . . . . . 288 


\section{LIST OF TABLES}

\section{$\underline{\text { Table }}$}

4.1 Physical properties of stable spin-odd nuclei present in detectors. The dominant argon nucleus has $A=40$, and there are no spin-odd isotopes with appreciable abundance. . . . . . . . . . .

$5.1 \quad$ Fits to the $3+1$ framework using 2010 and $2011 \bar{\nu}$ data. With the new MB data, the appearance and disappearance experiments disagree at more than the $4 \sigma$ level. . . . . . . . . . . . . . . . . . . . 122

5.2 Results of fits to the $3+1+1$ framework using 2010 and $2011 \mathrm{MB} \bar{\nu}$ data. With the new data, the appearance and disappearance data sets still disagree at about the $4 \sigma$ level, with only slight improvement over the $3+1$ case. . . . . . . . . . . . . . . . . . . . . . 125

6.1 Energy ranges, solid angles, and values of $J_{D}\left(J_{A}\right)$ for various DM density profiles. The NFW profile is taken from $[58,59]$, the Moore profile from [60], and the cored isothermal profile can be found in [61]. The profiles "Ein, $\alpha$ " are Einasto profiles [62] with slope parameter $\alpha .152$

8.1 Branching fractions for $h \rightarrow i i$ for $m_{h}=125.5 \mathrm{GeV}$ from [52]. . . . 239

8.2 Projected uncertainties on ratios of signal strength modifiers for the $14 \mathrm{TeV}$ LHC with 300 (3000) $\mathrm{fb}^{-1}$ of data. . . . . . . . . . . . . 241

B.1 Energies, mixings, and mass splitting sensitivities for each appearance experiment. ................... . 279

B.2 Energies, mixings, and mass splitting sensitivities for each disappearance experiment. . . . . . . . . . . . . . . . . 280

B.3 Fits to the reactor, gallium, and carbon anomalies. . . . . . . . . 282 


\section{LIST OF APPENDICES}

\section{Appendix}

A. Appendix: The Dark Matter Inverse Problem: Extracting Particle Physics from Scattering Events . . . . . . . . . . . . . . . . . . 271

B. Appendix: Neutrino Phenomenology in a $3+1+1$ Framework: Fit Details . . . . . . . . . . . . . . . . . . . . . . 278

C. Appendix: Neutrino Phenomenology in a $3+1+1$ Framework: Oscillation Formalism . . . . . . . . . . . . . . . . . . . . . . . . 283

D. Appendix: Constraining Light Dark Matter with Diffuse X-Ray and Gamma-Ray Observations . . . . . . . . . . . . . . . 286 


\section{CHAPTER I}

\section{Introduction}

I am writing this thesis during a time of great excitement within the field of particle physics, and I carry a new but well-founded optimism that exciting discoveries are around the corner. We are poised on the edge of great advances in two primary fields: we may soon understand the nature of the dark matter, and we can hope to fully characterize the Higgs field. These fields offer unprecedented windows into different facets of the natural universe, and they provide exciting opportunities to probe previously inaccessible physical effects. This thesis will focus on some advances in both of these areas.

\subsection{Dark Matter}

Understanding and constraining the dark matter has in many ways been my primary focus throughout my graduate career. Fritz Zwicky's first observations of the effects of dark matter are now eighty years old, and we have been waiting since that time to make more substantive contact with it on terrestrial scales. All of the extant support for the dark matter hypothesis is indirect, though quantitative: Zwicky's first arguments derived from charting the rotation curves of galaxies in the Coma cluster [1], and Vera Rubin collected convincing supporting evidence by focusing on stellar motion within the Andromeda galaxy [2] several decades later. 
Since that time, our understanding of these indirect arguments has deepened. We now understand the entire history of the assembly of the components of the visible universe as demanding the assistance of a large dark component. This dark matter affects the types of large-scale structures that we see around us, and, although the dark matter is believed to be a kind of particle, it has been convincingly proven not to have many of the ordinary properties that we take for granted in the particles around us. These arguments are given weight by direct observations of dark matter in environments where particle scattering is greatly enhanced, such as the observation of the Bullet Cluster [3]. Even more powerful support is given by comparing predictions of how the universe would look with and without dark matter, as quantified by the baryonic power spectrum [4]. Taken together, the circumstantial evidence for the existence of a dark type of matter is essentially overwhelming.

Still, our understanding of dark matter will remain unsatisfying until we are able to see its effects at local distances and on local timescales. The tests mentioned above all require the action of gravitational forces, which accumulate over long periods of time and over very large distance scales; on these scales, other forces cancel, and the details of the theory that fully describes dark matter is washed out. Although none of the arguments given above require it to be true, we hope that there are interactions of the dark matter besides those mediated by gravity. These interactions may simply couple the dark matter to itself in much the same way that electrons are repelled by other negatively charged electrons and attracted to positively charged atoms. The possibility of this kind of self-interacting dark matter is intriguing, but will not be considered in more depth in this thesis.

The more promising possibility is that dark matter interacts non-gravitationally with the standard model. For this to happen, the dark matter would need either to interact directly through the standard model force carriers like the $W$ or $Z$ bosons, the gluon, the higgs, or the photon, or to be joined by some kind of messenger particle 
that could transmit forces to the standard model. The former possibility is all but excluded by direct searches, so the second possibility is the only one that remains. We hope to discover these interactions in any of three search channels: we can detect the deposition of a dark matter particle's kinetic energy in direct detection experiments; produce and observe it as distinctive "missing energy" at high energy accelerators; or witness what is produced when two dark matter particles annihilate, known as "indirect detection" on local scales. Until this happens, we cannot be certain of many of the detailed properties of the dark matter particles, and thus we cannot know the underlying theory that ties dark matter to rest of the well-understood physical universe.

A precondition of being able to find the dark matter is knowing where to look. Thus, before we can realistically hope to discover dark matter, it is critical to consider all other indirect evidence in favor of and against otherwise acceptable dark matter properties. The a priori admissible parameter space is dauntingly large for exhaustive dedicated searches, so we must look to as wide a variety of physical systems as possible, both terrestrial and beyond, to see what they can already tell us about the dark matter. It is possible that a very generic subset of theoretically consistent dark matter properties are already effectively precluded by the existence or other behavior of certain extreme physical systems. By narrowing the window of the dark matter searches in this way (or by finding good reasons to focus their scope), we increase our chances of actually finding convincing evidence of dark matter.

Chapters II, III, IV, V, VI, and VII of this thesis will focus on some novel ideas for understanding and constraining the dark matter in a variety of ways. These chapters individually concentrate on specific, technical ideas for placing bounds on the ways that dark matter can behave. Taken together, I hope they convey a certain imaginative sense regarding the possible ways that dark matter may eventually be discovered. Here, I will briefly summarize each chapter: 
Chapter II: Turning Off the Lights: How Dark is Dark Matter?, with Hai-Bo Yu and Kathryn M. Zurek. Published in PRD [5].

In this paper, we considered the possibility that dark matter carries a small but non-zero electric charge. These types of models are generally referred to as "millicharged dark matter." The charge of the dark matter is given by the value $\epsilon$, which is the ratio of the dark matter charge to the electron charge, $\epsilon=q_{\mathrm{DM}} / e$. Aside from the mass of the dark matter, $\epsilon$ is the only free parameter, and together these suggest a well-defined parameter space to look for dark matter. In practice, $m_{\mathrm{DM}}$ and $\epsilon$ set the dark matter scattering and annihilation cross sections, and thus control all of the dark matter phenomenology.

The major qualitative difference between millicharged and regular dark matter is that, because millicharged dark matter scatters through the photon, the propagators scale like $1 / q^{2}$ and $1 / m_{\text {med }}^{2}$, respectively. Thus, millicharged dark matter is endowed with a large rate enhancement at low-momentum transfer. This could in principle have striking implications in the local galaxy, where the dark matter velocity is constrained by the size of the galactic gravitational potential. Locally, dark matter is highly nonrelativistic, and thus the low-momentum scattering dominates. In this way, small couplings could still mediate large event rates at direct detection experiments.

However, we found that astrophysics precludes this kind of dark matter scattering across the entire range of parameter space that may be probed by direct detection experiments. Specifically, supernova shock fronts efficiently remove the dark matter from the disk of the galaxy at the same time that the plane-parallel component of the galactic magnetic fields prevent the halo dark matter from being carried back in by its perpendicular velocity. Moreover, the parameters required for dark matter to be able to freeze out from annihilations in the early universe with the correct relic density are large enough that they are prohibited by ensuring that it has also decoupled from scattering off standard model particles at the time of recombination and structure 
formation.

For these reasons, the millicharged dark matter scenario is, in the absence of other theoretical structures, disfavored from being the only kind of dark matter particle.

Chapter III: Constraints on Scalar Asymmetric Dark Matter from Black Hole Formation in Neutron Stars, with Hai-Bo Yu and Kathryn M. Zurek. Published in PRD [6].

This study examined the effects the existence of a certain class of dark matter models could have on a certain class of stars. If enough dark matter mass could build up inside the star, it could collapse to form a black hole, which would in turn destroy the star. To illustrate how this hypothesis can give us insight into the nature of the dark matter, we chose a type of dark matter particle that has special properties which prevent it from annihilating when concentrated in large densities, and also prevent it from attaining a large pressure that could prevent gravitational collapse. Next, we selected a type of star with extreme gravitational features that would help to encourage dramatic responses from these types of particles.

The class of dark matter theories we studied is known as Asymmetric Dark Matter. The dark matter in this theory, like all of the ordinary matter around us, is not its own antiparticle. One especially appealing feature of these models is that the dark matter will accumulate and thermalize within a star rather than annihilating, so large quantities of the dark matter can build up over time. As this dark matter builds up, it can wreak havoc on the host star.

The stars we chose as standard targets are pulsars, which are rapidly rotating neutron stars that emit extremely regular pulses of electromagnetic radiation. All neutron stars have very deep potential wells and exceptionally high densities of standard model particles, so they serve as extreme but still representative examples of the way that dark matter could interact with matter on earth. Moreover, the spectra, the rate of pulsation, and the rate of change of this pulsation are well understood 
to indicate pulsar age and temperature. In this way, pulsar observations can give us detailed information about the nature of dark matter scattering in their environment.

For a given dark matter mass, we can calculate the critical quantity of Asymmetric Dark Matter that will be unstable against gravitational collapse. Then, given some details of the pulsar environment and the scattering cross section of dark matter off regular matter, we can calculate what mass of these particles will build up over the lifetime of the pulsar. The existence of old pulsars in regions speculated to contain large densities of dark matter therefore allows us to bound the scattering cross section of dark matter with regular matter.

For a broad range of dark matter masses, the bounds extracted from this procedure exceed the current and the projected scope of dark matter direct detection experiments by many orders of magnitude. Thus, these kinds of astrophysical constraints are a promising method of constraining new physics.

Chapter IV: The Dark Matter Inverse Problem: Extracting Particle Physics from Scattering Events, with Hai-Bo Yu and Kathryn M. Zurek. Published in $\mathrm{PRD}[7]$.

Most of my work focuses on the prospects of finding dark matter and learning as much as possible in the absence of explicit detection. In contrast, this paper considers the prospects for learning detailed information about the particle physics content of the dark matter interactions with the standard model under the assumption of observation in a variety of detectors. The resulting complementarity between the different detectors can remove some of the degeneracies in the dark matter model space and help select between competing theories of the dark sector.

We were specifically interested in the power of multiple direct detection experiments to discriminate between the momentum or velocity dependence of different operators. Aside from the standard dependence on the number of nucleons or on the total spin of the nucleus, dark matter may couple to the standard model in a way 
that scales with integer powers of momentum or velocity. Momentum dependence in the scattering cross section with the scaling $q^{n}, n=0, \pm 2, \pm 4$, or $v^{2}$ are simple to construct even at the basic level of dimension-six contact operators connecting the dark and the visible sectors. Thus, momentum dependence is a generic effect that we may need to empirically choose between. We were interested in the ability of different experiments to discriminate between these different hypotheses given only the Poisson-smeared energy spectra.

We found that the inclusion of experiments with different target nuclei is essential for the ability to extract this kind of information. The distinctive kinematics of the dark matter scattering in each target encodes enough information to allow the extraction of the momentum dependence in a robust way.

Chapter V: Neutrino Phenomenology in a 3+1+1 Framework, with Eric Kuflik and Kathryn M. Zurek. Published in PRD [8].

One specific theory of dark matter maintains that an extra species of neutrino with enough mass can be the dark matter. This new neutrino is called the sterile neutrino, and it interacts with the standard model via mass mixing with the conventional neutrinos. Aside from allowing interactions with the dark matter, this mixing is also capable in principle of explaining some anomalies observed in various neutrino experiments.

These anomalies refer to the high rate of appearance of neutrinos and antineutrinos of one flavor in beams which have started as neutrinos of a different flavor. This appearance phenomenon may be aided by the presence of a sterile neutrino. However, this sterile neutrino would similarly enhance the probability for particular flavors to disappear from other experiments, which seems not to occur at a high enough rate. Moreover, these rates seem to be considerably different between neutrinos and antineutrinos. 
Adding in a second neutrino at a mass scale far above the one probed by these experiments offers the possibility of reconciling this discrepancy. This idea, referred to as the $3+1+1$ neutrino model [9], relies on the additional $\mathrm{CP}$ violation to disentangle the neutrino predictions from the antineutrino ones, and also utilizes effective low-energy non-unitarity induced by the heavy state to decouple appearance and disappearance experiments.

Unfortunately, we discovered that even with the additional neutrino state, there is poor agreement in global fits to all available neutrino and antineutrino data. This makes the $3+1+1$ model unlikely to represent the final picture of the neutrino sector.

Chapter VI: Constraining Light Dark Matter with Diffuse X-Ray and Gamma-Ray Observations, with Rouven Essig, Eric Kuflik, Tomer Volansky, and Kathryn M. Zurek. Published in JHEP [10]. This work was completed during my term as a Fermilab Fellow in Theoretical Physics.

Cosmological photons provide a compelling route for dark matter searches. Photons are produced directly as the final state of many dark matter decays or annihilations, but they are also generically produced if dark matter decays or annihilates to charged particles. By choosing a specific mass range for the dark matter, one can ensure that the only final products are photons, neutrinos, and electrons and positrons, all of which are stable. Thus, the bounds from these mass ranges can be absolutely robust against any modeling uncertainty.

In this work, we considered very broad classes of dark matter models that would lead to dark matter candidates in this mass range, and studied the observable photon signals they would produce. To pick a particularly rich example, a massive sterile neutrino of the sort that would enter the $3+1+1$ framework has two decay modes: a two-body radiative decay, $\nu_{s} \rightarrow \nu \gamma$; and a three-body tree-level decay, $\nu_{s} \rightarrow \nu_{\ell} \ell^{+} \ell^{-}$. The first decay gives a monochromatic photon line while the second gives a spectrum of bremsstrahlung photons. Our results indicate that if the sterile neutrino is all of 
the dark matter, it is forced to have a lifetime exceeding the age of the universe by many orders of magnitude.

We considered several other classes of dark matter models and found bounds of similar strength over the mass range spanning from a few keV to a few hundred $\mathrm{MeV}$. Because we did not model the astrophysical backgrounds in any way, nor consider the propagation of the stable charged particles after productive, these bounds are more conservative than necessary, but also absolutely robust against any updates in the details of astrophysics.

Chapter VII: Dissecting the Gamma-Ray Background in Search of Dark Matter, with Ilias Cholis and Dan Hooper. Published in JCAP [11]. This work was undertaken and completed during my term as a Fermilab Fellow in Theoretical Physics.

As in the preceding paper, this work focuses on constraints derived from observations of cosmological photons. In contrast to that paper, however, the objective in this case was to extract aggressive constraints that represented more realistic bounds on the dark matter properties. We were specifically interested in the isotropic diffuse gamma ray background as a place to test these claims.

To do this, we built up an entire model of the background emission of various cosmological and astrophysical objects. For objects with well understood spectral features in other energy ranges, we correlated their brightness in those ranges with their brightness in gamma rays. We found that within the uncertainties of these fits, the astrophysical objects could quite plausibly account for all of the isotropic diffuse emission. Including dark matter in the fit then allowed us to place upper limits on the amount of acceptable annihilation in galaxies.

Dark matter annihilation proceeds in three distinct ways in this model. First, we required the use of statistical halo mass functions and subhalo boost factors up to the redshifts around the age of structure formation in order to understand the 
contribution of distant galaxies and galaxy clusters to the diffuse component. Next, we needed to add in a component of unresolved Milky Way subhaloes that would not appear as bright point sources in the galactic observations. Finally, the smooth part of the Milky Way halo would also give a large contribution to this emission. Depending on the assumptions that we made about the size and statistical properties of the substructure, both locally and cosmologically, any one of these three components could dominate the putative dark matter contribution.

We discovered no evidence for dark matter annihilation, but we forecasted that, under certain assumptions about the substructure functions, the bounds from this procedure would be among the strongest possible with a few more years of data taking.

\subsection{Higgs Physics}

In contrast to the null searches for dark matter, the particle physics community has recently been able to celebrate the positive results of a different search: namely, the discovery of the long-sought Higgs boson. This has prompted a burst of activity in both the theoretical and experimental communities, and also a surge of collaboration between the two. The search for the Higgs was long and tortuous, and its culmination closed a chapter in modern physics by completing the Standard Model. Now that the Standard Model has been finalized, we are for the first time in the position that the consistency of the best theory we have for terrestrial physics demands no additional particles.

The Standard Model is in some sense complete, but even if more detailed studies of the Higgs boson reveal no inconsistencies between the observations and the Standard Model predictions, the Standard Model will remain theoretically unfulfilling. This is because the Standard Model bears some worrisome theoretical baggage, centered on the Higgs boson, that we hope to understand more deeply. This theoretical 
discomfiture goes by the name of the "naturalness problem"

All fundamental particles bear a few inalienable properties. At a classical level, these are understood simply as the mass and the strength with which the particle interacts with other particles. At the deeper quantum level, these properties and others are encoded as charges or "quantum numbers." The Higgs is the first fundamental particle we have found with a quantum number of spin that is exactly zero. For this reason, the Higgs lacks some of the symmetry properties endowed to other particles that would allow us a deep understanding of precisely how it acquires mass. These symmetry properties indicate certain "natural" ranges for the mass parameters of the other particles, and the values we predict match up well with the values we observe. In the case of the Higgs, where this symmetry property is lacking, our principled guess for the Higgs mass is in gross violation of the observed value. We say that the Higgs mass is therefore unnatural. One of the most compelling questions in Higgs physics is why this guess is so far from the actual value.

The resolution to this problem may indicate the existence of new particles. Because the Higgs lacks the symmetries that ensure the natural values of other particle masses, a new symmetry may have profound implications for how the Higgs mass is set. This sensitivity to new symmetries means that we generally expect that its properties are quite sensitive to new kinds of physics. The lack of symmetries found in the Higgs boson also allow it to couple much more promiscuously than other particles, and for this reason our hopes are high for finding new physics in the Higgs sector in the form of entirely new particles. The Higgs serves as a kind of portal to new physics that we otherwise would be unable to probe. Hence, detailed studies of the Higgs offer substantial possibilities to reach far beyond our current theory and to learn about additional particles or new scales that could hint at the existence of physics beyond the Standard Model. For this reason, the task of understanding the Higgs in detail is now of paramount importance. 
Chapter VIII of this thesis deals with some new ideas regarding the structure of certain observable properties of the Higgs sector.

Chapter VIII: Inspecting the Higgs for New Weakly Interacting Particles, with Clifford Cheung and Kathryn M. Zurek. Published in JHEP [12].

This paper takes the viewpoint that there are two primary particle classes that will be discoverable primarily through Higgs precision data rather than direct searches. These new particles are uniquely able to alter the detailed properties of the Higgs. We expect to see effects that fall into two separate categories: tree-level rate suppression, induced by new neutral electroweak singlet particles; and loop-induced rate enhancement or suppression, mediated by new electroweak charged doublets. The interplay between these can deliver complex phenomenology which may be hinted at by the preliminary Higgs data.

Specifically, the early Higgs data confirmed the baseline expectation for production and decay rates due to the Higgs couplings to the $W$ and $Z$ vector bosons. Intriguingly, the data also pointed toward a non-negligible excess in the rate of decay through photons. The most naïve analysis indicates that it is difficult to decouple these two rates because the Higgs couplings to photons is dominated by a loop of virtual $W$ 's. However, the amount of freedom allowed in the model with both the tree-level and loop-induced modifications was enough to allow this decoupling at roughly the observed rate.

This phenomenology naturally fits into a popular framework that makes the Higgs mass natural and also furnishes some new particles beyond those in the Standard Model. We were able to demonstrate large enhancements to the photon rate paired with only minor deviations from the standard expectation for the $W$ and $Z$ rates. We were also able to provide a direct outline for matching underlying theory parameters with observational values. 


\subsection{Looking Forward}

Particle physics right now is so exciting because we appear to be on the threshold of some major advances. We have found the Higgs boson at long last, and we are seemingly starting to narrow our focus on the way that dark matter can interact with the Standard Model. An exploration of these subjects will form the bulk of this dissertation.

In addition to these advances, we have lately started to see a steady stream of new, positive, and unexpected results. Among other things, these results prove that we cannot predict ahead of time where the next exciting discovery will be made. In only the last few months, several unforeseen surprises have invigorated the particle physics community. These include the observation of an unexplained monochromatic X-ray photon line with the data of the Chandra and XMM-Newton spacecraft, [13] as well as the discovery of a large signature of tensor modes in the data of the BICEP2 telescope [14]. The new X-ray observations, if they are confirmed over time, would point to new physics in the dark sector, which might be richer and more varied than we have been expecting. The BICEP2 observations are commonly interpreted as a signal of a large energy density of gravitational waves in the early universe. This very large energy density implies that, in contrast to indications from recent experiments, the age of cosmological inflation may have taken place at extremely high energy scales. With additional observations, we may look forward to the possibility of advancing our understanding of cosmological inflation. These observations would represent our only plausible probe of physics at the extremely high scales that inflation now points to. Because of the fast-developing nature of the field, this thesis will not touch on these exciting topics, but these experiments or searches like them will undoubtedly shape the way we view physics in the next decade.

The most pressing issues in the field of particle physics right now are on the border

of observation and pure theory. Synthesizing the two requires being able to imagine all 
of the ways in which theories may be incarnated and all of the observations they can predict. It also requires the imagination of seeing how data can impact theories, and what sorts of constraints the theories must obey if they purport to be the explanation of the natural world.

Moving forward, we are in the midst of an immense opportunity to advance our understanding of the natural world. This will come primarily in the form of new developments in dark matter physics and increasingly precise measurements of the Higgs. I am excited about being poised on the edge of this exciting time. 


\section{Bibliography}

[1] F. Zwicky, Helv. Phys. Acta 6, 110 (1933).

[2] V. C. Rubin and W. K. Ford, Jr., Astrophys. J. 159, 379 (1970).

[3] D. Clowe, M. Bradac, A. H. Gonzalez, M. Markevitch, S. W. Randall, C. Jones and D. Zaritsky, Astrophys. J. 648, L109 (2006) [astro-ph/0608407].

[4] S. Dodelson, Int. J. Mod. Phys. D 20, 2749 (2011) [arXiv:1112.1320 [astroph.CO]].

[5] S. D. McDermott, H. -B. Yu and K. M. Zurek, Phys. Rev. D 83, 063509 (2011) [arXiv:1011.2907 [hep-ph]].

[6] S. D. McDermott, H. -B. Yu and K. M. Zurek, Phys. Rev. D 85, 023519 (2012) [arXiv:1103.5472 [hep-ph]].

[7] S. D. McDermott, H. -B. Yu and K. M. Zurek, Phys. Rev. D 85, 123507 (2012) [arXiv:1110.4281 [hep-ph]].

[8] E. Kuflik, S. D. McDermott and K. M. Zurek, Phys. Rev. D 86, 033015 (2012) [arXiv:1205.1791 [hep-ph]].

[9] A. E. Nelson, Phys. Rev. D 84, 053001 (2011) [arXiv:1010.3970 [hep-ph]].

[10] R. Essig, E. Kuflik, S. D. McDermott, T. Volansky and K. M. Zurek, JHEP 1311, 193 (2013) [arXiv:1309.4091 [hep-ph]].

[11] I. Cholis, D. Hooper and S. D. McDermott, JCAP 1402, 014 (2014) [arXiv:1312.0608 [astro-ph.CO]].

[12] C. Cheung, S. D. McDermott and K. M. Zurek, JHEP 1304, 074 (2013) [arXiv:1302.0314 [hep-ph]]. 
[13] E. Bulbul, M. Markevitch, A. Foster, R. K. Smith, M. Loewenstein and S. W. Randall, arXiv:1402.2301 [astro-ph.CO].

[14] P. A. R. Ade et al. [BICEP2 Collaboration], arXiv:1403.3985 [astro-ph.CO]. 


\section{CHAPTER II}

\section{Turning Off The Lights: How Dark Is Dark Matter?}

We consider current observational constraints on the electromagnetic charge of dark matter. The velocity dependence of the scattering cross-section through the photon gives rise to qualitatively different constraints than standard dark matter scattering through massive force carriers. In particular, recombination epoch observations of dark matter density perturbations require that $\epsilon$, the ratio of the dark matter to electronic charge, is less than $10^{-6}$ for $m_{X}=1 \mathrm{GeV}$, rising to $\epsilon<10^{-4}$ for $m_{X}=10 \mathrm{TeV}$. Though naively one would expect that dark matter carrying a charge well below this constraint could still give rise to large scattering in current direct detection experiments, we show that charged dark matter particles that could be detected with upcoming experiments are expected to be evacuated from the Galactic disk by the Galactic magnetic fields and supernova shock waves, and hence will not give rise to a signal. Thus dark

Written in collaboration with Hai-Bo Yu and K. M. Zurek. Originally published as Turning off the Lights: How Dark is Dark Matter?, Phys. Rev. D 83, 063509 (2011). arXiv:1011.2907 [hep-ph], MCTP-10-52. 
matter with a small charge is likely not a source of a signal in current or upcoming dark matter direct detection experiments.

\section{$2.1 \quad$ Introduction}

The nature of the dark matter (DM) remains a mystery. For DM in the $\mathrm{MeV}$ to $\mathrm{TeV}$ range, a wide variety of probes constrain the DM to be a Weakly Interacting Massive Particle (WIMP) which interacts with ordinary matter through suppressed couplings. These probes include direct detection of DM through nuclear recoils in underground detectors as well as indirect detection through DM annihilation to SM states in the sun (to neutrinos), in the Galactic center (to photons), and in the Galactic neighborhood (to charged particles). There are also significant constraints on DM couplings to ordinary matter through production and escape as missing energy at colliders. For a review, see [1].

Many of the most popular DM candidates naturally meet these stringent requirements. The neutralino from supersymmetry, for example, carries no electric charge and can interact only sub-weakly, via the Higgs or through small couplings to the $Z$ boson, evading the most stringent constraints from LEP, Tevatron, and direct detection experiments such as CDMS [2] and XENON10 [3]. Its thermal annihilation cross-section is below the bounds for indirect detection through neutrinos, photons, or charged cosmic rays. While WIMP DM has escaped direct and indirect detection thus far, it may be within reach. Direct detection experiments are scaling up, the reach of the LHC will begin to encompass weak scale DM candidates soon, and Fermi will continue to constrain DM annihilation in dwarf galaxies, the Galactic center, and in the halo.

At the same time, it is desirable to take as model-independent an approach as possible when constraining the nature of the DM. While in most popular models the DM carries no electromagnetic charge, periodically the notion of CHArged Massive 
Particle (a CHAMP) has reappeared in the literature [4-8]. In some of the earliest discussions of CHAMPs, the DM carried a full unit of charge, but it was realized that this runs into a wide range of very stringent constraints from searches for heavy hydrogen to direct detection in underground labs. Some of these constraints may not apply if the CHAMPs are expelled from the disk via shock waves from supernova remnants and screened from re-entry by the Galactic magnetic fields [9]. More recently, the possibility that DM carries a fractional or epsilon-charge has been considered and constrained via the CMB acoustic peaks [10]. Radio observations also constrain the electronic charge of the dark matter [11]. In addition, the notion that the DM carries a "dark charge" has recently been considered [12-17]. In these latter models the DM does not couple to the photon, but to a massless gauge force in the hidden sector.

In light of the current understanding of structure formation and cosmological history, we determine how large the DM charge can be while remaining consistent with current constraints. We also consider direct detection signals from epsilon-charged DM, and determine whether it is possible to give rise to the signals in DAMA [18] and CoGeNT [19] as discussed recently in [20]. Because we are answering a general question about the coupling of DM to the photon, we leave our discussion of models to a minimum. We note that the discussion encompassed by this paper does bring to light a number of constraints that strongly disfavor some recent models in the literature. We comment on these models below where relevant. DM may also have a magnetic or electric dipole; this has been thoroughly considered recently [21], and we do not discuss it here.

The outline of this paper is as follows. We begin with a brief discussion of models and the implications of this study for the viability of these models. We then review the relic density calculation before turning to constraints. We discuss halo shape constraints and the bound from scattering at recombination times. We discuss direct detection of charged particles in light of the signals from CoGeNT and DAMA, and the 
implications of the bounds discussed here for these experiments and models designed to fit them. Finally, we conclude.

\subsection{Models and General Considerations}

Since DM that carries an electric charge must conserve $U(1)_{\mathrm{EM}}$, it must be a Dirac particle. There are a number of models in the literature where the DM carries a fractional or epsilon-charge. If a dark photon is massive and kinetically mixes with the photon, an epsilon-charge arises in Stueckelberg models [22] on account of the unique form of Stueckelberg mass term. If, on the other hand, the dark photon is massless, kinetic mixing between the dark and visible photons induces an electric charge for the DM (or equivalently, a dark charge for visible states) [74]. This mechanism is utilized for example in the Mirror Charged DM model proposed by [20] to generate the signals in CoGeNT and DAMA. We will see that the constraints we discuss here strongly disfavor such a model as the explanation for these signals. In either case, we denote the charge of the DM as $\epsilon e$.

When determining the constraints on the DM charge, the essential features will be the irreducible coupling to the photon (and charged SM particles), and, more importantly, the velocity dependence of the scattering cross-section. For example, the Rutherford Scattering cross-section of DM off DM through a photon is

$$
\frac{\mathrm{d} \sigma_{X X}}{\mathrm{~d} \Omega_{*}}=\frac{\alpha_{\mathrm{em}}^{2} \epsilon^{4}}{m_{X}^{2} v_{\mathrm{rel}}^{4} \sin ^{4}\left(\theta_{*} / 2\right)},
$$

where $m_{X}$ is the DM mass, $v_{r e l}$ is the DM relative velocity, and $\theta_{*}$ is the scattering angle in the center-of-mass frame. Likewise, the scattering cross-section of DM off baryon is

$$
\frac{\mathrm{d} \sigma_{X b}}{\mathrm{~d} \Omega_{*}}=\frac{\alpha_{\mathrm{em}}^{2} \epsilon^{2}}{4 \mu_{b}^{2} v_{\mathrm{rel}}^{4} \sin ^{4}\left(\theta_{*} / 2\right)},
$$

where $\mu_{b}$ is the DM-baryon reduced mass. 
The important point phenomenologically is the very large enhancement in the scattering cross-section at low velocity, giving a hint for where to look for strong constraints on DM charge. Galactic constraints, where the DM has been heated through collapse and virialization, as we will see, tend to give weak constraints. In contrast, the tightest constraints come primordially, before collapse and heating occur, when the DM is highly non-relativisitic. In particular we will find that constraints on DM coupled to baryons at the time of recombination and DM coupled to baryons in protohalos can be very important, and this constraint will eliminate models whose charges are larger than about $10^{-6}$, dependent on the mass of the DM. This constraint eliminates a broad class of models.

On the other hand, this constraint does not eliminate DM with epsilon-charges that can give rise to a signal in direct detection experiments. We will find, however, that in the region where the DM could give rise to a signal in direct detection, one expects the DM to have been evacuated from the disk via supernova shock waves, and its re-entry to have been prevented by Galactic magnetic fields. Therefore, although direct detection experiments are extremely sensitive to small charges, we will find that charged DM, such as suggested in [20], could not plausibly give rise to a signal in a direct detection experiment. We now go through these constraints in detail.

\subsection{Relic Density Constraints}

We begin by discussing the constraints from the relic density. If the DM is nonthermally produced, its relic density depends on the production mechanism (for example, if the DM particle is produced via the decay of a mother particle, its relic density depends on the number density of the mother particle). In this scenario, constraints from the current relic abundance are highly model dependent. On the other hand, in the case of thermal relics, the DM density is simply determined by the thermally averaged annihilation cross section. As we will discuss explicitly, a charged 
DM consistent with all cosmological constraints must be non-thermally produced, unless it has additional interactions. We now review the relic density considerations.

The DM can annihilate to photon pairs and to charged fermion pairs through the photon. In general, the charged DM can also carry other SM or hidden sector quantum numbers and annihilate through these channels as well. In our analysis we will not specify these additional interactions in detail; instead, we maintain a less model-dependent view. We assume DM is in thermal equilibrium in the early universe and require the irreducible annihilation processes not overly deplete DM. By considering only the annihilation channels induced by the electromagnetic charge of the DM, we can derive upper bounds on the charge $\epsilon$ for a given mass $m_{X}$.

The annihilation cross sections of $X \bar{X} \rightarrow \gamma \gamma$ and $f \bar{f}$ at tree level are given by

$$
\left(\sigma_{\mathrm{an}} v_{\mathrm{rel}}\right)_{\gamma \gamma}=\frac{\pi \alpha_{\mathrm{em}}^{2} \epsilon^{4}}{m_{X}^{2}}
$$

and

$$
\left(\sigma_{\mathrm{an}} v_{\mathrm{rel}}\right)_{f \bar{f}}=\frac{\pi \alpha_{\mathrm{em}}^{2} \epsilon^{2}}{m_{X}^{2}} q_{f}^{2} N_{c} \sqrt{1-\frac{m_{f}^{2}}{m_{X}^{2}}}\left(1+\frac{m_{f}^{2}}{2 m_{X}^{2}}\right)
$$

respectively, where $q_{f}$ is the charge of the SM fermion in units of electron charge and $N_{c}$ is the color multiplicity of the fermion. The total annihilation cross section of the DM particle at tree level is $\left(\sigma_{\text {an }} v_{\text {rel }}\right)_{\text {tot }}=\left(\sigma_{\text {an }} v_{\text {rel }}\right)_{\gamma \gamma}+\sum_{f}\left(\sigma_{\text {an }} v_{\text {rel }}\right)_{f \bar{f}}$.

The tree level annihilation cross section is enhanced by the Sommerfeld effect in the low velocity dispersion [24-27]. DM freeze out with Sommerfeld enhancement has been discussed in $[28,29]$. Since the mediator of the Sommerfeld enhancement is the standard model photon with zero mass, this enhancement never saturates. The enhancement factor for the tree level S-wave annihilation cross section is given by

$$
S=\frac{\left(\alpha_{\mathrm{em}} \epsilon^{2} \pi\right) / v}{1-e^{-\left(\alpha_{\mathrm{em}} \epsilon^{2} \pi\right) / v}}
$$


where $v=v_{\text {rel }} / 2$ is the DM velocity in the center of mass frame. The thermally averaged total annihilation cross section including the Sommerfeld enhancement is given by

$$
\left\langle\sigma_{\text {an }} v_{\text {rel }}\right\rangle_{\text {tot }}=\left(\sigma_{\text {an }} v_{\text {rel }}\right)_{\text {tot }} \frac{x_{X}^{3 / 2}}{2 \sqrt{\pi}} \int_{0}^{\infty} S v_{\text {rel }}^{2} e^{-x_{X} v_{\text {rel }}^{2} / 4} d v_{\text {rel }}
$$

where we assume a Maxwell-Boltzmann distribution for DM particle and $x_{X} \equiv$ $m_{X} / T_{X}$ with $T_{X}$ as the DM temperature. The Sommerfeld enhanced annihilation tends to deplete DM particles with low velocity, which may distort the thermal distribution of the DM after kinetic decoupling. However, as we will show in the next section, the charged DM can couple to the thermal bath even during the recombination epoch, and therefore the Maxwell-Boltzmann distribution is a good approximation.

Following the standard procedure to calculate the abundance of a thermal relic [30, 31], freeze out occurs when

$$
\begin{aligned}
x_{f} & \approx \ln \xi-\frac{1}{2} \ln (\ln \xi) \\
\xi & =0.038 \kappa(2+\kappa) m_{\mathrm{pl}} m_{X}\left(g / \sqrt{g_{*}}\right)\left(\sigma_{\mathrm{an}} v_{\text {rel }}\right)_{\mathrm{tot}},
\end{aligned}
$$

where $x=m_{X} / T, T$ is the temperature of the thermal bath, and $g$ is the number of degrees of freedom of the DM particle; we take $g=4$, for a Dirac particle. The value of $\kappa$ is chosen to match the numerical solution; we set $\kappa=1$.

The present number density of the DM is the solution of the Boltzmann equation, which can be written as

$$
\frac{1}{Y\left(x_{\mathrm{s}}\right)}=\frac{1}{Y\left(x_{f}\right)}+\sqrt{\frac{\pi}{45}} m_{\mathrm{Pl}} m_{X} \int_{x_{f}}^{x_{s}} \frac{\left(g_{* s} / \sqrt{g_{*}}\right)\left\langle\sigma_{\mathrm{an}} v_{\mathrm{rel}}\right\rangle_{\mathrm{tot}}}{x^{2}} d x
$$

where $Y=n_{X} / s$ with $s$ the entropy density, and $x_{s}=T_{\mathrm{s}} / m_{X}$ with $T_{\mathrm{s}}=1 \mathrm{eV}$, where we stop the integration. Here we assume $X$ and $\bar{X}$ have the same number density, $n_{X}=n_{\bar{X}}$, and the total number density of the DM is their sum, $n_{X}+n_{\bar{X}}$. Before 


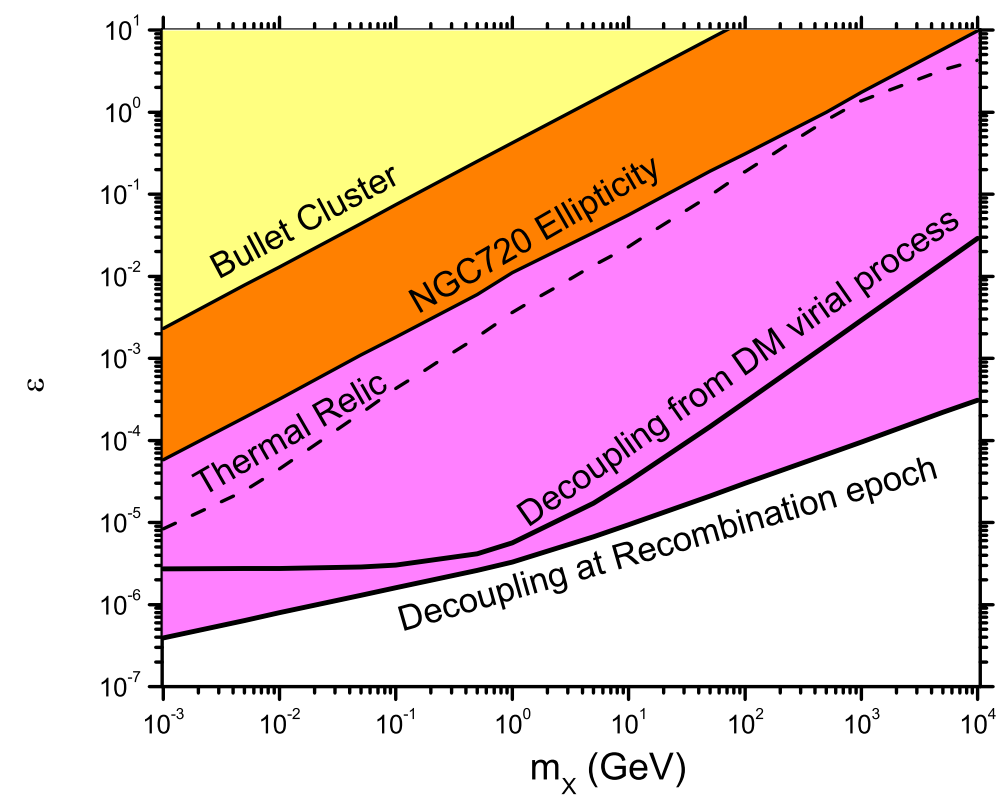

Figure 2.1: Constraints from various sources, from top to bottom: (i) Scattering in the bullet cluster and NGC720, (ii) DM as a charged thermal relic, and (iii) DM virial processes, and (iv) recombination epoch.

kinetic decoupling, the DM temperature is the same as the thermal bath temperature and drops as $a^{-1}$, where $a$ is the scale factor. After kinetic decoupling, the DM temperature drops as $a^{-2}$. Therefore, the enhancement factor scales as $S \sim x^{-1}$ and $S \sim x^{-2}$ with respect to $x$ before and after kinetic decoupling, respectively [28]. Since DM is tightly coupled to baryons through the massless photon until after the recombination epoch, $S \sim x^{-1}$ over the entire range of integration of Eq. (2.9). As we will show in Section IV, the elastic scattering rate rises as the temperature drops so that the DM may still be coupled to the thermal bath when the temperature is below $1 \mathrm{eV}$, even if $\epsilon$ is chosen to satisfy the relic density bound. 


\subsection{Structure Formation and CMB Constraints}

\subsubsection{Decoupling at the Recombination Epoch}

Charged DM particles interact with the Standard Model via a small coupling through the photon, so that Coulomb scattering can couple the charged DM to the baryon-photon plasma tightly even at low temperature. If the DM is still in kinetic equilibrium with the baryon-photon plasma during recombination, the DM density fluctuations can be washed out due to the radiation pressure and the photon diffusion (Silk damping [32]). The baryon acoustic peak structure will also be directly altered through the coupling. The effects of millicharged particles on CMB acoustic peaks have been discussed in Refs. $[10,33]$. It was found that if the millicharged particles couple to the baryon-photon plasma tightly during the recombination epoch they behave like baryons, and CMB observations put an upper limit on their abundance $\Omega_{\mathrm{mcp}} h^{2}<0.007$ (95\%) [10]. Here we assume that the DM is made of epsiloncharged particles and derive the relaxation time scale for the DM to reach kinetic equilibrium with baryons. To avoid damping effects on DM density fluctuations and CMB anisotropy constraints, we require that DM have completely decoupled from the photon-baryon plasma at the recombination epoch, and derive a bound on $\epsilon$ for a given DM mass.

We consider a DM particle that has momentum $p_{X}$ in its comoving frame. After each scattering event the magnitude of the DM momentum changes by an amount $\delta p_{X}$. The momentum transfer rate is thus given by

$$
\Gamma_{p}=\frac{\mathrm{d}\left\langle\delta p_{X}^{2}\right\rangle / \mathrm{dt}}{\left\langle p_{X}^{2}\right\rangle}
$$


The thermally averaged momentum transfer per unit time is

$$
\mathrm{d}\left\langle\delta p_{X}^{2}\right\rangle / \mathrm{dt}=\sum_{b=e, p} n_{b} \int d^{3} v_{B} d^{3} v_{X} f\left(v_{B}\right) f\left(v_{X}\right) d \Omega_{*} \frac{d \sigma_{X b}}{d \Omega_{*}} v_{\mathrm{rel}} \delta p_{X}^{2},
$$

where $d \sigma_{X b} / d \Omega_{*}$ is given by Eq. (2.2), $n_{b}$ is the number density of the baryon, and $\delta p_{X}^{2}$ is the momentum transfer after one collision:

$$
\delta p_{X}^{2}=2 \mu_{b}^{2} v_{\mathrm{rel}}^{2}\left(1-\cos \theta_{*}\right)
$$

Note that this quantity is reference frame independent. The thermally averaged momentum squared of the DM particle in its comoving frame is

$$
\left\langle p_{X}^{2}\right\rangle=\int d^{3} v_{X} f\left(v_{X}\right)\left(m_{X} v_{X}\right)^{2}=\frac{3}{2} m_{X}^{2} v_{0}^{2}=3 m_{X} T
$$

for a DM particle in a thermal Maxwell distribution. To evaluate the thermal average for $v_{\text {rel }}^{2}$, we derive a general formula. For a given function of $g\left(v_{\text {rel }}\right)$, we have

$$
\int d^{3} v_{a} d^{3} v_{b} f\left(v_{a}\right) f\left(v_{b}\right) g\left(v_{\text {rel }}\right)=\int d v_{\text {rel }} v_{\text {rel }}^{2} \frac{4}{\sqrt{\pi}} \frac{1}{\left(v_{0 a}^{2}+v_{0 b}^{2}\right)^{\frac{3}{2}}} e^{-\frac{v_{\text {rel }}^{2}}{v_{0 b}^{2}}+\frac{v_{v_{\text {el }}^{2}}^{2} v_{0 a}^{2}}{\left(v_{0 a}^{2}+v_{0 b}^{2} v_{0 b}^{2}\right.}} g\left(v_{\text {rel }}\right),
$$

where we assume $f\left(v_{a, b}\right)$ are Maxwellian distributions and $v_{0 a, b}$ are the most probable velocities for the $a$ and $b$ particles, respectively. By using this general formula, we have

$$
\mathrm{d}\left\langle\delta p_{X}^{2}\right\rangle / \mathrm{dt}=-\sum_{b=e, p} \frac{8 \sqrt{2 \pi} n_{b} \alpha_{\mathrm{em}}^{2} \epsilon^{2}}{\left(\frac{T}{m_{X}}+\frac{T}{m_{b}}\right)^{\frac{1}{2}}} \ln \left(\theta_{*}^{\min } / 2\right),
$$

where $\theta_{*}^{\min }$ is the cutoff in the $\Omega_{*}$ integral. Its value is set by the maximum impact parameter due to Debye screening effects in the plasma. This maximum impact 
parameter is related to the minimum scattering angle through

$$
b_{\max }=\frac{\alpha_{\mathrm{em}} \epsilon}{\left\langle\mu_{b} v_{\mathrm{rel}}^{2}\right\rangle} \cot \left(\theta_{*}^{\min } / 2\right)
$$

where $\left\langle\mu_{b} v_{\text {rel }}^{2}\right\rangle=3 T$ and $\cot \left(\theta^{\min _{*}} / 2\right) \simeq 2 / \theta_{*}^{\min }$ for small $\theta_{*}^{\text {min }}$. The impact parameter of the scattering must not be larger than the Debye screening length, so we have $b \leq b_{\max }=\lambda_{D}$. Thus

$$
\theta_{*}^{\min } \simeq \frac{2 \epsilon \alpha_{\mathrm{em}}}{3 T \lambda_{D}}
$$

where $\lambda_{D}=\sqrt{T /\left(4 \alpha_{\mathrm{em}} \pi n_{e}\right)}$ is the Debye length for the baryon plasma. Using Eq. (2.13), we then have a momentum transfer rate of:

$$
\Gamma_{p}=\sum_{B=e, p} \frac{8 \sqrt{2 \pi} n_{b} \alpha_{\mathrm{em}}^{2} \epsilon^{2} \mu_{b}^{\frac{1}{2}}}{3 m_{X} T^{\frac{3}{2}}} \ln \left[\frac{3 T \lambda_{D}}{\epsilon \alpha_{\mathrm{em}}}\right]
$$

If the DM is tightly coupled to the baryon-photon plasma during recombination, DM density fluctuations will be damped. CMB observations also place strong constraints on the total abundance of charged particles in the tightly-coupled regime. Here we require that the relaxation time of the momentum transfer rate is larger than the Hubble time at the recombination epoch; i.e.,

$$
\Gamma_{p}^{-1}\left(T_{R}\right)>t_{R}
$$

where $t_{R} \simeq 3.8 \times 10^{5}$ years [34], and $T_{R} \simeq 0.26 \mathrm{eV}$ is the temperature at the recombination epoch. We take the baryon number density at recombination $n_{e}=n_{p}=$ $\Omega_{b} \rho_{c} a_{R}^{-3} / m_{p}$, where $\Omega_{b} \simeq 0.023 h^{-2}, \rho_{c}=8.0992 h^{2} \times 10^{-47} \mathrm{GeV}^{4}, a_{R} \simeq 1 / 1091$, and $h \simeq 0.71$ [34]. This constraint is plotted in Figure 2.1. In the above analysis, we have implicitly assumed that photons couple to baryons efficiently during the recombination epoch despite the presence of charged DM. We checked that electron-photon 
Compton scattering can keep baryons and DM particles in the kinetic equilibrium with photons. This is because the DM density is not far from the baryon density. The same Compton drag force also suppresses the growth of the DM density perturbations.

Here we ignore the process of DM-photon Compton scattering. Since the cross section $\sigma_{X \gamma}=8 \pi \alpha_{\mathrm{em}}^{2} \epsilon^{4} /\left(m_{X}^{2}\right)$ is proportional to $\epsilon^{4}$ and the momentum transfer rate through this process is also highly suppressed kinematically at low temperature, we expect that the bound derived from the DM-photon decoupling is weak. As shown in Ref. [35], CMB anisotropies and matter power spectrum requires $\sigma_{X \gamma} / m_{X}<$ $10^{-32} \mathrm{~cm}^{2} \mathrm{GeV}^{-1}$. We can translate this limit to a bound on $\epsilon$ as $\epsilon<0.49\left(m_{X} /(1 \mathrm{GeV})\right)^{3 / 4}$, which is much weaker than the bound derived from the DM-baryon decoupling.

\subsubsection{Effect on the Dark Matter Virialization}

After recombination, radiation damping suppression is absent, but the efficient energy transfer between baryons and charged DM particles will modify the virialization process of the DM. Since baryons decouple from the thermal bath much later than DM particles, baryons are hotter than DM particles at redshift $z \sim 30$ when protohalos start to form. If there is a tight coupling between DM particles and baryons at this epoch, baryons will transfer energy to DM particles and heat them up. We can derive a bound on $\epsilon$ by requiring the energy transfer time be longer than the DM virialization time.

Eq. (2.18) is no longer valid for charged particles with slow motion in a neutral medium. At these late epochs, although it appears that the Born approximation condition $\epsilon^{2} \alpha_{\mathrm{em}} / v_{\text {rel }}<1$ may still be satisfied due to the smallness of $\epsilon$, the charged DM particle typically has a wavelength larger than the Bohr radius of the hydrogen atom, and one must take into account the screening effect. This effect is analogous to the energy loss of a slow-moving ion in the neutral medium, a result first derived 
by Lindhard and Scharff [36]. Lindhard's approximation is valid when the impact parameter is larger than the Bohr radius. When the protohalo forms at redshifts $z \sim 30$, the DM velocity dispersion is $\mathcal{O}\left(10^{-8} c\right)$. Its de Broglie wavelength is much larger than the Bohr radius under these conditions, and we expect that Lindhard's formula applies. We calculate the energy exchange of the charged DM in the hydrogen medium using Lindhard's formula,

$$
\frac{d E_{X}}{d \ell}=\frac{n_{H}}{m_{e}}\left[\frac{\pi^{2} \epsilon}{2.7183} \frac{m_{X}}{m_{X}+m_{H}}\right]
$$

where $n_{H}$ is the hydrogen number density, and we ignore the negligible effects of electron recoil [36] and other elements. The relaxation time scale is estimated as

$$
\tau_{X} \simeq\left\langle E_{X}\right\rangle\left\langle\frac{1}{v_{\mathrm{rel}}} \frac{d \ell}{d E_{X}}\right\rangle=\frac{3 \times 2.7183 m_{e}\left(m_{H}+m_{X}\right)}{4 \sqrt{2} \pi^{3 / 2} n_{H} \epsilon} \sqrt{\frac{T_{H}}{m_{H}}+\frac{T_{X}}{m_{X}}}
$$

where $E_{X}=m_{X} v_{\text {rel }}^{2} / 2$. We take $\sqrt{T_{H} / m_{H}} \sim 10^{-6} c$, and $\sqrt{T_{X} / m_{X}} \sim 10^{-8} c$ at $z \sim 30$

In the usual cold DM scenario, DM collapses and virializes at a redshift of $z \sim 30$. In over-dense regions the density is about 178 times larger than the average density at the same epoch [37], and the violent relaxation time scale is

$$
\tau_{\mathrm{vir}} \sim\left(G \rho_{\mathrm{tot}}\right)^{-1 / 2}
$$

where $\rho_{\text {tot }}=\rho_{X}+\rho_{\bar{X}}$ and $\rho_{\text {tot }} \sim 178 \bar{\rho}_{\text {tot }}=178 \Omega_{X} \rho_{c}(1+z)^{3}$. Now we demand $\tau_{X}>\tau_{\text {vir }}$ and obtain an upper bound on $\epsilon$,

$$
\epsilon<2.9 \times 10^{-6}\left(\frac{m_{H}+m_{X}}{1 \mathrm{GeV}}\right)
$$

which is shown in Fig. 1. 


\subsection{Dark Matter Halo Constraints}

The strongest constraints on the coupling of DM to the photon come from scattering considerations rather than annihilations because of the large scattering crosssection enhancement at low velocities. In the previous section, we explored the effects at high redshift from observations of universe at recombination temperatures. The constraints are particularly strong in this regime. However, lower redshift observations can also be used to test the charged DM hypothesis.

\subsubsection{Elliptical galaxies}

If the DM self-interaction through Coulomb scattering is strong enough to create an $\mathcal{O}(1)$ change in the momentum of DM particles within the age of galaxies, it will isotropize the velocity dispersion and lead to more spherical halos. The collisions also cause heat conduction from the hot outer parts to the cooler inner parts of DM halos, giving rise to the formation of a core with a shallow density profile. These expectations have been confirmed by simulations in the hard sphere scattering limit [38-43]. In addition, observations of elliptical DM halos in clusters constrain self-interactions [44], while observations of elliptical DM halos in galaxies provide the strongest constraints on self-interacting DM models $[14,43,46]$. In this paper, we will follow the analysis of Ref. $[14,43,46]$ and use the ellipticity of NGC 720 to derive the upper bound of the electric charge of the DM.

To estimate how the ellipticity of NGC 720 may be used to constrain the charge of the DM, we calculate the relaxation time due to momentum transfer. We then assume the relaxation time scale is the same as the time scale for isotropizing the mass distribution of the DM halo. By using Eq. (2.1), the thermally-averaged momentum transfer rate inside the halo can be evaluated from as

$$
\Gamma_{e}=-16 \pi \alpha_{\mathrm{em}}^{2} \epsilon^{4} \frac{\rho_{\mathrm{tot}}}{3 m_{X}^{3} v_{0}^{2}} \int d v_{\mathrm{rel}} v_{\mathrm{rel}}^{2} \sqrt{\frac{2}{\pi}} \frac{1}{v_{0}^{3}} e^{-\frac{v_{\mathrm{rel}}^{2}}{2 v_{0}^{2}}} \frac{1}{v_{\mathrm{rel}}} \ln \left(\theta_{*}^{\min } / 2\right)
$$


where $\rho_{\text {tot }}=\rho_{\mathrm{X}}+\rho_{\overline{\mathrm{X}}}$ is the total DM density of the halo, and the minimal scattering angle $\theta_{*}^{\text {min }}$ is given by $\theta_{*}^{\min } \simeq 4 \alpha_{\mathrm{em}} \epsilon /\left(3 m_{X} v_{0}^{2} \lambda_{D}\right)$ with $\lambda_{D}=m_{X}^{2} v_{0}^{2} /\left(8 \pi \epsilon^{2} \alpha_{\mathrm{em}} \rho_{\text {tot }}\right)$ as the Debye screening length of the DM halo.

The elliptical galaxy NGC 720 is well-studied [47,48]. In Ref. [48], X-ray isophotes were used to extract the ellipticity of the underlying matter distribution, and the DM halo of NGC 720 was found to be elliptical at $5 \mathrm{kpc}$ and larger radii. At $5 \mathrm{kpc}$, the

DM density is $\rho_{\text {tot }}=4 \mathrm{GeV} / \mathrm{cm}^{3}$, and the radial velocity dispersion $\overline{v_{r}^{2}}=v_{0}^{2}(r) / 2 \simeq$ $(240 \mathrm{~km} / \mathrm{s})^{2}$ [43]. To derive the constraints on $\epsilon$ for the given $m_{X}$ from the observed halo shapes, we require

$$
\Gamma_{e}^{-1}>10^{10} \text { years. }
$$

That is, the average time scale to create $\mathcal{O}(1)$ change in the DM particle momentum must be greater than the galaxy's lifetime. This bound, weaker than the constraint derived from decoupling at the time of recombination, is depicted in Fig. 2.1.

\subsubsection{The Bullet Cluster}

In the Bullet Cluster system, a subcluster has collided with and moved through a larger cluster. These clusters have three major components that each behave very differently during the collision: stars, gas, and DM. The visible stars pass through without colliding, but the highly collisional X-ray gas slows down significantly. Gravitational lensing shows that the DM tracks the stars, which are effectively collisionless. These observations have been used to place stringent bounds on the self-interaction of the DM [49]. These bounds are derived through different considerations including the offset between the gas and DM, the high velocity of the subcluster, and the survival of the subcluster after the collision. It turns out that the survival of the subcluster puts the strongest bound on the self-interaction of DM [49]. 
We follow the approach of Ref. [49] to derive bounds on the DM charge. The analysis of Ref. [49] is based on a hard sphere scattering cross section, but we relax this assumption. For Rutherford scattering, the subcluster experiences a net loss of DM particles if particles in both the main cluster and the subcluster have velocities larger than the escape velocity of the subcluster. We define the scattering angle $\theta$ to be measured in the rest frame of the subcluster, which implies $\theta=\theta_{*} / 2$, where $\theta_{*}$ is the scattering angle in the frame of the center mass of two colliding particles.

The particle loss condition detailed above can be satisfied if $\sin \theta$ is in the following range:

$$
\frac{v_{\mathrm{esc}}}{v_{1}}<\sin \theta<\sqrt{1-\frac{v_{\mathrm{esc}}^{2}}{v_{1}^{2}}},
$$

where $v_{1} \sim 4800 \mathrm{~km} / \mathrm{s}$ is the velocity of the main cluster incoming particles before the collision and $v_{\text {esc }} \sim 1200 \mathrm{~km} / \mathrm{s}$ is the escape velocity of the subcluster. We

assume that the subcluster sees the main cluster with a surface number density $\Sigma_{m} \sim$ $0.3 \mathrm{~g} / \mathrm{cm}^{3}$ [49], and demand that the particle loss fraction $f$ be smaller than $30 \%$, i.e.

$$
f=\frac{\sum_{m}}{m_{X}} \int d \Omega_{*} \frac{d \sigma_{X X}}{d \Omega_{*}}=\frac{\sum_{m}}{m_{X}} \frac{4 \pi \alpha_{\mathrm{em}}^{2} \epsilon^{4}}{m_{X}^{2} v_{1}^{2}}\left(\frac{1}{v_{\mathrm{esc}}^{2}}-\frac{1}{v_{1}^{2}-v_{\mathrm{esc}}^{2}}\right)<30 \% .
$$

The Bullet Cluster bound is given in Fig. 2.1.

\subsection{Direct Detection of Charged Dark Matter}

Because of the large enhancement of the scattering cross-section at low velocity, even DM with a very small charge can give rise to a large scattering cross-section in direct detection experiments. In [20], it was found, for example, that a charge of $\epsilon \sim 10^{-9}$ was sufficient to give rise to the relatively large signals in CoGeNT and DAMA. Thus, if correct, direct detection experiments have a potential to give rise to even tighter constraints on epsilon-charged DM with mass in the range $m_{X} \sim$ 


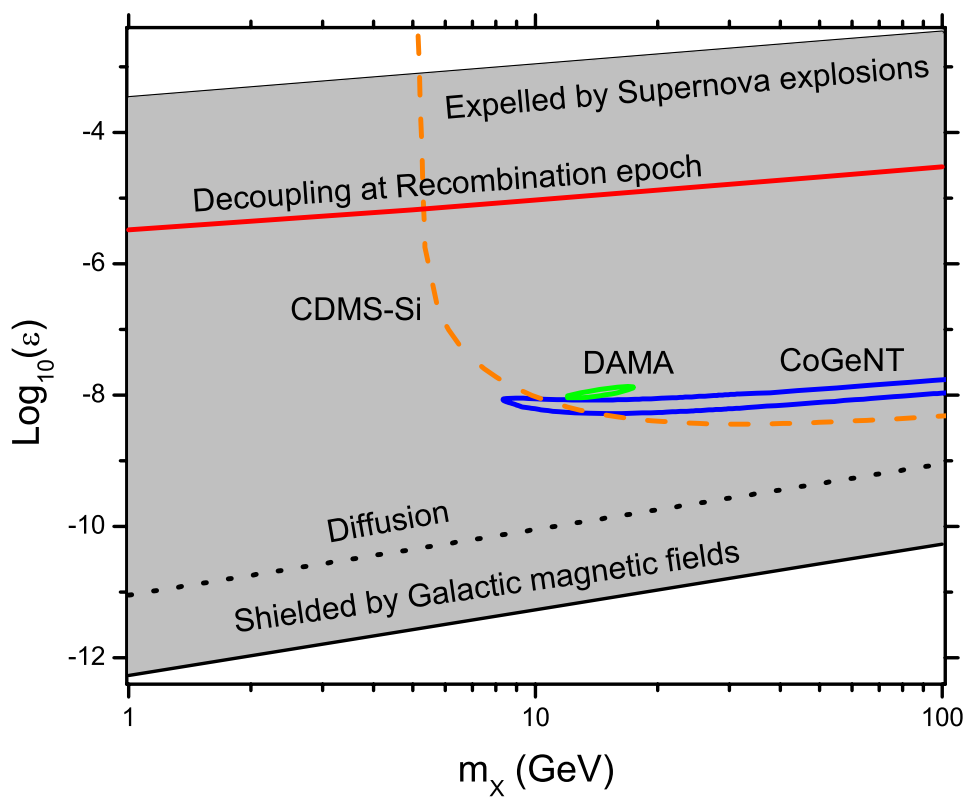

Figure 2.2: CoGeNT (blue), DAMA (green) allowed regions at 99\% C.L. The CDMS$\mathrm{Si}$ (yellow) line is included as a sample exclusion at 99\% C.L. In the gray area, the charged DM is evacuated from the Galactic disk. Also shown the bound from Recombination epoch (red). Below the dotted line (black), charged DM may diffuse to the disk. 
$10 \mathrm{GeV}-1 \mathrm{TeV}$. We find, however, that in the range of charges where DM could give rise to a signal in a direct detection experiment the DM will necessarily have been efficiently evaporated from the Disk, and thus one expects no signal. We begin by a review of the signal in direct detection experiments.

\subsubsection{Direct Detection Basics}

The rate for scattering is

$$
\frac{d R}{d E_{R}}=N_{T} \frac{\rho_{\chi}}{m_{\chi}} \int_{|\vec{v}|>v_{m i n}} d^{3} v v f\left(\vec{v}, \vec{v}_{e}\right) \frac{d \sigma}{d E_{R}}
$$

where $v_{\text {min }}=\frac{\sqrt{2 m_{N} E_{R}}}{2 \mu_{N}}$, and $\mu_{N}$ is the reduced mass of the nucleus-DM system. We take the velocity distribution $f\left(\vec{v}, \vec{v}_{e}\right)$ to be a modified Boltzmann distribution

$$
f\left(\vec{v}, \vec{v}_{e}\right) \propto\left(e^{-\left(\vec{v}+\vec{v}_{e}\right)^{2} / v_{0}^{2}}-e^{-v_{e s c}^{2} / v_{0}^{2}}\right) \Theta\left(v_{e s c}^{2}-\left(\vec{v}+\vec{v}_{e}\right)^{2}\right)
$$

where explicit expressions for the velocity integrals from this distribution can be found in [50]. The additional term is to allow for a smooth cut-off of the velocity distribution near the Galactic escape velocity $v_{e s c}$. The Earth's speed relative to the Galactic halo is $v_{e}=v_{\odot}+v_{\text {orb }} \cos \gamma \cos \left[\omega\left(t-t_{0}\right)\right]$ with $v_{\odot}=v_{0}+12 \mathrm{~km} / \mathrm{s}, v_{\text {orb }}=30 \mathrm{~km} / \mathrm{s}, \cos \gamma=$ $0.51, t_{0}=$ June 2 nd and $\omega=2 \pi /$ year. We take as a standard case $v_{0}=220 \mathrm{~km} / \mathrm{s}$, and we fix $v_{e s c}=500 \mathrm{~km} / \mathrm{s}$ and the local DM density $0.3 \mathrm{GeV} / \mathrm{cm}^{3}$.

A standard calculation relates the differential rate for scattering off nuclei to the scattering rate off a nucleus $\sigma_{N}$,

$$
\frac{d \sigma}{d E_{R}}=\frac{m_{N} \sigma_{N}}{2 \mu_{N}^{2} v^{2}}
$$

For the standard spin-independent case, this rate is related to a scattering off protons, 
$\sigma_{p}$, through

$$
\sigma_{N}=\sigma_{p} \frac{\mu_{N}^{2}}{\mu_{n}^{2}} \frac{\left[f_{p} Z+f_{n}(A-Z)\right]^{2}}{f_{p}^{2}} F^{2}\left(E_{R}\right),
$$

where $\mu_{n}$ is the DM-nucleon reduced mass and $f_{p}$ and $f_{n}$ are the DM couplings to the neutron and proton. We set $f_{n}=0$ since the coupling is assumed to be through the photon. We make use of a Helm form factor $F\left(E_{R}\right)=\frac{3 j_{1}\left(q r_{0}\right)}{\left(q r_{0}\right)} e^{-(q s)^{2} \mathrm{fm}^{2} / 2}$, where $r_{0}=\left(\left(1.2 A^{1 / 3}\right)^{2}-5 s^{2}\right)^{1 / 2} \mathrm{fm}$, with $s=1$. The scattering cross-section off nuclei through the photon is

$$
\sigma_{N}=\frac{16 \pi \alpha^{2} \epsilon^{2} Z^{2} \mu_{N}^{2}}{q^{4}}
$$

which is to be inserted in Eq. (2.28) to obtain the total rate as a function of energy. As an example of the typical DM charge $\epsilon$ that can be probed with direct detection experiments, we show the constraints one obtains from the CDMS, DAMA and CoGeNT experiments in Fig. (2.2). One can see that the viable region is well below the structure formation constraints labeled in the figure. Note that in Ref. [20], the mirror DM velocity dispersion depends on the particle mass, and is typically smaller than the rotation speed $v_{0}=220 \mathrm{~km} / \mathrm{s}$, so the allowed regions for both DAMA and CoGeNT as well as the excluded region for CDMS shift to larger DM mass (larger than $20 \mathrm{GeV}$ ) compared to the fitting presented in Fig. 2.2. Since the shift is more significant for the light target nuclei, the mirror DM model features a DAMA region which is not excluded by CDMS, as depicted in [20]. We next discuss how DM with charges in this range will have been evacuated from the disk at the present day, eliminating any possible signal in a direct detection experiment.

\subsubsection{Evacuation of Charged DM from the Disk}

The charged DM interacts with the magnetic fields of the Galaxy in addition to baryons in the disk. Since the large-scale magnetic field in the Milky Way is mostly parallel to the plane of the Galactic disk, the charged DM particle in the halo may 
not be able to penetrate the disk if its gyroradius is smaller than the height of the disk. This magnetic shielding effect for the millicharged particle has been discussed in the Ref. [9]. The gyroradius is given by

$$
R_{\mathrm{g}} \simeq 5.4 \times 10^{-11} \mathrm{pc}\left(\frac{m_{X}}{1 \mathrm{GeV}}\right)\left(\frac{1}{\epsilon}\right)\left(\frac{v_{X}}{270 \mathrm{~km} / \mathrm{s}}\right)\left(\frac{5 \mu \mathrm{G}}{B}\right)<H_{\mathrm{d}}
$$

where $H_{\mathrm{d}} \sim 100 \mathrm{pc}$ is the typical height of the Galactic disk. So the Galactic magnetic field prevents charged DM from entering the disk if $\epsilon>5.4 \times 10^{-13}\left(m_{X} / \mathrm{GeV}\right)$.

It is possible that some quantity of charged DM can remain in the disk from the time when the disk formed. Subsequently, however, shock waves generated by supernova (SN) explosions can blow these particles out of the disk if the acceleration time scale $\left(\tau_{\text {acc }} \simeq 10^{7}\right.$ years $)$ is shorter than the cooling time scale [9]. The cooling time scale due to the scattering with electrons is given by

$$
\tau_{\text {cool }}=\frac{m_{X} m_{e} v_{X}^{3}}{8 \pi \alpha_{\mathrm{em}}^{2} \epsilon^{2} n_{e}}\left[\ln \left(\frac{\mu_{e} v_{X}^{2} \lambda_{D}}{\alpha_{\mathrm{em}} \epsilon}\right)\right]^{-1}
$$

where we take $n_{e} \sim 0.025 / \mathrm{cm}^{3}$, the Coulomb logarithm $\ln \left(\mu_{e} v_{X}^{2} \lambda_{D} / \alpha_{\mathrm{em}} \epsilon\right) \sim 30$ for the parameter range of interest. By demanding $\tau_{\text {cool }}<\tau_{\text {acc }}$, we get $\epsilon<3.4 \times$ $10^{-4} \sqrt{m_{X} / \mathrm{GeV}}$. Here we assume the epsilon-charged DM is efficiently accelerated by the Fermi mechanism. This is true when the gyroradius of the charged DM is smaller than the length of shock waves. Since the length of the shock wave can be $\sim 100$ pc [51], as long as $\epsilon>5.4 \times 10^{-13}\left(m_{X} / \mathrm{GeV}\right)$, the charged DM will be accelerated along with baryons over a time scale $\tau_{\text {acc }} \simeq 10^{7}$ years.

Hence, if the DM charge is in the range $5.4 \times 10^{-13}\left(m_{X} / \mathrm{GeV}\right)<\epsilon<3.4 \times$ $10^{-4} \sqrt{m_{X} / \mathrm{GeV}}$, the number density of the DM is negligible in the disk. We note that this constraint strongly disfavors the charged DM explanations of DAMA and CoGeNT experiments, because the experimentally preferred value is $\epsilon \sim 10^{-9}$ and $m_{X} \sim \mathcal{O}(10-20) \mathrm{GeV}$. One possible way to relax this constraint is to consider the 
diffusion of DM into the disk. The Galactic magnetic field is not perfectly parallel, and in fact has a large nonperturbative turbulent component. The charged DM particles may diffuse into the disk as they interact with the turbulent magnetic field. The diffusion time scale can be estimated as $\tau_{\text {diff }} \sim 5 H_{\mathrm{d}}^{2} /\left(3 R_{\mathrm{g}} v_{X}\right)$ [52]. If the $\tau_{\text {diff }}$ is smaller than the acceleration time scale $\tau_{\text {acc }}$, the charged DM may be able to diffuse to the vicinity of the earth and leave a signal in direct detection experiments. This signal is sensitive to the DM number density, which highly depends on the diffusion process and will in general be smaller than $0.3 \mathrm{GeV} / \mathrm{cm}^{3}$. We find that the charge has to satisfy $\epsilon<9 \times 10^{-12}\left(m_{X} / \mathrm{GeV}\right)$ with $v_{X}=270 \mathrm{~km} / \mathrm{s}$ and $B=5 \mu \mathrm{G}$, which is too small to fit DAMA and CoGent data.

\subsection{Conclusions}

We have discussed the cosmological and direct detection constraints on charged DM. We considered in particular relic density, halo shape, large scale structure, recombination-era coupling, and direct detection constraints. We found that charged DM must have additional annihilation modes or be non-thermally produced if it is to satisfy the CMB constraints on DM couplings to baryons, which require the DM charge be smaller than $\epsilon \sim 10^{-6}$ for $1 \mathrm{GeV} \mathrm{DM}$ (weakening to $\epsilon \sim 10^{-4}$ at $10 \mathrm{TeV}$ ). We discussed the possibility that one or more of these constraints is nullified by supernova shock waves blowing charged DM out of the disk, and showed as a result that DM with epsilon charge $10^{-9}$ cannot be an explanation for the CoGeNT or DAMA excesses, though the DM-baryon interaction cross-section is large enough. In addition, no signal in direct detection experiments could be expected in future experiments, as DM with large enough charge to generate a sizable DM-nucleus interaction cross-section would have been evacuated from the disk.

While the idea of charged DM is in many ways an elegant one, its feasibility as a DM candidate, it appears, is strongly constrained. Further, these tight constraints 
also apply to any model where a massless dark photon kinetically mixes with the visible photon, so that fields charged under $U(1)_{\mathrm{EM}}$ pick up a dark charge. This study presents constraints on a wide variety of hidden sector models that may be useful in the continued hunt for DM.

\section{Acknowledgments}

We thank Matt Buckley, Jonathan Feng, Manoj Kaplinghat, Aaron Pierce, Dan Feldman, and Itay Yavin for discussions. 


\section{Bibliography}

[1] G. Jungman, M. Kamionkowski, K. Griest, Phys. Rept. 267, 195-373 (1996). [hep-ph/9506380].

G. Bertone, D. Hooper, J. Silk, Phys. Rept. 405, 279-390 (2005). [hep$\mathrm{ph} / 0404175]$.

J. L. Feng, [arXiv:1003.0904 [astro-ph.CO]].

[2] Z. Ahmed et al. [The CDMS-II Collaboration], Science 327, 1619 (2010) [arXiv:0912.3592 [astro-ph.CO]].

[3] E. Aprile et al. [XENON100 Collaboration], Phys. Rev. Lett. 105, 131302 (2010) [arXiv:1005.0380 [astro-ph.CO]].

J. Angle et al. [XENON10 Collaboration], Phys. Rev. D 80, 115005 (2009) [arXiv:0910.3698 [astro-ph.CO]].

[4] H. Goldberg and L. J. Hall, Phys. Lett. B 174, 151 (1986).

[5] A. De Rujula, S. L. Glashow, U. Sarid, Nucl. Phys. B333, 173 (1990)

[6] S. Dimopoulos, D. Eichler, R. Esmailzadeh and G. D. Starkman, Phys. Rev. D 41, 2388 (1990).

[7] S. Davidson and M. E. Peskin, Phys. Rev. D 49, 2114 (1994) [arXiv:hep$\mathrm{ph} / 9310288]$.

[8] S. Davidson, S. Hannestad and G. Raffelt, JHEP 0005, 003 (2000) [arXiv:hepph/0001179].

[9] L. Chuzhoy and E. W. Kolb, JCAP 0907, 014 (2009) [arXiv:0809.0436 [astro$\mathrm{ph}]$. 
[10] S. L. Dubovsky, D. S. Gorbunov and G. I. Rubtsov, JETP Lett. 79, 1 (2004) [Pisma Zh. Eksp. Teor. Fiz. 79, 3 (2004)] [arXiv:hep-ph/0311189].

[11] S. Gardner and D. C. Latimer, Phys. Rev. D 82, 063506 (2010) [arXiv:0904.1612 [hep-ph]].

[12] J. L. Feng, H. Tu, H. -B. Yu, JCAP 0810, 043 (2008). [arXiv:0808.2318 [hepph]].

[13] L. Ackerman, M. R. Buckley, S. M. Carroll et al., Phys. Rev. D79, 023519 (2009). [arXiv:0810.5126 [hep-ph]].

[14] J. L. Feng, M. Kaplinghat, H. Tu and H. B. Yu, JCAP 0907, 004 (2009) [arXiv:0905.3039 [hep-ph]].

[15] A. Ibarra, A. Ringwald, C. Weniger, JCAP 0901, 003 (2009). [arXiv:0809.3196 [hep-ph]].

[16] N. Kaloper, A. Padilla, JCAP 0910, 023 (2009). [arXiv:0904.2394 [astroph.CO]].

[17] D. C. Dai, K. Freese and D. Stojkovic, JCAP 0906, 023 (2009) [arXiv:0904.3331 [hep-ph]].

[18] R. Bernabei et al. [DAMA Collaboration], Eur. Phys. J. C 56, 333 (2008) [arXiv:0804.2741 [astro-ph]].

[19] C. E. Aalseth et al. [ CoGeNT Collaboration ], [arXiv:1002.4703 [astro-ph.CO]].

[20] R. Foot, arXiv:1008.0685 [hep-ph]. R. Foot, Phys. Rev. D78, 043529 (2008). [arXiv:0804.4518 [hep-ph]].

[21] K. Sigurdson, M. Doran, A. Kurylov et al., Phys. Rev. D70, 083501 (2004). [astro-ph/0406355]. 
[22] D. Feldman, Z. Liu, P. Nath, Phys. Rev. D75, 115001 (2007). [hep-ph/0702123 [HEP-PH]]. D. Feldman, Z. Liu, P. Nath and G. Peim, Phys. Rev. D 81, 095017 (2010) [arXiv:1004.0649 [hep-ph]].

[23] B. Holdom, Phys. Lett. B 166, 196 (1986).

[24] A. Sommerfeld, Annalen der Physik 403, 257 (1931).

[25] H. Baer, K. m. Cheung and J. F. Gunion, Phys. Rev. D 59, 075002 (1999) [arXiv:hep-ph/9806361].

[26] J. Hisano, S. Matsumoto and M. M. Nojiri, Phys. Rev. D 67 (2003) 075014 [arXiv:hep-ph/0212022]. J. Hisano, S. Matsumoto and M. M. Nojiri, Phys. Rev. Lett. 92, 031303 (2004) [arXiv:hep-ph/0307216]. J. Hisano, S. Matsumoto, M. M. Nojiri and O. Saito, Phys. Rev. D 71, 063528 (2005) [arXiv:hep$\mathrm{ph} / 0412403]$.

[27] M. Cirelli, A. Strumia and M. Tamburini, Nucl. Phys. B 787, 152 (2007) [arXiv:0706.4071 [hep-ph]]. J. March-Russell, S. M. West, D. Cumberbatch and D. Hooper, JHEP 0807, 058 (2008) [arXiv:0801.3440 [hep-ph]]. M. Cirelli, M. Kadastik, M. Raidal and A. Strumia, Nucl. Phys. B 813, 1 (2009) [arXiv:0809.2409 [hep-ph]]. N. Arkani-Hamed, D. P. Finkbeiner, T. R. Slatyer and N. Weiner, Phys. Rev. D 79, 015014 (2009) [arXiv:0810.0713 [hep-ph]]. M. Pospelov, A. Ritz, Phys. Lett. B671, 391-397 (2009) [arXiv:0810.1502 [hepph]]. P. J. Fox and E. Poppitz, Phys. Rev. D 79, 083528 (2009) [arXiv:0811.0399 [hep-ph]]. M. Lattanzi, J. I. Silk, Phys. Rev. D79, 083523 (2009). [arXiv:0812.0360 [astro-ph]]. R. Iengo, JHEP 0905, 024 (2009) [arXiv:0902.0688 [hep-ph]]. R. Iengo, arXiv:0903.0317 [hep-ph]. S. Cassel, arXiv:0903.5307 [hep-ph]. T. R. Slatyer, JCAP 1002, 028 (2010) [arXiv:0910.5713 [hep-ph]]. L. Visinelli, P. Gondolo, [arXiv:1007.2903 [hep-ph]]. 
[28] J. L. Feng, M. Kaplinghat and H. B. Yu, arXiv:1005.4678 [hep-ph]. J. B. Dent, S. Dutta and R. J. Scherrer, Phys. Lett. B 687, 275 (2010) [arXiv:0909.4128 [astro-ph.CO]]. J. Zavala, M. Vogelsberger and S. D. M. White, Phys. Rev. D 81, 083502 (2010) [arXiv:0910.5221 [astro-ph.CO]].

[29] J. Hisano, S. Matsumoto, M. Nagai, O. Saito and M. Senami, Phys. Lett. B 646, 34 (2007) [arXiv:hep-ph/0610249]. Q. Yuan, X. J. Bi, J. Liu, P. F. Yin, J. Zhang and S. H. Zhu, JCAP 0912, 011 (2009) [arXiv:0905.2736 [astro-ph.HE]]. H. Iminniyaz and M. Kakizaki, arXiv:1008.2905 [astro-ph.CO]. S. Mohanty, S. Rao and D. P. Roy, arXiv:1009.5058 [hep-ph]. S. Hannestad and T. Tram, arXiv:1008.1511 [astro-ph.CO]. A. Hryczuk, R. Iengo, P. Ullio, [arXiv:1010.2172 [hep-ph]].

[30] P. Gondolo and G. Gelmini, Nucl. Phys. B 360, 145 (1991).

[31] E. W. Kolb and M. S. Turner, Front. Phys. 69, 1 (1990).

[32] J. Silk, Astrophys. J. 151, 459 (1968).

[33] C. Burrage, J. Jaeckel, J. Redondo et al., JCAP 0911, 002 (2009). [arXiv:0909.0649 [astro-ph.CO]].

[34] http://lambda.gsfc.nasa.gov/product/map/current/parameters.cfm

[35] C. Boehm, A. Riazuelo, S. H. Hansen and R. Schaeffer, Phys. Rev. D 66, 083505 (2002) [arXiv:astro-ph/0112522]. C. Boehm, P. Fayet and R. Schaeffer, Phys. Lett. B 518, 8 (2001) [arXiv:astro-ph/0012504]. C. Boehm and R. Schaeffer, arXiv:astro-ph/0410591.

[36] J. Lindhard and M. Scharff, Phys. Rev. 124, 128 (1961).

[37] M. Kamionkowski and S. Profumo, Phys. Rev. Lett. 101, 261301 (2008) [arXiv:0810.3233 [astro-ph]]. 
[38] R. Dave, D. N. Spergel, P. J. Steinhardt and B. D. Wandelt, Astrophys. J. 547, 574 (2001) [arXiv:astro-ph/0006218].

[39] N. Yoshida, V. Springel, S. D. M. White and G. Tormen, Astrophys. J. 535, L103 (2000) [arXiv:astro-ph/0002362].

[40] B. Moore, S. Gelato, A. Jenkins, F. R. Pearce and V. Quilis, Astrophys. J. 535, L21 (2000) [arXiv:astro-ph/0002308].

[41] M. W. Craig and M. Davis, arXiv:astro-ph/0106542.

[42] C. S. Kochanek and M. J. White, Astrophys. J. 543, 514 (2000) [arXiv:astro$\mathrm{ph} / 0003483]$.

[43] D. N. Spergel and P. J. Steinhardt, Phys. Rev. Lett. 84, 3760 (2000) [arXiv:astro-ph/9909386].

[44] J. Miralda-Escude, arXiv:astro-ph/0002050.

[45] J. L. Feng, M. Kaplinghat and H. B. Yu, Phys. Rev. Lett. 104, 151301 (2010) [arXiv:0911.0422 [hep-ph]].

[46] M. Ibe and H. B. Yu, Phys. Lett. B 692, 70 (2010) [arXiv:0912.5425 [hep-ph]].

[47] D. A. Buote, T. E. Jeltema, C. R. Canizares and G. P. Garmire, Astrophys. J. 577, 183 (2002) [arXiv:astro-ph/0205469].

[48] P. J. Humphrey, D. A. Buote, F. Gastaldello, L. Zappacosta, J. S. Bullock, F. Brighenti and W. G. Mathews, Astrophys. J. 646, 899 (2006) [arXiv:astro$\mathrm{ph} / 0601301]$.

[49] M. Markevitch, A. H. Gonzalez, D. Clowe et al., Astrophys. J. 606, 819-824 (2004). [astro-ph/0309303].

[50] A. L. Fitzpatrick, K. M. Zurek, [arXiv:1007.5325 [hep-ph]]. 
[51] R. D. Blandford, J. P. Ostriker, Astrophys. J. 221, L29-L32 (1978).

[52] F. J. Sanchez-Salcedo, E. Martinez-Gomez, [arXiv:0812.0797 [astro-ph]]. F. J. Sanchez-Salcedo, E. Martinez-Gomez, J. Magana, JCAP 1002, 031 (2010). [arXiv:1002.3145 [astro-ph.CO]]. 


\section{CHAPTER III}

\section{Constraints on Scalar Asymmetric Dark Matter from Black Hole Formation in Neutron Stars}

We consider possibly observable effects of Asymmetric Dark Matter (ADM) in neutron stars. Since dark matter does not self-annihilate in the ADM scenario, dark matter accumulates in neutron stars, eventually reaching the Chandrasekhar limit and forming a black hole. We focus on the case of scalar ADM, where the constraints from Bose-Einstein condensation and subsequent black hole formation are most severe due to the absence of Fermi degeneracy pressure. We also note that in some portions of this constrained parameter space, non-trivial effects from Hawking radiation can modify our limits. We find that for scalar ADM with mass between 5 $\mathrm{MeV}$ and $13 \mathrm{GeV}$, the constraint from nearby neutron stars on the scattering cross section with neutrons ranges from $\sigma_{n} \lesssim 10^{-45} \mathrm{~cm}^{2}$ to $10^{-47} \mathrm{~cm}^{2}$.

Written in collaboration with Hai-Bo Yu and K. M. Zurek. Originally published as Constraints on Scalar Asymmetric Dark Matter from Black Hole Formation in Neutron Stars, Phys. Rev. D 85, 023519 (2012). arXiv:1103.5472 [hep-ph], MCTP-11-16. 


\subsection{Introduction}

The characteristics of dark matter (DM) and the nature of its production mechanism have so far eluded description. While cosmological observations provide compelling evidence for its existence, its mass and the nature of its interactions with the standard model remain unknown. One popular hypothesis holds that the DM interacts with standard model particles via the weak interaction and is also selfannihilating. In this weakly interacting massive particle (WIMP) scenario [1], the correct relic density of DM is a natural consequence of the thermal history of the early Universe.

Alternatively, DM may carry a conserved charge, analogous to baryon number. This Asymmetric DM (ADM) scenario is motivated by the fact that the DM and baryon densities are of the same order of magnitude. The earliest models attempting to relate the DM to the baryon asymmetry made use of electroweak sphalerons [2] or out-of-equilibrium decay [3]. The former often run into tight constraints from LEP measurements. In contrast, the modern incarnation of ADM makes use of higher dimension operators to transfer the asymmetry in a robust way that is relatively free of electroweak constraints [4]. ADM models prefer to have DM mass around a few $\mathrm{GeV}$ (see for example $[5,7,77]$ ), which is consistent with hints from recent direct detection experiments $[5,8]$. It is also possible for ADM to have weak scale mass $[10,11]$, or mass well below a $\mathrm{GeV}[8]$.

The DM mass and its scattering cross section with nuclei have been constrained by various underground direct detection experiments $[3,14,15]$, as well as by particle colliders [16-19]. In this article, we study the properties of ADM through its impacts on stellar systems. It has long been appreciated that a finite DM-nucleon cross section would result in DM capture in stars [20-22]. In the WIMP scenario, DM annihilation can generate an additional heat source, which may affect stellar formation [23] and evolution [24], or cause anomalous heating of white dwarfs [25, 26] 
and neutron stars [27-29]. In the ADM case, DM particles do not annihilate and hence provide no additional power for stars. However, since there is no annihilation to deplete ADM particles, stars can accumulate far more ADM particles than usual WIMPs, which can lead to different effects. For example, it could have implications for solar physics [30-32], or change the mass-radius relation of neutron stars [33-35]. The most extreme possibility is that captured particles can become self-gravitating, forming a black hole that will eventually destroy the host stars [36-38].

Recently, constraints on fermionic ADM through the survival of compact stars have been discussed in $[29,39]$. In a certain class of ADM models, the DM candidate is a boson $[7,77]$. In this paper, we study constraints on this scalar ADM from compact stars. Scalar DM particles differ from fermions by spin statistics, which has a significant impact on black hole formation conditions. Black hole formation occurs only when the total number of self-gravitating DM particles is larger than the Chandrasekhar limit [40]. Fermions obey the Pauli exclusion principle, and the Chandrasekhar limit is set by the balance between gravity and the Fermi pressure, while scalar particles have no Fermi pressure to hinder gravity. In this case, the lower limit for gravitational collapse is determined by the balance between gravity and the pressure induced by the zero point energy, which is much smaller than the Pauli pressure experienced by fermions. Therefore, we derive much stronger constraints on scalar ADM from compact stars. Since neutron stars have much higher matter density and escape velocity than any other stars, we will mainly focus on neutron stars. Note that neutron star constraints on scalar DM has been discussed in [36]. In this early work, the cross section for the DM capture is set by the geometric cross section, and the DM mass range is between $1 \mathrm{GeV}$ and $100 \mathrm{TeV}$. In this work, we treat the DM-neutron cross section as a free parameter and use neutron stars to constrain it. Other important considerations that we treat here are the effect of Bose-Einstein condensation, which significantly alters the constraint derived on the DM-neutron 
scattering cross-section, and Hawking radiation, which modifies our constraints for high mass DM. We also explore a wider DM mass range and use the most recent neutron star data.

This paper is organized as follows. In Sec. II, we discuss the Chandrasekhar limit for fermions and bosons. In Sec. III, we discuss ADM capture in neutron stars. In Sec. IV, we discuss thermalization, condensation and black hole formation of captured scalar ADM in neutron stars. In Sec. V, we discuss Hawking radiation and destruction of the host neutron star. In Sec. VI, we discuss observational constraints. We present our conclusions in Sec. VII.

\subsection{Chandrasekhar Limit}

First, we review the derivation of the Chandrasekhar limit for a system of fermions. Suppose there are $N$ fermions of mass $m$ distributed in a sphere with radius $R$, so that the number density of fermions is approximately $N / R^{3}$. Due to the Pauli exclusion principle, the average distance between two fermions is $\sim R / N^{1 / 3}$. The uncertainty principle requires that each fermion have Fermi momentum $p \sim N^{1 / 3} / R$. If the total number $N$ is small and $m>p \sim N^{1 / 3} / R$, the system is in the non-relativistic limit. The average energy per fermion is

$$
E \sim-\frac{G N m^{2}}{R}+\frac{1}{m}\left(\frac{N^{1 / 3}}{R}\right)^{2}
$$

where $G$ is Newton's constant. Once the gravitational and Fermi pressures reach equilibrium, the system can have a stable spherical configuration with radius

$$
R \sim \frac{1}{G m^{3} N^{1 / 3}}
$$


As $N$ increases, the radius shrinks and the Fermi momentum increases; eventually fermions become relativistic with total energy

$$
E \sim-\frac{G N m^{2}}{R}+\frac{N^{1 / 3}}{R}
$$

If the total number of the fermions increases beyond the limit

$$
N_{\text {Cha }}^{\text {fermion }} \sim\left(\frac{1}{G m^{2}}\right)^{3 / 2}=\left(\frac{M_{p l}}{m}\right)^{3} \simeq 1.8 \times 10^{51}\left(\frac{100 \mathrm{GeV}}{m}\right)^{3}
$$

where $M_{p l}=1.2211 \times 10^{19} \mathrm{GeV}$ is the Planck scale, the gravitational energy will dominate the total particle energy and gravitational collapse will occur. This is the famous Chandrasekhar limit [40].

Now we discuss bosons. Similar to the fermion case, the gravitational collapse occurs when particles are relativistic. But the bosonic system is significantly different from the fermionic system because it has no Fermi pressure to hinder gravity. Since the bosons are confined inside a sphere with radius $R$, they have zero point energy $1 / R$ due to the uncertainty principle in the relativistic limit. Therefore, the typical energy for a boson in a sphere of radius $R$ is

$$
E \sim-\frac{G N m^{2}}{R}+\frac{1}{R}
$$

Again, the radius cancels in the critical limit. In this case, the Chandrasekhar limit is

$$
N_{C h a}^{b o s o n} \simeq\left(\frac{M_{p l}}{m}\right)^{2} \simeq 1.5 \times 10^{34}\left(\frac{100 \mathrm{GeV}}{m}\right)^{2}
$$

Comparing Eq. (3.4) and Eq. (3.6), we can see that for a given particle mass, a particle that obeys Bose-Einstein statistics will experience gravitational collapse much more readily than a particle that obeys Fermi-Dirac statistics.

When the total number of DM particles accumulated in a neutron star surpasses 
the Chandrasekhar limit, the captured DM particles collapse to a black hole and destroy the host neutron star. Therefore, observations of old neutron stars can be used to constrain the DM-neutron scattering cross section. Since bosons have much smaller Chandrasekhar limit than fermions, we can obtain stronger limits on bosonic DM. In this work, we take typical neutron star parameters $M_{n}=1.44 M_{\odot}, R_{n}=10.6 \mathrm{~km}$ and the central density $\rho_{B}=1.4 \times 10^{15} \mathrm{~g} / \mathrm{cm}^{3}[29,41]$.

\subsection{Capture of Asymmetric Dark Matter in Neutron Stars}

The accretion of DM onto stars has been studied in [20-22]. In this section, we review the basic formulae for the capture of asymmetric DM in neutron stars.

In the absence of annihilation, the number of DM particles in a star is determined by the differential equation

$$
\frac{d N_{X}}{d t}=C_{B}
$$

where $N_{X}$ is the total number of DM particles in the star and $C_{B}$ is the DM capture rate through scattering with baryons. In this study, we assume there is no symmetric component and neglect DM annihilation completely. In some ADM models, a portion of the symmetric component can be regenerated by oscillation effects $[8,11]$. For those models, bounds derived from the survival of the neutron stars can be weaker.

Additionally, we ignore the self-capture effect. Dark matter may have a sizable self-interaction that leads to self-capture [42]. However, the self-capture saturates when the sum of the individual self-scattering cross sections becomes larger than the geometrical area over which the DM particles thermally distribute. As we will show in the next section, due to the large baryon density the captured DM particles are thermally distributed in a very small region of radius $\sim 1 \mathrm{~m}$ in the core of the neutron star. We have checked that the baryonic capture always dominates the DM accretion process in neutron stars for the parameter space of interest, even if we take the upper 
limit of the self-scattering cross section allowed by the elliptical halo shape bound [43].

\subsubsection{Capture rate}

The number of DM particles in the neutron star is set by $C_{B}$. Since neutrons are degenerate in the neutron stars, capture can occur only when the momentum transfer is larger than the difference between the Fermi momentum and the neutron momentum. As we will show, this will affect the capture efficiency significantly for DM with mass less than $1 \mathrm{GeV}$. For larger DM mass the effect is negligible, because the momentum transfer is always sizable.

The accretion rate $C_{B}$ is given by [21]

$$
C_{B}=4 \pi \int_{0}^{R_{n}} r^{2} \frac{d C_{B}(r)}{d V} d r
$$

where $R_{n}$ is the radius of the neutron star and the capture rate per unit volume for an observer at rest with respect to the DM distribution is given by

$$
\frac{d C_{B}(r)}{d V}=\sqrt{\frac{6}{\pi}} n_{X}(r) n_{B}(r) \xi \frac{v(r)^{2}}{\bar{v}^{2}}\left(\bar{v} \sigma_{X B}\right)\left[1-\frac{1-\exp \left(-B^{2}\right)}{B^{2}}\right] .
$$

Here $n_{X}(r)$ is the ambient DM number density; $n_{B}(r)$ is the number density of the stellar baryons; $\bar{v}$ is the DM velocity dispersion around the neutron star; $v(r)$ is the escape velocity of the neutron star at the given radius $r ; \sigma_{X B}$ is the effective scattering cross section between DM particles and nucleons in the neutron star; and $\xi$ takes into account the neutron degeneracy effect on the capture. The factor $B^{2}$ is given by

$$
B^{2}=\frac{3}{2} \frac{v(r)^{2}}{\bar{v}^{2}} \frac{\mu}{\mu_{-}^{2}}
$$

where $\mu=m_{X} / m_{B}$ and $\mu_{-}=(\mu-1) / 2$.

Now we specify the factor $\xi$. All energy levels below the Fermi momentum $p_{F}$ 
have been occupied. During the scattering process, if the momentum transfer to the neutron is larger than $p_{F}$, the scattered neutron can be excited above the Fermi surface. In this case, all neutrons can participate the capture process, and the capture efficiency is $\xi=1$. On the other hand, if the momentum transfer $\delta p$ is less than $p_{F}$, only those neutrons with momentum larger than $\sim p_{F}-\delta p$ can participate in the capture process. The fraction of these neutrons is $\sim \delta p / p_{F}$, so we can approximate $\xi$ as $\xi \simeq \delta p / p_{F}$. Depending upon the momentum transfer $\delta p$, we can parameterize $\xi$ as

$$
\xi=\operatorname{Min}\left[\frac{\delta p}{p_{F}}, 1\right]
$$

When the DM particle approaches the surface of the neutron star, its velocity is close to the escape velocity. Hence, the typical momentum transfer is $\delta p \simeq \sqrt{2} m_{r} v_{e s c}$, where $m_{r}=m_{X} m_{B} /\left(m_{X}+m_{B}\right)$ is the reduced mass, and typically $v_{e s c} \simeq 1.8 \times 10^{5} \mathrm{~km} / \mathrm{s}$. The Fermi momentum is $p_{F} \simeq\left(3 \pi^{2} \rho_{B} / m_{B}\right)^{1 / 3} \simeq 0.575 \mathrm{GeV}$ for $\rho_{B}=1.4 \times 10^{15} \mathrm{~g} / \mathrm{cm}^{3}$. Therefore, $\xi \simeq 1$ for all $m_{X} \gtrsim 1 \mathrm{GeV}$. In contrast, the capture rate is suppressed by a factor $\sim m_{X} v_{e s c} / p_{F}$ if the DM mass smaller than the neutron mass.

To estimate the capture rate, we take the conservative limit that $v(r)=v\left(R_{n}\right) \equiv$ $v_{e s c}$, and we assume that $n_{X}(r)$ and $n_{B}(r)$ are independent of the radius; thus, the total capture rate can be simplified to

$$
C_{B} \simeq \sqrt{\frac{6}{\pi}} \frac{\rho_{X}}{m_{X}} \frac{v_{e s c}^{2}}{\bar{v}^{2}}\left(\bar{v} \sigma_{X B}\right) \xi N_{B}\left[1-\frac{1-\exp \left(-B^{2}\right)}{B^{2}}\right]
$$

where $N_{B}$ is the total number of neutrons in the host star. When $B^{2} \gg 1$, the term in the square bracket is close to one; for typical values $v_{\text {esc }} \simeq 1.8 \times 10^{5} \mathrm{~km} / \mathrm{s}$ and $\bar{v} \simeq 220 \mathrm{~km} / \mathrm{s}$, and a DM mass smaller than $9.4 \times 10^{5} \mathrm{GeV}$, this condition is obtained. If DM has mass larger than $\sim 9.4 \times 10^{5} \mathrm{GeV}$, a lower probability to lose enough kinetic energy to be captured after single scatter results [29]. In our numerical work, we use the full expression of Eq. (4.1). 


\subsubsection{Total Number of ADM in Neutron Stars}

Here we only consider DM particles captured by the neutron star itself and neglect those the neutron star can inherit from its progenitor. Compared to the neutron star phase, the progenitor usually has much lower density and shorter lifetime which results in lower capture efficiency. The total number of DM particles captured by the neutron star is given by the solution of Eq. (3.7)

$$
N_{X}=C_{B} t
$$

To evaluate $C_{B}$, we note that if the sum of individual nucleon-DM scattering cross sections is larger than geometric surface area of the star, the capture rate will saturate. Therefore, the capture rate increases with the cumulative nucleon-DM scattering cross section $\sigma_{t o t}=N_{B} \sigma_{n}$, where $\sigma_{n}$ is the DM-neutron elastic scattering cross section, as long as $\sigma_{\text {tot }}$ is smaller than $\sigma_{\text {geom }}=\pi R_{n}^{2}$; that is, we can constrain the individual scattering cross-section $\sigma_{n}$ as long as $\sigma_{n}$ is less than or equal to $\sigma_{\max }=\pi R_{n}^{2} / N_{B}$. Taking typical neutron star parameters $M_{n}=1.44 M_{\odot}$ and $R_{n}=10.6 \mathrm{~km}$, we estimate the maximum cross section as [29]

$$
\sigma_{\max }=2.1 \times 10^{-45} \mathrm{~cm}^{2}\left(\frac{R_{n}}{10.6 \mathrm{~km}}\right)^{2}\left(\frac{1.44 M_{\odot}}{M_{n}}\right)
$$

and the effective cross section is given by

$$
\sigma_{X B}=\operatorname{Min}\left[\sigma_{n}, \sigma_{\max }\right]
$$

Note that since we consider scattering off only one nucleon, this scattering can be regarded as either spin-dependent (SD) or spin-independent (SI).

Now we can estimate the total number of ADM in the neutron star at a given time, using generic parameters $v_{\text {esc }}=1.8 \times 10^{5} \mathrm{~km} / \mathrm{s}, \bar{v}=220 \mathrm{~km} / \mathrm{s}$, and $N_{B} \simeq 1.7 \times 10^{57}$. 
In the regime $m_{X} \gtrsim 1 \mathrm{GeV}$, we have $\xi \simeq 1$, which gives

$$
N_{X} \simeq 2.3 \times 10^{44}\left(\frac{100 \mathrm{GeV}}{m_{X}}\right)\left(\frac{\rho_{X}}{10^{3} \mathrm{GeV} / \mathrm{cm}^{3}}\right)\left(\frac{\sigma_{X B}}{2.1 \times 10^{-45} \mathrm{~cm}^{2}}\right)\left(\frac{t}{10^{10} \text { years }}\right)
$$

When the DM mass is less than $\sim 1 \mathrm{GeV}$, the degeneracy effect is important on the capture process so that $\xi \simeq \sqrt{2} m_{X} v_{e s c} / p_{F}$, and we have

$$
N_{X} \simeq 3.4 \times 10^{46}\left(\frac{\rho_{X}}{10^{3} \mathrm{GeV} / \mathrm{cm}^{3}}\right)\left(\frac{\sigma_{X B}}{2.1 \times 10^{-45} \mathrm{~cm}^{2}}\right)\left(\frac{t}{10^{10} \text { years }}\right) .
$$

It is interesting to note that the DM number does not depend on the DM mass in the second case.

In the above derivation of $N_{X}$, we have assumed that the evaporation effect is negligible for the DM. Now we estimate the DM mass scale below which the evaporation is relevant. Since energy states below the Fermi surface are occupied, only those neutrons with momentum above $p_{F}$ can transfer kinetic energy to the DM. Since $T \ll p_{F}$ for the neutron star, the number of these free neutrons is order $\sim 10^{-8}$ smaller than that of the neutrons in the Fermi sea. So the scattering probability for the DM evaporation is highly suppressed. Furthermore, compared to the sun, neutron stars have much higher density and deeper gravitational wells, so it is much more difficult to accelerate trapped DM above the escape velocity through interactions with neutrons. To evaporate from the neutron star, the DM has to gain enough energy such that its velocity is larger than the escape velocity of the neutron star. Because of the degeneracy effect, the typical energy transfer from the free neutron is $\sim T$; so the evaporation effect is relevant only when the DM mass is less than $\sim 2 T / v_{\text {esc }}^{2} \sim 48 \mathrm{eV}$ for $T=10^{5} \mathrm{~K}$, which is much below the lower mass limit of our constraints $\sim 2 \mathrm{keV}$ in the most optimistic case. Therefore, we can safely ignore the evaporation process. 


\subsection{Asymmetric Scalar Dark Matter in Neutron Stars}

\subsubsection{Thermalization}

When DM particles are captured by the neutron star they lose energy via scattering with neutrons, and soon attain thermal equilibrium with the star. To estimate the thermalization time scale we calculate the DM energy loss rate:

$$
\frac{d E}{d t}=-\xi n_{B} \sigma_{n} v \delta E
$$

where $n_{B}$ is the neutron number density in the center of the neutron star, $\delta E$ is the energy loss of the DM particle during each scattering event, and we use $\xi$ defined as in Eq. (3.11) to parameterize the neutron degeneracy effect on the DM thermalization process. The typical velocity and the momentum transfer $\delta p=\sqrt{2} m_{r} v$ fully determine $\xi$. However, unlike the capture case, where the velocity is set by the escape velocity $v_{e s c}$, the thermal equilibrium of DM particles and neutrons now sets $v \sim \sqrt{2 E_{t h} / m_{X}}$, where $E_{t h} \simeq 3 T / 2$ is the energy after thermalization. In the case of $m_{X} \gtrsim 1 \mathrm{GeV}$, $\delta p \sim \sqrt{2} m_{B} v \simeq 2.1 \times 10^{-5} \mathrm{GeV}\left(T / 10^{5} \mathrm{~K}\right)^{1 / 2}\left(100 \mathrm{GeV} / m_{X}\right)^{1 / 2}$, which is much smaller than $p_{F} \simeq 0.575 \mathrm{GeV}$. For $m_{X} \lesssim 1 \mathrm{GeV}$, the momentum transfer is given by $\delta p \sim \sqrt{2} m_{X} v \simeq 6.8 \times 10^{-5}\left(T / 10^{5} \mathrm{~K}\right)^{1 / 2}\left(m_{X} / 0.1 \mathrm{GeV}\right)^{1 / 2}$, which is again less than $p_{F}$. Therefore, the neutron degeneracy effect reduces the DM thermalization efficiency over the entire DM mass range, and $\xi$ is everywhere given by $\xi \simeq \delta p / p_{F}$.

To estimate the thermalization time scale, we solve Eq. (3.18) and get

$$
t_{t h} \simeq \frac{m_{X}^{2} m_{B} p_{F}}{4 \sqrt{2} n_{B} \sigma_{n} m_{r}^{3}} \frac{1}{E_{t h}}
$$

In the limit of $m_{X} \gtrsim 1 \mathrm{GeV}$, the thermalization time scale can be further simplified 
to $t_{t h} \simeq \sqrt{2} m_{X}^{2} p_{F} /\left(12 m_{B}^{2} n_{B} \sigma_{n} T\right)$. Taking typical values, we see

$$
t_{t h} \simeq 0.054 \text { years }\left(\frac{m_{X}}{100 \mathrm{GeV}}\right)^{2}\left(\frac{2.1 \times 10^{-45} \mathrm{~cm}^{2}}{\sigma_{n}}\right)\left(\frac{10^{5} \mathrm{~K}}{T}\right)
$$

If DM mass is less than $1 \mathrm{GeV}$, the thermalization time scale is given by

$$
t_{t h} \simeq 7.7 \times 10^{-5} \text { years }\left(\frac{0.1 \mathrm{GeV}}{m_{X}}\right)\left(\frac{2.1 \times 10^{-45} \mathrm{~cm}^{2}}{\sigma_{n}}\right)\left(\frac{10^{5} \mathrm{~K}}{T}\right)
$$

To derive constraints on scalar ADM from black hole formation, we will assume the captured scalar ADM follows the thermal distribution in the neutron star. This is only true when $t_{t h}$ is less than the neutron star age $\sim 10^{10}$ years. As we can see from Eqs. (3.20) and (3.21), light DM easily satisfies this condition. For heavy DM, $t_{t h}$ is not always less than the neutron star age. In the following discussion we first assume the DM reaches thermal equilibrium with neutrons, and then we check the consistency of this assumption.

After attaining thermal equilibrium, captured DM particles drift to the center of the star and form an isothermal distribution with the typical radius

$$
r_{t h}=\left(\frac{9 T}{4 \pi G \rho_{B} m_{X}}\right)^{1 / 2} \simeq 24 \mathrm{~cm}\left(\frac{T}{10^{5} K} \cdot \frac{100 \mathrm{GeV}}{m_{X}}\right)^{1 / 2}
$$

We can see that the captured DM particles very quickly occupy a very small region near the neutron star core.

\subsubsection{Self-gravitation and Black Hole Formation}

If the DM density is larger than the baryon density within the thermal radius $r_{t h}$, the DM particles can become self-gravitating. For a total DM mass $M_{X}=N_{X} m_{X}$ 
within a thermal radius $r_{t h}$, this condition is

$$
\frac{3 M_{X}}{4 \pi r_{t h}^{3}} \gtrsim \rho_{B}
$$

Therefore, the DM becomes self-gravitating once the total number of DM particles is larger than a critical number

$$
N_{\text {self }} \simeq 4.8 \times 10^{41}\left(\frac{100 \mathrm{GeV}}{m_{X}}\right)^{5 / 2}\left(\frac{T}{10^{5} \mathrm{~K}}\right)^{3 / 2}
$$

Recall the upper limit for the bosonic system given in Eq. (3.6) above which the zero point energy cannot prevent gravitational collapse:

$$
N_{\text {Cha }}^{\text {boson }} \simeq 1.5 \times 10^{34}\left(\frac{100 \mathrm{GeV}}{m_{X}}\right)^{2} .
$$

Thus, if the scalar ADM thermalizes and the mass satisfies $m_{X} \lesssim 10^{17} \mathrm{GeV}\left(T / 10^{5} \mathrm{~K}\right)^{3}$, we always have $N_{\text {self }} \gtrsim N_{C h a}^{\text {boson }}$. In this case, gravitational collapse occurs as soon as DM particles become self-gravitating in neutron stars.

\subsubsection{Bose-Einstein Condensation}

In the above discussion, we implicitly assumed that all captured scalar ADM particles followed a Maxwellian velocity distribution. At the extreme densities we are considering here, however, this minimal assumption is not necessarily satisfied. In particular, ensembles of bosonic particles at high densities exhibit novel statistical properties. If the central temperature of the neutron star falls below the critical temperature to form a Bose-Einstein condensate (BEC), the particles in the ground state condense and no longer follow the thermal distribution. We will now show that for light $\mathrm{ADM}$ this condensation increases the density and reduces the restriction on self-gravitation to such an extent that the number of ADM particles necessary 
for self-gravitation is less than the bosonic Chandrasekhar limit. Thus, gravitational collapse is set by $N_{C h a}^{b o s o n}$.

To check this sequence of events, we begin by noting that for a given bosonic DM number density $n_{X}$, the critical temperature to form the BEC is given by

$$
T_{c}=\frac{2 \pi}{m_{X}}\left[\frac{n_{X}}{\zeta(3 / 2)}\right]^{2 / 3}
$$

where $\zeta$ is the Riemann-Zeta function, $\zeta(3 / 2) \simeq 2.612$, and $n_{X}=3 N_{X} /\left(4 \pi r_{t h}^{3}\right)$. To see how likely it is that the captured ADM will form a BEC in the neutron star, we can estimate the critical ADM number as

$$
N_{X}=\zeta\left(\frac{3}{2}\right)\left(\frac{m_{X} T}{2 \pi}\right)^{3 / 2} \frac{4 \pi r_{t h}^{3}}{3} \simeq 1.0 \times 10^{36}\left(\frac{T}{10^{5} \mathrm{~K}}\right)^{3}
$$

where we have used Eq. (3.22). Therefore, if the total captured ADM in the neutron star is larger than $1.0 \times 10^{36}\left(T / 10^{5} \mathrm{~K}\right)^{3}$, some of captured ADM particles go to the ground state and form a BEC. This condition can be satisfied for a neutron star with relatively low central temperature as indicated by Eqs. (3.16) and (3.17) .

For $T<T_{c}$, the BEC forms and the number of particles in the condensed ground state is

$$
N_{X}^{0}=N_{X}\left[1-\left(\frac{T}{T_{c}}\right)^{3 / 2}\right] \simeq N_{X}-1.0 \times 10^{36}\left(\frac{T}{10^{5} \mathrm{~K}}\right)^{3} .
$$

Since these ground state particles effectively have zero temperature, they sink deep into the core of the neutron star. We can estimate the radius of distribution of the ground state by requiring the zero point energy equal the gravitational energy:

$$
r_{B E C}=\left(\frac{3}{8 \pi G m_{X}^{2} \rho_{B}}\right)^{1 / 4} \simeq 1.5 \times 10^{-5} \mathrm{~cm}\left(\frac{100 \mathrm{GeV}}{m_{X}}\right)^{1 / 2} .
$$

This is much smaller than $r_{t h}$, which indicates a much higher DM density. Thus, the ground state itself may become self-gravitating. The critical number for the 
self-gravity of the DM particles in the condensed state is

$$
N_{\text {self }}^{0}=\frac{4 \pi}{3} \frac{\rho_{B} r_{B E C}^{3}}{m_{X}} \simeq 1.0 \times 10^{23}\left(\frac{100 \mathrm{GeV}}{m_{X}}\right)^{5 / 2}
$$

Once the number of DM particles in the ground state is larger than $N_{\text {self }}^{0}$, these ground state particles become self-gravitating. Since $N_{\text {self }}^{0}$ is less than $N_{\text {self }}$, the onset of self-gravity is marked by $N_{\text {self }}^{0}$ instead of $N_{\text {self }}$ in conditions where a BEC forms. As indicated above, this leads to qualitatively different behavior as compared to the case when a BEC does not form, since now $N_{\text {self }}^{0}$ can be less than $N_{C h a}^{\text {boson }}$. If this is the case, as soon as a condensed ADM system reaches the Chandrasekhar limit it will undergo gravitational collapse.

For this effect to be important, $N_{X}^{0}$ has to grow larger than the Chandrasekhar limit for a bosonic system, so that the condition for black hole formation of the BEC becomes $N_{X}^{0} \gtrsim N_{C h a}^{\text {boson }}$. By using Eq. (3.28), we get a lower limit on the total DM number $N_{X}$,

$$
\begin{aligned}
N_{\text {BEC }} & =N_{\text {Cha }}^{\text {boson }}+1.0 \times 10^{36}\left(\frac{T}{10^{5} \mathrm{~K}}\right)^{3} \\
& \simeq 1.5 \times 10^{34}\left(\frac{100 \mathrm{GeV}}{m_{X}}\right)^{2}+1.0 \times 10^{36}\left(\frac{T}{10^{5} \mathrm{~K}}\right)^{3} .
\end{aligned}
$$

We can see that the value of the right-hand side of Eq. (3.31) is less than $N_{\text {self }}$ if the DM mass $m_{X} \lesssim 1.9 \times 10^{4} \mathrm{GeV}\left(4.7 \times 10^{3} \mathrm{GeV}\right)$ for a central temperature $T=10^{5} \mathrm{~K}$ $\left(10^{6} \mathrm{~K}\right)$. In the situation where this condensation can occur, the BEC shortens the time scale for the black hole formation for the scalar ADM in the low mass range.

\subsection{Hawking Radiation and Destruction of the Host Star}

During the collapse process, the gravitational contraction releases energy which can be absorbed by neutrons through DM-neutron scattering. This cooling mech- 
anism is so efficient that eventually the DM sphere collapses to a black hole [36]. Once a black hole is formed at the center of the neutron star, it will rapidly capture the baryonic matter of the neutron star. Hawking radiation will also be active, reducing the mass of the black hole and possibly heating the remaining DM. Finally, the black hole may also consume the ambient DM particles, which can be crucial for the stability of the black hole. Here, we analyze the relative contributions of these effects, and we see that in the majority of our parameter space the black hole will grow and eventually consume the host neutron star. Once the physics of the accretion is properly considered, we find that Hawking radiation could be important for highand intermediate-mass ADM.

For a black hole with mass $M_{B H}$, the differential equation that governs the rate of change of mass is

$$
\frac{d M_{B H}}{d t} \simeq 4 \pi \lambda_{s}\left(\frac{G M_{B H}}{v_{s}^{2}}\right)^{2} \rho_{B} v_{s}-\frac{1}{15360 \pi G^{2} M_{B H}^{2}}+\left(\frac{d M_{B H}}{d t}\right)_{D M}
$$

The second term of Eq. (3.32) represents the Hawking radiation rate, while the third term is the accretion rate of ambient DM particles. The first term of right-hand side of Eq. (3.32) is the Bondi-Hoyle accretion rate, in which $v_{s}=\sqrt{d P / d \rho}$ is the sound speed and $\lambda_{s}$ is the accretion eigenvalue for the transonic solution. To determine $v_{s}$ and $\lambda_{s}$, we characterize the equation of state of the neutrons by $P=K \rho^{\gamma}$, where $K$ and $\gamma$ are constant. For a non-relativistic degenerate neutron gas, which is a good approximation for neutrons in the neutron star, we have $\gamma=5 / 3$ and $K=$ $3^{2 / 3} \pi^{4 / 3} /\left(5 m_{B}^{8 / 3}\right)[40]$. We estimate the sound speed as $v_{s}=\sqrt{K \gamma \rho^{\gamma-1}} \sim 10^{5} \mathrm{~km} / \mathrm{s}$, where we take $\rho \sim 1.4 \times 10^{15} \mathrm{~g} / \mathrm{cm}^{3}$. The accretion constant is given by $\lambda_{s}=$ $(1 / 2)^{(\gamma+1) /(2 \gamma-2)}[(5-3 \gamma) / 4]^{-(5-3 \gamma) /(2 \gamma-2)}=0.25[40]$. 


\subsubsection{Black Hole Mass without Bose-Einstein Condensation}

For large ADM mass, we found above that $N_{\text {self }}<N_{B E C}$ and a black hole forms without the assistance of a BEC. After formation of the black hole, the neutron star continues capturing DM particles. These newly captured DM particles eventually sink to the center of the neutron star and distribute themselves within $r_{t h}$. In principle, the black hole can increase its mass by capturing these additional DM particles. However, we find that this capture rate is very small and $\left(d M_{B H} / d t\right)_{D M}$ is negligible. This is because, for a non-relativistic particle moving towards the black hole, its impact parameter must be less than $b_{\max }=4 G M_{B H} / v_{\infty}[40]$ to penetrate the angular momentum barrier and fall into the black hole. Here, $v_{\infty}$ is the particle's velocity when it is far away from the black hole.

Taking $M_{B H} \sim m_{X} N_{\text {self }}$ and $v_{\infty} \sim \sqrt{3 T / m_{X}}$, we can estimate $b_{\max }$ as

$$
b_{\max } \sim 1.6 \times 10^{-5} \mathrm{~cm}\left(\frac{10 \mathrm{TeV}}{m_{X}}\right)\left(\frac{T}{10^{5}}\right) \text {, }
$$

which is much smaller than the thermal radius $r_{t h} \simeq 2.4 \mathrm{~cm}\left(T / 10^{5} K\right)\left(10 \mathrm{TeV} / m_{X}\right)^{1 / 2}$. Therefore the majority of DM particles captured after formation of the black hole do not fall directly into the black hole. The remaining DM particles orbit the black hole at a distance of order $r_{t h}$; the black hole gains mass from these particles at a rate set by the collisionless spherical accretion approximation [40]. We find that the DM accretion rate is much less than the baryon accretion rate, so we can safely ignore the $\left(d M_{B H} / d t\right)_{D M}$ term in this case, and we obtain a critical initial black hole mass:

$$
M_{B H}^{\text {crit }} \simeq 1.2 \times 10^{37} \mathrm{GeV}
$$

Without a BEC, the initial black hole mass is $M_{B H} \sim N_{\text {self }} m_{X}$. If we demand $N_{\text {self }} m_{X} \gtrsim M_{B H}^{c r i t}$, we find that for $m_{X} \lesssim 2.6 \times 10^{6} \mathrm{GeV}\left(T / 10^{5} \mathrm{~K}\right)$ the Hawking 
radiation has a longer time scale than the accretion process. Hence in this mass range the black hole will continuously accrete baryonic matter until the neutron star is consumed entirely.

\subsubsection{Black Hole Mass with Bose-Einstein Condensation}

For low-mass ADM, particles in the BEC ground state form a black hole. We must check the mass above which the black hole evaporates. If we naively ignore the term $\left(d M_{B H} / d t\right)_{D M}$ and demand $M_{B H} \sim m_{X} N_{C h a}^{\text {boson }} \gtrsim M_{B H}^{c r i t}$, we find that the black hole mass increases only for the DM mass less than $\sim 13 \mathrm{GeV}$. If so, the constraint is valid for $m_{X} \lesssim 13 \mathrm{GeV}$. But in contrast to the non-BEC case, $\left(d M_{B H} / d t\right)_{D M}$ may have an important effect on the black hole mass evolution, and we find that the bound can be sensitive to masses higher than $\sim 13 \mathrm{GeV}$. We detail our reasoning below.

Since the black hole forms only from ADM particles in the ground state, the remaining ADM particles follow an isothermal distribution with a radius $r_{t h}$. As discussed above, the thermally distributed DM particles do not fall into the black hole, and so the phase space of the non-BEC state is still completely occupied. Hence, if any more ADM particles are introduced to the thermal region, a new BEC ground state must form in the center of the star. In this way, the introduction of more DM particles into the thermal radius essentially forces the formation of a BEC ground state. Before and after the mini black hole forms, the neutron star continuously captures ADM particles. All of the captured ADM particles will eventually thermalize, sink to the center of the neutron star, and prompt the formation of a new BEC state.

If the thermalization time scale is shorter than the evaporation time scale of the mini black hole, the black hole can always efficiently accrete ADM particles in the new BEC state. Taking the initial black hole mass as $M_{B H} \sim m_{X} N_{C h a}^{\text {boson }}=1 /\left(G m_{X}\right)$, we can conservatively estimate the black hole evaporation time scale in the absence of particle accretion as $t_{\text {haw }} \simeq 15360 \pi G^{2} M_{B H}^{3} / 3 \simeq 5 \times 10^{4}$ years $\left(100 \mathrm{GeV} / m_{X}\right)^{3}$. 
This is much longer than the thermalization time scale given in Eq. (3.20). Since all newly-captured ADM particles eventually go to the ground state after the amount of time it takes them to thermalize, the rate at which ADM particles fall into the BEC ground state per unit time is given by the capture rate $C_{B}$. To check whether or not the ADM particles in the BEC state feed the black hole efficiently we calculate the maximal impact parameter $b_{\max }$ and compare it with the distribution radius $r_{B E C}$. With $M_{B H} \sim 1 /\left(G m_{X}\right)$ and $v_{\infty} \sim 1 /\left(m_{X} r_{B E C}\right)$, we have

$$
b_{\max } \sim 4 r_{B E C},
$$

where $r_{B E C}$ is given by Eq. (3.29). Since $b_{\max } \gtrsim r_{B E C}$, we see that the black hole can efficiently consume the BEC. This occurs at a rate given by $\left(d M_{B H} / d t\right)_{D M} \sim m_{X} C_{B}$ :

$$
\left(\frac{d M_{B H}}{d t}\right)_{D M} \simeq 2.3 \times 10^{36} \mathrm{GeV} / \text { year }\left(\frac{\rho_{X}}{10^{3} \mathrm{GeV} / \mathrm{cm}^{3}}\right)\left(\frac{\sigma_{X B}}{2.1 \times 10^{-45} \mathrm{~cm}^{2}}\right) .
$$

This new source of accretion overwhelms the Hawking radiation, which is emitted at the rate $\left(d M_{B H} / d t\right)_{h w} \sim 10^{27} \mathrm{GeV} /$ year $\left(m_{X} / 100 \mathrm{GeV}\right)^{2}$. Thus, in the BEC case our constraints can be sensitive to scalar ADM with mass much higher than the naive estimate, $m_{X} \sim 13 \mathrm{GeV}$.

In this discussion, we have assumed that the black hole formation and Hawking radiation do not destroy the BEC state. However, one may note that the black hole radiates as a blackbody at a very high temperature, $T_{\text {haw }}=\left(8 \pi G M_{B H}\right)^{-1} \sim m_{X} / 8 \pi$. If this Hawking radiation is emitted solely as relativistic particles of the standard model such as photons and neutrinos, these particles will heat the neutrons which in turn heat the ADM. We may estimate the change in neutron temperature by assuming that all of the initial rest mass energy of the black hole goes into heat. We see that in this case equipartition of energy requires that $\Delta T \sim m_{X} N_{C h a}^{\text {boson }} / N_{B} \simeq$ $1.0 \times 10^{-7} \mathrm{~K}\left(10 \mathrm{GeV} / m_{X}\right)$, and there is no change in the thermal ADM distribution. 
On the other hand, if the energy produced by the Hawking radiation is efficiently transferred to DM particles, the situation may change. For example, the DM may couple to some light mediator particles, which can be produced by the Hawking radiation. These light mediators may heat the ADM directly and transfer the black hole energy to the thermal energy of ADM particles. In this case, the calculation is rather complicated and a detailed analysis is beyond the scope of the current paper. Here we give a conservative estimate by assuming that all of the initial black hole mass goes to the thermal energy of DM particles. The equipartition of energy gives $\Delta T \sim m_{X} N_{\text {Cha }}^{\text {boson }} / N_{t h} \simeq 1.7 \times 10^{14} \mathrm{~K}\left(10 \mathrm{GeV} / m_{X}\right)\left(T / 10^{5} \mathrm{~K}\right)^{-3}$, where the number of remaining non-BEC particles is $N_{t h}=1.0 \times 10^{36}\left(T / 10^{5} \mathrm{~K}\right)^{3}$, from Eq. (3.31). This heating will greatly affect the thermal distribution of the scalar ADM particles. Since $r_{t h} \propto \sqrt{T}$, the phase space will expand greatly, and the newly-captured ADM particles do not form the BEC state. Thus, the black hole constraints are lifted in this case.

In practice, for the heating process to occur ADM particle must couple to some light mediator particles, because the black hole can only produce particles with mass much less than $T_{\text {haw }} \sim m_{X} / 8 \pi$. On the other hand, the presence of the light mediator may also mediate DM self-interactions, and the observed ellipticity of DM halos places a lower bound on the mediator mass [43]. As an example, if $m_{X} \sim 13 \mathrm{GeV}$, the halo shape constraint requires the mediator mass larger than $\sim 40 \mathrm{MeV}$ [44]. Thus, for the scalar ADM with mass $m_{X} \sim 13 \mathrm{GeV}$ the existence of a mediator with mass in the range $\sim 40 \mathrm{MeV}-0.5 \mathrm{GeV}$ may help avoid the black hole formation constraints while remaining consistent with the halo shape bound. We can see that the constraints for $m_{X} \gtrsim 13 \mathrm{GeV}$ in the BEC case are rather model-dependent, and we specify these regions in our plots.

Now we estimate the destruction time scale. If the initial black hole mass exceeds the critical value $M_{B H}^{c r i t}$, the time scale to destroy the neutron star is set by the Bondi- 
Hoyle rate:

$$
t \sim \frac{v_{s}^{3}}{\pi G^{2} \rho_{B} M_{B H}} \simeq 2.3 \times 10^{-5} \mathrm{~s}\left(\frac{M_{\odot}}{M_{B H}^{i}}\right),
$$

where we take $v_{s} \sim 10^{5} \mathrm{~km} / \mathrm{s}$ and $M_{\odot}$ is the mass of sun. Therefore, the characteristic time scale is $t \sim 17$ years $\left(m_{X} / 100 \mathrm{GeV}\right)^{3 / 2}\left(10^{5} \mathrm{~K} / T\right)^{3 / 2}$ and $5.4 \times 10^{6}$ years $\left(m_{X} / \mathrm{GeV}\right)$ for the non-BEC case and BEC case, respectively, which we note is much shorter than the typical old neutron star age $\sim 10^{10}$ years.

\subsection{Observational Constraints}

So far, we have gone through the conditions for the captured scalar ADM particles to form a black hole. Such a black hole can destroy the host neutron star. We can see that these conditions are easily satisfied if the ADM particles have a sizable scattering cross section with neutrons. However, we observe many old neutron stars near the solar system and in globular clusters. Therefore, these observed old neutrons stars constrain the ADM-neutron scattering cross section. In this section, we proceed to derive upper bounds on ADM-neutron scattering cross section for a given ADM mass. In Sections 3.6.1 (3.6.2), we first derive the constraints on $\sigma_{n}$ by assuming fiducial neutron star parameters for the case without (with) Bose-Einstein condensation. In Section 3.6.3, we apply these bounds to observed neutron stars in the solar neighborhood and in the globular cluster M4.

\subsubsection{Constraints on DM-neutron cross section without Bose-Einstein condensation}

We first discuss constraints in the absence of BEC formation; this gives rise to conservative constraints in the low mass range but is more stringent for high masses, where BECs do not readily form. Since in this case $N_{\text {self }}>N_{\text {Cha }}^{\text {boson }}$ for $m_{X} \lesssim 10^{17} \mathrm{GeV}\left(T / 10^{5} \mathrm{~K}\right)^{3}$, gravitational collapse occurs as soon as DM particles 
start self-gravitating. In order to avoid the destruction of neutron stars, we demand $N_{X}<N_{\text {self }}$ and get an upper bound on DM-neutron scattering cross section $\sigma_{n}$. For DM with mass $m_{X} \gtrsim 1 \mathrm{GeV}$ we derive the following bound on $\sigma_{n}$ using Eqs. (3.16) and (3.24) and requiring that the host NS is not destroyed over the course of its lifetime $t$ :

$$
\sigma_{n}<4.4 \times 10^{-48} \mathrm{~cm}^{2}\left(\frac{10^{3} \mathrm{GeV} / \mathrm{cm}^{3}}{\rho_{X}} \cdot \frac{10^{10} \text { years }}{t}\right)\left(\frac{100 \mathrm{GeV}}{m_{X}} \cdot \frac{T}{10^{5} \mathrm{~K}}\right)^{3 / 2}
$$

For $m_{X} \lesssim 1 \mathrm{GeV}$, the bound on $\sigma_{n}$ is

$$
\sigma_{n}<9.3 \times 10^{-46} \mathrm{~cm}^{2}\left(\frac{10^{6} \mathrm{GeV} / \mathrm{cm}^{3}}{\rho_{X}} \cdot \frac{10^{10} \text { years }}{t}\right)\left(\frac{0.1 \mathrm{GeV}}{m_{X}}\right)^{5 / 2}\left(\frac{T}{10^{5} \mathrm{~K}}\right)^{3 / 2}
$$

Since the capture rate saturates when the DM-neutron scattering cross section is larger than $\sigma_{\max } \simeq 2.1 \times 10^{-45} \mathrm{~cm}^{2}$, the upper bound on $\sigma_{n}$ is only valid when the value of the right-hand side of Eq. (3.38) and Eq. (3.39) is smaller than $\sigma_{\max }$. Because the capture and thermalization processes do not distinguish between spin-dependent and spin-independent cross sections, the bound on $\sigma_{n}$ applies to both cases.

We depict the constraints from requiring the NS survival in the left panel of Fig. (3.1) with various values of the DM density. We take the central temperature as $10^{5} \mathrm{~K}$ and neutron star age to be $10^{10}$ years. The most prominent qualitative feature, the sharp vertical cutoff, corresponds to cross sections $\sigma_{n} \simeq 2.1 \times 10^{-45} \mathrm{~cm}^{2}$. Here, the geometric cross section limits the capture of DM particles, so we cannot constrain the interaction cross section for the mass below the cutoff. Furthermore, without a BEC, we can constrain scalar ADM with mass $m_{X} \lesssim 100 \mathrm{MeV}$ only if the DM density $\rho_{X} \gtrsim 10^{6} \mathrm{GeV} / \mathrm{cm}^{3}$. One may find regions with such high DM density near the galactic center. As we discussed before, if the DM mass is less than $\sim 1 \mathrm{GeV}$, the capture rate is reduced due to the neutron degeneracy effect, which is indicated by the change in slope of the curve with $\rho_{X}=10^{6} \mathrm{GeV} / \mathrm{cm}^{3}$ when $m_{X} \lesssim 1 \mathrm{GeV}$. 
For DM mass $m_{X} \gtrsim 10^{6} \mathrm{GeV}$, the factor $B^{2}$ in Eq. (4.1) falls below one and the capture probability through a single scatter becomes lower, as shown by the bump in the left panel of the figure. In the hatched region, DM particles cannot thermalize with the surrounding neutrons within the age of neutron star as discussed in Section IV A. In this region, the captured DM does not necessarily distribute within the small thermal radius of the neutron star core, and the bound does not apply. In the cross-hatched region, the initial black hole mass is so small that it can evaporate due to the Hawking radiation as shown in Section V A. In the square-hatched region, the Hawking radiation may interfere with DM accretion, and the black hole will evaporate in some case as discussed in Section V B.

We can also estimate the analogous bound from white dwarf stars. Typical white dwarf parameters are [45]: mass $\sim 0.7 M_{\odot}$, radius $\sim 6.3 \times 10^{3} \mathrm{~km}$, density $\sim 10^{6} \mathrm{~g} / \mathrm{cm}^{3}$, central temperature $\sim 10^{7} \mathrm{~K}$ and escape velocity $\sim 6 \times 10^{3} \mathrm{~km} / \mathrm{s}$. White dwarfs are composed of carbon and oxygen; we make the conservative assumption that the white dwarf is entirely composed of carbon. We find that the white dwarf bound on $\sigma_{n}$ is about nine orders of magnitude weaker than the limit derived for typical neutron stars.

\subsubsection{Constraints on DM-neutron cross section with Bose-Einstein con- densation}

If the captured scalar ADM does form a BEC, the constraints on the DM-neutron

cross section become stronger. This is because $N_{B E C} \lesssim N_{\text {self }}$ for the DM mass less than a few $\mathrm{TeV}$, dependent on the central temperature $T$. In this case, self-gravitation and gravitational collapse will occur more quickly due to the heightened density of the ADM. 

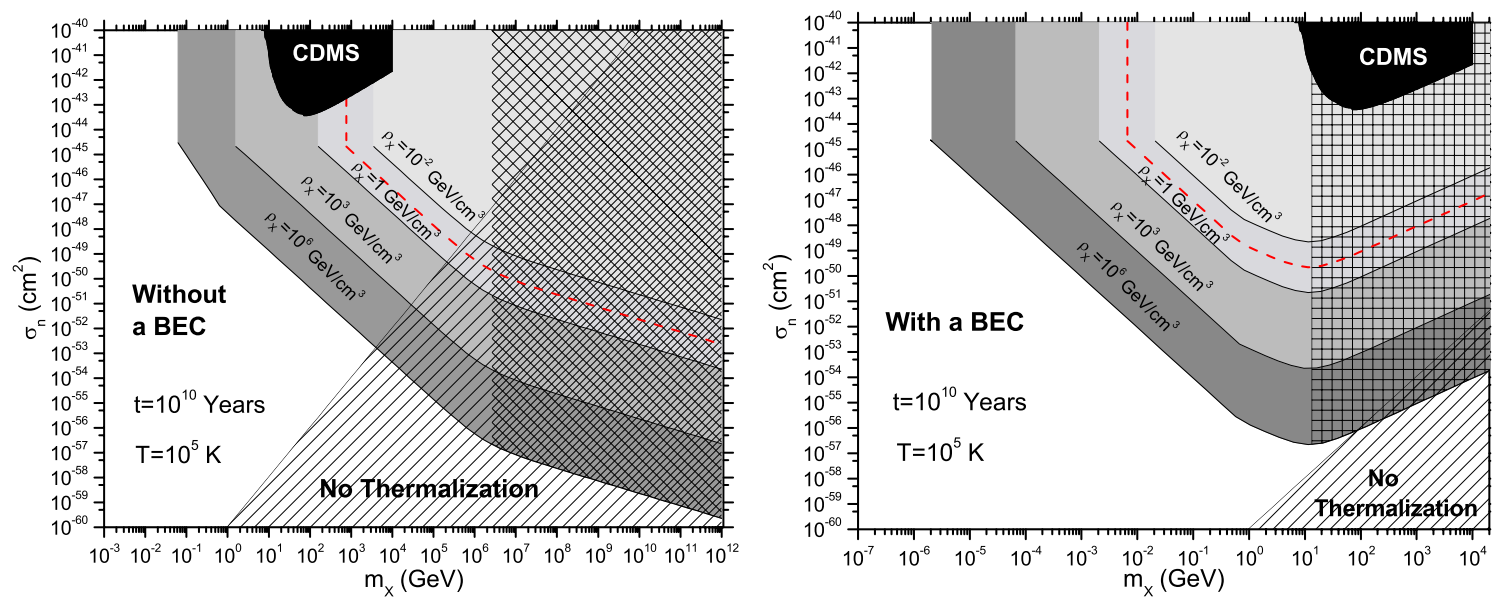

Figure 3.1: Regions (gray) of DM-neutron scattering cross-section in which accumulated scalar ADM forms a black hole. We have $\rho_{X}=0.1 \mathrm{~cm}^{3} / \mathrm{s}$ along the dotted red lines, which is approximately the DM density in the solar neighborhood. In the left panel, we assume a Bose-Einstein condensate (BEC) does not form and all captured DM particles become self-gravitating and collapse. In the right panel, we assume a Bose-Einstein condensate (BEC) does form and DM particles in the BEC ground state collapse and form a black hole. We take the neutron star age to be $10^{10}$ years with a central temperature $10^{5} \mathrm{~K}$. In the diagonally shaded regions, DM particles cannot thermalize with neutrons within the age of neutron star. In the diagonally cross-hatched region, the black hole can evaporate due to the Hawking radiation. In the square-hatched regions, Hawking radiation may interfere with DM accretion by heating the thermalized DM, and in some cases the black hole can evaporate (see discussion in Section V). In these hatched regions, the bounds are lifted. The black regions are excluded by recent CDMS results (SI) [3]. 
For $m_{X} \gtrsim 1 \mathrm{GeV}$, the requirement $N_{X} \lesssim N_{B E C}$ gives:

$$
\begin{aligned}
\sigma_{n} \lesssim 9.1 & \times 10^{-54} \mathrm{~cm}^{2}\left(\frac{10^{3} \mathrm{GeV} / \mathrm{cm}^{3}}{\rho_{X}} \cdot \frac{10^{10} \text { years }}{t}\right)\left(\frac{m_{X}}{100 \mathrm{GeV}}\right) \\
\times & {\left[1.5 \times 10^{-2}\left(\frac{100 \mathrm{GeV}}{m_{X}}\right)^{2}+\left(\frac{T}{10^{5} \mathrm{~K}}\right)^{3}\right] }
\end{aligned}
$$

In the case of $m_{X} \lesssim 1 \mathrm{GeV}$, the upper limit on $\sigma_{n}$ is given by

$$
\begin{gathered}
\sigma_{n} \lesssim 6.2 \times 10^{-56} \mathrm{~cm}^{2}\left(\frac{10^{3} \mathrm{GeV} / \mathrm{cm}^{3}}{\rho_{X}} \cdot \frac{10^{10} \text { years }}{t}\right) \\
\times\left[1.5 \times 10^{4}\left(\frac{0.1 \mathrm{GeV}}{m_{X}}\right)^{2}+\left(\frac{T}{10^{5} \mathrm{~K}}\right)^{3}\right]
\end{gathered}
$$

Again, we derive bounds by requiring that the host star is not destroyed by the gravitational collapse of the ADM.

In the right panel of Fig. (3.1), we display the DM-neutron scattering cross section with various values of the DM density that satisfy $N_{X} \gtrsim N_{B E C}$. For $m_{X} \lesssim 10 \mathrm{GeV}$,

the BEC forms before the Chandrasekhar mass is reached, while for $m_{X} \gtrsim 10 \mathrm{GeV}$, the Chandrasekhar mass is reached before the BEC forms, so that collapse of the DM to a black hole occurs as soon as the BEC forms. The change in the slope of the curves around $m_{X} \sim 1 \mathrm{GeV}$ is a combination of this effect with a decreased capture efficiency below $m_{X} \sim 1 \mathrm{GeV}$. We can see the formation of the BEC significantly improves the bound for light ADM. As an example, we see that for $\rho_{X}=1 \mathrm{GeV} / \mathrm{cm}^{3}$, BEC formation strengthens the constraint as long as $m_{X} \lesssim 13 \mathrm{GeV}$, while for higher masses the entire mass of ADM becomes self-gravitating before BEC formation occurs.

\subsubsection{Constraints from observed pulsars}

Now we consider the observations of a few relatively cold and old neutron stars that can provide tests of this effect. PSR J0437-4715 is a nearby pulsar at a distance of about $139 \pm 3 \mathrm{pc}$ from the solar system. The surface temperature is $T_{e}=1.2 \times$ 

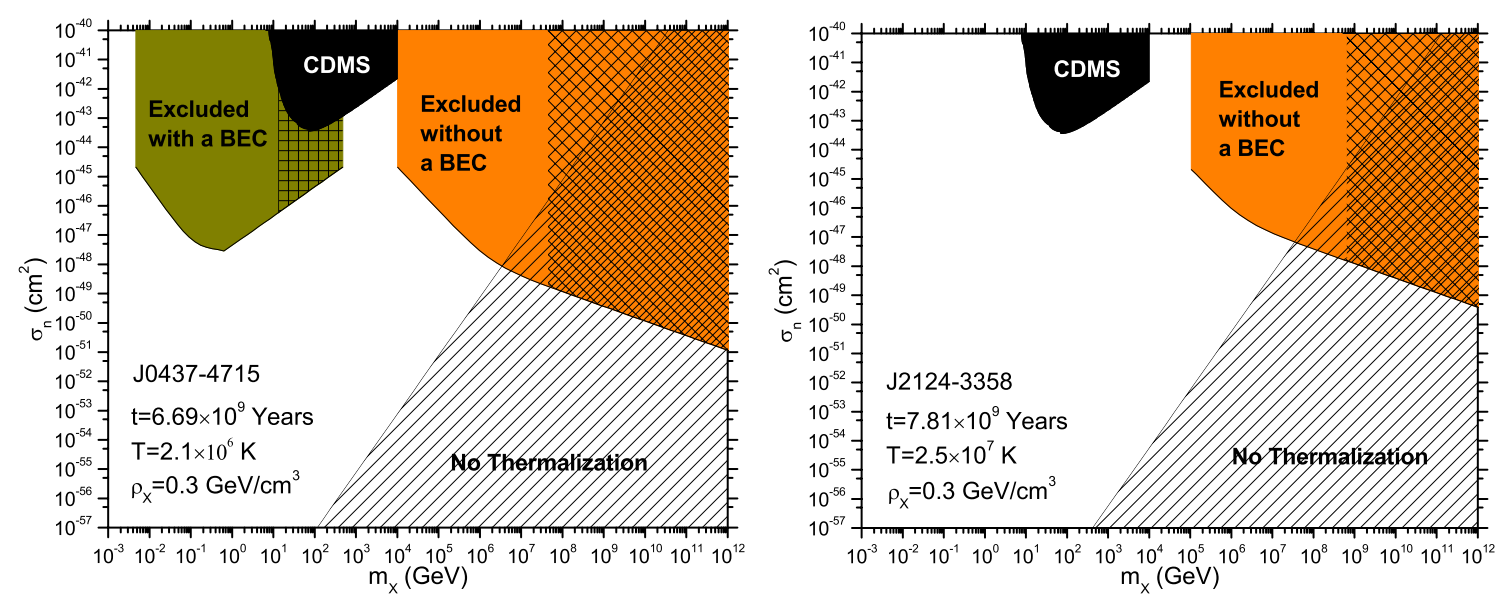

Figure 3.2: Regions (colored) excluded by the nearby pulsars J0437-4715 (left) and J2124-3358 (right). The shaded, diagonal and square cross-hatched, and black regions are as in Fig. (3.1). In hatched regions, the bounds are lifted.

$10^{5} \mathrm{~K}$ [46]. When its secular motion is accounted for, calculations indicate its age is $6.69 \times 10^{9}$ years [47]. Another nearby pulsar is PSR J2124-3358, located 270 pc away from us with a surface temperature $T_{e}<4.6 \times 10^{5} \mathrm{~K}$ [46]. Its age is $7.81 \times 10^{9}$ years [47]

For these nearby pulsars, we can calculate the central temperature from the surface temperature $T_{e}$ by using the analytical formula [48]

$$
T \simeq 1.288 \times 10^{8} \mathrm{~K}\left[\frac{10^{14} \mathrm{~cm} / \mathrm{s}}{g_{s}}\left(\frac{T_{e}}{10^{6} \mathrm{~K}}\right)^{4}\right]^{0.455}
$$

where $g_{s}=G M_{n} / R_{n}^{2}$ is the surface gravity and we take $g_{s} \simeq 1.7 \times 10^{14} \mathrm{~cm} / \mathrm{s}^{2}$ for $M_{n}=1.44 M_{\odot}$ and $R_{n}=10.6 \mathrm{~km}$. We find the central temperature is $2.1 \times 10^{6} \mathrm{~K}$ and $2.5 \times 10^{7} \mathrm{~K}$ for J0437-4715 and J2124-3358, respectively. We take the ambient DM density to be $0.3 \mathrm{GeV} / \mathrm{cm}^{3}$, because these pulsars are in our relative neighborhood.

In Fig. (3.2), we show the constraints on the DM-nucleon scattering cross section of the scalar ADM from the nearby pulsars J0437-4715 (left panel) and J2124-3358 (right panel). We can see that J0437-4715 can constrain scalar ADM with $m_{X} \gtrsim 10 \mathrm{TeV}$ without a BEC. With the formation of a BEC, it is also sensitive to the mass range 
$m_{X} \sim 5 \mathrm{MeV}-13 \mathrm{GeV}$. The captured scalar ADM cannot form a BEC in the pulsar J2124-3358. This is because it has a relatively high central temperature, and the formation of a BEC requires a DM-nucleon cross section larger than the saturation cross section $\sigma_{\max } \simeq 2.1 \times 10^{-45} \mathrm{~cm}^{2}$.

Since the bound is sensitive to the DM density, we also consider neutron stars in regions with high $\rho_{X}$. Globular clusters possibly offer this type of environment, and observations of Pulsar B1620-26 place it in the globular cluster M4 [49] with an age of $2.82 \times 10^{8}$ years [46]. Since it is far away from us, its surface temperature is unknown, and we are not able to calculate its central temperature. In our analysis, we take $T=10^{6} \mathrm{~K}$ as a reasonable approximation due to its advanced age. We take $\rho_{X}=10^{3} \mathrm{GeV} / \mathrm{cm}^{3}$ for the DM density and $\bar{v}=20 \mathrm{~km} / \mathrm{s}$, motivated by discussions in Refs. $[25,38]$. Note that the exact value of DM density in globular clusters is unknown. Globular clusters are baryon-dominated systems, and currently there is no evidence that DM is present in these systems; see Ref. [50] for simulations of DM content in globular clusters. In Fig. (3.3), we show the constraints on the DM-nucleon scattering cross section of scalar ADM from the pulsar B1620-26 in the globular cluster

M4. Note that when the DM mass is larger than $\sim 4.7 \times 10^{3} \mathrm{GeV}, N_{B E C} \gtrsim N_{\text {self }}$ and all captured DM particles collapse before a BEC forms.

\subsection{Conclusions}

We have studied the consequences of scalar ADM accumulation in neutron stars. Neutron stars have high density and are ideal objects for capturing DM at high rates. Since ADM does not self-annihilate, a high mass of DM can accrete in the neutron star, and, lacking Fermi degeneracy pressure, rapidly self-gravitate and exceed the Chandrasekhar limit. Furthermore, the formation of a BEC increases the density of the ADM by several orders of magnitude, which greatly accelerates the onset of gravitational collapse and considerably strengthens the constraints in certain regions 


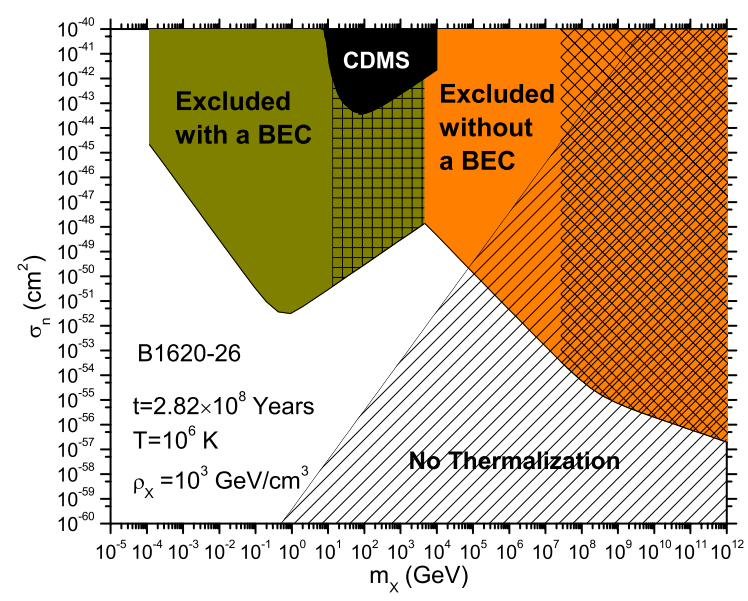

Figure 3.3: Regions (colored) excluded by the pulsar B1620-26 in the globular cluster M4. Note the globular cluster M4 is a baryon-dominated system, and there is no evidence for the presence of dark matter. Here, we take $\rho_{X}=$ $10^{3} \mathrm{GeV} / \mathrm{cm}^{3}$ motivated by numerical results in Refs. [25,38]. The shaded, diagonal and square cross-hatched, and black regions are as in Fig. (3.1). In hatched regions, the bounds are lifted.

of parameter space. For high dark matter mass, which corresponds to a low initial black hole mass, Hawking radiation can weaken the constraints. In the absence of light messenger particles, this effect is quite limited in scope, but if the black hole can heat the ADM directly it may affect a larger range of parameters.

We have computed the size of all of these effects and found that some presently observed pulsars constrain scalar ADM far more tightly than what is currently possible with direct detection experiments. These constraints are stronger even than the upcoming generation of experiments. We also note that these constraints can be significantly improved in the future with observations of old pulsars in regions of DM density greater than $10^{3} \mathrm{GeV} / \mathrm{cm}^{3}$.

Note: Since the first version of our paper appeared, [51] was written. In the case where a BEC forms and Hawking radiation is important, the authors of [51] find that scalar ADM with mass $\sim 0.75 \mathrm{MeV}-16 \mathrm{GeV}$ is excluded by nearby neutron stars if the scattering cross section with neutrons is sizable. Our results exclude scalar ADM 
in the mass range $\sim 5 \mathrm{MeV}-13 \mathrm{GeV}$ in the same scenario. The discrepancy in the low mass limit comes about because we take into account the degeneracy effects on the capture rate. This is important for DM mass below $\sim 1 \mathrm{GeV}$. 


\section{Bibliography}

[1] G. Jungman, M. Kamionkowski, K. Griest, Phys. Rept. 267, 195-373 (1996). [hep-ph/9506380]. G. Bertone, D. Hooper, J. Silk, Phys. Rept. 405, 279-390 (2005). [hep-ph/0404175]. J. L. Feng, [arXiv:1003.0904 [astro-ph.CO]].

[2] S. Nussinov, Phys. Lett. B165, 55 (1985). S. M. Barr, R. S. Chivukula and E. Farhi, Phys. Lett. B 241, 387 (1990). S. M. Barr, Phys. Rev. D 44, 3062 (1991). D. B. Kaplan, Phys. Rev. Lett. 68, 741 (1992).

[3] R. Kitano and I. Low, Phys. Rev. D 71, 023510 (2005) [arXiv:hep-ph/0411133].

[4] D. E. Kaplan, M. A. Luty and K. M. Zurek, Phys. Rev. D 79, 115016 (2009) [arXiv:0901.4117 [hep-ph]].

[5] H. An, S. L. Chen, R. N. Mohapatra and Y. Zhang, JHEP 1003, 124 (2010) [arXiv:0911.4463 [hep-ph]]. P. H. Gu, Phys. Rev. D 81, 095002 (2010) [arXiv:1001.1341 [hep-ph]]. B. Feldstein and A. L. Fitzpatrick, JCAP 1009, 005 (2010) [arXiv:1003.5662 [hep-ph]]. J. Shelton and K. M. Zurek, Phys. Rev. D 82, 123512 (2010) [arXiv:1008.1997 [hep-ph]]. H. Davoudiasl, D. E. Morrissey, K. Sigurdson and S. Tulin, Phys. Rev. Lett. 105, 211304 (2010) [arXiv:1008.2399 [hep-ph]]. N. Haba and S. Matsumoto, arXiv:1008.2487 [hep-ph]. E. J. Chun, arXiv:1009.0983 [hep-ph]. P. H. Gu, M. Lindner, U. Sarkar and X. Zhang, arXiv:1009.2690 [hep-ph]. M. Blennow, B. Dasgupta, E. Fernandez-Martinez and N. Rius, arXiv:1009.3159 [hep-ph]. J. McDonald, arXiv:1009.3227 [hep-ph]. S. R. Behbahani, M. Jankowiak, T. Rube and J. G. Wacker, arXiv:1009.3523 [hep-ph]. L. J. Hall, J. March-Russell and S. M. West, arXiv:1010.0245 [hepph]. R. Allahverdi, B. Dutta and K. Sinha, arXiv:1011.1286 [hep-ph]. B. Dutta and J. Kumar, arXiv:1012.1341 [hep-ph]. J. J. Heckman and S. J. Rey, 
arXiv:1102.5346 [hep-th]. M. T. Frandsen, S. Sarkar and K. Schmidt-Hoberg, arXiv:1103.4350 [hep-ph].

[6] T. Cohen, D. J. Phalen, A. Pierce and K. M. Zurek, Phys. Rev. D 82, 056001 (2010) [arXiv:1005.1655 [hep-ph]].

[7] Z. Kang, J. Li, T. Li, T. Liu, J. Yang, [arXiv:1102.5644 [hep-ph]].

[8] R. Bernabei et al. [DAMA Collaboration], Eur. Phys. J. C 56, 333 (2008) [arXiv:0804.2741 [astro-ph]].

[9] C. E. Aalseth et al. [CoGeNT collaboration], arXiv:1002.4703 [astro-ph.CO].

[10] D. Hooper, J. March-Russell, S. M. West, Phys. Lett. B605, 228-236 (2005). [hep-ph/0410114]. S. B. Gudnason, C. Kouvaris, F. Sannino, Phys. Rev. D74, 095008 (2006). [hep-ph/0608055]. R. Foadi, M. T. Frandsen, F. Sannino, Phys. Rev. D80, 037702 (2009). [arXiv:0812.3406 [hep-ph]]. G. D. Kribs, T. S. Roy, J. Terning and K. M. Zurek, Phys. Rev. D 81, 095001 (2010) [arXiv:0909.2034 [hep-ph]]. M. R. Buckley and L. Randall, arXiv:1009.0270 [hepph]. N. Haba, S. Matsumoto and R. Sato, arXiv:1101.5679 [hep-ph]. E. J. Chun, arXiv:1102.3455 [hep-ph]. M. L. Graesser, I. M. Shoemaker and L. Vecchi, arXiv:1103.2771 [hep-ph].

[11] T. Cohen and K. M. Zurek, Phys. Rev. Lett. 104, 101301 (2010) [arXiv:0909.2035 [hep-ph]].

[12] A. Falkowski, J. T. Ruderman, T. Volansky, arXiv:1101.4936 [hep-ph].

[13] Z. Ahmed et al. [The CDMS-II Collaboration], Science 327, 1619 (2010) [arXiv:0912.3592 [astro-ph.CO]].

[14] J. Angle et al. [XENON Collaboration], Phys. Rev. Lett. 100, 021303 (2008) [arXiv:0706.0039 [astro-ph]]. 
[15] V. N. Lebedenko et al., Phys. Rev. D 80, 052010 (2009) [arXiv:0812.1150 [astro$\mathrm{ph}]]$.

[16] M. Beltran, D. Hooper, E. W. Kolb and Z. C. Krusberg, Phys. Rev. D 80, 043509 (2009) [arXiv:0808.3384 [hep-ph]].

[17] Q. H. Cao, C. R. Chen, C. S. Li and H. Zhang, arXiv:0912.4511 [hep-ph].

[18] J. Goodman, M. Ibe, A. Rajaraman, W. Shepherd, T. M. P. Tait and H. B. Yu, Phys. Lett. B 695, 185 (2011) [arXiv:1005.1286 [hep-ph]]. J. Goodman, M. Ibe, A. Rajaraman, W. Shepherd, T. M. P. Tait and H. B. Yu, Phys. Rev. D 82, 116010 (2010) [arXiv:1008.1783 [hep-ph]].

[19] Y. Bai, P. J. Fox and R. Harnik, JHEP 1012, 048 (2010) [arXiv:1005.3797 [hepph]]. P. J. Fox, R. Harnik, J. Kopp, Y. Tsai, [arXiv:1103.0240 [hep-ph]].

[20] W. H. Press and D. N. Spergel, Astrophys. J. 296, 679 (1985).

[21] A. Gould, Astrophys. J. 321, 560 (1987). A. Gould, Astrophys. J. 321, 571 (1987).

[22] K. Griest and D. Seckel, Nucl. Phys. B 283, 681 (1987) [Erratum-ibid. B 296, 1034 (1988)].

[23] D. Spolyar, K. Freese and P. Gondolo, Phys. Rev. Lett. 100, 051101 (2008) [arXiv:0705.0521 [astro-ph]].

[24] F. Iocco, A. Bressan, E. Ripamonti, R. Schneider, A. Ferrara and P. Marigo, Mon. Not. Roy. Astron. Soc. 390, 1655 (2008) [arXiv:0805.4016 [astro-ph]].

[25] M. McCullough and M. Fairbairn, Phys. Rev. D 81, 083520 (2010) [arXiv:1001.2737 [hep-ph]]. 
[26] D. Hooper, D. Spolyar, A. Vallinotto and N. Y. Gnedin, Phys. Rev. D 81, 103531 (2010) [arXiv:1002.0005 [hep-ph]].

[27] C. Kouvaris, Phys. Rev. D 77, 023006 (2008) [arXiv:0708.2362 [astro-ph]].

[28] C. Kouvaris and P. Tinyakov, Phys. Rev. D 82, 063531 (2010) [arXiv:1004.0586 [astro-ph.GA]].

[29] A. de Lavallaz and M. Fairbairn, Phys. Rev. D 81, 123521 (2010) [arXiv:1004.0629 [astro-ph.GA]].

[30] M. T. Frandsen and S. Sarkar, Phys. Rev. Lett. 105, 011301 (2010) [arXiv:1003.4505 [hep-ph]].

[31] D. T. Cumberbatch, J. A. Guzik, J. Silk, L. S. Watson and S. M. West, Phys. Rev. D 82, 103503 (2010) [arXiv:1005.5102 [astro-ph.SR]].

[32] M. Taoso, F. Iocco, G. Meynet, G. Bertone and P. Eggenberger, Phys. Rev. D 82, 083509 (2010) [arXiv:1005.5711 [astro-ph.CO]].

[33] F. Sandin and P. Ciarcelluti, Astropart. Phys. 32, 278 (2009) [arXiv:0809.2942 [astro-ph]].

[34] P. Ciarcelluti, F. Sandin, Phys. Lett. B695, 19-21 (2011). [arXiv:1005.0857 [astro-ph.HE]].

[35] L. Iorio, JCAP 1011, 046 (2010). [arXiv:1005.5078 [gr-qc]].

[36] I. Goldman and S. Nussinov, Phys. Rev. D 40, 3221 (1989).

[37] A. Gould, B. T. Draine, R. W. Romani and S. Nussinov, Phys. Lett. B 238, 337 (1990).

[38] G. Bertone and M. Fairbairn, Phys. Rev. D 77, 043515 (2008) [arXiv:0709.1485 [astro-ph]]. 
[39] C. Kouvaris and P. Tinyakov, arXiv:1012.2039 [astro-ph.HE].

[40] S. L. Shapiro, S. A. Teukolsky, New York, USA: Wiley (1983) 645 p.

[41] J. S. Clark, S. P. Goodwin, P. A. Crowther, L. Kaper, M. Fairbairn, N. Langer and C. Brocksopp, Astron. Astrophys. 392, 909 (2002) [arXiv:astro-ph/0207334].

[42] A. R. Zentner, Phys. Rev. D80, 063501 (2009). [arXiv:0907.3448 [astro-ph.HE]].

[43] J. L. Feng, M. Kaplinghat and H. B. Yu, Phys. Rev. Lett. 104, 151301 (2010) [arXiv:0911.0422 [hep-ph]].

[44] T. Lin, H. B. Yu and K. M. Zurek, arXiv:1111.0293 [hep-ph].

[45] S. O. Kepler et al., Mon. Not. Roy. Astron. Soc. 375, 1315 (2007) [arXiv:astro$\mathrm{ph} / 0612277]$.

[46] O. Kargaltsev, G. G. Pavlov and R. W. Romani, Astrophys. J. 602, 327 (2004) [arXiv:astro-ph/0310854].

[47] R. N. Manchester, G. B. Hobbs, A. Teoh and M. Hobbs, Astron. J. 129, $1993 \quad$ (2005) [arXiv:astro-ph/0412641]. http://www .atnf.csiro.au/people/pulsar/psrcat/

[48] E. H. Gudmundsson, C. J. Pethick and R. I. Epstein, Astrophys. J. 259, L19 (1982).

[49] http://www.naic.edu/ ${ }^{\sim}$ freire/GCpsr.html

[50] S. Mashchenko, A. Sills, Astrophys. J. 619, 243 (2005). [astro-ph/0409605]. S. Mashchenko, A. Sills, Astrophys. J. 619, 258 (2005). [astro-ph/0409606].

[51] C. Kouvaris, P. Tinyakov, [arXiv:1104.0382 [astro-ph.CO]]. 


\section{CHAPTER IV}

\section{The Dark Matter Inverse Problem: Extracting Particle Physics from Scattering Events}

The primary observable in dark matter direct detection is the spectrum of scattering events. We simulate multiple positive direct detection signals (on germanium, xenon, and argon targets) to explore the extent to which the underlying particle physics, manifested in the momentum dependence of the operator mediating the scattering, can be extracted. Taking into account realization (Poisson) noise, a single target nucleus with 300 events has limited power to discriminate operators with momentum dependence differing by $q^{ \pm 2}$ for a wide range of dark matter masses from $10 \mathrm{GeV}$ to $1 \mathrm{TeV}$. With the inclusion of multiple targets (or a factor of several more events on a single target), the discrimination of operators with different momentum dependence becomes very strong at the 95\% C.L. for dark matter candidates of mass $50 \mathrm{GeV}$ and above. On the other hand, operator discrimination remains poor for $10 \mathrm{GeV}$ candidates until multiple experiments each collect 1000 or more events.

Written in collaboration with Hai-Bo Yu and K. M. Zurek. Originally published as The Dark Matter Inverse Problem: Extracting Particle Physics from Scattering Events, Phys. Rev. D 85, 123507 (2012). arXiv:1110.4281 [hep-ph], MCTP-11-34. 


\subsection{Introduction}

Dark matter (DM) dominates the dynamics of matter on large scales in our universe, yet the theory which describes its underlying interactions, both with itself and with the Standard Model (SM) sector, remains hidden. Viable, testable models that are capable of both describing the DM and satisfying the observed constraints abound in the literature. The most popular DM candidate is the weakly interacting massive particle (WIMP). A well-studied example of a WIMP is the lightest supersymmetric particle in the minimal supersymmetric standard model (see e.g. [1] for a review). This candidate satisfies the observational constraints that it is long-lived, cold, and weakly interacting. Its mass is typically predicted to be between $10 \mathrm{GeV}$ and $1 \mathrm{TeV}$, and its interaction with ordinary particles is mediated by an operator that gives rise to momentum-independent interactions.

Direct detection experiments, such as XENON100 [2] and CDMS-II [3], have achieved a high level of sensitivity to a WIMP in this mass range. In general, the focus has been on ruling out DM candidates with the most standard characteristics - momentum independent scattering in the mass range well above $10 \mathrm{GeV}$. However, some recent experimental hints have led to more detailed investigations of other possibilities. In particular, the DAMA-LIBRA [4], CoGeNT [5], and CRESST-II [6] experiments have excesses which may be consistent with a DM mass of order 10 $\mathrm{GeV}$, leading to a renewed theoretical interest in DM candidates with mass in this range. However, these results are in tension with the null results of XENON10 [7,8], XENON100 [2], and CDMS [9]. This has revitalized interest in DM candidates that have non-standard features that might allow one to evade the constraints from the null experiments. In particular, operators that mediate interactions that give rise to momentum-dependent scattering rates, first considered in [10-12], have received renewed interest [13-18].

Independent of these results, direct detection experiments with increasing sensi- 
tivity are continuing to probe the WIMP DM hypothesis. The XENON collaboration will soon begin construction on a XENON1T phase [19]; a 3.6 ton liquid argon detector operated by the DEAP/CLEAN collaboration, DEAP-3600 [20], is under construction; and $100 \mathrm{~kg}$ and 1 ton cryogenic germanium detectors are being planned [21] by the Super-CDMS/GEODM collaborations. If there is a positive signal from one of these experiments, the signal must be confirmed by multiple experiments with different targets. Furthermore, one would like to determine experimentally whether the scattering is in fact mediated by a standard spin-independent operator or whether the underlying particle physics is more complex, with non-standard types of momentum dependence in the scattering. Additionally, recent theoretical work has focused on the feasibility of extracting different types of information from scattering events [22, 23].

In this paper we explore the dark matter inverse problem; that is, the capability of direct detection experiments to extract the underlying particle physics mediating the scattering of a DM particle. In practice this question boils down to how well the momentum dependence in an operator can be mimicked by other operators for a given target and DM mass. We simulate detection events using different types of interactions with varying momentum and velocity dependence, then we fit these events by a wide variety of interactions. In principle, one can distinguish interaction types by just checking the recoil spectra for an individual target. For example, if DM scatters with the target nucleus through an interaction with a positive momentum dependence, one would expect events to drop in low recoil energy bins, while the event rate increases exponentially towards low recoil energy for a standard momentum independent interaction. However, if only one target is available, the capability for distinguishing operators through the spectra in this way is limited by the experimental noise and detector threshold. With multiple nuclear targets one may also compare the overlap of the DM preferred regions on different target nuclei to determine the correct operator. We find that, for similar cross-sections, analysis of high mass DM particles leaves 
distinctive qualitative imprints that allow one to extract the momentum dependence in the scattering. When two operators cannot be distinguished between each other, it is because they have a very similar momentum dependence. On the other hand, operator discrimination for a $10 \mathrm{GeV}$ candidate is poor. We conclude that a significantly lower threshold, or much improved statistics, than is available for the next generation of experiments will be necessary in order to extract the particle physics mediating the scattering for a light DM candidate.

We begin the paper by briefly reviewing direct detection basics. In Section III we discuss our methodology, including detector effects, analysis methods, and conventions. In Section IV we go over our results. Finally, we summarize our conclusions in Section V.

\subsection{Preliminaries}

We compare the spectra for standard spin- and momentum-independent scattering against operators with momentum dependence. We call operators with $n$ additional powers of momentum transfer $q^{n}$-type scattering, where $n= \pm 2, \pm 4$. We also consider scattering via anapole and dipole operators, which feature mixed momentum

dependence (constant and $q^{2}$ dependence for the former operator, and $q^{2}$ and $q^{4}$ dependence for the latter). In this section we review the rate relations for the various types of operators and define our conventions for reporting results.

For generic scattering the observed differential rate of observation of DM particles may be written

$$
\frac{d R}{d E_{R}}\left(E_{R}\right)=\frac{\rho_{0}}{m_{\mathrm{DM}} m_{N}} \int d^{3} \vec{v} v f\left(v_{0}, \vec{v}_{e} ; v_{m i n}, v_{e s c}\right) \epsilon_{\mathrm{eff}}\left(E_{R}\right) \frac{d \sigma_{\mathrm{DM}-N}}{d E_{R}}\left(v, A, E_{R}\right)
$$

Here, $\rho_{0}$ is the local DM density; $m_{\mathrm{DM}}$ is the DM mass; $m_{N}$ is the detector nucleus mass; the velocity distribution is given by $f\left(v_{0}, \vec{v}_{e} ; v_{m i n}, v_{e s c}\right) ; \epsilon_{\mathrm{eff}}\left(E_{R}\right)$ is the detector 
efficiency, which may depend on the energy of recoil; and the differential cross-section $d \sigma_{\mathrm{DM}-N} / d E_{R}$ describes the interaction. The total number of events is given by integrating the differential rate as

$$
N_{i}\left(E_{R}^{\min }, E_{R}^{\max }\right)=\int_{E_{R}^{\min , i}}^{E_{R}^{\max , i}} d E_{R} \frac{d R}{d E_{R}} \epsilon_{\exp },
$$

where $\epsilon_{\exp }$ is the exposure, generally given in terms of kilograms of exposed material multiplied by the days of duration of the experiment.

All of the particle physics information present in Eq. (4.1) is contained in the final term, $d \sigma_{\mathrm{DM}-N} / d E_{R}$, while the astrophysical considerations are reflected in $\rho_{0}$ and $f\left(v_{0}, \vec{v}_{e} ; v_{m i n}, v_{e s c}\right)$. The remaining effects, such as $\epsilon_{\text {eff }}\left(E_{R}\right), \epsilon_{\exp }$, and $m_{N}$, are detectorspecific. The objective of this paper is to extract information about the operator content of $d \sigma_{\mathrm{DM}-N} / d E_{R}$ based solely on observables. We fix the astrophysical parameters by the best current observations and assume the detector effects are well understood. In particular, we take $\rho_{0}=0.4 \mathrm{GeV} / \mathrm{cm}^{3}$ and assume a Maxwell-Boltzmann velocity distribution with a smooth exponential cutoff at the escape velocity:

$$
f\left(v_{0}, \vec{v}_{e} ; v_{m i n}, v_{e s c}\right)=\left\{\begin{array}{ll}
\frac{1}{N}\left(e^{-\left(\vec{v}_{e}+\vec{v}_{\mathrm{DM}}\right) \cdot\left(\vec{v}_{e}+\overrightarrow{\mathrm{v}}_{\mathrm{DM}}\right) / v_{0}^{2}}-e^{-v_{e s c}^{2} / v_{0}^{2}}\right) & v<v_{e s c} \\
0 & v>v_{e s c}
\end{array},\right.
$$

where we take $v_{\text {esc }}=544 \mathrm{~km} / \mathrm{s}, v_{0}=220 \mathrm{~km} / \mathrm{s}$, and $v_{e}=232 \mathrm{~km} / \mathrm{s}$. The normalization $N$ for this distribution can be found in the appendix of [16]. We find that our results are insensitive to changes in these velocity parameters. Note here that we are only interested in extracting the correct particle physics interaction. This differs from recent work where the goal was to marginalize over astrophysical uncertainties while extracting the basic physical parameters [23]. Astrophysical uncertainty will enlarge the preferred region in the relevant parameter space for a single experiment, but our results involve a comparison of multiple experiments for which the astrophysics are 
the same. We have checked that our results are unchanged as long as we use the same inputs for the different experiments and do not marginalize over the unknown quantities.

The differential scattering cross-section is related to the DM-nucleus scattering cross-section $\sigma_{N}$ via

$$
\frac{d \sigma_{\mathrm{DM}-N}}{d E_{R}}=\frac{m_{N} \sigma_{N}}{2 \mu_{N}^{2} v^{2}}
$$

where $\mu_{N}$ is the reduced mass of the DM-nucleus pair and $v$ is their relative velocity. For standard spin- and momentum-independent scattering, which we denote "std", the results are quoted in terms of the cross-section for scattering off a nucleon, $\sigma_{n}^{\text {std }}$ :

$$
\sigma_{N}^{s t d}=\sigma_{n}^{s t d} \frac{\mu_{N}^{2}}{\mu_{n}^{2}} \frac{\left[f_{p} Z+f_{n}(A-Z)\right]^{2}}{f_{p}^{2}} F_{1}^{2}\left(A, E_{R}\right),
$$

where $\mu_{n}$ is the reduced mass of the DM-nucleon pair. We take $f_{p}=f_{n}=1$ here, and assume a standard Helm form factor, $F_{1}\left(A, E_{R}\right)=3 j_{1}\left(q r_{0}\right) /\left(q r_{0}\right) e^{-(q s)^{2} \mathrm{fm}^{2} / 2}$, with $s=0.9$ and $r_{0}=\left(\left(1.23 A^{1 / 3}-0.6\right)^{2}+7 / 3(0.52 \pi)^{2}-5 s^{2}\right)^{1 / 2} \mathrm{fm}[25]$.

For spin-independent, momentum-dependent scattering, the results will be reported according to the convention [13]:

$$
\sigma_{N}^{q^{n}}=\left(\frac{q}{q_{0}}\right)^{n} \sigma_{N}^{s t d}
$$

In this work, we take $q_{0}=50 \mathrm{MeV}$ for all values of $n^{1}$. We allow $\sigma_{n}$ to float for both the standard and $q^{n}$ type operators to satisfy normalization conditions described below. In general, this will lead to $\sigma_{n} \sim \mathcal{O}\left(10^{-45}\right) \mathrm{cm}^{2}$. Note that the momentumtransfer dependence of the $q^{n}$-type operators serves to introduce extra powers of the recoil energy, not extra powers of the DM velocity.

\footnotetext{
${ }^{1}$ Particle physics realizations of momentum-dependent scattering cross sections are discussed in [13]. Note that [13] assumes that the mediator mass is heavier than the momentum transfer so that $n=2,4$ are positive. If the mediator mass is smaller than the momentum transfer, we can have $n=-2,-4$.
} 
Lastly, we define our conventions for operators with mixed momentum dependence, the anapole $\left(\bar{\chi} \gamma^{\mu} \gamma_{5} \chi A_{\mu}\right)$ and dipole $\left(\bar{\chi} \sigma^{\mu \nu} \chi F_{\mu \nu}\right)$ operators. The scattering cross-sections for these operators are [16]

$$
\begin{aligned}
\sigma_{N}^{a n}= & \frac{\mu_{N}^{2}}{4 \pi M^{4}}\left\{4 v^{2} Z^{2} F_{1}^{2}\left(A, E_{R}\right)-q^{2}\left[\frac{1}{\mu_{N}^{2}} Z^{2} F_{1}^{2}\left(A, E_{R}\right)-2 A^{2} \frac{J+1}{3 J} \frac{b_{N}^{2}}{m_{N}^{2} b_{n}^{2}} F_{2}^{2}\left(A, E_{R}\right)\right]\right\}, \\
\sigma_{N}^{d i p}= & \frac{4 \mu_{N}^{2}}{\pi M^{4} \Lambda^{2}}\left\{4 q^{2} v^{2} Z^{2} F_{1}^{2}\left(A, E_{R}\right)\right. \\
& \left.\quad-q^{4}\left[\left(\frac{2}{m_{\mathrm{DM}} m_{N}}+\frac{1}{m_{N}^{2}}\right) Z^{2} F_{1}^{2}\left(A, E_{R}\right)-2 A^{2} \frac{J+1}{3 J} \frac{b_{N}^{2}}{m_{N}^{2} b_{n}^{2}} F_{2}^{2}\left(A, E_{R}\right)\right]\right\} .
\end{aligned}
$$

Here, $M$ is the mass of a mediator particle that couples an off-shell photon to the DM particle, while $\Lambda$ is a confinement or compositeness scale that describes the magnetic dipole moment physics; we take it to be $100 \mathrm{MeV}$ in all of our analysis. The mediator mass $M$ is a priori unknown. In general, it floats around a fiducial value of a few hundred GeV. $J$ is the spin of the target nucleus, $b_{N}$ is the magnetic moment of the target nucleus, and $b_{n}=e / 2 m_{p}$ is the Bohr magneton. The form factor $F_{2}\left(A, E_{R}\right)$ for the spin coupling term is important for spin-odd nuclei and is taken from [26]. Since the isoscalar and isovector couplings are model-dependent, we choose to take $a_{p}=a_{n}$ in this work. We find that the results are qualitatively insensitive to these exact values.

The normalized interaction strength that we display on the plots for both anapole and dipole moment operators is

$$
\widehat{\sigma}_{n}=\frac{\mu_{n}^{2}}{4 \pi M^{4}} \simeq 1.05 \times 10^{-39}\left(\frac{\mu_{n}}{m_{n}}\right)^{2}\left(\frac{M}{400 \mathrm{GeV}}\right)^{-4} \mathrm{~cm}^{2} .
$$

The fiducial value of $M$ and thus of $\widehat{\sigma}_{n}$ differs depending on the type of interaction and the mass of the DM, but these values are chosen to obey the same normalization conditions alluded to above. Our normalization conventions are described in greater 
detail in the next section.

\subsection{Methodology}

We simulate mock experimental ("input") data, and compare the goodness-offit of theoretical ("fit") spectra against the input data. Ideal experimental data is first generated via a model, then smeared according to both Poisson statistics and the finite energy resolution of the experiment. We then fit theoretical models (convoluted to take into account detector resolution) to the input data using a log-likelihood ratio test. We describe our methodology in this section.

\subsubsection{Detector and Statistical Effects}

In order to fully simulate a direct detection experiment, one must fold detector effects into the rate given in Eq. (4.1). A convolution integral (accounting for the finite energy resolution of the detector) and Poisson noise (expected in a counting type experiment) are the most important of these effects. For low mass DM in particular, these considerations are equally important in drawing conclusions. We describe here how we take these effects into account.

Convolution. In order to compare a theoretical model against an input data set, the rates must be smeared to take into account detector effects and noise. Both the input data and the theoretical spectra are smeared according to the energy resolution, because a realistic detector does not have perfect energy resolution. The observed

rate is well modeled by convolving the expected rate with an approximately Gaussian distribution whose width is a function of detector element and true recoil energy:

$$
\frac{d \widetilde{R}}{d E_{R}}=\int d E^{\prime} \frac{d R}{d E_{R}}\left(E^{\prime}\right) \frac{1}{\sqrt{2 \pi} \sigma_{d e t}\left(A, E^{\prime}\right)} \exp \left[-\frac{\left(E_{R}-E^{\prime}\right)^{2}}{2 \sigma_{d e t}^{2}\left(A, E^{\prime}\right)}\right]
$$




\begin{tabular}{|l|r|r|r|l|r|}
\hline Element & $A$ & $Z$ & $J$ & $b_{N} / b_{n}$ & Abundance \\
\hline Germanium & 73 & 32 & $9 / 2^{+}$ & -0.879 & $7.73 \%$ \\
\hline Xenon & 129 & 54 & $1 / 2^{+}$ & -0.778 & $26.4 \%$ \\
\hline Xenon & 131 & 54 & $3 / 2^{+}$ & +0.692 & $21.2 \%$ \\
\hline
\end{tabular}

Table 4.1: Physical properties of stable spin-odd nuclei present in detectors. The dominant argon nucleus has $A=40$, and there are no spin-odd isotopes with appreciable abundance.

For all isotopes present in a given detector we define the detector energy resolutions to be

$$
\begin{aligned}
& \sigma_{\text {det }}(\mathrm{Ge}, E)=\sqrt{(0.3)^{2}+(0.06)^{2} E / \mathrm{keV}} \mathrm{keV}, \\
& \sigma_{\text {det }}(\mathrm{Xe}, E)=0.6 \sqrt{E / \mathrm{keV}} \mathrm{keV}, \\
& \sigma_{\text {det }}(\mathrm{Ar}, E)=0.7 \sqrt{E / \mathrm{keV}} \mathrm{keV} .
\end{aligned}
$$

In all cases we take a flat efficiency. The isotopic abundances are crucial for extracting information in the anapole and dipole cases. We list the spins, magnetic moments, and abundances of the important spin-odd nuclei for each element in Table 4.1, and take the abundances for the spin-even nuclei to be set by naturally occurring levels.

Integrating the convoluted rate in Eq. (4.9) over the recoil energy range in each bin $i$ gives rise to binned observation numbers

$$
\widetilde{N}_{i}\left(E_{R}^{\min }, E_{R}^{\max }\right)=\int_{E_{R}^{\min , i}}^{E_{R}^{\max , i}} d E_{R} \frac{d \widetilde{R}}{d E_{R}} \epsilon_{\text {exp }}
$$

These binned observation numbers produce the observable quantities that make up both our input data and the data used in our fits. We will refer to the vector of data points generated by Eq. (4.13) as the "convoluted spectrum."

Poisson Noise. We expect that our input data are one of an ensemble of many other data that could be produced by the expected model. Since the input data that 
come from any given experiment are produced by counting, we expect that the input data will deviate from the convoluted spectra given in Eq. (4.13) at a level determined by Poisson statistics. To simulate the observed events, we thus introduce stochastic Poisson noise distributed about the spectrum given in Eq. (4.13).

We have checked that, as expected, the average CLC generated by many noisy data sets converges on a CLC described by data generated by inputs from Eq. (4.13) with no noise.

Log-Likelihood Ratio Test. We use a log-likelihood ratio to provide an estimate of the fit parameters that best reproduce a set of input data. For input data $n_{i}$ in the $i$-th energy bin (which has been smeared in energy as described above and in the number of events according to Poisson statistics) and theoretical parameters $\theta$ that produce a convoluted spectrum with values $\nu_{i}$, the $\log$-likelihood ratio is

$$
\widetilde{L}=2 \sum_{i=1}^{N}\left[\nu_{i}(\theta)-n_{i}+n_{i} \ln \frac{n_{i}}{\nu_{i}(\theta)}\right]+\text { const. }
$$

where if $n_{i}=0$ the last term in the brackets is set to zero. ${ }^{2}$ The parameters of interest are the DM-nucleon cross section and DM mass. The parameters that minimize Eq. (4.14) provide the point of best fit, and contours of constant $\widetilde{L}$ values give confidence level regions. To define the $95 \%$ confidence level regions we take the region inside of which $\widetilde{L} \leq \widetilde{L}_{\min }+\Delta \widetilde{L}_{95}^{2}$ d.o.f., where for instance $\Delta \widetilde{L}_{95}^{2}$ d.o.f. $=5.99$ [38].

\subsubsection{Defining the Spectra}

We generate a vector of input data in the $i$ energy bins, $n_{i}$, for all three target nuclei assuming interactions derived from standard, anapole, dipole, $q^{2}$, and $q^{-4}$ operators. Recall that the input data are smeared in energy as well as with Poisson noise. These

\footnotetext{
${ }^{2}$ The value of $\nu_{i}$ should never be exactly zero because even at high energy the convolution integral will receive some contribution from events in the low energy tail of the convolution.
} 
spectra are obtained for DM masses of 10,50 , and $250 \mathrm{GeV}$. This is then compared to the vector of theoretical data in the $i$ energy bins, $\nu_{i}$, which are the convoluted spectra for standard, anapole, dipole, $q^{ \pm 2}$, and $q^{ \pm 4}$ operators.

For xenon and germanium experiments, we take the energy range of the experiment to be $5-60 \mathrm{keV}$, binned in a $1 \mathrm{keV}$ interval from 5 to $13 \mathrm{keV}, 2 \mathrm{keV}$ from 13 to $25 \mathrm{keV}$, and $5 \mathrm{keV}$ from 25 to $60 \mathrm{keV}$. For the argon experiment, a higher threshold is assumed, taking the energy range of the experiment to be $20-60 \mathrm{keV}$, binned in 1 $\mathrm{keV}$ from 20 to $28 \mathrm{keV}, 2 \mathrm{keV}$ from 28 to $40 \mathrm{keV}$, and $5 \mathrm{keV}$ from 40 to $60 \mathrm{keV}$. These bins are moderately coarser than current and upcoming experimental capabilities, but we find that the results are insensitive to bin size. We have checked that more than doubling the number of allowed bins and using bins as narrow as $0.4 \mathrm{keV}$ does not affect our results. In all cases, properly accounting for the effect of the noise and the energy resolution are more important than binning choices for reaching robust conclusions.

Our conventions for the total rate may be fixed in two ways. First, we take the exposure of all targets to be 2 ton-years and fix the total number of events on germanium to be 300 for 50 and $250 \mathrm{GeV}$ DM candidates and 100 for a $10 \mathrm{GeV} \mathrm{DM}$ candidate. We allow $\sigma_{n}$ and $\widehat{\sigma}_{n}$ to vary so as to achieve the desired number of events on germanium. For a fixed exposure more events are observed on a xenon target due to the higher atomic number, while fewer are observed on an argon target. To check the robustness of our results and their sensitivity to statistics, we also calculate results when an equal number of events are obtained on all targets (300 events for 50 and $250 \mathrm{GeV}$ DM test masses, and 100 events for $10 \mathrm{GeV}$ DM test mass). This will allow us to disentangle the effect of statistics from the effects of particle physics. We find that while the constraining power of individual experiments changes, we are able to obtain good discrimination in both cases.

Due to threshold effects, fewer events are expected to result for $10 \mathrm{GeV}$ DM 
candidates for a given scattering cross-section. As a result of these fewer events, noise is an important limiting factor in our ability to definitively extract the correct operator mediating the interaction. For light DM, kinematics is also very limiting. For these reasons, our conclusions for light DM are less sensitive to the total number of events: even with as many as 1000 events on a single target (which for a germanium target corresponds to 20 ton-years of exposure for a cross-section $\sigma_{n} \sim 10^{-44} \mathrm{~cm}^{2}$ ) we do not obtain good discrimination for a $10 \mathrm{GeV}$ candidate.

\subsection{Results}

There are two possible measures for operator discrimination that will be obvious from our results: the absolute goodness-of-fit per degree of freedom, $\widetilde{L}_{\min } /$ d.o.f., and the overlap of the $95 \%$ preferred regions from fits to spectra generated by different target nuclei. A mismatch of the input data and theoretical model should give high log-likelihood values, and comparing the $\widetilde{L}_{\text {min }} /$ d.o.f. values for different trial operators can provide a clue to nature of the interaction that generated the data. Similarly, different operators will give rise to different preferred regions as observed on multiple targets. Overlapping confidence level curves (CLCs) can indicate that the momentum dependence scales with target nucleus as expected for the hypothesized interaction and disjoint CLCs can indicate the converse. As detailed below, considering the value of $\widetilde{L}_{\text {min }}$ for the combined data from all targets gives a test that is sensitive to both of these measures.

Two subsets of the results are shown in Figs. (4.1) and (4.2) for input data corresponding to standard and dipole mediated scattering. More complete results are available in the appendix and online [27]. The subsets shown in Figs. (4.1) and (4.2) are chosen to represent illustrative contrasts to the input data; we describe our particular choices in more detail below. Each figure corresponds to one set of input data (labeled by $n_{i}$ ) generated according to the methodology described in the previous 
section. The individual panels of each figure correspond to fits of a theoretical model (labeled by $\nu_{i}$ ) to the input data. We divide the plots in Figs. (4.1) and (4.2) so that the left panels show our results for the 10 and $50 \mathrm{GeV}$ DM candidates for scattering on all three targets: xenon, germanium and argon. ${ }^{3}$ The right panels show the same information for $250 \mathrm{GeV}$ candidates. In the appendix, all CLCs are shown on the same plot. The colors on the curves correspond to the minimum log-likelihood per degree of freedom ${ }^{4}$ for the fit of each operator to the data, with darker colors representing the better fits. We have checked that different realizations of noise do not change our ability to extract information about the particle physics.

In this section, we will start by going through a simplified analysis of the scattering kinematics that explains the shapes of the CLCs in Figs. (4.1) and (4.2). Next, we will explain how the tests discussed here allow one to extract the correct particle physics that underlies direct detection events. We will do this both when all exposures are the same and when the number of observed events is the same on all targets. This allows us to determine the effects of statistics on our results. Finally, we will compare models using the p-value test. This test will be a quantitative measure of both of the qualitative tests described above.

\subsubsection{Contour Shapes from Scattering Kinematics}

We discuss the shapes of the CLCs that we observe in Figs. (4.1) and (4.2). The kinematics of elastic scattering between DM particles and detector target nucleus provides a straightforward handle on the shape of these preferred regions. For the purpose of illustration, we take standard scattering and simplify the differential event

\footnotetext{
${ }^{3}$ We omit the argon curves in the $10 \mathrm{GeV}$ case because argon events generated for the $10 \mathrm{GeV}$ analysis were essentially compatible with zero. Our argon curves are therefore closer to exclusion curves than preferred regions. In all cases the germanium and xenon CLCs fit safely underneath the argon "exclusion curve."

${ }^{4}$ For high mass, d.o.f. $(\mathrm{Ge}, \mathrm{Xe})=19$, while d.o.f. $(\mathrm{Ar})=16$, corresponding to the number of $E_{R}$ bins minus the two fit parameters, $m_{D M}$ and $\sigma_{n}$. For low mass candidates we have many fewer degrees of freedom because fewer bins are filled: d.o.f.(Ge) $=6$ and d.o.f.(Xe) $=1-5$, depending on the operator.
} 
rate given in Eq. (4.1) with the limit $v_{e s c} \rightarrow \infty$, ignoring solar motion, terrestrial motion, detector efficiency, and detector resolution. This recovers the result [1]

$$
\frac{d R}{d E_{R}}\left(E_{R}\right) \simeq \frac{\rho_{0} \sigma_{N}}{\sqrt{\pi} m_{\mathrm{DM}} \mu_{N}^{2} v_{0}} \exp \left(-\frac{E_{R} m_{N}}{2 \mu_{N}^{2} v_{0}^{2}}\right)
$$

The balance between the exponential term and the linear term on the right-hand side of Eq. (4.15) gives most of the important kinematic information. This balance is the key to understanding the basic content of our plots. To better understand the implications of scattering kinematics on the operator discrimination, we consider two extreme limits first.

If the DM mass is much less the target nucleus mass then $\mu_{N} \sim m_{\mathrm{DM}}$ and we can approximate the differential rate by

$$
\frac{d R}{d E_{R}}\left(E_{R}\right) \sim \frac{\rho_{0} \sigma_{N}}{\sqrt{\pi} m_{\mathrm{DM}}^{3} v_{0}} \exp \left(-\frac{E_{R} m_{N}}{2 m_{\mathrm{DM}}^{2} v_{0}^{2}}\right) .
$$

We can immediately see that this rate is very sensitive to the DM mass. If we increase the DM mass the scattering rate will increase sharply because of the exponential term. Our numerical results clearly reflect this sensitive dependence. From fits of the 10 GeV case shown in Figs. (4.1) and (4.2) we can see that all the $10 \mathrm{GeV}$ contours are very narrow. By contrast, the event rate is linearly proportional to the scattering cross section and the shift has a rather mild dependence on the target mass. Hence, detectors have poor cross-section discrimination capabilities for the low mass case.

At the other end of the mass range, if the DM is much heavier than the detector nucleus mass then the differential event rate is given to first order by

$$
\frac{d R}{d E_{R}}\left(E_{R}\right) \sim \frac{\rho_{0} \sigma_{N}}{\sqrt{\pi} m_{\mathrm{DM}} m_{N}^{2} v_{0}} \exp \left(-\frac{E_{R}}{2 m_{N} v_{0}^{2}}\right) .
$$

The exponential term is independent of the DM mass in this limit, and it becomes 
an overall factor for any given large DM mass. The differential event rate now just scales as $d R / d E_{R} \propto \sigma_{n} / m_{\mathrm{DM}}$. In this limit the target nucleus is not able to determine the DM mass because one always can rescale $\sigma_{n}$ to compensate the change of DM mass while keeping $\sigma_{n} / m_{\mathrm{DM}}$ unchanged. This so-called high-mass degeneracy [23] is observed in Figs. (4.1) and (4.2). The allowed mass range can be unbounded for all three targets when high-mass degeneracy is important.

These features explain certain coarse aspects of the CLC plots. Now we examine the physics that leads to the separation of the CLCs in the parameter space, and we investigate what this can tell us about the particle physics underlying the data. We do this analysis first with equal exposures on all targets. We will also present results with the same number of events recorded by all targets.

\subsubsection{Operator Discrimination with Equal Exposures on All Targets}



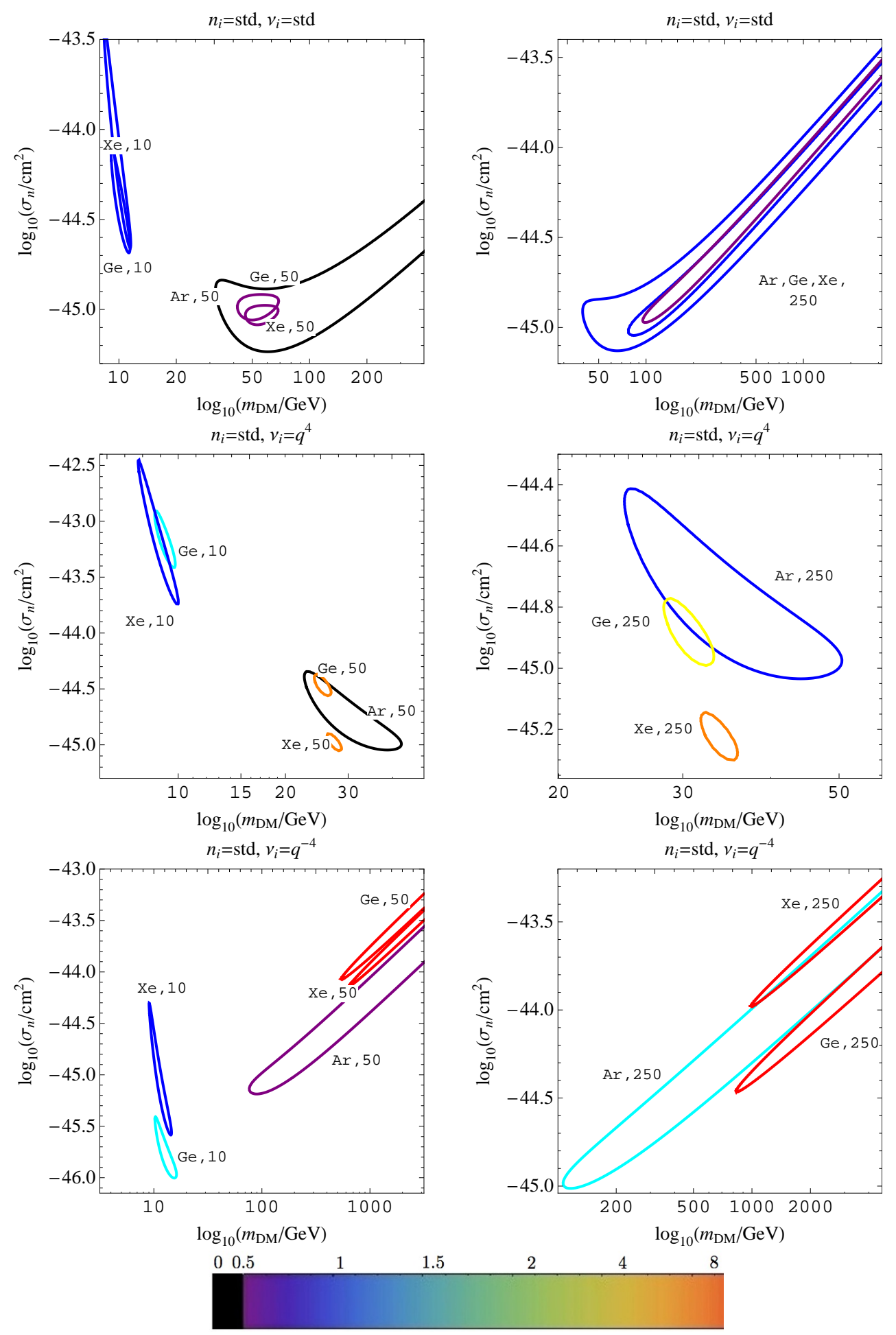

Figure 4.1: 95\% CLCs for a 10, 50, and $250 \mathrm{GeV}$ particle interacting through an $n_{i}=$ standard operator with equal exposures, such that there are 300 events on a germanium target and more (fewer) on a xenon (argon) target. Comparisons are made to $\nu_{i}=$ standard, $q^{4}$, and $q^{-4}$ operators. The colors represent the value of $\widetilde{L}_{\text {min }} /$ d.o.f. The standard interaction can be distinguished from the $q^{ \pm 4}$ operators via both CLC overlap and the values of $\widetilde{L}_{\text {min }} /$ d.o.f. As can be seen in Fig. (10) in the appendix, $\widetilde{L}_{\min } /$ d.o.f. is less powerful for distinguishing $n_{i}=$ standard from $\nu_{i}=q^{ \pm 2}$, though overlap remains robust. 

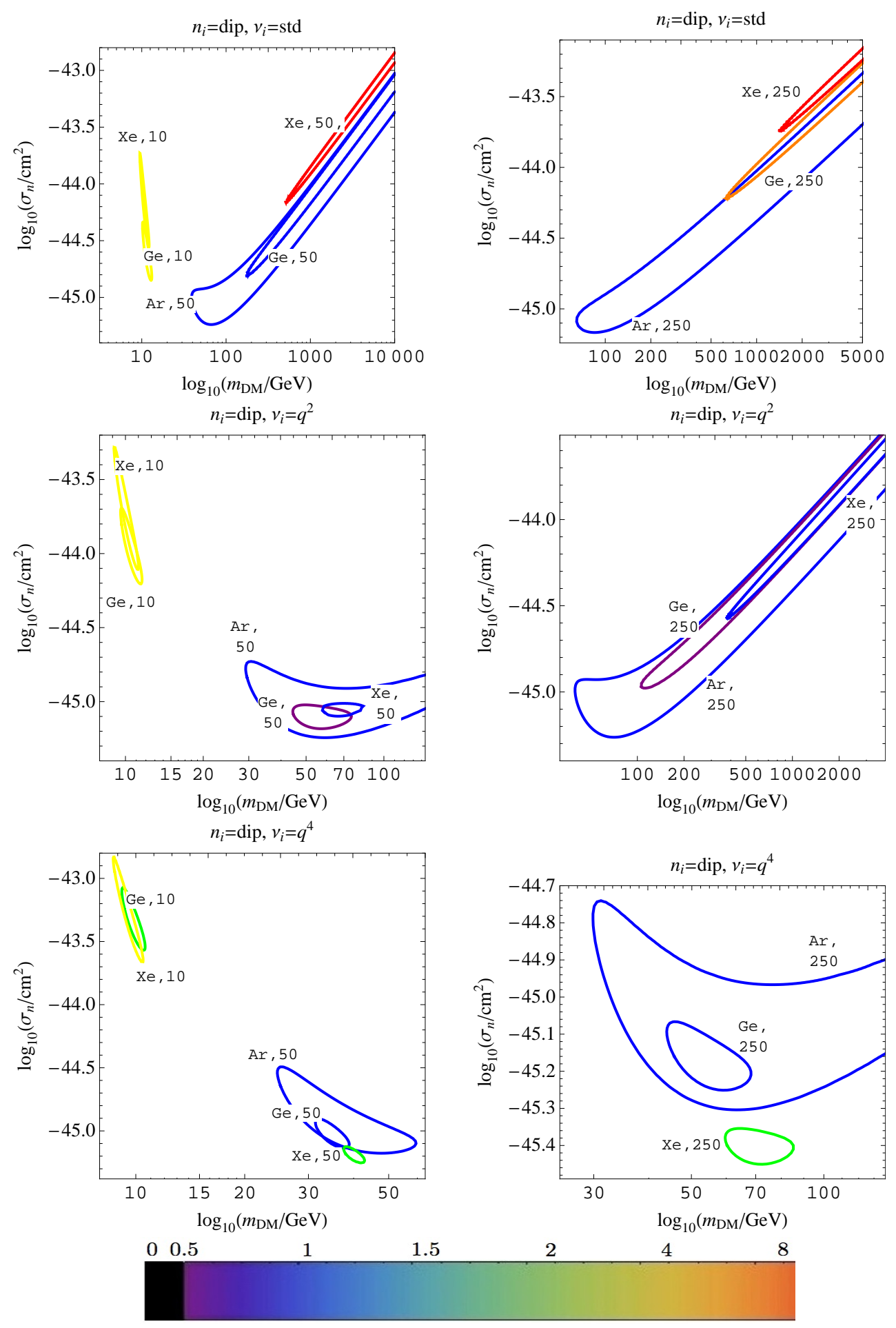

Figure 4.2: As in Fig. 4.1, but with an $n_{i}=$ dipole operator compared to $\nu_{i}=$ standard, $q^{2}$, and $q^{4}$ operators. 
For operator discrimination with equal exposures, the most important feature of Figs. (4.1) and (4.2) is that, when the input data and theoretical model match $\left(n_{i}=\nu_{i}\right)$, the CLCs overlap regardless of candidate mass. When the theoretical model is mismatched with the input data, the $50 \mathrm{GeV}$ and $250 \mathrm{GeV}$ CLCs separate, though much less separation is apparent in the $10 \mathrm{GeV}$ case. Thus for low mass candidates the overlap test does not strongly discriminate between models, while for high mass candidates the overlap test allows strong discrimination. Much of this power is derived from the strong statistics gained from scattering off of a xenon target. By contrast, we see in Fig. (4.2), where the momentum dependence of the operators differs by $q^{2}$, that, for scattering off a single target nucleus, the log-likelihood per degree-offreedom test can be limited by noise and does not provide a very good discriminant between theoretical models - the fit can be just as good on a single target with the incorrect theoretical model as it is with the correct theoretical model, even for high mass candidates. This is especially true for argon, which has the fewest number of events and smallest energy range. Xenon, however, has sufficient statistics to be able to extract the correct operator mediating the scattering on its own. The good discrimination of xenon is what makes the overlap between the xenon, germanium, and argon CLCs a sensitive test.

We now investigate how CLC separation occurs. In Fig. (4.1) we compare input data corresponding to standard momentum independent scattering to scattering from $q^{ \pm 4}$ interactions. We specifically choose $q^{ \pm 4}$ because these operators have the most exaggerated kinematic effects. These comparisons will lead to the largest separations in parameter space and show the widest disparity in the values of $\widetilde{L}_{\text {min }} /$ d.o.f. The differential event rate of the mismatched operators is given by

$$
\frac{d R^{q^{ \pm 4}}}{d E_{R}}\left(E_{R}\right) \simeq \frac{A^{2} \rho_{0} \sigma_{n}^{s t d}}{\sqrt{\pi} m_{\mathrm{DM}} \mu_{N}^{2} v_{0}}\left(\frac{\sqrt{2 E_{R} m_{N}}}{q_{0}}\right)^{ \pm 4} \exp \left(-\frac{E_{R} m_{N}}{2 \mu_{N}^{2} v_{0}^{2}}\right)
$$



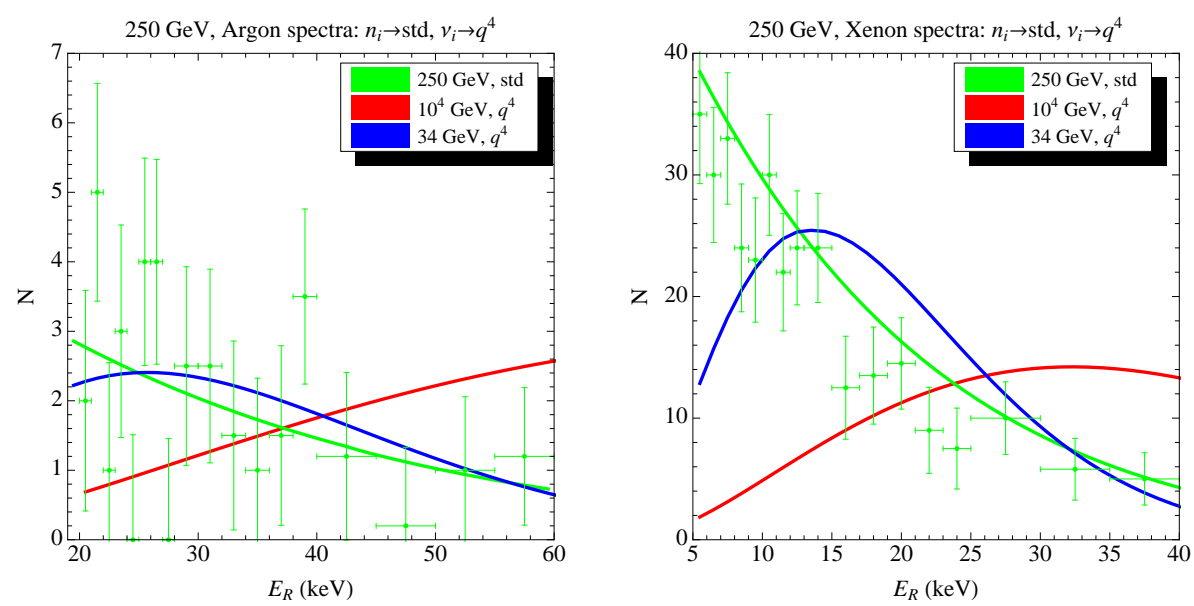

Figure 4.3: Effect of momentum dependence and DM mass on fit spectra. The data are from an $n_{i}=$ standard interaction and are depicted with Poisson error bars. The best fit spectrum for $\nu_{i}=$ standard is shown as a green solid line. We also show two $\nu_{i}=q^{4}$ spectra. The $q^{4}$ spectrum in blue is at the best fit point and the $q^{4}$ spectrum in red is taken at high mass to illustrate the improvement in goodness of fit from reducing $m_{\mathrm{DM}}$. We can also see the effect of xenon's higher event rate and lower energy threshold on its ability to determine the correct operator.

The most noticeable effect of the additional momentum dependence, as shown in the second and third rows of panels of Fig. (4.1), is that on all targets the incorrect operators prefer different DM mass ranges than the value chosen by the correct operator: $q^{4}$ scattering chooses low mass and $q^{-4}$ scattering prefers high mass.

This behavior is driven by the shape of the event distributions. As seen in Fig. (4.3), the $q^{+n}$ operator with a lower mass candidate can mimic the scattering of a higher mass candidate with a standard spectrum. This occurs because as one decreases $m_{\mathrm{DM}}$ the event number increases at low energy and decreases at high energy. Likewise, we can consider a $q^{-4}$ operator, shown in the bottom row of Fig. (4.1). In this case the event rate is suppressed at high recoil but increased at low recoil. In Fig. (4.4) we see that larger DM masses are necessary to suppress the divergent low energy tail of the $q^{-4}$ operator.

Changing the momentum dependence by only two powers, e.g. comparing $n_{i}=$ 

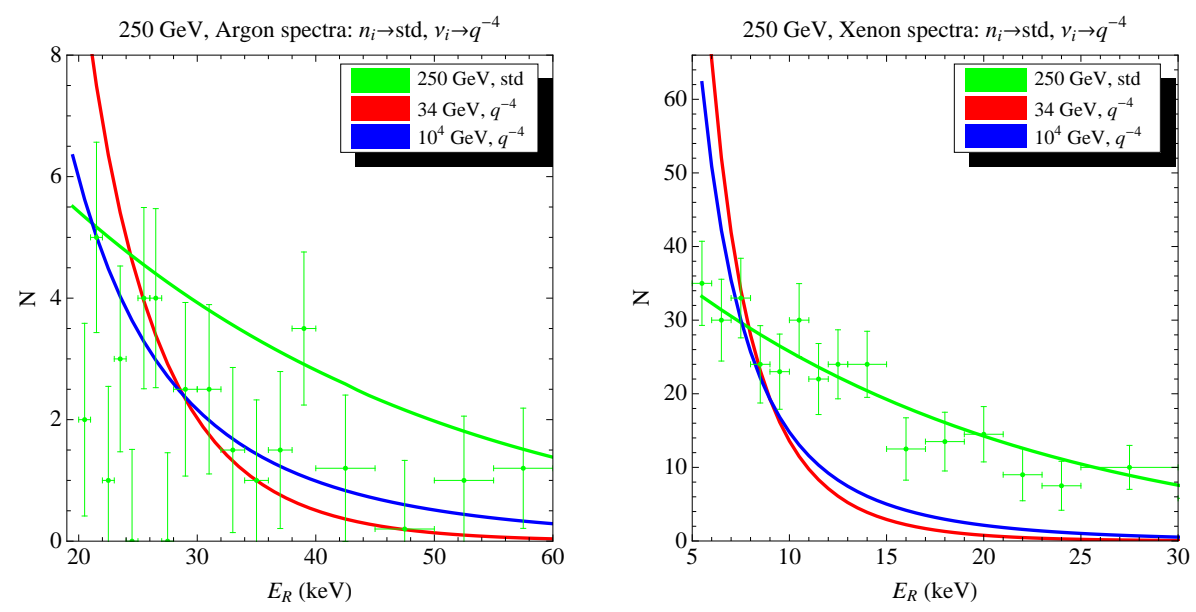

Figure 4.4: Effect of momentum dependence and DM mass on fit spectra. The data are from an $n_{i}=$ standard interaction and are depicted with Poisson error bars. The best fit spectrum for $\nu_{i}=$ standard is shown as a green solid line. We also show two $\nu_{i}=q^{-4}$ spectra. The $q^{-4}$ spectrum in blue is at the best fit point and the $q^{-4}$ spectrum in red is taken at low mass to illustrate the improvement in goodness of fit from increasing $m_{\mathrm{DM}}$. We can also see the effect of xenon's higher event rate and lower energy threshold on its ability to determine the correct operator.

std with $\nu_{i}=q^{ \pm 2}$, offers less contrast. In this case, fits for individual elements can in general be acceptable (with $\widetilde{L}_{\text {min }} /$ d.o.f. $\lesssim 1$ ), and the overlap test seems to be an important discriminant. This can be seen for the $n_{i}=$ dipole case displayed in Fig. (4.2), where we compare the dipole input data to standard, $q^{2}$, and $q^{4}$ interactions. We see that discrimination is marginal for an individual target (since $\widetilde{L}_{\text {min }} /$ d.o.f can be good), but the overlap appears to provide conclusive discrimination against the standard and $q^{4}$-type interactions. By contrast, $q^{2}$ and dipole interactions look nearly identical. This is because the $q^{2}$ term in Eq. (4.7) dominates the $q^{4}$ term for the elements and DM masses examined here, as seen in Fig. (4.5). As expected, the standard spectra are discrepant with the dipole spectra due to a divergent low mass tail, the $q^{4}$ operator suffers from being overly suppressed at low energy, and the $q^{2}$ operator provides very good fits. For the same reason, we see in the appendix that there is a degeneracy between the standard and anapole operators. Although 

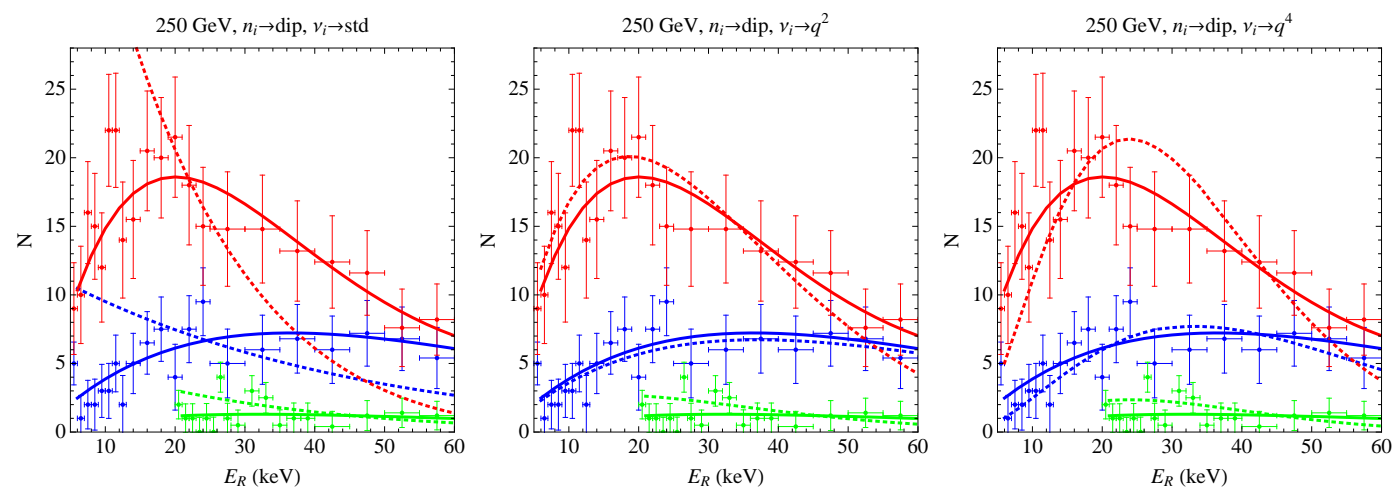

Figure 4.5: Fits of $\nu_{i}=\operatorname{std}, q^{2}$, and $q^{4}$ operators to $n_{i}=$ dipole data. The data are shown as points with Poisson error bars. The dipole best fit spectrum is shown as a solid line and the other operator best fit spectra are shown as dotted lines. Xenon is red, germanium is blue, and argon is green.

the anapole operator has a non-standard velocity-dependence, its spectrum is very similar to momentum independent scattering. Since the velocity dependence does not affect the spectrum and the $q^{2}$ piece is subdominant, the anapole and standard operators may be said to have the same momentum dependence. Likewise, the dipole and $q^{2}$ operators share their own momentum dependence.

Based on the collected plots in the appendix, we can extend these conclusions. For example, on argon, which has a high energy threshold and small event number, several incorrect operators can give a decent global fit to the data and a low value of $\widetilde{L}_{\text {min }} /$ d.o.f. Even on germanium the wrong operator can mimic the true operator if the momentum dependence of the operator differs by a power of $q^{2}$ or less. This is not true for xenon, which has a good $\widetilde{L}_{\min } /$ d.o.f. only for the correct operator. Thus the $\widetilde{L}_{\min } /$ d.o.f. test works for xenon but not for argon. Additionally, it appears evident from the figures collected in the appendix that the overlap test, combining data from all targets, also works very well for high mass candidates. Nonetheless, the overlap test described here is in some sense another manifestation of the $\widetilde{L}_{\text {min }}$ test. This comes about because of the relatively large number of events on the xenon target, which increase its value of $\widetilde{L}_{\text {min }}$ (since, for large event numbers, $\widetilde{L}_{\text {min }}$ scales 
with the number of events) and shrink its CLC relative to the other CLCs. The small xenon CLCs are in turn more precise in selecting a preferred region in parameter space, and thus are the single most important factor in the overlap test. In this way, the overlap and $\widetilde{L}_{\text {min }}$ tests are linked. This can even be seen in the $10 \mathrm{GeV}$ case, for example, by comparing the CLCs in the appendix from the $n_{i}=q^{2}$ and standard data sets. In the former case the xenon cross section is enhanced relative to germanium by the ratio of the xenon and germanium atomic ratios $A_{\mathrm{Xe}} / A_{\mathrm{Ge}}$, which increases the xenon sensitivity, shrinks its CLCs, and allows modest CLC separation. In the $n_{i}=$ standard case there is no such enhancement and no CLC separation is evident. Now we turn to investigating the impact of statistical effects on our conclusions by considering the case where all targets observe the same number of events.

\subsubsection{Operator Discrimination with Equal Event Numbers on All Targets}



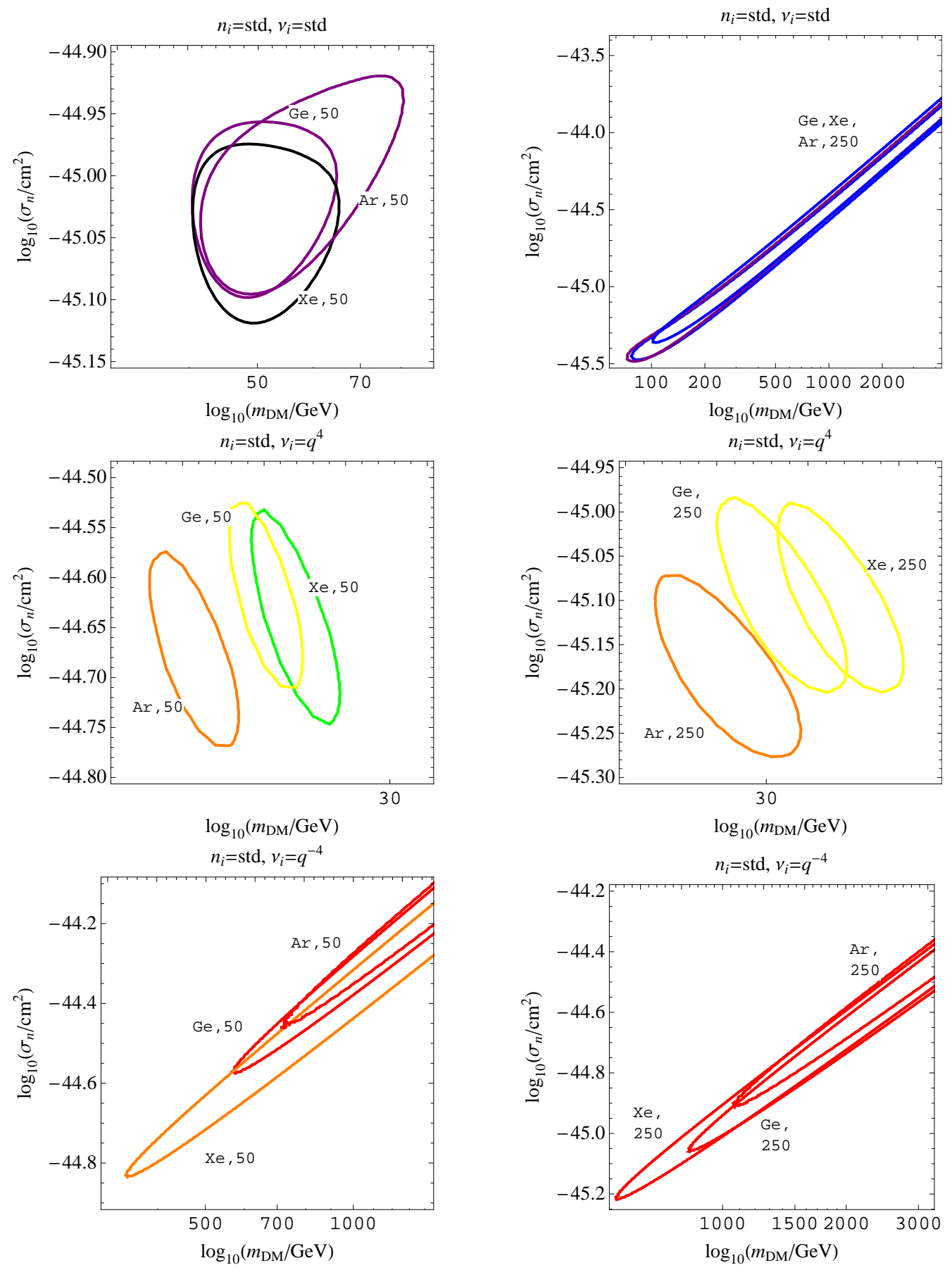

$\begin{array}{lll}0 & 0.5\end{array}$

1

1.5

2

4

Figure 4.6: 95\% CLCs for 10, 50, and $250 \mathrm{GeV}$ particles interacting through an $n_{i}=$ standard operator with 300 events on each target. Comparisons are made to $\nu_{i}=$ standard, $q^{4}$, and $q^{-4}$ operators. Colors as in Fig. 4.1. 

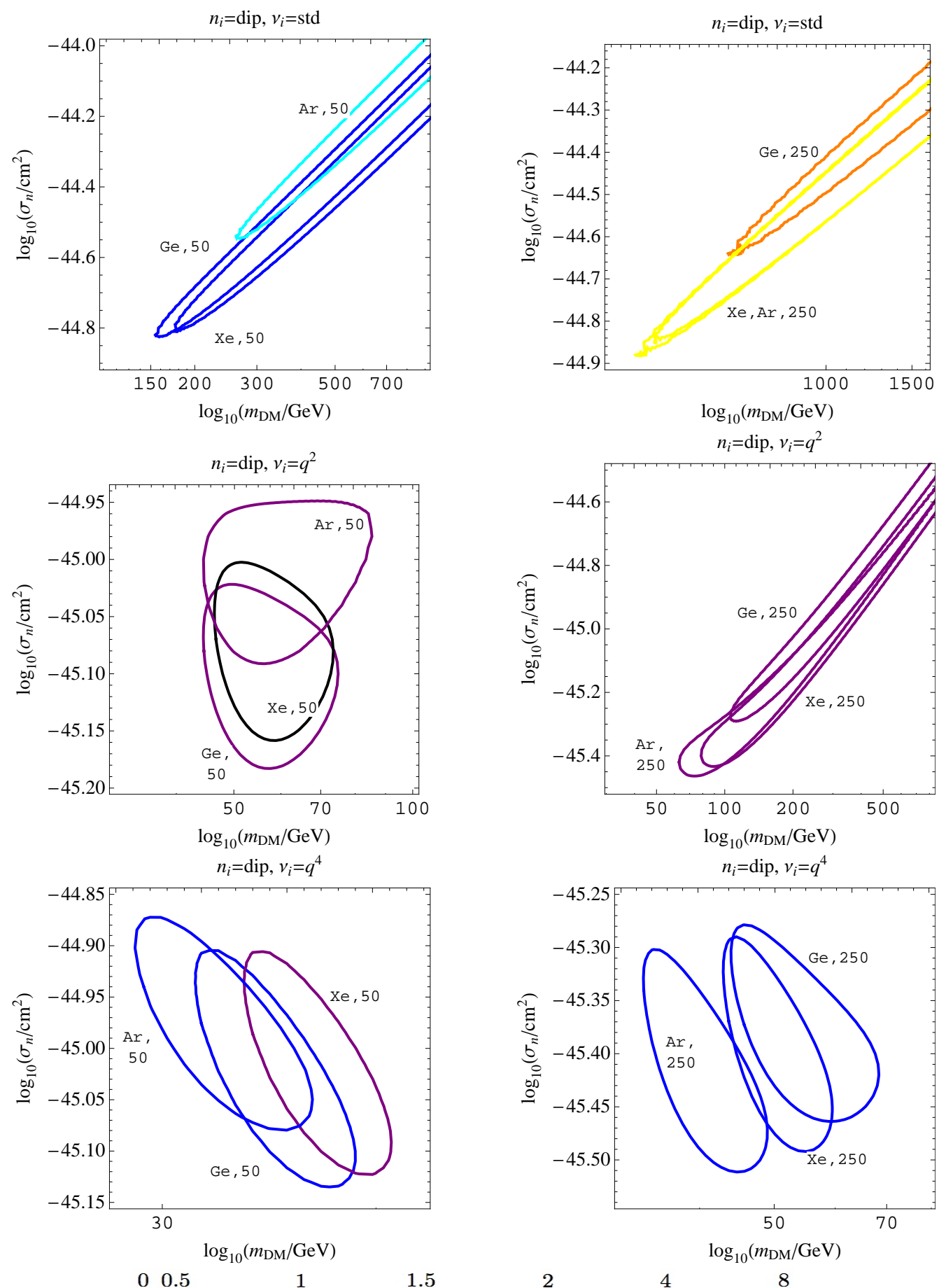

Figure 4.7: 95\% CLCs for a 10, 50, and $250 \mathrm{GeV}$ particles interacting through an $n_{i}=$ dipole moment operator with 300 events on each target. Comparisons are made to $\nu_{i}=$ standard, $q^{2}$, and $q^{4}$ operators. The colors represent the value of $\widetilde{L}_{\text {min }} /$ d.o.f. Compared to the case shown in Fig. (4.2) where exposures were fixed for all targets so that xenon was able to power the statistics, the ability to discriminate the dipole interaction from standard and $q^{4}$ operators using overlap or the values of $\widetilde{L}_{\min } /$ d.o.f. is diminished. 
In Figs. (4.6) and (4.7) we show the CLC plots for $50 \mathrm{GeV}$ and $250 \mathrm{GeV}$ candidates with 300 events on all targets to see if any discriminatory ability is lost. We omit the $10 \mathrm{GeV}$ case because of the poor discrimination; we have checked that even by increasing the number of events in the $10 \mathrm{GeV}$ candidate case by an order of magnitude, little improvement occurs. To achieve these event numbers we simply reduce the exposure for xenon; for argon we increase the exposure in addition to lowering the energy threshold from $20 \mathrm{keV}$ to $5 \mathrm{keV}$. This "equal event number" normalization is less physically motivated than the "equal exposure" normalization adopted above, but is necessary for understanding the robustness of our results.

Compared to the figures from the equal exposure scenario it is apparent that for high mass candidates the simple overlap test loses much of its capability to distinguish operators. There is no overlap when $n_{i}=$ standard and $\nu_{i}=q^{4}$, but there is overlap for $\nu_{i}=q^{-4}$. This problem is even more noticeable in the case with $n_{i}=$ dipole where with equal events there is mutual overlap in almost every instance. This loss of power is expected since the precision of the xenon CLCs has greatly diminished.

However, the $\widetilde{L}_{\min } /$ d.o.f. test remains generally strong and even increases in relevance for some combinations of operators. Previously, only xenon and germanium were capable of differentiating operators on the basis of the $\widetilde{L}_{\min } /$ d.o.f. test. By increasing the number of events on an argon detector we have a third viable test of the $\widetilde{L}_{\min } /$ d.o.f. of each operator. This compensates for the weakening of the overlap test that is a consequence of decreasing the number of events on xenon. Yet, even though we are still able to extract the correct operator, it seems that our standards for discrimination have been changed by the statistics.

We would like to be able to use the same test on any combination of operators, elements, and exposures. The fact that the overlap test is powered by the high $\widetilde{L}_{\text {min }}$ values of xenon is an important clue. We will show that what seems to be a qualitative difference in how we draw conclusions in the equal exposure and equal event scenarios 
is just an illusion.

\subsubsection{Comparison of Models}

In this subsection, we present significance tests for different trial operators. We combine all three data sets and define $\widetilde{L}_{\text {min }}^{\text {tot }}$ to be the minimum log-likelihood value from this global fit.
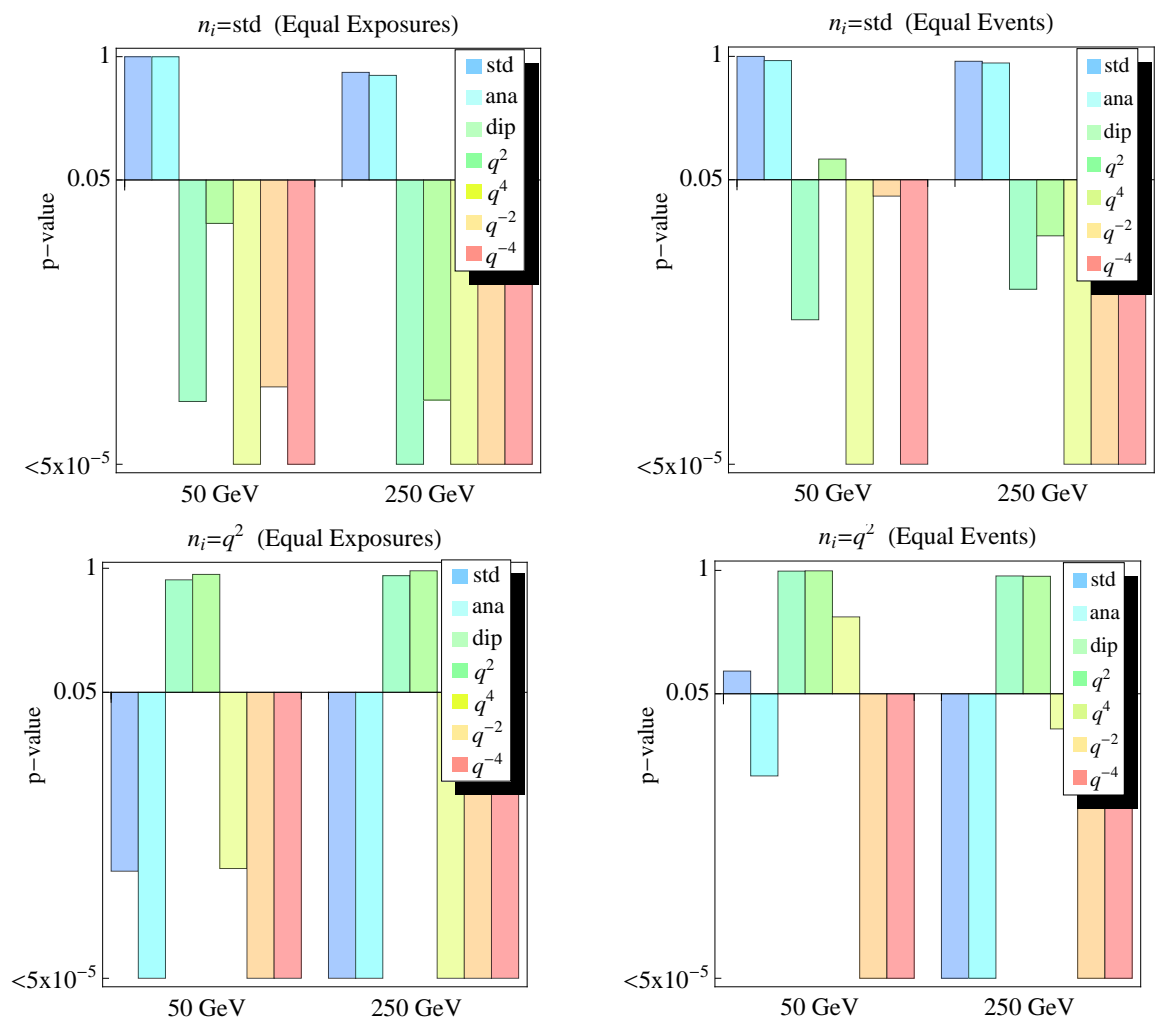

Figure 4.8: The p-values of trial operators for $n_{i}=$ standard and $q^{2}$ operators for candidate masses of 50 and $250 \mathrm{GeV}$. We display the equal exposure and equal event bar charts side by side to underscore the robustness of the discrimination. For the visual purpose, the plot is normalized so that each bar starts at 5\% significance level.

Here we make the approximation that $\widetilde{L}_{\text {min }}^{\text {tot }}$ values follow a $\chi^{2}$ distribution. This is valid when there are a large number of events, as in the cases studied here. Thus, 

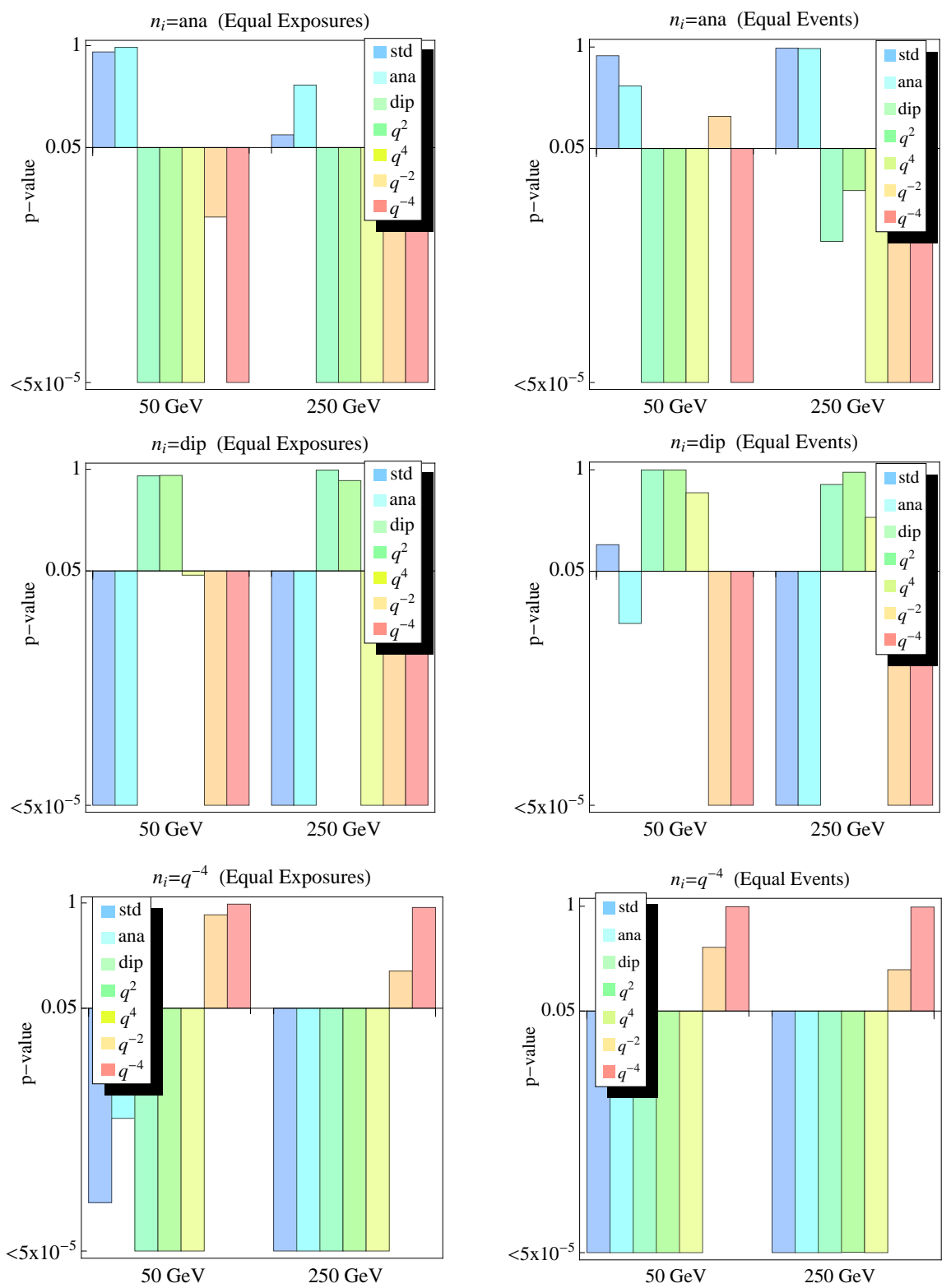

Figure 4.9: The p-values of trial operators for $n_{i}=$ anapole, dipole, and $q^{-4}$ operators for candidate masses of 50 and $250 \mathrm{GeV}$. We display the equal exposure and equal event bar charts side by side to underscore the robustness of the discrimination. For the visual purpose, the plot is normalized so that each bar starts at $5 \%$ significance level.

we may derive a $p$-value for any given trial operator by defining [38]

$$
p\left(\widetilde{L}_{\min }^{\mathrm{tot}}, n_{\mathrm{d}}\right)=\int_{\widetilde{L}_{\text {min }}^{\mathrm{tot}}}^{\infty} \frac{x^{n_{\mathrm{d}} / 2-1} e^{-x / 2}}{2^{n_{\mathrm{d}} / 2} \Gamma\left(n_{\mathrm{d}} / 2\right)} d x
$$


where $n_{\mathrm{d}}$ is the number of degrees of freedom; this is the total number of nonzero bins for all three experiments minus the number of fit parameters. For a given trial operator, the $p$-value describes the probability of producing a fit that has a $\widetilde{L}^{\text {tot }}$ larger than $\widetilde{L}_{\min }^{\text {tot }}$, so that a high $p$-value for an operator indicates that the relevant model is able to fit the data well.

We display results for the standard, anapole, dipole, $q^{2}$, and $q^{-4}$ data sets in Figs. (4.8) and (4.9). We omit the $10 \mathrm{GeV}$ data from this comparison because the discrimination is obviously quite poor, and we are primarily interested in seeing if the higher mass candidates are sensitive to the statistical effect of changing the event numbers seen by the argon and xenon targets. One immediately notices that the scattering of 50 and $250 \mathrm{GeV}$ candidates typically provides very good differentiation between correct and incorrect operators. In most of cases, we can reject the trial model at much better than 5\% significance level if operators do not match. Also as expected, we see that the standard and anapole operators are hard to distinguish, as are the $q^{2}$ and dipole operators. This is a consequence of the fact that the velocitydependent contribution to the composite operators' spectra has weak momentum dependence, and thus the anapole and standard operators have approximately momentum independent spectra just as the dipole and $q^{2}$ operators have the same $q^{2}$ momentum dependence.

Modulo this degeneracy, we see that it is possible to extract the particle physics nature of the dark matter scattering events given that future ton-scale direct detection experiments observe $\mathcal{O}(100)$ dark matter events. Moreover, the $p$-value is capable of extracting the momentum dependence of the operator equally well for the two event normalizations (equal exposures or equal event numbers on all targets) studied here. Finally, we also have checked that the results obtained from the $p$-value test derived from the global-fit $\widetilde{L}_{\min }^{\text {tot }}$ agree with the conclusions we would draw from calculating the $p$-values for each element individually, but the result from the global-fit has a 
better discrimination ability.

\subsection{Conclusions}

We have discussed the capability of direct detection experiments to extract the particle physics underlying DM scattering events. We found that Poisson noise limits the ability of a single detector to determine the momentum dependence of the operator mediating the scattering. When the exposures are equal for different elements and the data are from observations of high mass candidates, only xenon, due to its high atomic number, is capable of determining the momentum dependence of the interaction on its own. Under these conditions, examining the preferred regions from data generated by more than one element allows one to extract the correct momentum dependence; this can be seen in Figs. (4.1) and (4.2). When two operators give a similarly good fit to the data, it is because they have a very similar momentum dependence. We found that much of the power of this "overlap test" is derived from xenon's capability to discriminate operators. When all event numbers are held constant, so that the xenon exposure is decreased and the argon exposure is scaled up, we found that the overlap test is less powerful, but the combination of data from all three targets still provides good operator discrimination. We found that the minimum log-likelihood value for the global fit summarizes all of this information succinctly and robustly. The minimum log-likelihood value for the global fit is sensitive both to overlap and to the individual goodness of fit information, and can be put in one-to-one correspondence with the $p$-value, allowing us to make quantitative statements about the ability of each operator to fit the data. For a light DM candidate on the other hand, the effects

of the energy threshold, the energy resolution, and the scattering kinematics combine in such a way that the operator mediating the scattering cannot be extracted even for very high event numbers.

We thank Tim Cohen for discussions. The work of SDM and KMZ was sup- 
ported by NSF CAREER award PHY 1049896, and the work of HBY and KMZ was supported by NASA Astrophysics Theory Program grant NNX11AI17G. 


\section{Bibliography}

[1] G. Jungman, M. Kamionkowski, K. Griest, Phys. Rept. 267, 195-373 (1996). [hep-ph/9506380].

[2] E. Aprile et al. [XENON100 Collaboration], Phys. Rev. Lett. 105, 131302 (2010) [arXiv:1005.0380 [astro-ph.CO]].

[3] Z. Ahmed et al. [The CDMS-II Collaboration], Science 327, 1619-1621 (2010). [arXiv:0912.3592 [astro-ph.CO]].

[4] R. Bernabei, P. Belli, F. Cappella, R. Cerulli, C. J. Dai, A. d'Angelo, H. L. He, A. Incicchitti et al., Eur. Phys. J. C67, 39-49 (2010). [arXiv:1002.1028 [astroph.GA]].

[5] C. E. Aalseth et al. [CoGeNT collaboration], Phys. Rev. Lett. 106, 131301 (2011) [arXiv:1002.4703 [astro-ph.CO]]. C. E. Aalseth et al., "Search for an Annual Modulation in a P-Type Point Contact Germanium Dark Matter Detector," arXiv:1106.0650 [astro-ph.CO].

[6] G. Angloher, M. Bauer, I. Bavykina, A. Bento, C. Bucci, C. Ciemniak, G. Deuter, F. von Feilitzsch et al., [arXiv:1109.0702 [astro-ph.CO]].

[7] P. Sorensen, J. Angle, E. Aprile, F. Arneodo, L. Baudis, A. Bernstein, A. Bolozdynya, P. Brusov et al., [arXiv:1011.6439 [astro-ph.IM]].

[8] J. Angle et al. [ XENON10 Collaboration ], Phys. Rev. D80, 115005 (2009). [arXiv:0910.3698 [astro-ph.CO]].

[9] Z. Ahmed et al. [CDMS-II Collaboration], Phys. Rev. Lett. 106, 131302 (2011) [arXiv:1011.2482 [astro-ph.CO]].

[10] M. Pospelov, T. ter Veldhuis, Phys. Lett. B480, 181-186 (2000). [hep$\mathrm{ph} / 0003010]$. 
[11] A. Kurylov, M. Kamionkowski, Phys. Rev. D69, 063503 (2004). [hepph/0307185]. K. Sigurdson, M. Doran, A. Kurylov, R. R. Caldwell, M. Kamionkowski, Phys. Rev. D70, 083501 (2004). [astro-ph/0406355].

[12] J. Bagnasco, M. Dine, S. D. Thomas, Phys. Lett. B320, 99-104 (1994). [hep$\mathrm{ph} / 9310290]$.

[13] S. Chang, A. Pierce and N. Weiner, JCAP 1001 (2010) 006 [arXiv:0908.3192 [hep-ph]].

[14] H. An, S. -L. Chen, R. N. Mohapatra, S. Nussinov, Y. Zhang, Phys. Rev. D82, 023533 (2010). [arXiv:1004.3296 [hep-ph]].

[15] S. Chang, N. Weiner, I. Yavin, Phys. Rev. D82, 125011 (2010). [arXiv:1007.4200 [hep-ph]].

[16] A. L. Fitzpatrick, K. M. Zurek, Phys. Rev. D82, 075004 (2010). [arXiv:1007.5325 [hep-ph]].

[17] T. Banks, J. -F. Fortin, S. Thomas, [arXiv:1007.5515 [hep-ph]].

[18] S. D. McDermott, H. -B. Yu, K. M. Zurek, Phys. Rev. D83, 063509 (2011). [arXiv:1011.2907 [hep-ph]].

[19] E. Aprile et al. (XENON1T Collaboration), XENON1T at LNGS. Proposal, February (2010) and Technical Design Report, October (2010).

[20] M. Kos [DEAP/CLEAN Collaboration], "Dark Matter Detection with the Deap/Clean Detectors," PoS ICHEP2010 (2010) 455. http://deapclean.org/

[21] P. Brink, Marina del Rey (2010). http://www . physics .ucla.edu/hep/dm10/talks/brink.pd

[22] M. Drees, C. -L. Shan, JCAP 0806, 012 (2008). [arXiv:0803.4477 [hep-ph]]. C. -L. Shan, New J. Phys. 11, 105013 (2009). [arXiv:0903.4320 [hep-ph]]. 
L. E. Strigari, R. Trotta, JCAP 0911, 019 (2009). [arXiv:0906.5361 [astroph.HE]]. A. H. G. Peter, Phys. Rev. D81, 087301 (2010). [arXiv:0910.4765 [astroph.CO]]. P. J. Fox, G. D. Kribs and T. M. P. Tait, Phys. Rev. D 83, 034007 (2011) [arXiv:1011.1910 [hep-ph]]. P. J. Fox, J. Liu and N. Weiner, Phys. Rev. D 83, 103514 (2011) [arXiv:1011.1915 [hep-ph]]. M. Cannoni, J. D. Vergados, M. E. Gomez, Phys. Rev. D83, 075010 (2011). [arXiv:1011.6108 [hep-ph]]. C. L. Shan, [arXiv:1103.0481 [hep-ph]]. M. Pato, [arXiv:1106.0743 [astro-ph.CO]]. C. Strege, R. Trotta, G. Bertone, A. H. G. Peter and P. Scott, arXiv:1201.3631 [hep-ph].

[23] M. Pato, L. Baudis, G. Bertone, R. R. de Austri, L. E. Strigari, R. Trotta, [arXiv:1012.3458 [astro-ph.CO]].

[24] S. Eidelman et al., Physics Letters B592, 1 (2004).

[25] G. Fricke, C. Bernhardt, K. Heilig, L. A. Schaller, L. Schellenberg, E. B. Shera and C. W. de Jager, Atom. Data Nucl. Data Tabl. 60, 177 (1995).

[26] V. A. Bednyakov, F. Simkovic, Phys. Part. Nucl. 37, S106-S128 (2006). [hep$\mathrm{ph} / 0608097]$.

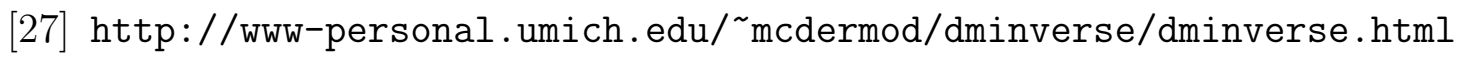




\section{CHAPTER V}

\section{Neutrino Phenomenology in a $3+1+1$ Framework}

Evidence continues to grow in the MiniBooNE (MB) antineutrino mode supporting a low-energy excess compatible with the MB neutrino mode and possibly also confirming the results of the LSND experiment. At least one sterile neutrino is required to explain the anomalies consistent with the observations of other experiments. At the same time, there is a strong tension between the positive signals of LSND and MB and the null results of $\nu_{e}$ and $\nu_{\mu}$ disappearance experiments. We explore a scenario, first proposed in [1], where the presence of an additional heavy sterile neutrino (with mass well above an $\mathrm{eV}$ ) can alleviate tension between LSND, MB and the null results of disappearance experiments. We compare and contrast this $3+1+1$ scenario with the more standard $3+1$ scenario and carry out global fits to all oscillation data including new $2011 \mathrm{MB} \bar{\nu}$ data. We find that the tension can be somewhat alleviated and that a phenomenologically viable window for the heavy neutrino, consistent with rare decays and $\mathrm{BBN}$ constraints, can be found if the fifth neutrino has a

Written in collaboration with Eric Kuflik and K. M. Zurek. Originally published as Neutrino Phenomenology in a 3+1+1 Framework, Phys. Rev. D 86, 033015 (2012). arXiv:1205.1791 [hep-ph], MCTP-12-11. 
mass of order $0.3-10 \mathrm{GeV}$. We also find, however, that the $2011 \mathrm{MB} \bar{\nu}$ data exacerbates the tension with null experiments in both the $3+1$ and $3+1+1$ models when the lowest energy bins are included, resulting in little improvement in the global fit. We also discuss the implications of an additional neutrino for the reactor and gallium anomalies, and show that an oscillation explanation of the anomalies is disfavored by cosmological considerations, direct searches, and precision electroweak tests.

\section{$5.1 \quad$ Introduction}

Neutrino masses imply the presence of new states that can generate neutrino mass terms consistent with the standard model (SM) $S U(2)$ gauge symmetry. Since the observed neutrino mass splittings are tiny, the standard way to implement the new states is to decouple them by giving them large masses. At energies relevant for neutrino experiments, this gives rise to a new higher-dimension operator which generates neutrino masses, presumably at the scale of grand unification. Since the dynamics of the new physics is decoupled, however, this mechanism for neutrino mass generation can never be directly tested.

Recent experimental hints have, on the other hand, suggested that there may be new dynamics in the neutrino sector at a much lower scale, leading to the possibility of probing the neutrino mass generation mechanism directly. The LSND [2] and MiniBooNE (MB) [3-5] experiments both have reported results consistent with oscillations through a new sterile neutrino mass eigenstate with a splitting that is larger than the splittings that control the oscillations of the SM neutrinos. The SM mass splittings are fixed by the observations in atmospheric and solar neutrino experiments to be $\mathcal{O}\left(10^{-3} \mathrm{eV}^{2}\right)$ and $\mathcal{O}\left(10^{-5} \mathrm{eV}^{2}\right)$, respectively. By contrast, the results from the LSND and MB $\bar{\nu}_{\mu} \rightarrow \bar{\nu}_{e}$ and $\nu_{\mu} \rightarrow \nu_{e}$ searches are consistent with a mass-squared splitting roughly between 0.1 and $1 \mathrm{eV}^{2}$, which would require a new, heavier neutrino 
mass eigenstate.

The simplest extension of the SM that can satisfy these requirements is a single sterile neutrino (the $3+1$ scheme). The existence of such a neutrino in the LSND and MB preferred mass region is, however, disfavored by global fits to the data [6], since null searches for neutrino disappearance tightly constrain the mixing angles needed to produce the LSND and MB signals. Measurements of $\bar{\nu}_{e}$ fluxes from nuclear reactors and $\bar{\nu}_{\mu}$ fluxes from beam dump experiments can be combined to reject the relatively large mixing angles required by LSND and MB. As we show below, this statement remains true even using the new reactor flux predictions as inputs. Thus, a new neutrino capable of explaining the combined neutrino oscillation data enters a very constrained parameter space.

In addition to these considerations, there are more complications facing the $3+1$ hypothesis. Early results from MB $[3,5]$ suggested that such a $3+1$ scheme might not have been compatible with the MB data alone, since the parameters needed to fit $\bar{\nu}_{\mu} \rightarrow \bar{\nu}_{e}$ and $\nu_{\mu} \rightarrow \nu_{e}$ appeared to be different: at high energy the MB anti-neutrino mode favored oscillations and was in better agreement with the $\bar{\nu}_{\mu} \rightarrow \bar{\nu}_{e}$ LSND data, while $\nu_{\mu} \rightarrow \nu_{e}$ data was consistent with a null result. ${ }^{1}$ The addition of a second sterile neutrino (the $3+2$ scheme) allows for $\mathrm{CP}$ violation which can reconcile differences in $\bar{\nu}_{\mu} \rightarrow \bar{\nu}_{e}$ and $\nu_{\mu} \rightarrow \nu_{e}$. However, this scheme suffers from a similar tension between the null data and the positive signals and does little to ameliorate the difficulties of the $3+1$ scheme $[6,7]$. In addition, new $\bar{\nu}$ data [8] from MB, shown in Fig. 5.1, indicates that the apparent difference between the $\nu$ and $\bar{\nu}$ modes is disappearing, thereby obviating one of the primary appeals of the $3+2$ framework.

Still, the tension between the null results and the LSND and MB data persists. In this paper we consider a simple scheme, proposed in [1], designed to alleviate the tension between the LSND and MB positive signals and the null results from

\footnotetext{
${ }^{1} \mathrm{~A}$ low-energy excess in the $\nu_{\mu}$ channel was initially suspected of being a systematic effect [3] and was reported to be incompatible with a neutrino oscillation interpretation [4].
} 

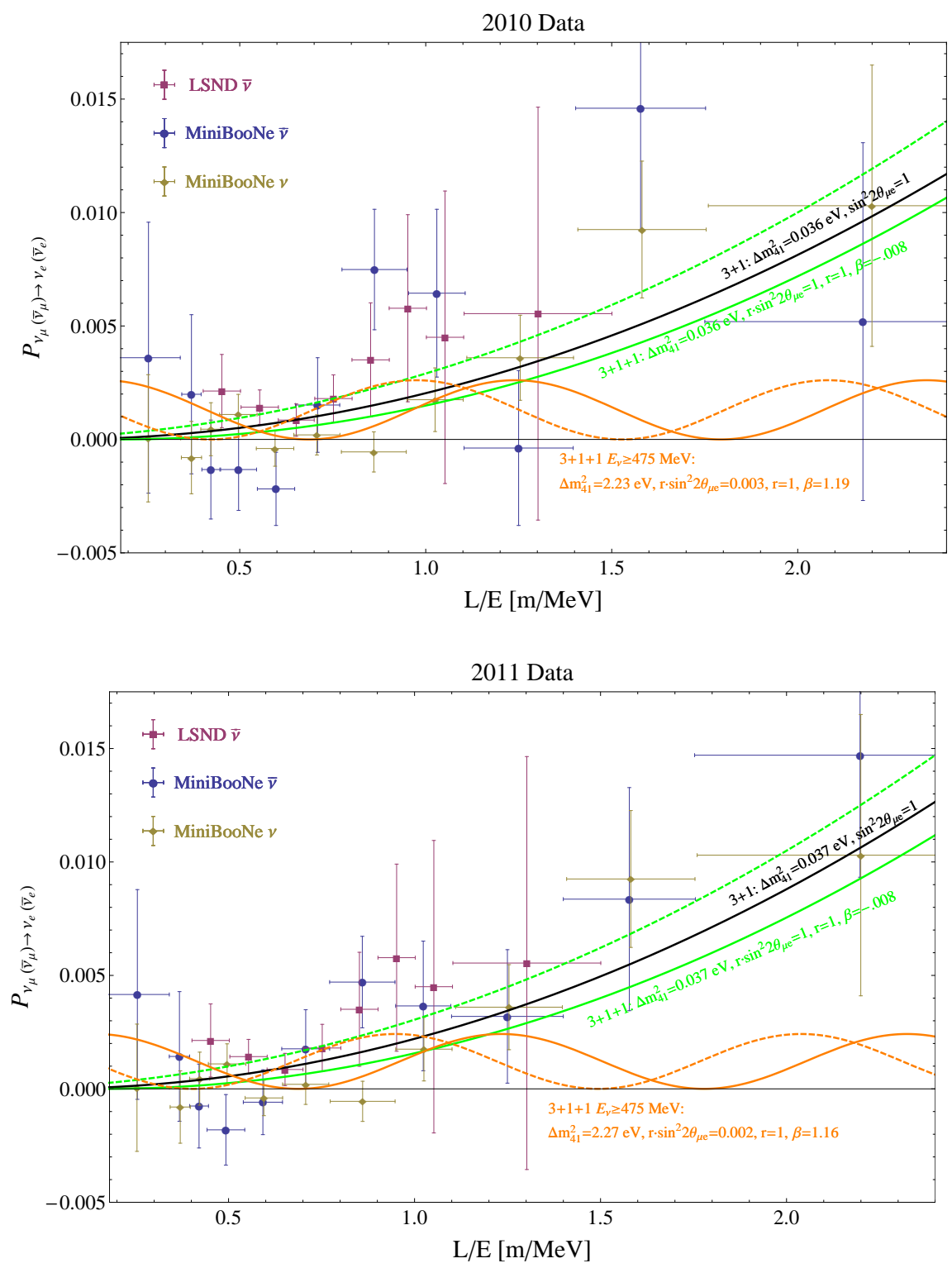

Figure 5.1: Comparison of 2010 (upper panel) and 2011 (lower panel) MB $\bar{\nu} L / E$ data with MB $\nu$ and LSND $\bar{\nu} L / E$ data. In both panels, the MB $\nu$ data is taken from [4]. In the upper panel, the MB $\bar{\nu}$ data is taken from [5], while in the lower panel the MB $\bar{\nu}$ data has been updated with the results of [8]. We show the best fit lines in the $3+1$ scenario (black), the $3+1+1$ scenario (green) using all data points, and the $3+1+1$ scenario (orange) dropping the three low-energy data points so that the data is in the range $E_{\nu}>475 \mathrm{MeV}$. In all plots, $\nu$ lines are dotted and $\bar{\nu}$ lines are solid.

reactor and short baseline experiments. This scenario requires a single light sterile neutrino with mass splitting in the MB and LSND range between 0.1 and $1 \mathrm{eV}^{2}$ and 
a second much heavier $\left(\Delta m^{2} \gg 1000 \mathrm{eV}^{2}\right)$ neutrino whose oscillations are averaged over. The heavier neutrino participates directly or indirectly in both disappearance and appearance experiments. Because most disappearance experiments have their first detector relatively far from the neutrino production point the heavy neutrino has undergone many oscillations before reaching the detector, and the effect of the heavy neutrino is to change the flux of the initial flavor neutrinos. If this flux is not precisely known, as is true in many reactor experiments, the experiment is relatively insensitive to oscillations through the heavy neutrino. Appearance experiments, by contrast, look for the appearance of a new flavor in a pure initial flavor beam, so they are sensitive to oscillations through the heavy neutrinos. In this way, if the initial neutrino flux is not very well known in the disappearance experiments, new parameter space may open for the appearance experiments, giving rise to the possibility that the positive signals from LSND and MB are no longer in conflict with the results from otherwise null experiments.

The purpose of this paper is two-fold. First, we examine the $3+1$ scenario in light of the new MB $\bar{\nu}$ data. This improves the compatibility of the combined appearance data within the $3+1$ framework, but we find that the best fit region shifts considerably to larger mixings and smaller mass splittings, which increases the tension with the null experiments. Second, we explore the phenomenology of, and present constraints on, the " $3+1+1 "$ framework of $[1,9]$. We will examine exactly how and to what extent the fifth neutrino is able to have an effect on the allowed parameter space of the fourth neutrino.

The outline of this paper is as follows. We begin by establishing our notation and conventions. We then carry out fits for the $3+1$ scenario in light of new data from MB, complete with constraints from a diverse set of null experiments. We then turn to discussing the parameter space for the $3+1+1$ scenario with respect to neutrino experiments, before analyzing in detail the constraints from BBN, astrophysics and 
rare decays, which constrain the fifth neutrino to have a mass $\sim 0.3-10 \mathrm{GeV}$. In section IV we present aspects of some models that explicitly realize the features of the $3+1+1$ scenario, and we conclude in section $\mathrm{V}$.

\subsection{Phenomenology Of Sterile Neutrino Models}

We establish our notation and contrast the $3+1$ framework (see e.g. $[6,7,10,11]$ ) with the $3+1+1$ scheme $[1,9]$. We will lay out some conventions for discussing these models, leaving a more complete discussion of statistical methods and derivation of the oscillation formulae in the $3+1+1$ scenario to the appendix.

We aim to examine the oscillation appearance and disappearance data in depth, with a specific emphasis on the new conclusions to be drawn from some recently presented preliminary MB data [8]. This new data is in better agreement with the LSND data and prefers a sterile neutrino with lower mass and more substantial mixing than indicated by the earlier MB data.

\subsubsection{Conventions}

We parameterize the mass mixing by

$$
\nu_{\alpha}=\sum_{i=1}^{N} U_{\alpha i} n_{i}
$$

where $\nu_{\alpha}$ are the neutrino flavor eigenstates, which include the 3 left-handed (active) neutrinos of the SM plus any $S U(2)$-singlet (sterile) neutrinos; $U_{\alpha i}$ are the elements of a unitary $N \times N$ matrix that diagonalizes the neutrino mass matrix and causes mixing between the neutrino flavor eigenstates; and $n_{i}$ are the neutrino mass eigenstates with mass $m_{i}$ ordered by increasing mass.

Since we are focusing on a $3+1$ scheme (with a single light neutrino) and a $3+1+1$ scheme (with one light and one heavy neutrino) we are generally interested in oscil- 
lation probabilities where all but one of the mass eigenstates are easily kinematically accessible. From Eq. (C.3), the probability of detecting $\nu_{\beta}$ in a $\nu_{\alpha}$ beam is

$$
\begin{aligned}
P_{\nu_{\alpha} \rightarrow \nu_{\beta}}= & \delta_{\alpha \beta}\left[1+2(a-1)\left|U_{\alpha 5}\right|\left|U_{\beta 5}\right|\right]+(1-a)\left|U_{\alpha 5}\right|^{2}\left|U_{\beta 5}\right|^{2} \\
& -4 \sum_{5>i>j} \Re\left\{U_{\alpha i}^{*} U_{\beta i} U_{\alpha j} U_{\beta j}^{*}\right\} \sin ^{2} x_{i j}-4 a \sum_{j=1}^{4} \Re\left\{U_{\alpha 5}^{*} U_{\beta 5} U_{\alpha j} U_{\beta j}^{*}\right\} \sin ^{2} x_{5 j} \\
& -2 \sum_{5>i>j} \Im\left\{U_{\alpha i}^{*} U_{\beta i} U_{\alpha j} U_{\beta j}^{*}\right\} \sin 2 x_{i j}-2 a \sum_{j=1}^{4} \Im\left\{U_{\alpha 5}^{*} U_{\beta 5} U_{\alpha j} U_{\beta j}^{*}\right\} \sin 2 x_{5 j} .
\end{aligned}
$$

Here $x_{i j}=\Delta m_{i j}^{2} L / 4 E=1.27 \frac{\left(m_{i}^{2}-m_{j}^{2}\right) L / E}{\mathrm{eV}^{2} \mathrm{~m} / \mathrm{MeV}}$, where $L$ is the distance the neutrino has traveled and $E$ is the neutrino energy. Since $n_{5}$ will be much heavier than the other neutrinos, accounting for the possibly suppressed production of and oscillation through $n_{5}$ requires a phase space factor $a$ that interpolates from 0 (kinematically forbidden) to 1 (phase space fully accessible) as a function of the neutrino energy. For the short baselines and high energies of the experiments under consideration it will be a good approximation to take $x_{i j} \simeq 0$ for $i$ and $j=1,2,3$, and this formula simplifies considerably. For instance, the probability for disappearance of flavor $\alpha$ is

$$
1-P_{\nu_{\alpha} \rightarrow \nu_{\alpha}}=\sin ^{2} 2 \theta_{\alpha 4} \sin ^{2} x_{41}+2\left|U_{\alpha 5}\right|^{2}\left(1-\frac{a+1}{2}\left|U_{\alpha 5}\right|^{2}\right)
$$

where we define $\sin ^{2} 2 \theta_{\alpha 4}=4\left|U_{\alpha 4}\right|^{2}\left(1-\left|U_{\alpha 4}\right|^{2}-\left|U_{\alpha 5}\right|^{2}\right)$ and we assume that the characteristic oscillation length associated with $\Delta m_{51}^{2}$ is so short that $\sin ^{2} x_{51} \rightarrow \frac{1}{2}$ holds over the volume of the detector. Experiments that probe disappearance of $\nu_{e}$ are carried out at reactors and in solar neutrino searches, while $\nu_{\mu}$ disappearance is probed by beam dump and atmospheric neutrino experiments.

Following [1], the probability for $\nu_{e}$ appearance in a $\nu_{\mu}$ beam, measured by LSND and MB among others, simplifies. From Eq. (C.4)

$$
P_{\nu_{\mu}\left(\bar{\nu}_{\mu}\right) \rightarrow \nu_{e}\left(\bar{\nu}_{e}\right)}=\sin ^{2} 2 \theta_{\mu e} \sin ^{2}\left(x_{41} \pm \beta\right)+\kappa,
$$


with the definitions

$$
\begin{aligned}
\sin ^{2} 2 \theta_{\mu e} & =4\left|U_{\mu 4}\right|^{2}\left|U_{e 4}\right|^{2} r \\
\kappa & =\left|U_{\mu 4}\right|^{2}\left|U_{e 4}\right|^{2}\left\{(1-r)^{2}+a\left[(1-r)^{2}+4 r \sin ^{2} \beta\right]\right\}
\end{aligned}
$$

where $+(-)$ is for $\nu(\bar{\nu})$ oscillations,

$$
\begin{aligned}
r & \equiv\left|U_{\mu 4}^{*} U_{e 4}+U_{\mu 5}^{*} U_{e 5}\right| /\left|U_{\mu 4}^{*} U_{e 4}\right| \\
\beta & \equiv \frac{1}{2} \tan ^{-1}\left(\frac{\sin \phi\left|U_{e 5}\right|\left|U_{\mu 5}\right|}{\left|U_{e 4}\right|\left|U_{\mu 4}\right|+\cos \phi\left|U_{e 5}\right|\left|U_{\mu 5}\right|}\right)
\end{aligned}
$$

and $\phi \equiv \arg \left(\frac{U_{e 5} U_{\mu 5}^{*}}{U_{e 4} U_{\mu 4}^{*}}\right) . \beta$ is the CP-odd parameter that can account for differences in $\nu$ and $\bar{\nu}$ oscillations. The $3+1$ model can be recovered in the limit $U_{e 5}$ and $U_{\mu 5} \rightarrow 0$, or $r=1$ and $\kappa=\beta=0$. We emphasize that the sensitivity to the mixings with $n_{5}$ is such that even the limit $a \rightarrow 0$ produces nontrivial oscillation effects.

The $3+1+1$ model is capable of opening parameter space closed by $3+1$ models because of the possibility of CP violation and because in general we can have $r>1$. In the small mixing, CP-conserving limit the effect of $r$ is multiplicative because we may make the approximation $\sin ^{2} 2 \theta_{\mu e} \simeq r \sin ^{2} 2 \theta_{e 4} \sin ^{2} 2 \theta_{\mu 4} / 4$, and the limits from disappearance experiments can be made compatible with larger appearance mixings if one has $r>1$. However, we will show that because of the presence of the term that depends on $\left|U_{e 5}\right|^{2}$ in Eq. (5.3) the constraints on the mixings with $n_{5}$ are almost as strong as the constraints on the mixings with $n_{4}$. This forces $r$ to be close to 1 for most of the interesting parameter space, and $r$ is not effective in practice for reconciling the appearance and disappearance experiments.

\subsubsection{Fits to Neutrino Appearance Anomalies}

Fits to the $3+1$ and $3+1+1$ frameworks with all relevant data are shown in Fig. 5.2; we display them side by side to enhance comparisons of the fits. In each panel we superimpose the results using the 2010 [5] and 2011 [8] $\bar{\nu}$ data from MB. We use 
the 2009 data [4] for the $\nu$ mode for all fits. The best fit to the data, using either the 2010 or $2011 \mathrm{MB} \bar{\nu}$ data, indicates a new sterile neutrino described by a mass splitting $\Delta m_{41}^{2} \sim \mathcal{O}\left(0.03 \mathrm{eV}^{2}\right)$ and a mixing angle roughly of size 1 , although the $\chi^{2}$ is relatively shallow and is consistent with mass splitting $\Delta m_{41}^{2} \sim \mathcal{O}\left(0.5 \mathrm{eV}^{2}\right)$ and mixing angle $\sim \mathcal{O}\left(3 \times 10^{-3}\right)$. These values differ from those we would find if we omitted the low-energy $\mathrm{MB} \nu$ and $\bar{\nu}$ points. As we discuss in more detail below, dropping these points reduces the significance of the signal so that the data are compatible with no oscillations at the $99 \%$ level, as noted in [8]. Because much of the significance of the fit to oscillations is derived from events with $E_{\nu}<475 \mathrm{MeV}$, we do not omit these points in our fits.

In principle, both appearance and disappearance oscillation experiments can bound the LSND and MB preferred region. We consider null appearance searches at KARMEN [12], E776 [13], NOMAD [14], CCFR [15], and NuTeV [16], and we find that the preferred region using the new MB $\bar{\nu}$ data is no longer in tension with these searches, due to the lower-mass preferred region. Although the LSND and MB oscillation results are not strongly constrained by the null appearance searches, the mixing angle probed by the appearance experiments can be tightly constrained by combining the results of $\nu_{e}$ and $\nu_{\mu}$ disappearance experiments. The disappearance experiments independently constrain $\sin ^{2} 2 \theta_{e 4}$ and $\sin ^{2} 2 \theta_{\mu 4}$, and in a $3+1$ scenario with small mixing angles we can approximate $\sin ^{2} 2 \theta_{\mu e} \simeq \sin ^{2} 2 \theta_{e 4} \sin ^{2} 2 \theta_{\mu 4} / 4$, so we obtain limits on the LSND and MB parameter space by combining the two sets of constraints. Details of how these constraints are combined are given in the appendix. The $\nu_{e}$ disappearance constraints include short-baseline reactor experiments with new reactor flux predictions $[17]^{2}$ as well as constraints from the ratio of flux observed in the Bugey $40 \mathrm{~m}$ and $15 \mathrm{~m}$ detectors [18]. Disappearance of $\nu_{\mu}$ is constrained by CDHS [19] and CCFR [20]

\footnotetext{
${ }^{2}$ The new reactor flux has been reported to reflect oscillations of a sterile neutrino, but we find that it is not consistent with our preferred region, and we use the reactor data as a constraint. We discuss a possible resolution to this anomaly below.
} 
at high mass. We also take into account mass-independent unitarity constraints arising from the maximal measurement of the atmospheric $\left(\nu_{\mu}\right)$ [21] disappearance mixing angles made by the Super-Kamiokande experiment. In the appendix we show that this leads to $\left|U_{\mu 4}\right|^{2}+\left|U_{\mu 5}\right|^{2}<0.0175(0.0274)$ at $90 \%(99 \%)$ confidence.
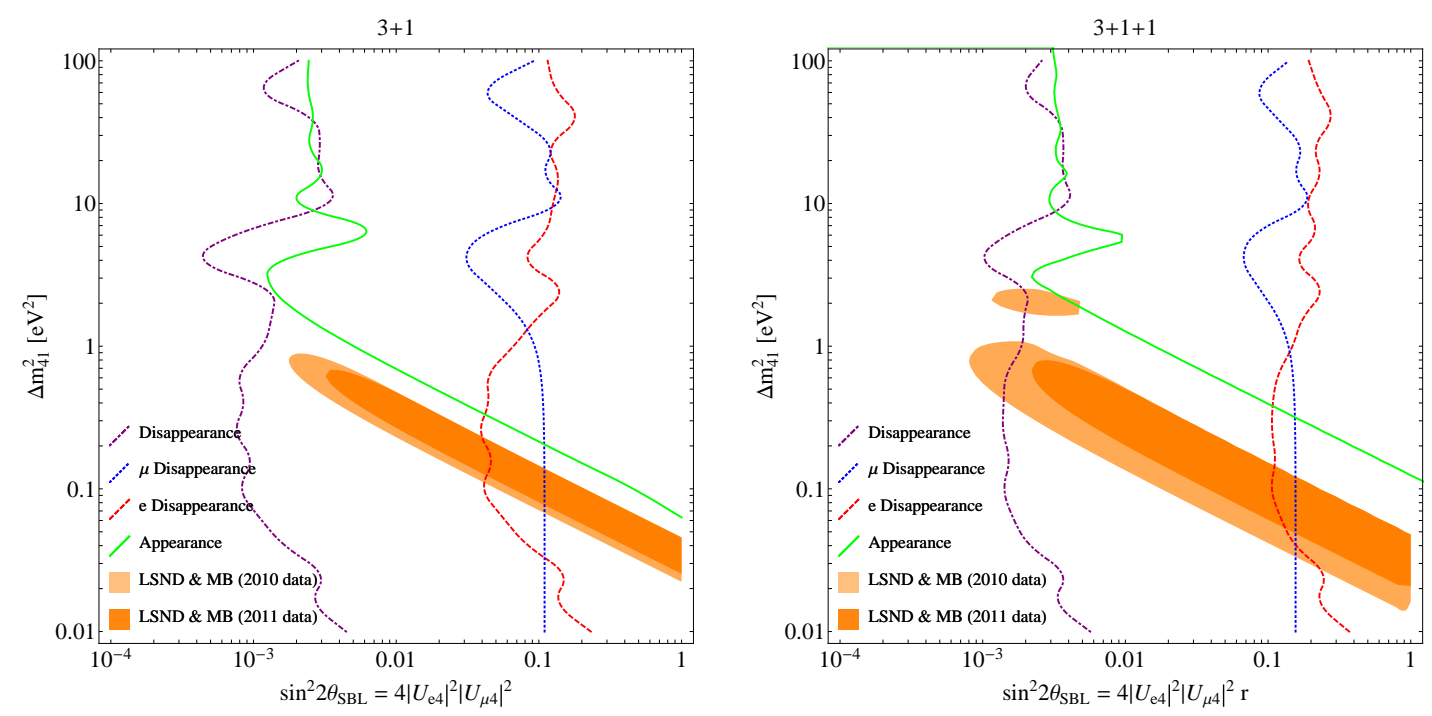

Figure 5.2: Fits in the $3+1$ (left) and $3+1+1$ (right) neutrino models. We also contrast the allowed regions using the 2010 (light-orange) and the 2011 (darkorange) MB $\bar{\nu}$ data. In both panels we show the appearance allowed region at $99 \%$ as well as the appearance null result and disappearance null result exclusion curves at $99 \%$. There is significant tension with the disappearance experiments and oscillations reported by LSND and MB for both the $3+1$ and $3+1+1$ scenarios with the 2011 data.

The best fit oscillation statistics for the $3+1$ scheme are given in Table 5.1 , where we show the $\chi_{\min }^{2}$ values for the disappearance and appearance data sets individually as well as the $\chi_{\min }^{2}$ for the global data set. The value of the $\chi_{\min }^{2} /$ DOF for the global fit does not indicate a bad fit to the data (as noted in, e.g., [10]) but the $\chi_{\mathrm{PG}}^{2}[22]$ value for the different data sets is very high, which indicates that the data as a whole are not compatible. This is reflected in Fig. 5.2, which shows the "disappearance" curve ruling out the "LSND \& MB" allowed region. The new data play an important role in shifting the preferred region and increasing the tension between appearance and disappearance: we find that the appearance data on its own is marginally more 


\begin{tabular}{|c|c|c|c|c|c|}
\hline \multicolumn{3}{|c|}{2010 Data } & \multicolumn{3}{|c|}{2011 Data } \\
\hline & $\chi_{\min }^{2}$ & bins & & $\chi_{\min }^{2}$ & bins \\
\hline Disappearance & 25.4 & 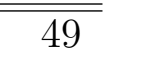 & Disappearance & 25.4 & 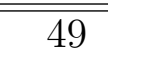 \\
\hline Appearance & 0.20 & 5 & Appearance & 0.20 & 5 \\
\hline $\mathrm{LSND}+\mathrm{MB}$ & 32.1 & 30 & $\mathrm{LSND}+\mathrm{MB}$ & 24.0 & 30 \\
\hline Everything & 75.4 & 84 & Everything & 72.9 & 84 \\
\hline $\begin{array}{l}\mathrm{G}=\left(\sum \chi^{2}\right)_{\min } \\
\mathrm{p} \text {-value }=5.02 \times\end{array}$ & $\sum \chi_{\mathrm{m}}^{2}$ & $\begin{array}{l}=17.7 \\
18 \sigma)\end{array}$ & $\begin{array}{l}\chi_{\mathrm{PG}}^{2}=\left(\sum \chi^{2}\right)_{\min } \\
\quad \mathrm{p} \text {-value }=3.44>\end{array}$ & $2 x$ & $\begin{array}{l}=23.3 \\
14 \sigma)\end{array}$ \\
\hline
\end{tabular}

Table 5.1: Fits to the $3+1$ framework using 2010 and $2011 \bar{\nu}$ data. With the new MB data, the appearance and disappearance experiments disagree at more than the $4 \sigma$ level.

self-consistent when incorporating the $2011 \mathrm{MB} \bar{\nu}$ data $\left(\chi_{\min , \mathrm{LSND}+\mathrm{MB} 2011}^{2}=24.0\right)$ instead of the 2010 data $\left(\chi_{\min , \mathrm{LSND}+\mathrm{MB} 2010}^{2}=32.1\right)$, while the parameter goodness of fit becomes slightly worse $\left(\chi_{\mathrm{PG}, 2010}^{2}=17.7\right.$ and $\left.\chi_{\mathrm{PG}, 2011}^{2}=23.3\right)$. This is a result of a more significant departure from the null oscillation hypothesis at large $L / E$ in the 2011 data, which is compatible with the excess at low energy found in the MB $\nu$ data, as can be seen in Fig. 5.1. Due to the increased power at large $L / E$, our global appearance region is at somewhat lower mass and higher mixing than shown by previous global fits (e.g., [6]). We conclude that the tension between the positive signals and the null searches indicates that a single sterile neutrino is very unlikely to explain the entirety of the collected data.

In the right panel of Fig. 5.2 we show the results of a similar analysis performed in the $3+1+1$ framework, where the fit region differs from the the $3+1$ case because of the CP-odd phase $\beta$ and the multiplicative factor $r$. The parameter $r$ represents a potentially significant handle in the $3+1+1$ framework, since it can give a multiplicative enhancement of the appearance angle compared to the disappearance angles. For small $\beta, r$ effectively measures the magnitude of the mixings with $n_{5}$, and to obtain the desired enhancement over the $3+1$ scheme we need the mixings with $n_{5}$, and thus the value of $r$, to be greater than 1. However, as shown in the left panel of Fig. 5.3, 

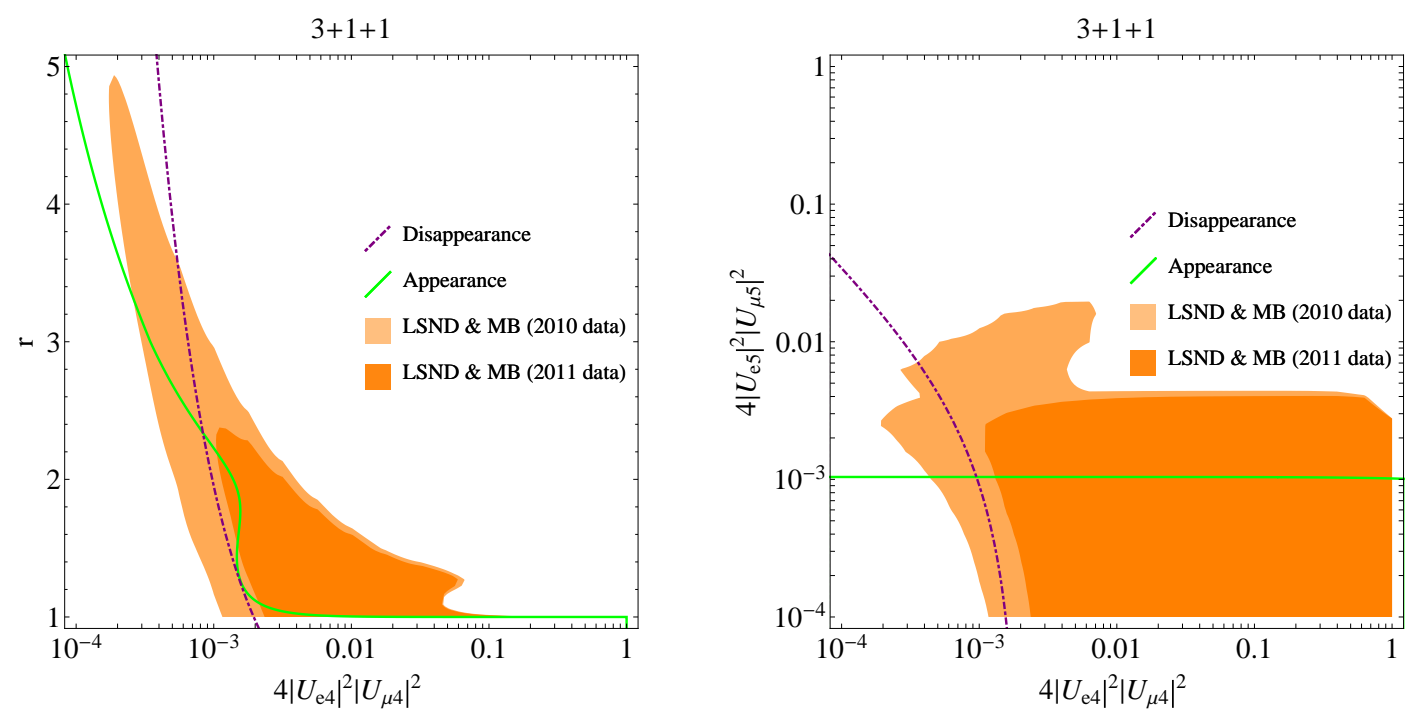

Figure 5.3: Constraints on $r$ as a function of $\left|U_{e 4}\right|^{2}\left|U_{\mu 4}\right|^{2}$ for $0.04 \mathrm{eV}^{2}<\Delta m_{41}^{2}<6 \mathrm{eV}^{2}$. We see that $r$ is close to 1 in the appearance preferred region, and has limited ability to reduce the tension with null experiments.

we find that $r$ is bounded by the null experiments to be very close to 1 for most of the values of $\left|U_{e 4}\right|^{2}$ and $\left|U_{\mu 4}\right|^{2}$ favored by LSND and MB. This is a consequence of the "zero-distance" effect [23], which allows for the oscillation of neutrino flavors at arbitrarily low distances. The zero-distance effect can manifest itself in two ways in the experiments in consideration. First, disappearance experiments bound the sum of the mixing angles due to this effect, as in Eq. (5.3), which forces either $\left|U_{\alpha 5}\right|^{2}$ or $\left|U_{\alpha 4}\right|^{2}$ to be small. Thus, disappearance experiments constrain $r$ to be very close to 1 for large $\left|U_{\alpha 4}\right|^{2}$, as is true in the appearance preferred region. This effect is shown in the left panel of Fig. 5.3, where $r$ is seen to be essentially compatible with 1 in the entire appearance preferred region. The second way the zero-distance effect would be visible is as a positive offset in the appearance probability. However, the appearance experiments exhibit transition probabilities roughly of order $0.5 \%$, which allows them to place their own firm upper bound on $\left|U_{e 5}\right|^{2}\left|U_{\mu 5}\right|^{2}$, as is visible in the right panel of Fig. 5.3. In other words, we find that $r$ is negligibly effective in reconciling the 

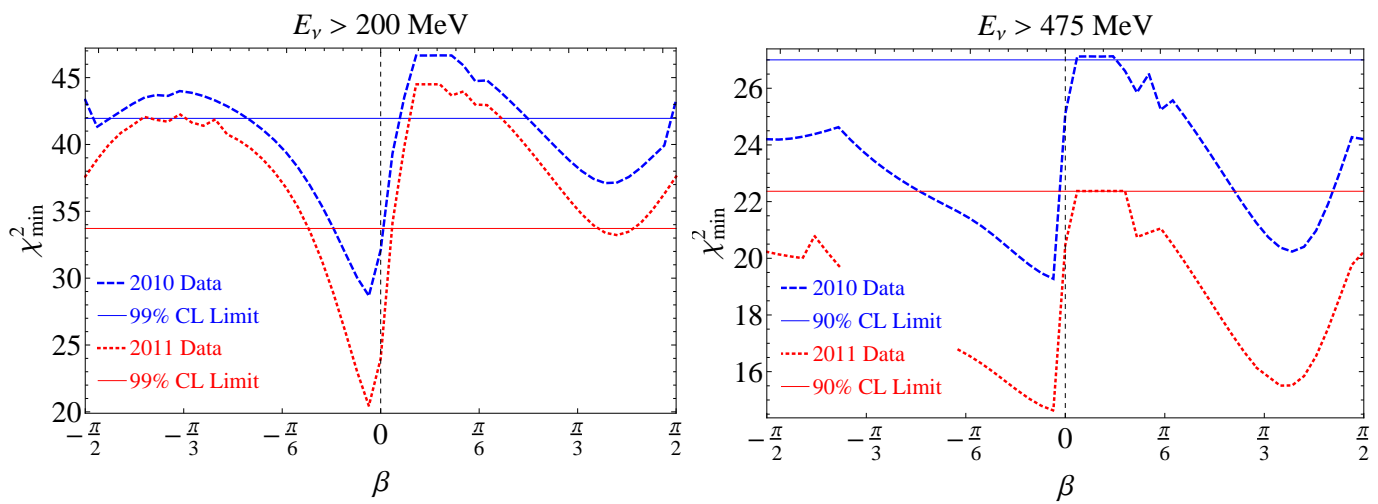

Figure 5.4: $\chi^{2}$ as a function of the CP-odd parameter $\beta$, with (left) and without (right) the $\mathrm{MB} \nu$ and $\bar{\nu}$ data for $200 \mathrm{MeV}<E_{\nu}<475 \mathrm{MeV}$. We show fits utilizing both the 2010 and 2011 MB $\bar{\nu}$ data, and we show the $90 \%$ or $99 \%$ allowed value from the $\Delta \chi^{2}$ test that we use.

appearance and disappearance data sets.

The other potential advantage of the $3+1+1$ framework is the possibility of CP violation, but we also find that this is not very effective in reducing the tension with the null experiments. As shown in the left panel of Fig. 5.4, the $\chi^{2}$ has a pronounced preference for a small nonzero value of $\beta$, and the sharpness of this feature means that the extra parameter freedom is largely unimportant in defining our preferred region. When we drop the $\mathrm{MB} \nu$ and $\bar{\nu}$ data points below $475 \mathrm{MeV}$, as advocated in the initial $\mathrm{MB}$ data release [3], we find that $\mathrm{CP}$ violation has a much more significant impact on the fit. This is because the $\chi^{2}$ is substantially flatter as a function of $\beta$ and exhibits two rather broad and nearly degenerate minima, as shown in the right panel of Fig. 5.4. This in turn is a result of the mostly flat spectrum of the $\nu$ and $\bar{\nu}$ data points in the region $E_{\nu}>475 \mathrm{MeV}$, which can be made compatible at the $1 \sigma$ level for a wide range of low-mixing-angle oscillations when $\mathrm{CP}$ violation is allowed. The ultimate effect of the shallower $\chi^{2}$ is to reduce the preference for a particular 
mass or mixing, which opens up a wider range of parameter space and broadens the preferred region. When we perform fits analogous to those in Fig. 5.2 for the data with $E_{\nu}>475 \mathrm{MeV}$, we find that the significance of the signal drops so drastically that the remaining data are consistent with no oscillations at the $99 \%$ level, as noted in [8]. We show the CP-violating best fits to the $E_{\nu}>475 \mathrm{MeV}$ data alongside the best fits to the $E_{\nu}>200 \mathrm{MeV}$ data in Fig. 5.1.

\begin{tabular}{|c|c|c|c|c|c|}
\hline \multicolumn{3}{|c|}{2010 Data } & \multicolumn{3}{|c|}{2011 Data } \\
\hline & $\chi_{\min }^{2}$ & bins & & $\chi_{\min }^{2}$ & bins \\
\hline Disappearance & 24.6 & $\overline{49}$ & Disappearance & 24.6 & 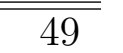 \\
\hline Appearance & 0.20 & 5 & Appearance & 0.20 & 5 \\
\hline $\mathrm{LSND}+\mathrm{MB}$ & 28.3 & 30 & $\mathrm{LSND}+\mathrm{MB}$ & 19.4 & 30 \\
\hline Everything & 74.4 & 84 & Everything & 73.2 & 84 \\
\hline $\begin{array}{l}{ }_{\mathrm{G}}=\left(\sum \chi^{2}\right)_{\min } \\
\mathrm{p} \text {-value }=1.6 \times\end{array}$ & $\chi_{\mathrm{m}}^{2}$ & $\begin{array}{l}=21.3 \\
6 \sigma)\end{array}$ & $\begin{array}{l}\chi_{\mathrm{PG}}^{2}=\left(\sum \chi^{2}\right)_{\min } \\
\quad \text { p-value }=6.0 \times\end{array}$ & $\sum_{-5} \chi$ & $\begin{array}{l}=29.0 \\
00 \sigma)\end{array}$ \\
\hline
\end{tabular}

Table 5.2: Results of fits to the $3+1+1$ framework using 2010 and $2011 \mathrm{MB} \bar{\nu}$ data. With the new data, the appearance and disappearance data sets still disagree at about the $4 \sigma$ level, with only slight improvement over the $3+1$ case.

Finally, we give the best fit statistics for the $3+1+1$ model in Table 5.2, taking $E_{\nu}>200 \mathrm{MeV}$ as usual. As in the $3+1$ case, the $2011 \mathrm{MB} \bar{\nu}$ data provides slightly more agreement in the combined appearance data than the 2010 data. Again, the global fit to the data gives an acceptable $\chi^{2} / \mathrm{DOF}$, but the PG test underscores the point that the data sets are incompatible. The p-value for the $\chi_{\mathrm{PG}}^{2}$ in the $3+1+1$ case is slightly lower than in the $3+1$ model for both the 2010 and $2011 \mathrm{MB} \bar{\nu}$ data. This suggests some improvement in agreement, but with the new data the tension remains at the $4 \sigma$ level in both the $3+1$ and $3+1+1$ cases. 


\subsection{Phenomenology of the Heavy Neutrino $n_{5}$ in the $3+1+1$ Scheme}

We discuss the parameter space of interest for the heavy neutrino, $n_{5}$, that appears in the $3+1+1$ framework. If $n_{5}$ is a Majorana neutrino the neutrinoless double beta decay constraints are extremely restrictive [24], so we will take $n_{5}$ to be a Dirac state.

We begin by showing how some experimental anomalies recently reported at low significance could plausibly be explained by the existence of this heavy mass eigenstate. Then we proceed to place constraints on its parameter space. Tension with $\mathrm{BBN}$ constraints forces us to the regime where $m_{5} \gtrsim 1 \mathrm{MeV}$. Above this region constraints from SN1987A, pion and kaon decays, beam dump experiments, and nonobservation of $\mu \rightarrow e \gamma$ enter. We find that if one wishes to use $n_{5}$ to explain experimental anomalies, only a small window with $m_{5} \sim \mathcal{O}(1 \mathrm{GeV})$ and mixing $\left|U_{e 5}\right|\left|U_{\mu 5}\right| \sim \mathcal{O}\left(10^{-2}\right)$ is allowed, with the additional requirement that $n_{5}$ be stable on collider timescales or decay to non-SM final states.

\subsubsection{The Gallium and Reactor Anomalies}

In recent years, several experiments have reported observing anomalously low neutrino fluxes. Since these anomalies include many different energy and distance scales and exhibit no $L / E$ dependence, we do not include them in the fits to $n_{4}$, whose mass splitting will cause visible oscillations at these experiments. Instead, we fit to this data with the heavy neutrino $n_{5}$ whose oscillations are averaged over in all experiments. A somewhat more detailed discussion of the anomalies we fit with $n_{5}$ oscillations is given in the appendix.

The most statistically significant of these position- and energy-independent anoma-

lies is the reactor antineutrino anomaly $[17,25]$ (RAA), where the global average of the observed $\bar{\nu}_{e}$ flux is less than anticipated by a factor $R_{\mathrm{RAA}}=0.943 \pm 0.023$. In 
addition, anomalously low measurements of the $\nu_{e}$ scattering gallium cross-section [26], the gallium anomaly (GA), may indicate disappearance of $\nu_{e}$. The deficit based on four measurements of the average $\nu_{e}$ scattering cross section from the process $\nu_{e}+{ }^{71} \mathrm{Ga} \rightarrow{ }^{71} \mathrm{Ge}+e^{-}$is, with correlated errors taken to be those in [17], $R_{\mathrm{GA}}=0.86 \pm 0.06$. Independent measurements of the strength of the relevant Gamow-Teller transitions [27] supports the conclusion that this deficit might be due to averaged oscillations of a heavy neutrino. Finally, measurements of the energy dependence of the scattering cross section in the process $\nu_{e}+{ }^{12} \mathrm{C} \rightarrow{ }^{12} \mathrm{~N}_{\text {g.s. }}+e^{-}[28]$ are very mildly discrepant with the cross-section predictions [29] and might also be due to a similar reduction of flux of $\nu_{e}$. This was originally presented as a constraint on the GA parameter space in [30], but the shallowness of the respective $\Delta \chi^{2}$ 's and the similarity in the parameter space leads us to consider the possibility of reconciling this data with the RAA and GA data.

Since the disappearance formulae are not sensitive to $\mathrm{CP}$ violation, the probability for both $\nu_{e}$ and $\bar{\nu}_{e}$ disappearance is given in Eq. (5.3). In all of the experiments in consideration, the neutrino energy is less than what we will find is the allowed range for $m_{5}$, so we set $a \rightarrow 0$ in all cases. It is clear from Eq. (5.3) that $n_{5}$ can reduce the $\nu_{e}$ flux by a fixed amount with no energy or distance dependence whether or not $n_{5}$ is kinematically accessible. To extract the most conservative limits on the mixings with $n_{5}$ we will set $\sin ^{2} 2 \theta_{e 4}$ to 0 in these fits. We fit to all of the available data using correlation information as in the literature. We find a preferred value of

$$
\left|U_{e 5}\right|^{2}=0.036 \pm 0.013 .
$$

Thus, we find that the RAA and GA may be consistently reconciled with the carbon data in the presence of a very heavy neutrino with averaged oscillations and a mixing angle of the magnitude indicated by Eq. (5.7). However, we will show below that for 
a very massive sterile neutrino, a mixing angle of this magnitude is disfavored by a combination cosmological considerations, direct searches, and precision electroweak tests.

\subsubsection{Big Bang Nucleosynthesis}

Depending on the mixing and mass, additional light sterile neutrino(s) (with mass $\lesssim 1 \mathrm{MeV}$ ) can be thermalized in the time leading up to big bang nucleosynthesis $(\mathrm{BBN})$. The presence of additional neutrinos at BBN can drive a faster expansion rate, modifying the abundance of the light elements, and in particular of helium. Thus, detailed observations of primordial elemental abundances from BBN can constrain the properties of sterile neutrinos. To set bounds, we require that the total number of neutrinos at $\mathrm{BBN}$ is less than $4.4[31]$, or $\Delta N_{\nu} \leq 1.4$. With potentially two sterile neutrinos with masses below $1 \mathrm{MeV}$ in the $3+1+1$ scenario, $\mathrm{BBN}$ constraints must be carefully checked. While constraints from BBN can be alleviated by the inclusion of a large lepton asymmetry (which effectively delays the time when an MSW-like coherent conversion can occur) [32], this mechanism becomes ineffective for the large mass splittings of interest for the $3+1+1$ model. We review the constraints in this section and apply them to the $3+1+1$ scenario.

We begin this discussion by reviewing the calculation for one active plus one sterile neutrino. With this result in hand, we will be able to easily see how the result extends to two sterile neutrinos (with widely separated masses) mixed with more than one active neutrino. We follow the density matrix formalism of [95]. Assuming that the active neutrinos are always in a fully thermalized state, the evolution equations for an arbitrary number of neutrinos $N$ is

$$
i \dot{\rho}=[\mathcal{H}, \rho]-i \Gamma \rho
$$


where the Hamiltonian $\mathcal{H}_{\alpha \beta}=V_{\alpha \beta}+\sum_{i=1}^{N} U_{\alpha i} U_{\beta i}^{*} m_{i}^{2} / 2 E$, and we take the production rate to be $\Gamma_{\alpha \beta}=\left(\Gamma_{\alpha}+\Gamma_{\beta}\right) / 2$, with $V_{i s}=\Gamma_{s}=0$ for all $i$. Specializing to the case of one active and one sterile neutrinos, the relevant equations are

$$
\begin{aligned}
& H x \partial_{x} \rho_{s s}=i \mathcal{H}_{a s}\left(\rho_{a s}-\rho_{s a}\right) \\
& H x \partial_{x} \rho_{a s}=-i\left[\left(\mathcal{H}_{a a}-\mathcal{H}_{s s}\right)-i \gamma_{a s}\right] \rho_{a s}+i \mathcal{H}_{a s}\left(\rho_{a a}-\rho_{s s}\right),
\end{aligned}
$$

with $x=m / T$, and $m$ fixed to be $1 \mathrm{MeV}$. The effect of interactions encapsulated in $\gamma_{a s}$ is to damp away the coherent off-diagonal element, $\rho_{a s}$. Thus if $\gamma_{a s}$ is large we are forced into the stationary point where $\partial_{x} \rho_{a s} \approx 0$ [95], so that

$$
\rho_{a s}=\frac{\mathcal{H}_{a s}}{\left(\mathcal{H}_{a a}-\mathcal{H}_{s s}\right)-i \gamma_{a s}}\left(\rho_{a a}-\rho_{s s}\right)
$$

Substituting this in the differential equation for $\rho_{s s}$ we obtain

$$
H x \partial_{x} \rho_{s s}=\frac{\gamma_{a}}{4}\left(\rho_{a a}-\rho_{s s}\right) \frac{\sin ^{2} 2 \theta}{\left(\cos 2 \theta-V_{a a} / \delta E\right)^{2}+\gamma_{a}^{2} / 4 \delta E^{2}},
$$

where $\delta E=\Delta m^{2} / 2 E, \gamma_{a}=g_{a} \frac{180 \zeta(3)}{7 \pi^{4}} G_{F}^{2} T^{4} p$ and $V_{a a} \simeq-C_{a} G_{F}^{2} T^{4} p / \alpha$, with $g_{\nu_{e}} \simeq 4$, $g_{\nu_{\mu}, \nu_{\tau}} \simeq 2.9, C_{\nu_{e}} \simeq 0.61, C_{\nu_{\mu}, \nu_{\tau}} \simeq 0.61$. If we neglect the $\gamma_{a}^{2}$ term in the denominator, which is valid for the non-resonance case, this is easily soluble analytically, since the result takes on the simple form:

$$
\ln \left(1-\Delta N_{\nu}\right) \approx \frac{\gamma_{a}(T=m)}{4 H(T=m)} \sin ^{2} 2 \theta \int_{0}^{1} \mathrm{~d} x \frac{x^{8}}{\left(x^{6} \cos 2 \theta-V_{a a}(T=m) / \delta E(T=m)\right)^{2}},
$$

Doing the integral analytically or numerically, we see that the result scales as $\sim$ $\sqrt{\delta E(T=m) / V_{a a}(T=m)} \sim \sqrt{\Delta m^{2}}$. The physical meaning of this result is clear. The rate with which the sterile neutrino is populated is suppressed at late time because the interaction rate is dropping as $1 / x^{5}$. At the same time, the sterile neutrino is most 
likely to be populated when the mass splitting between the active and sterile states is smallest. The medium dependent mass splitting, however, is also dropping with $V_{a a}$. Altogether, the integral is dominated by when $x^{6} \cos 2 \theta \sim\left|V_{a a}(T=m) / \delta E(T=m)\right|$, so that the total result (squared) is [95]

$$
\begin{aligned}
\left(\Delta m_{41}^{2} / \mathrm{eV}^{2}\right) \sin ^{4} 2 \theta_{e s} & =3.2 \times 10^{-5} \ln ^{2}\left(1-\Delta N_{\nu}\right) \\
\left(\Delta m_{41}^{2} / \mathrm{eV}^{2}\right) \sin ^{4} 2 \theta_{\mu, \tau s} & =1.7 \times 10^{-5} \ln ^{2}\left(1-\Delta N_{\nu}\right) .
\end{aligned}
$$

With these physical insights, it is easy to see how the results generalize to the cases with more than one sterile or active neutrino. First, we can see that because the sterile neutrino is populated around when $V_{a a} / \delta E \sim 1$, at any given temperature only one of the sterile neutrinos will be populated if the masses of the sterile neutrinos are widely separated in mass from each other and from the active neutrinos. Thus, if we make the assumption that $m_{a} \ll m_{4} \ll m_{5}$, we can decouple the fourth and fifth neutrinos from each other and treat them as being populated only through their interactions with the active neutrinos.

The other complication to consider is mixing between the active neutrinos themselves. However, if the active neutrino mass splittings themselves are negligible in comparison to the sterile neutrino mass splittings, $\Delta m_{12}^{2} \ll \Delta m_{23}^{2} \ll \Delta m_{34}^{2} \ll \Delta m_{45}^{2}$, then the SM mixing angles $\theta_{12}, \theta_{13}, \theta_{23}$ can be rotated away, and as a result the mixing between the active neutrinos itself decouples. Thus we conclude that the constraints on active-sterile mixing can be decoupled accordingly, and we have

$$
\begin{aligned}
\left(\Delta m_{(4,5) 1}^{2} / \mathrm{eV}^{2}\right) \sin ^{4} 2 \theta_{e 4,5} & =3.2 \times 10^{-5} \ln ^{2}\left(1-\Delta N_{\nu}\right) \\
\left(\Delta m_{(4,5) 1}^{2} / \mathrm{eV}^{2}\right) \sin ^{4} 2 \theta_{\mu, \tau 4,5} & =1.7 \times 10^{-5} \ln ^{2}\left(1-\Delta N_{\nu}\right)
\end{aligned}
$$

Now LSND and MB, in the standard $3+1$ scenario, probe $\sin ^{2} 2 \theta_{\mu e}=4\left|U_{e 4}\right|^{2}\left|U_{\mu 4}\right|^{2} \simeq$ $\frac{1}{4} \sin ^{2} 2 \theta_{e 4} \sin ^{2} 2 \theta_{\mu 4}$, which is a good approximation in the small mixing angle limit. 
Thus we are able to conclude that

$$
4\left(\Delta m_{(4,5) 1}^{2} / \mathrm{eV}^{2}\right) \sin ^{2} 2 \theta_{\mu e}=\sqrt{3.2 \times 1.7} \times 10^{-5} \ln ^{2}\left(1-\Delta N_{\nu}\right),
$$

so that we learn that the sterile neutrino is thermally populated if it has mixing angles large enough to explain LSND plus MB in the $3+1$ scenario.

Now these results are easily extended to the $3+1+1$ scenario. Then we have constraints on $r \sin ^{2} 2 \theta_{\mu e}=4\left|U_{e 4}^{*} U_{\mu 4}\right|\left|U_{e 4}^{*} U_{\mu 4}+U_{e 5}^{*} U_{\mu 5}\right|$ from LSND plus MB. To alleviate the constraints from the disappearance experiments, we require $\left|U_{e 5}^{*} U_{\mu 5}\right| \gtrsim$ $\left|U_{e 4}^{*} U_{\mu 4}\right|$. Since $m_{5} \gg m_{4}$, we conclude from Eq. (5.15) that if $\nu_{4}$ is populated, then $\nu_{5}$ is also populated at BBN temperatures, unless $m_{5} \gtrsim 1 \mathrm{MeV}$. Since $\Delta N_{\nu}<1.4$, we thus conclude that most of the $m_{5}$ parameter space proposed in [1] is not consistent with the constraints from BBN, eliminating the entire region of parameter space with $33 \mathrm{eV}<m_{5} \lesssim 1 \mathrm{MeV}$.

Above the upper end of this mass range production of heavy sterile neutrinos may be inefficient at $\mathrm{BBN}$ temperatures, and the additional neutrino $n_{5}$ may not represent a fully populated degree of freedom. Because the state $n_{4}$ is fully populated, we require the fractional population of the state $n_{5}$ to satisfy $\Delta N_{\nu}^{\left(n_{5}\right)} \lesssim 0.4$ as calculated from Eq. (5.15). After their production is frozen out, the remnant $n_{5}$ will decay through charge- and neutral-current interactions at rates suppressed by the mixing parameters. If these decays proceed through SM channels, the decays of $n_{5}$ can add entropy and ionizing energy to the thermal bath at the time of BBN, spoiling predictions of the relic helium abundance. These considerations allow us to rule out the range

$$
6.2 \times 10^{-10}\left(\frac{m_{5}}{\mathrm{MeV}}\right)^{-1} \leq\left|U_{e 5} U_{\mu 5}\right| \lesssim 10^{1.5}\left(\frac{m_{5}}{\mathrm{MeV}}\right)^{-3.5},
$$

where the upper bound is a rough fit to the numerical analysis conducted in [34] (we display the numerical values in Fig. 5.5). These bounds extend up to $m_{5} \simeq m_{\pi}$, at 
which point new decay channels open which have not been analyzed numerically.

In the next section we consider further constraints from colliders on such heavy neutrinos. We will find that to satisfy the constraints, $n_{5}$ must have exotic invisible decays which are not via its SM mixing with the active neutrinos.

\subsubsection{Supernova $1987 \mathrm{~A}$}

The duration of the observed neutrino burst from Supernova 1987A (SN1987A) constrains the mass and couplings of any massive sterile neutrino. If the sterile neutrinos mix strongly enough that they are produced but are coupled weakly enough that they are not tightly bound to the supernova core they will allow too much energy to escape from the core, reducing the observed duration of the blast.

There are both lower and upper bounds on the neutrino coupling [96]. The lower bound comes from requiring that $n_{5}$ are efficiently produced in the interior of the supernova. If these neutrinos are efficiently produced and have low enough mixing, they will free stream out of the supernova and conduct energy away from the core too quickly. With larger mixing angles, the neutrinos will have a short mean free path and, for large enough mixing, they will be trapped in the supernova. If they are trapped but their mean free path is larger than the supernova core they will cause anomalous cooling of the star: blackbody radiation will be emitted from a region larger than the supernova core, and the supernova will cool too quickly. Because production of neutrinos in supernovae are dominated by charge-current processes, we find that $\nu_{e}$ production dominates $\nu_{\mu}$ production [36]. This gives slightly weaker bounds on the mixing angle $U_{\mu 5}$, and, because maximal mixing angles are in principle allowed by these arguments, we find that the lower bound on $U_{\mu 5}$ (which is approximately 5 times weaker than the lower bound on $\left.U_{e 5}\right)$ is in fact the lower bound on the product $\left|U_{e 5} U_{\mu 5}\right|$. For $m_{5} \gtrsim 0.1 \mathrm{MeV}$, where the matter effect becomes unimportant, we find that mixing angles $3.0 \times 10^{-5} \lesssim\left|U_{e 5} U_{\mu 5}\right| \lesssim 5.0 \times 10^{-3}$ are ruled out by these energy 
considerations. These bounds apply to $n_{5}$ regardless of its couplings.

The trapping argument given above will not apply for large mixing angles if $n_{5}$ decays invisibly to products with no SM interactions, as naturally considered in the model building section below. This is because, for the widths calculated below, we find that the decay length $L \simeq 10^{-10} \mathrm{~m}\left(10 \mathrm{MeV} / m_{5}\right)$ is much shorter than the mean free path $\lambda_{\mathrm{mfp}} \simeq 0.1 \mathrm{~m} / \sin ^{2} 2 \theta_{m}$ for the masses and mixings of interest. Therefore $n_{5}$ will decay well before it is trapped, and the bounds can no longer be lifted at very large mixing angles. These exclusions are model-dependent because they rely on the unknown couplings of the decay products, but since it is possible that $n_{5}$ evades the upper bounds described at large mixing angles we shade this region gray in the left panel of Fig. 5.5.

These bounds are also lifted for lighter sterile neutrinos since the relevant pro-

duction mechanism is matter-enhanced flavor transitions. For $m \lesssim \mathcal{O}(0.1 \mathrm{MeV})$ the bounds weaken and go to zero around $m \sim \mathcal{O}(100 \mathrm{eV})$ [96], so SN1987A bounds do not constrain $n_{4}$.

\subsubsection{Bounds from Light Mesons}

There are a variety of searches for exotic meson decays that produce strong bounds on the mass and mixing of $n_{5}$. We group these into a few categories as follows and display the collected results in Fig. 5.5.

Measured $\pi$ meson branching fraction: The pion branching ratio to $\mu$ and $e$ is $R_{\pi}=|\overline{\mathcal{M}}|_{\pi \rightarrow e \nu}^{2} /|\overline{\mathcal{M}}|_{\pi \rightarrow \mu \nu}^{2}$. At tree level, the matrix element $|\overline{\mathcal{M}}|_{\pi \rightarrow \ell_{\alpha} \nu}^{2}$ goes like

$$
|\overline{\mathcal{M}}|_{\pi \rightarrow \ell_{\alpha} n}^{2} \propto \sum_{i=1}^{N_{a}}\left|U_{\alpha i}\right|^{2}\left(m_{\alpha}^{2}+m_{i}^{2}\right)\left[m_{\pi}^{2}-\left(m_{\alpha}+m_{i}\right)^{2}\right]
$$


where $m_{\alpha}$ is the mass of the charged lepton $\ell_{\alpha}$. In the SM, where $m_{i}=0$ for all neutrinos and $U_{\ell_{\alpha} i}=\delta_{\ell_{\alpha} i}, R_{\pi}$ simplifies considerably. The most current SM calculation of this quantity to two loops is $R_{\pi}^{\mathrm{SM}, \text { th. }}=(1.2352 \pm 0.0001) \times 10^{-4}[37]$, while the best experimental bounds give $R_{\pi}=(1.230 \pm 0.004) \times 10^{-4}[38]$.

In the $3+1+1$ framework $R_{\pi}$ will differ depending on the mass range, so the constraints are piecewise. They simplify at high mass, where $m_{5} \gtrsim m_{\pi}-m_{\mu} \gg m_{e}$, which is near where the SN1987A bounds stop. We find

$$
\frac{R_{\pi}}{R_{\pi}^{\mathrm{SM}, \text { th. }}} \simeq\left\{\begin{array}{cl}
\frac{1-\left|U_{e j}\right|^{2}}{1-\left|U_{\mu 5}\right|^{2}}+\frac{\left|U_{e 5}\right|^{2}}{1-\left|U_{\mu 5}\right|^{2}} \frac{m_{5}^{2}}{m_{e}^{2}} \frac{m_{\pi}^{2}-m_{5}^{2}}{m_{\pi}^{2}-m_{e}^{2}} & m_{\pi}-m_{\mu} \lesssim m_{5} \lesssim m_{\pi} \\
\frac{1-\mid U_{e} 5}{1-\left|U_{\mu 5}\right|^{2}} & m_{5} \gtrsim m_{\pi}
\end{array}\right.
$$

The measured ratio is $R_{\pi} / R_{\pi}^{\mathrm{SM}, \text { th. }}=0.996 \pm 0.003$, so at $99 \%$ confidence we require that $R_{\pi} / R_{\pi}^{\mathrm{SM}, \text { th. }} \lesssim 1.004$. Thus, the mixing angles $U_{e 5}$ and $U_{\mu 5}$ are bounded fairly strongly in the intermediate mass range. We do a scan over the full parameter space and for each value of $m_{5}$ we find the maximum product of the mixing angles consistent with this constraint.

Muon lifetime: For nonzero $U_{e 5}$ and $U_{\mu 5}$, the total charged current interactions with the muon and electron below the muon mass will be reduced. The muon lifetime $\tau_{\mu}$ will be increased relative to the SM prediction due to the non-unitarity in the neutrino mixing matrix. In practice, Fermi's constant, $G_{F}$, is measured most precisely from measurements of $\tau_{\mu}$ [39], so one can derive constraints by comparing to an independent measurement of $G_{F}$. Following [40], we relate $M_{Z}, M_{W}$ and $\alpha$ to $G_{F}$ by

$$
G_{F}^{\prime}=\frac{\pi \alpha M_{Z}^{2}}{\sqrt{2} M_{W}^{2}\left(M_{Z}^{2}-M_{W}^{2}\right)(1-\Delta r)} .
$$

where $\Delta r=0.0362 \pm 0.0005[38]$ is the correction to the tree-level relationship. The values of $M_{Z}$ and $M_{W}$ used should be taken from purely kinematic measurements 
since other fits to $M_{W}$ include the measurements of $G_{F}$ from muon decay. We take $M_{W}=80.387 \pm 0.016 \mathrm{GeV}[41]$ and $M_{Z}=91.1875 \pm 0.0021 \mathrm{GeV}$ [42]. Plugging in these values, we find $G_{F}^{\prime}=(1.1679 \pm 0.0013) \times 10^{-5} \mathrm{GeV}^{-2}$. Comparing this to the value extracted from measurements of $\tau_{\mu}, G_{F}=1.166353(9) \times 10^{-5} \mathrm{GeV}^{-2}$ [39], we find for $m_{5}>m_{\mu}$

$$
\frac{G_{F}}{G_{F}^{\prime}}=\left(1-\left|U_{e 5}\right|^{2}\right)\left(1-\left|U_{\mu 5}\right|^{2}\right)=0.9987 \pm 0.0011
$$

resulting in an upper limit

$$
\left|U_{e 5} U_{\mu 5}\right|<0.0021
$$

at $99 \%$ CL. We mark this line as $\tau_{\mu}$.

Searches for lines in $\pi$ and $K$ meson decays: Measurements of $\pi, K \rightarrow \ell_{\alpha} n$ give important bounds on the mass and mixing of $n_{5}$ with $\nu_{\alpha}$. These are summarized in Figs. (2-4) of [24]. Bounds are given by null searches for peaks in the spectra of the leptonic products of these decays. For $n_{5}$ produced by the decay of a heavy parent particle $M$ of mass $m_{M}$ with a decay partner $\ell_{\alpha}$ of mass $m_{\alpha}$ we expect to see a monochromatic line in the lepton spectrum at $E_{\alpha}=\left(m_{M}^{2}+m_{\alpha}^{2}-m_{5}^{2}\right) / 2 m_{M}$. These lines are generically not found, and limits on $n_{5}$ mixing are based on the specifics of the given experiment.

For the electron neutrino sector, the decay $\pi \rightarrow e \nu$ [43] is strongest below $m_{\pi}$ and $K \rightarrow e \nu$ [44] is strongest between $m_{\pi}$ and $m_{K}$. For the muon neutrino sector, the important decay is $K \rightarrow \mu \nu[45]$. In the region $m_{5}>m_{\pi}-m_{\mu}$, muons cannot be produced in $\pi$ decay so there are no muon bounds in that range. Thus, line searches of $\pi$ decays do not provide strong constraints on the product $\left|U_{e 5} U_{\mu 5}\right|$ in the mass range $m_{5}>m_{\pi}-m_{\mu}$ since experiments cannot set any bounds on $U_{\mu 5}$ in this range. 
Decays of $n_{5}$ : If $n_{5}$ is heavy and can decay to SM products, these decays will be seen in dedicated searches such as, e.g., the PS191 [46], CHARM [47], and DELPHI [48] experiments. PS191 looked for the decay of a heavy neutrino through a variety of weak interaction channels; it is constraining from $\sim 1 \mathrm{MeV}^{3}$ to $138 \mathrm{MeV}$. CHARM searched for decays $n_{5} \rightarrow \ell^{+} \ell^{-} \nu$, where $\ell=e, \mu$, with constraints from 500 $\mathrm{MeV}$ to $2.8 \mathrm{GeV}$. DELPHI also looked for a wide variety of $n_{5}$ decays, and it provides limits from $2 \mathrm{GeV}$ to $90 \mathrm{GeV}$. We show these excluded regions as well as the limits from $n_{5}$ decays in dileptonic $K$ decays [38]. Note that, as pointed out previously [49], the PS191 and CHARM collaborations considered $n_{5}$ decays through charge-current channels only. When the necessary neutral-current contributions are added [24], the bounds are strengthened somewhat compared to the published results [49]. We provide bounds including both the charge- and neutral-current contributions.

Non-observation of $\mu \rightarrow e \gamma:$ For the decay $\mu \rightarrow e \gamma$, we have the standard result

$$
\operatorname{Br}(\mu \rightarrow e \gamma)=\frac{3 \alpha}{8 \pi}\left|\sum_{i} U_{e i} U_{\mu i}^{*} g\left(m_{i}\right)\right|^{2},
$$

where $g\left(m_{i}\right)$ is a kinematic factor given in [24]. This is not constraining below $\mathcal{O}(1$ $\mathrm{GeV}$ ) and by $300 \mathrm{GeV}$ the bound asymptotes to $\left|U_{e 5} U_{\mu 5}\right| \lesssim 5.25 \times 10^{-5}$ using the current measurement $\operatorname{Br}(\mu \rightarrow e \gamma) \leq 2.4 \times 10^{-12}$ [50]. At high mass, this is the most important constraint. In particular, measurements at the $Z$-pole are weaker than $\mu \rightarrow e \gamma$, so we do not show these bounds on our plots.

\subsubsection{Combined Bounds on $n_{5}$}

In Fig. 5.5, we show bounds on the product $\left|U_{e 5} U_{\mu 5}\right|$, which in the CP-conserving limit is the product that sets the value of $r$ in the appearance probability formula,

\footnotetext{
${ }^{3}$ The PS191 experiment did not publish limits for mixing angles above $10^{-4}$, so we extrapolate the bounds down to $m_{5}=2 m_{e}$ as a power law with $\propto \sqrt{m_{5}^{5}}$.
} 
Eq. (5.4). There are several model-independent bounds: as described above, BBN is most constraining below $\sim 1 \mathrm{MeV}$; there are universally constraining bounds for masses $0.1 \mathrm{MeV}<m_{5}<100 \mathrm{MeV}$ from SN1987A; the NuTeV oscillation search [16] rules out mixing angles $\left|U_{\mu 5} U_{e 5}\right|>1.3 \times 10^{-2}$ for the entire mass range; and for masses $m_{5}>64 \mathrm{GeV}$, the bounds from $\mu \rightarrow e \gamma$ are most stringent. In the range $100 \mathrm{MeV}$ $<m_{5}<64 \mathrm{GeV}$ the constraints bifurcate depending on whether $n_{5}$ decays to charged leptons or remains invisible on collider timescales.

Invisible decays: When $n_{5}$ remains invisible on collider timescales there are constraints from line searches, the pion branching fraction, and precision electroweak measurements of $G_{F}$. From $m_{\pi}-m_{\mu}<m_{5}<m_{\pi}$ the strongest bound is from the measured branching fraction of pion decays, $R_{\pi}$. For $m_{\pi}<m_{5}<m_{K}-m_{\pi}$ searches for leptonic lines in kaon decays are constraining for both $e$ and $\mu$ products. For $m_{\mu}<m_{5}$, comparing the values of $G_{F}$ from measurements of $\tau_{\mu}$ and the $W$ and $Z$ masses as described above gives tight constraints. We show these bounds in the left panel of Fig. 5.5.

Between the $K$ line searches and the $\mu \rightarrow e \gamma$ curve, where $387 \mathrm{MeV}<m_{5} \lesssim$ $10 \mathrm{GeV}$, the most constraining bounds on $n_{5}$ come from the precision electroweak measurements of $G_{F}$. Although this is the least constrained region, we find that these measurements still disfavor large values of $r$. Assuming no $\mathrm{CP}$ violation and taking $\left|U_{e 4} U_{\mu 4}\right|=0.023$, which is the smallest value of $\left|U_{e 4} U_{\mu 4}\right|$ for which $\left|U_{e 5} U_{\mu 5}\right|$ can take on arbitrarily low values in the MB and LSND region, we find that $r<1.09$.

Visible decays: In addition to the SN1987A and $\mu \rightarrow e \gamma$ bounds and the low mass constraints on BBN, we find that the direct searches at PS191, CHARM, and DELPHI are very constraining if $n_{5}$ decays to SM particles on detector timescales, and we also find that the BBN constraints can be extended to $m_{5} \simeq m_{\pi}$, as described 

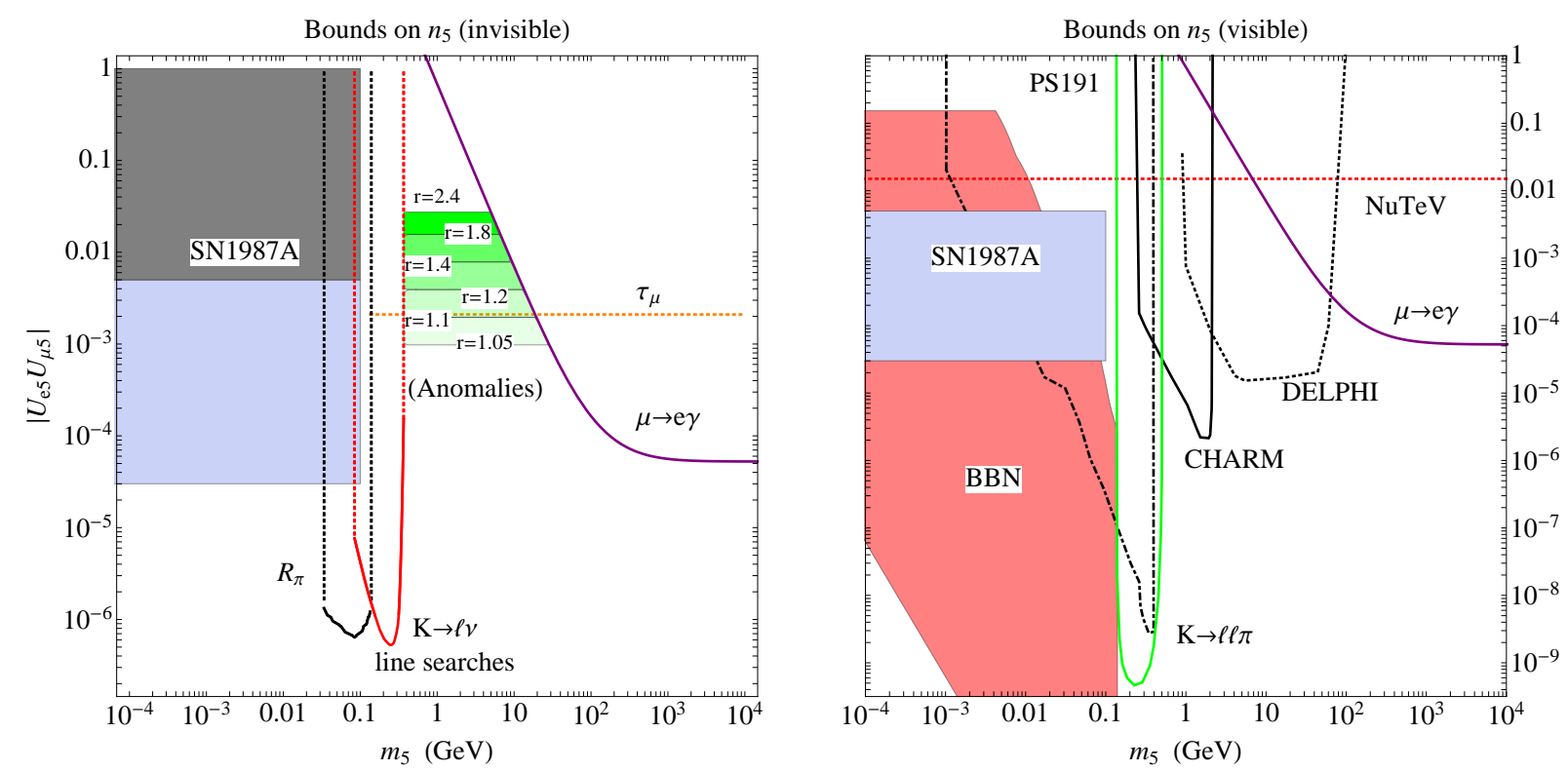

Figure 5.5: Exclusion regions from BBN [34] (right frame) and SN1987A [96] (both frames), as well as bounds from the $\mathrm{NuTeV}$ oscillation search [16] (red dotted, right frame), $R_{\pi}[37,38]$ (left frame), measurements of $\tau_{\mu}[38-$ 42] (left frame), collider and line searches [38,43-49] (both frames), and searches for $\mu \rightarrow e \gamma[50]$ (both frames). The left panel shows lines of constant values of $r$ from 1.05 to 2.4 (for the calculation of $r$, we assume no CP violation and take $\left|U_{e 4} U_{\mu 4}\right|=0.023$, as explained in the text). To avoid clutter, we avoid repeating the $\tau_{\mu}$ and $\mathrm{NuTeV}$ lines in both plots, although each is valid in both cases.

above. These give the most powerful constraints from $\lesssim 1 \mathrm{MeV}$ to $64 \mathrm{GeV}$. Above this range, the $\mu \rightarrow e \gamma$ constraints become powerful. These bounds are in the right panel of Fig. 5.5.

We see in Fig. 5.5 that the bounds are prohibitively strong if $n_{5}$ decays to SM products. We find that $n_{5}$ is phenomenologically more viable provided the decays of $n_{5}$ are invisible and the mass satisfies $387 \mathrm{MeV}<m_{5} \lesssim 10 \mathrm{GeV}$. However, when restricting the range of $\left|U_{e 5} U_{\mu 5}\right|$ from the muon lifetime, the LSND and MB results strongly favor $r \sim 1$. Furthermore, the combination of constraints from SN1987A and the 
muon lifetime restricts $\left|U_{e 5}\right|^{2}<0.004$ for $m_{5} \gtrsim 100 \mathrm{MeV}$, which seriously constrains the parameter space for solving the RAA and GA data, as indicated by Eq. (5.7).

In the next section we construct models of neutrino mass that naturally allow for invisible decays.

\subsection{Neutrino Models}

The $3+1+1$ scenario relies on the presence of a heavy neutrino with a substantial mixing with the light neutrinos. Within the standard see-saw scenario, with one active neutrino $\nu_{a}$ and one sterile neutrino $\nu_{m}$, this is not possible to achieve. The mass matrix

$$
\mathcal{M}=\left(\begin{array}{cc}
0 & m_{D} \\
m_{D} & M
\end{array}\right)
$$

connects the mixing to the mass hierarchy, so that a heavy sterile neutrino necessarily has a small mixing with the SM neutrino: $\theta \sim m_{D} / M$, which is small for a sizable neutrino hierarchy.

A large mass hierarchy and a large mixing can, however, be achieved for a Dirac sterile neutrino. Taking a single active neutrino $\nu_{a}$ and a sterile neutrino $\nu_{d}$ with Dirac partner $\bar{\nu}_{d}$, we can write a general mass matrix in the $\left(\nu_{a}, \nu_{d}, \bar{\nu}_{d}\right)$ basis as

$$
\mathcal{M}=\left(\begin{array}{ccc}
m_{1} & m_{D} & 0 \\
m_{D} & 0 & m_{5} \\
0 & m_{5} & 0
\end{array}\right)
$$

Defining $M^{2} \equiv m_{5}^{2}+m_{D}^{2}$ and expanding to second order in the small ratio $m_{1} / m_{5}$ we 
find the eigenvalues

$$
\begin{aligned}
& \lambda_{1}=M+\left(\frac{m_{D}^{2}}{2 M^{2}}\right) m_{1}+\left[\frac{m_{D}^{2}\left(m_{D}^{2}+4 m_{5}^{2}\right)}{8 M^{5}}\right] m_{1}^{2}+\ldots \\
& \lambda_{2}=-M+\left(\frac{m_{D}^{2}}{2 M^{2}}\right) m_{1}-\left[\frac{m_{D}^{2}\left(m_{D}^{2}+4 m_{5}^{2}\right)}{8 M^{5}}\right] m_{1}^{2}+\ldots \\
& \lambda_{3}=\frac{m_{5}^{2}}{M^{2}} m_{1}
\end{aligned}
$$

corresponding to the (unnormalized) eigenvectors

$$
K_{1}=\left(\begin{array}{c}
\frac{m_{D}}{M}\left(1+\frac{m_{1}}{M}\right) \\
1 \\
\frac{m_{5}}{M}
\end{array}\right) \quad K_{2}=\left(\begin{array}{c}
-\frac{m_{D}}{M}\left(1-\frac{m_{1}}{M}\right) \\
1 \\
-\frac{m_{5}}{M}
\end{array}\right) \quad K_{3}=\left(\begin{array}{c}
-\frac{M^{2}}{m_{1} m_{D}} \\
1 \\
\frac{M^{2}}{m_{1} m_{5}}
\end{array}\right)
$$

$K_{1}$ and $K_{2}$ correspond to the components of the mostly sterile fifth mass eigenstate $n_{5}$, whereas $K_{3}$ corresponds to a mostly active light state. The mixing between $n_{5}$ and the light state is controlled by $m_{D} / M$. This ratio need not be very small since the mass of the light neutrino is fixed independently by $m_{1}$. The small mixing scenario is recovered in the limit $m_{D} \ll m_{5}$, which corresponds to $m_{5} \rightarrow M$, while maximal mixing corresponds to the limit $m_{D} \rightarrow m_{5}$.

This type of scenario can be extended to encompass the fourth neutrino, as well as the needed invisible decays of $n_{5}$. Consider adding to the Lagrangian a term

$$
\mathcal{L}_{\phi}=\lambda \phi \nu_{d} \nu_{m}+\lambda^{\prime} \phi \nu_{m}^{2}
$$

Neglecting Majorana mass terms for illustration, we find that a mass matrix in the 
$\left(\nu_{a}, \nu_{d}, \bar{\nu}_{d}, \nu_{m}\right)$ basis with the desired phenomenology is given by

$$
\mathcal{M}=\left(\begin{array}{cccc}
0 & m_{D} & 0 & 0 \\
m_{D} & 0 & m_{5} & m_{\phi} \\
0 & m_{5} & 0 & 0 \\
0 & m_{\phi} & 0 & 0
\end{array}\right)
$$

where $m_{\phi}=\lambda v_{\phi}$. This matrix has two zero eigenvalues, with the other two set

by $\pm \sqrt{m_{\phi}^{2}+m_{D}^{2}+m_{5}^{2}}$. In a hierarchy where $m_{\phi} \ll m_{D} \ll m_{5}$, the massive states are mostly $\nu_{d}$ and their mixing with the active neutrino is controlled by $m_{D} / m_{5}$. The massless states are predominantly composed of $\nu_{a}$ and $\nu_{m}$, and their mixing is controlled by $m_{\phi} / m_{D}$. Of course, these masses should not exactly vanish, and the masses can be lifted from being zero by appropriately small Majorana mass terms.

The new state $\phi$ allows both for large $\nu_{d}-\nu_{a}$ mixing and for invisible decays of $\nu_{d}$ (via $\nu_{d} \rightarrow \phi \nu_{m}$ with subsequent decays $\phi \rightarrow \nu_{m} \nu_{m}$ ). This decay, with width $\Gamma_{\phi} \sim \frac{1}{16 \pi} \lambda^{2} m_{5}$, should be compared to the decay derived from mixing with active states, which scales as $\Gamma_{S M} \sim \frac{1}{16 \pi} \theta_{\mu, e}^{2} g_{Z}^{2}\left(\frac{m_{5}}{m_{Z}}\right)^{4} m_{5}$. Since $m_{5}$ is in the GeV range, the SM decay channel is naturally suppressed with respect to the invisible decay.

\subsection{Conclusions}

We have studied fits to the LSND and MB experiments within the context of $3+1$ and $3+1+1[1]$ scenarios. Compared to the $3+1$ scenario, the $3+1+1$ framework posits that the presence of an additional heavy neutrino which is not directly probed by most disappearance experiments lifts some of the constraints of the null disappearance experiments. However, using the new $2011 \mathrm{MB} \bar{\nu}$ data, we find there is still significant tension between positive and the null results, even with the additional very heavy neutrino. We went on to explore the phenomenology of the massive neutrino that 
appears in the $3+1+1$ scenario, and we found that a heavy mostly sterile neutrino could be consistent with a variety of cosmological and collider constraints if the sterile neutrino has a mass around a $\mathrm{GeV}$ and does not couple primarily to the SM. We also showed that in the face of BBN, direct search, and precision electroweak bounds, even a heavy state that decays invisibly might not be suitable for reconciling the anomalous measurements of $\nu_{e}$ fluxes made by gallium and reactor experiments.

If the larger mixing angle required by the $2011 \bar{\nu}$ data for the LSND and MB anomaly persists, other types of scenarios will be required in order to obtain a consistent global explanation of the neutrino oscillation data. One possibility, which was explored in [52], is to make use of medium dependent neutrino masses [53]. In this case, Bugey (whose oscillations would mostly occur through air) would be weakened relative to LSND and MB (whose oscillations mostly occur through earth), and a wide swath of parameter space would remain. We leave this possibility for future consideration.

Acknowledgments: We thank Ann Nelson and Tomer Volansky for discussions. We would also like to thank Enrique Fernandez Martinez, Oleg Ruchayskiy, and Jinrui Huang for pointing out some additional bounds relevant to the parameter space of Fig. 5.5. The work of EK is supported in part by a grant from the Israel Science Foundation. The work of KMZ and SDM is supported by NSF CAREER PHY 1049896, and NASA Astrophysics Theory Program Grant No. NNX11AI17G. 


\section{Bibliography}

[1] A. E. Nelson, Phys. Rev. D 84, 053001 (2011) [arXiv:1010.3970 [hep-ph]].

[2] A. Aguilar et al. [LSND Collaboration], Phys. Rev. D 64, 112007 (2001) [hepex/0104049].

[3] A. A. Aguilar-Arevalo et al. [The MiniBooNE Collaboration], Phys. Rev. Lett. 98, 231801 (2007) [arXiv:0704.1500 [hep-ex]].

[4] A. A. Aguilar-Arevalo et al. [MiniBooNE Collaboration], Phys. Rev. Lett. 102, 101802 (2009) [arXiv:0812.2243 [hep-ex]].

[5] A. A. Aguilar-Arevalo et al. [The MiniBooNE Collaboration], Phys. Rev. Lett. 105, 181801 (2010) [arXiv:1007.1150 [hep-ex]].

[6] J. Kopp, M. Maltoni and T. Schwetz, Phys. Rev. Lett. 107, 091801 (2011) [arXiv:1103.4570 [hep-ph]].

[7] M. Maltoni and T. Schwetz, Phys. Rev. D 76, 093005 (2007) [arXiv:0705.0107 [hep-ph]].

[8] Z. Djurcic [MiniBooNE Collaboration], arXiv:1201.1519 [hep-ex]. W. Huelsnitz, SUSY (2011).

http://www-boone.fnal.gov/slides-talks/conf-talk/huelsnitz/ huelsnitz_SUSY_2011.pdf

[9] J. Fan and P. Langacker, JHEP 1204, 083 (2012) [arXiv:1201.6662 [hep-ph]].

[10] C. Giunti and M. Laveder, Phys. Lett. B 706, 200 (2011) [arXiv:1111.1069 [hep$\mathrm{ph}]]$.

[11] B. Bhattacharya, A. M. Thalapillil and C. E. M. Wagner, Phys. Rev. D 85, 073004 (2012) [arXiv:1111.4225 [hep-ph]]. 
[12] B. Armbruster et al. [KARMEN Collaboration], Phys. Rev. D 65, 112001 (2002) [hep-ex/0203021].

[13] L. Borodovsky, C. Y. Chi, Y. Ho, N. Kondakis, W. -Y. Lee, J. Mechalakos, B. Rubin and R. Seto et al., Phys. Rev. Lett. 68, 274 (1992).

[14] P. Astier et al. [NOMAD Collaboration], Phys. Lett. B 570, 19 (2003) [hepex/0306037].

[15] A. Romosan et al. [CCFR/NuTeV Collaboration], Phys. Rev. Lett. 78, 2912 (1997) [hep-ex/9611013].

[16] S. Avvakumov, T. Adams, A. Alton, L. de Barbaro, P. de Barbaro, R. H. Bernstein, A. Bodek and T. Bolton et al., Phys. Rev. Lett. 89, 011804 (2002) [hepex/0203018].

[17] G. Mention, M. Fechner, T. .Lasserre, T. .A. Mueller, D. Lhuillier, M. Cribier and A. Letourneau, Phys. Rev. D 83, 073006 (2011) [arXiv:1101.2755 [hep-ex]].

[18] Y. Declais et al., Nucl. Phys. B 434, 503 (1995).

[19] F. Dydak, G. J. Feldman, C. Guyot, J. P. Merlo, H. J. Meyer, J. Rothberg, J. Steinberger and H. Taureg et al., Phys. Lett. B 134, 281 (1984).

[20] I. E. Stockdale et al., In the Proceedings of 1984 Meeting of the Division of Particles and Fields of the APS, Santa Fe, New Mexico, 31 Oct - 3 Nov 1984, pp 258 .

[21] R. Wendell et al. [Super-Kamiokande Collaboration], Phys. Rev. D 81, 092004 (2010) [arXiv:1002.3471 [hep-ex]].

[22] M. Maltoni and T. Schwetz, Phys. Rev. D 68, 033020 (2003) [hep-ph/0304176]. 
[23] P. Langacker and D. London, Phys. Rev. D 38, 886 (1988). P. Langacker and D. London, Phys. Rev. D 38, 907 (1988).

[24] A. Atre, T. Han, S. Pascoli, B. Zhang, JHEP 0905, 030 (2009). [arXiv:0901.3589 [hep-ph]].

[25] P. Huber, Phys. Rev. C 84, 024617 (2011) [Erratum-ibid. C 85, 029901 (2012)] [arXiv:1106.0687 [hep-ph]].

[26] J. N. Abdurashitov et al. [SAGE Collaboration], Phys. Rev. C 80, 015807 (2009) [arXiv:0901.2200 [nucl-ex]]. F. Kaether, W. Hampel, G. Heusser, J. Kiko and T. Kirsten, Phys. Lett. B 685, 47 (2010) [arXiv:1001.2731 [hep-ex]].

[27] D. Frekers, H. Ejiri, H. Akimune, T. Adachi, B. Bilgier, B. A. Brown, B. T. Cleveland and H. Fujita et al., Phys. Lett. B 706, 134 (2011).

[28] B. E. Bodmann et al. [KARMEN. Collaboration], Phys. Lett. B 332, 251 (1994). L. B. Auerbach et al. [LSND Collaboration], Phys. Rev. C 64, 065501 (2001) [hep-ex/0105068].

[29] M. Fukugita, Y. Kohyama and K. Kubodera, Phys. Lett. B 212, 139 (1988).

[30] J. M. Conrad and M. H. Shaevitz, Phys. Rev. D 85, 013017 (2012) [arXiv:1106.5552 [hep-ex]].

[31] G. Mangano and P. D. Serpico, Phys. Lett. B 701, 296 (2011) [arXiv:1103.1261 [astro-ph.CO]].

[32] R. Foot and R. R. Volkas, Phys. Rev. Lett. 75, 4350 (1995) [hep-ph/9508275]. Y. -Z. Chu and M. Cirelli, Phys. Rev. D 74, 085015 (2006) [astro-ph/0608206]. K. Abazajian, N. F. Bell, G. M. Fuller and Y. Y. Y. Wong, Phys. Rev. D 72, 063004 (2005) [astro-ph/0410175]. 
[33] A. D. Dolgov and F. L. Villante, Nucl. Phys. B 679, 261 (2004) [hep-ph/0308083].

[34] O. Ruchayskiy and A. Ivashko, arXiv:1202.2841 [hep-ph].

[35] K. Kainulainen, J. Maalampi and J. T. Peltoniemi, Nucl. Phys. B 358, 435 (1991).

[36] G. G. Raffelt, M. T. .Keil, R. Buras, H. -T. Janka and M. Rampp, astro$\mathrm{ph} / 0303226$.

[37] V. Cirigliano, I. Rosell, Phys. Rev. Lett. 99, 231801 (2007). [arXiv:0707.3439 [hep-ph]].

[38] K. Nakamura et al. (Particle Data Group), J. Phys. G 37, 075021 (2010)

[39] A. Barczyk et al. [FAST Collaboration], Phys. Lett. B 663, 172 (2008) [arXiv:0707.3904 [hep-ex]].

[40] C. Biggio, M. Blennow and E. Fernandez-Martinez, JHEP 0908, 090 (2009) [arXiv:0907.0097 [hep-ph]].

[41] Tevatron Electroweak Working Group, f. t. C. Collaboration and D. Collaboration, arXiv:1204.0042 [hep-ex].

[42] [ALEPH and DELPHI and L3 and OPAL and SLD and LEP Electroweak Working Group and SLD Electroweak Group and SLD Heavy Flavour Group Collaborations], Phys. Rept. 427, 257 (2006) [hep-ex/0509008].

[43] D. I. Britton, S. Ahmad, D. A. Bryman, R. A. Burnham, E. T. H. Clifford, P. Kitching, Y. Kuno and J. A. Macdonald et al., Phys. Rev. D 46, 885 (1992).

[44] T. Yamazaki, T. Ishikawa, Y. Akiba, M. Iwasaki, K. H. Tanaka, S. Ohtake, H. Tamura and M. Nakajima et al., Conf. Proc. C 840719, 262 (1984). 
[45] R. S. Hayano, T. Taniguchi, T. Yamanaka, T. Tanimori, R. Enomoto, A. Ishibashi, T. Ishikawa and S. Sato et al., Phys. Rev. Lett. 49, 1305 (1982).

[46] G. Bernardi, G. Carugno, J. Chauveau, F. Dicarlo, M. Dris, J. Dumarchez, M. Ferro-Luzzi and J. -M. Levy et al., Phys. Lett. B 203, 332 (1988).

[47] F. Bergsma et al. [CHARM Collaboration], Phys. Lett. B 166, 473 (1986).

[48] P. Abreu et al. [DELPHI Collaboration], Z. Phys. C 74, 57 (1997) [Erratum-ibid. C 75, $580(1997)]$.

[49] A. Kusenko, S. Pascoli and D. Semikoz, JHEP 0511, 028 (2005) [hep$\mathrm{ph} / 0405198]$.

O. Ruchayskiy and A. Ivashko, JHEP 1206, 100 (2012) [arXiv:1112.3319 [hep$\mathrm{ph}]]$.

[50] J. Adam et al. [ MEG Collaboration ], Phys. Rev. Lett. 107, 171801 (2011). [arXiv:1107.5547 [hep-ex]].

[51] F. del Aguila, J. de Blas and M. Perez-Victoria, Phys. Rev. D 78, 013010 (2008) [arXiv:0803.4008 [hep-ph]].

[52] K. M. Zurek, JHEP 0410, 058 (2004) [hep-ph/0405141].

[53] D. B. Kaplan, A. E. Nelson and N. Weiner, Phys. Rev. Lett. 93, 091801 (2004) [hep-ph/0401099].

[54] S. Abe et al. [KamLAND Collaboration], Phys. Rev. Lett. 100, 221803 (2008) [arXiv:0801.4589 [hep-ex]].

[55] C. Giunti and M. Laveder, Phys. Rev. C 83, 065504 (2011) [arXiv:1006.3244 [hep-ph]]. 
[56] G. J. Feldman and R. D. Cousins, Phys. Rev. D 57, 3873 (1998) [physics/9711021 [physics.data-an]].

[57] S. Antusch, C. Biggio, E. Fernandez-Martinez, M. B. Gavela and J. Lopez-Pavon, JHEP 0610, 084 (2006) [hep-ph/0607020]. 


\section{CHAPTER VI}

\section{Constraining Light Dark Matter with Diffuse X-Ray and Gamma-Ray Observations}

We present constraints on decaying and annihilating dark matter (DM) in the $4 \mathrm{keV}$ to $10 \mathrm{GeV}$ mass range, using published results from the satellites HEAO-1, INTEGRAL, COMPTEL, EGRET, and the Fermi Gamma-ray Space Telescope. We derive analytic expressions for the gamma-ray spectra from various DM decay modes, and find lifetime constraints in the range $10^{24}-10^{28} \mathrm{sec}$, depending on the DM mass and decay mode. We map these constraints onto the parameter space for a variety of models, including a hidden photino that is part of a kinetically mixed hidden sector, a gravitino with R-parity violating decays, a sterile neutrino, DM with a dipole moment, and a dark pion. The indirect constraints on sterileneutrino and hidden-photino DM are found to be more powerful than other experimental or astrophysical probes in some parts of parameter space. While our focus is on decaying DM, we also present constraints on

Written in collaboration with Rouven Essig, Eric Kuflik, Tomer Volansky, and K. M. Zurek. Originally published as Constraining Light Dark Matter with Diffuse X-Ray and Gamma-Ray Observations, JHEP 1311, 193 (2013). arXiv:1309.4091 [hep-ph], MCTP-13-27, FERMILAB-PUB-13377-A-T. 
DM annihilation to electron-positron pairs. We find that if the annihilation is $p$-wave suppressed, the galactic diffuse constraints are, depending on the DM mass and velocity at recombination, more powerful than the constraints from the Cosmic Microwave Background.

\subsection{Introduction}

A wide variety of precision astrophysical and cosmological observations have corroborated the existence of dark matter (DM), without providing any conclusive indications of its nature or its non-gravitational couplings to the Standard Model (SM). For the past 30 years, a broad experimental program has attempted to uncover the DM properties. However, the vast majority of the existing experiments search either for Weakly Interacting Massive Particles (WIMPs) or for axions, overlooking other theoretically viable and motivated possibilities. One interesting possibility is light dark matter (LDM) in the $\mathrm{keV}$ to $10 \mathrm{GeV}$ mass range. In this paper, we focus on such DM and study constraints from existing indirect searches.

A large class of models can accommodate DM with sub-GeV masses, see, e.g., [19]. Such DM can be probed at colliders [10-15], at direct detection experiments $[9,16$, 17], and at proton- and electron-beam dumps [18-23]. Constraints from the Cosmic Microwave Background (CMB) already limit the s-wave DM annihilation cross section to SM matter to be below that of a thermal WIMP, for DM masses below $\sim 7 \mathrm{GeV}[24-$ $27]$.

While DM decays are less constrained by early Universe cosmology, stringent constraints can be placed on decaying DM from observations of the galactic and extra-galactic diffuse X-ray or gamma-ray background. The lifetime of Weak-scale DM is constrained from observations with the Fermi Large Area Telescope (Fermi LAT) to $\tau \gtrsim 10^{26}$ sec [28-32], many orders of magnitude larger than the age of the Universe. For DM below $\mathcal{O}(100 \mathrm{MeV})$, the usual gamma ray constraints from the 
Fermi LAT do not apply, although the instruments on several other satellites (listed in Table 6.1 below) are sensitive to photons with energies well below a $\mathrm{GeV}$. The available data cover a photon energy range from 10 's of $\mathrm{GeV}$ down to a few $\mathrm{keV}$, providing the possibility of exploring a much broader range of DM candidates than WIMPs. Indeed, some of these data have already been utilized to constrain LDM, see, e.g., $[18,33-42,44]$. Sterile neutrinos with a mass $\sim \mathcal{O}(1-10 \mathrm{KeV})$ are a particularly popular candidate and their constraints have been explored in, e.g., [36,38-40,45-51, 51-57]. Below a few keV, thermal DM candidates become too warm to adequately explain the formation of structure in the Universe, so that such candidates necessarily have a mass above the lower energy bound accessible by these satellite experiments.

The goal of this paper is to derive constraints on light DM candidates in the $\mathrm{keV}$ to $10 \mathrm{GeV}$ mass range, using the diffuse photon spectra data listed in Table 6.1. We update and extend several results in the literature. Taking a largely model-independent approach, we discuss a wide range of DM decay topologies. We consider photons that are produced directly in the decay or from final state radiation (FSR) of charged particles that are produced in two- or three-body decays. We map our results onto several known LDM models, and show limits on the corresponding model parameter space. For example, we consider constraints on a kinetically mixed supersymmetric hidden sector (with the hidden photino decaying to $\tilde{G} \gamma$ or $\tilde{G} e^{+} e^{-}$, with $\tilde{G}$ the gravitino) and a sterile neutrino (with the sterile neutrino decaying to a neutrino and a photon). While the constraints we derive are robust, they are based on published data. Consequently, they can easily be improved by optimizing the search regions and taking better account of the signal and background fitting.

While our focus is on decaying DM, we also consider annihilating DM. A thermal relic with a $p$-wave (or velocity suppressed) annihilation cross section is less constrained from CMB data than s-wave annihilation, since DM is cold at the CMB epoch. For this case, we find that the limits from the diffuse background can be more 


\begin{tabular}{|c|c|c|c|c|c|c|c|c|c|}
\hline Experiment & $E_{\min }$ & $E_{\max }$ & $\Omega$ & $\begin{array}{l}J_{D(A)}^{\mathrm{NFW}} \\
\end{array}$ & $J_{D(A)}^{\text {Moore }}$ & $J_{D(A)}^{\text {IsoT }}$ & $J_{D(A)}^{\operatorname{Ein}, 0.17}$ & $J_{D(A)}^{\text {Ein, } 0.12}$ & $J_{D(A)}^{\operatorname{Ein}, 0.20}$ \\
\hline HEAO-1 [63] & $4 \mathrm{KeV}$ & $30 \mathrm{KeV}$ & $\begin{array}{l}58 \leq \ell \leq 109^{\circ} \cup \\
238 \leq \ell \leq 289^{\circ}, \\
20^{\circ} \leq|b| \leq 90^{\circ}\end{array}$ & $\begin{array}{c}3.88 \\
(2.16)\end{array}$ & $\begin{array}{c}4.06 \\
(2.22)\end{array}$ & $\begin{array}{c}4.33 \\
(2.24)\end{array}$ & $\begin{array}{c}3.79 \\
(2.09)\end{array}$ & $\begin{array}{c}3.76 \\
(2.05)\end{array}$ & $\begin{array}{c}3.8 \\
(2.11)\end{array}$ \\
\hline INTEGRAL [64] & $20 \mathrm{KeV}$ & $1 \mathrm{MeV}$ & $\begin{array}{l}|\ell| \leq 30^{\circ} \\
|b| \leq 15^{\circ}\end{array}$ & $\begin{array}{c}3.65 \\
(18.4)\end{array}$ & $\begin{array}{l}3.80 \\
(24.4)\end{array}$ & $\begin{array}{c}2.77 \\
(5.08)\end{array}$ & $\begin{array}{c}4.20 \\
(30.9)\end{array}$ & $\begin{array}{c}4.73 \\
(59.9)\end{array}$ & $\begin{array}{c}3.95 \\
(23.2)\end{array}$ \\
\hline COMPTEL [65] & $1 \mathrm{MeV}$ & $15 \mathrm{MeV}$ & $\begin{array}{l}|\ell| \leq 60^{\circ} \\
|b| \leq 20^{\circ}\end{array}$ & $\begin{array}{c}6.82 \\
(23.1)\end{array}$ & $\begin{array}{c}7.03 \\
(29.1)\end{array}$ & $\begin{array}{c}5.91 \\
(8.69) \\
\end{array}$ & $\begin{array}{c}7.48 \\
(36.4) \\
\end{array}$ & $\begin{array}{c}8.10 \\
(66.0)\end{array}$ & $\begin{array}{c}7.19 \\
(28.3) \\
\end{array}$ \\
\hline EGRET [66] & $20 \mathrm{MeV}$ & $6 \mathrm{GeV}$ & $\begin{array}{c}0 \leq \ell \leq 360^{\circ} \\
20^{\circ} \leq|b| \leq 60^{\circ}\end{array}$ & $\begin{array}{c}13.0 \\
(10.9)\end{array}$ & $\begin{array}{c}13.5 \\
(11.0)\end{array}$ & $\begin{array}{c}14.0 \\
(10.1)\end{array}$ & $\begin{array}{c}12.9 \\
(11.5)\end{array}$ & $\begin{array}{c}13.0 \\
(12.0)\end{array}$ & $\begin{array}{c}12.9 \\
(11.3)\end{array}$ \\
\hline Fermi [67] & $200 \mathrm{MeV}$ & $10 \mathrm{GeV}$ & $\begin{array}{c}0 \leq \ell \leq 360^{\circ} \\
8^{\circ} \leq \mid \overline{b \mid} \leq 90^{\circ}\end{array}$ & $\begin{array}{c}21.9 \\
(22.0)\end{array}$ & $\begin{array}{l}22.8 \\
(22.5)\end{array}$ & $\begin{array}{c}23.3 \\
(17.9)\end{array}$ & $\begin{array}{c}22.0 \\
(25.4)\end{array}$ & $\begin{array}{c}22.3 \\
(28.5)\end{array}$ & $\begin{array}{l}21.9 \\
(24.0)\end{array}$ \\
\hline
\end{tabular}

Table 6.1: Energy ranges, solid angles, and values of $J_{D}\left(J_{A}\right)$ for various DM density profiles. The NFW profile is taken from [58,59], the Moore profile from [60], and the cored isothermal profile can be found in [61]. The profiles "Ein, $\alpha "$ are Einasto profiles [62] with slope parameter $\alpha$.

constraining than the CMB.

The outline of the paper is as follows. In Sec. 6.2, we review both the expected signals resulting from DM decays and annihilations as well as the relevant gamma-ray and X-ray observatories (HEAO-1 [63], INTEGRAL [64], COMPTEL [65], EGRET [66], Fermi [67]). We further discuss our methods for placing the limits on such DM. In Sec. 6.3, we discuss models of decaying light DM such as decaying gravitinos, sterile neutrinos, and hidden photinos. For each model we map the lifetime constraints onto constraints of the model parameter space. In Sec. 6.4, we take a model-independent approach and constrain the lifetime for various decay topologies. Sec. 6.5 is devoted to constraints on the annihilation cross-section of light DM to electron-positron pairs. We conclude in Sec. 6.6.

\subsection{Constraining Light Dark Matter with Diffuse Photons}

In this section, we discuss the data and the statistical methodwe use to place constraints on decaying and annihilating LDM. We begin with a brief review of the expected signal rate. 


\subsubsection{Flux from Dark Matter Decays and Annihilations}

Given a DM annihilation or decay spectrum, $d N_{\gamma} / d E_{\gamma}$, and a galactic DM density profile, $\rho(r)$, the galactic contribution to the differential photon flux per unit energy is given by,

$$
\frac{d \Phi_{\gamma, \mathrm{G}}}{d E}=\frac{1}{2^{\alpha-1}} \frac{r_{\odot}}{4 \pi} \frac{\rho_{\odot}}{m_{\mathrm{DM}}} \Gamma_{D, A} \frac{d N_{\gamma}}{d E} J_{D, A}
$$

Here $r_{\odot} \simeq 8.5 \mathrm{kpc}$ is the Sun's distance from the Galactic center, $\rho_{\odot}=0.3 \mathrm{GeV} / \mathrm{cm}^{3}$ is the local DM density, $\alpha=1$ (2) for DM decays (annihilations), $\Gamma_{D}$ is the decay rate, $\Gamma_{A}=\left(\rho_{\odot} / m_{\mathrm{DM}}\right)\langle\sigma v\rangle$ is the thermally averaged annihilation rate, and

$$
J_{D, A}=\int_{\text {l.o.s. }} \frac{d s}{r_{\odot}}\left[\frac{\rho(s)}{\rho_{\odot}}\right]^{\alpha} d \Omega
$$

is a dimensionless quantity that describes the density of decays or annihilations along the line-of-sight (l.o.s.) and over the solid angle $\Omega$. We will present results assuming $\rho(s)$ follows the NFW DM density profile $[58,59]$, but in Table 6.1 we also list values of $J_{D, A}$ for other DM density profiles for each experimental survey region. Our results can thus be easily rescaled. Note that the choice of $\rho(s)$ becomes less important for survey regions farther from the galactic plane and also less important for decaying compared to annihilating DM.

In addition to the contribution to the photon flux from DM decays in the Milky Way halo, there is a contribution arising from the smooth distribution of DM throughout the whole Universe (see, e.g., $[37,45-47]$ ). A photon produced at redshift $z$ that is detected with energy $E$ was emitted with energy $E(z)=E(1+z)$. Such a photon was emitted at a comoving distance, $\chi(z)$, with

$$
\frac{d \chi(z)}{d z}=\frac{1}{(1+z)^{3 / 2}} \frac{1}{a_{0} H_{0} \sqrt{\Omega_{m}\left(1+\kappa(1+z)^{-3}\right)}}
$$

where $\kappa=\Omega_{\Lambda} / \Omega_{m} \sim 3$ and a flat Universe, $\Omega_{m}+\Omega_{\Lambda}=1$, is assumed. The extra- 
galactic photon spectrum arising from DM decays at redshift $z$ is given by $d N / d E(z)$, so that the measured flux is

$$
\frac{d^{2} \Phi_{\gamma, E G}}{d \Omega d E}=\frac{1}{4 \pi} \frac{\Gamma \Omega_{\mathrm{DM}} \rho_{c}}{m_{\mathrm{DM}} a_{0} H_{0} \sqrt{\Omega_{m}}} \int_{0}^{\infty} d z \frac{d N}{d E(z)} \frac{1}{(1+z)^{3 / 2}} \frac{1}{\sqrt{1+\kappa(1+z)^{-3}}}
$$

Because the photon flux from DM decays scales linearly with the DM density, this contribution is not very model dependent. For $d N_{\gamma} / d E(z)=\delta\left(E(z)-m_{\mathrm{DM}} / 2\right)$, this reduces to the case that is usually considered, namely DM decaying to a redshifted monochromatic gamma-ray line,

$$
\frac{d^{2} \Phi_{\gamma, E G}}{d \Omega d E}=\frac{1}{4 \pi} \frac{\Gamma \Omega_{\mathrm{DM}} \rho_{c}}{m_{\mathrm{DM}} H_{0} \sqrt{\Omega_{m}}}\left(\frac{2}{m_{\mathrm{DM}}}\right) \sqrt{\frac{2 E}{m_{\mathrm{DM}}}} \frac{1}{\sqrt{1+\kappa\left(2 E / m_{\mathrm{DM}}\right)^{3}}} .
$$

This effect implies that the spectral shape of a photon "line" from DM decays is smeared to receive contributions from a continuous range of energies.

In principle, similar extragalactic contributions exist for the annihilating DM case. However, the smooth part of extragalactic DM annihilation is subdominant compared to the galactic contribution and may be safely ignored. On the other hand, extragalactic annihilations resulting from DM substructure at low redshift may contribute a significant amount to the photon flux since it scales as the square of the DM density [20]. Since this contribution is not well known [69], we conservatively omit it from our analysis below.

For DM decay or annihilation to final states that include electrons or positrons, there are other potentially important contributions to the diffuse photon flux. The electrons and positrons can inverse Compton scatter (ICS) starlight, infrared, or CMB photons, or produce synchrotron radiation. The precise contribution to the diffuse flux, however, is model dependent and requires detailed knowledge of the galactic and extragalactic magnetic fields as well as the diffusion properties of the electrons in our Galaxy. In order to present conservative bounds and to avoid significant systematic 
uncertainties, we do not include these contributions.

When stable charged particles (like electrons) appear as decay or annihilation products, photons will be emitted through final state radiation (FSR). We use the Altarelli-Parisi splitting function,

$$
\frac{d \Gamma_{\mathrm{FSR}}}{d E_{\gamma}}=\frac{\alpha_{\mathrm{EM}} \Gamma_{D, A}}{2 \pi} \ln \left(Q / m_{f}^{2}\right) \int \frac{1+\left(1-E_{\gamma} / E_{f}\right)^{2}}{E_{\gamma}} \frac{d N}{d E_{f}} d E_{f},
$$

to estimate the photon spectrum, where $Q$ is the square of the momentum imparted to the photon, $\alpha_{\mathrm{EM}} \simeq 1 / 137$, and $d N / d E_{f}$ is the differential rate of decay or annihilation to the final state particle $f$. For multiple charged-particles in the final state, we sum over the contributions.

\subsubsection{Data}

We place constraints on LDM using the data summarized in Tab. 6.1 and shown in Fig. 6.1. We emphasize that none of the datasets have been optimized for LDM searches. It is therefore likely that significantly stronger constraints may be achieved with dedicated analyses.

As mentioned above, we assume an NFW profile in all cases, but the results can easily be rescaled for other profiles using the information in Tab. 6.1. For the innergalaxy data from INTEGRAL or COMPTEL, the bounds from decaying DM can be adjusted by up to $\mathcal{O}(30 \%)$; using high-latitude data, the difference is typically less than $\mathcal{O}(10 \%)$. In contrast, the expected photon flux from DM annihilations near the galactic center can change by up to an order of magnitude for different choices of the density profile.

For our analysis we use the following datasets:

- HEAO-1. We use data from observations of 3-50 keV photons made with the A2 High-Energy Detector on HEAO-1 [63]. Other datasets from the experiment 
Data

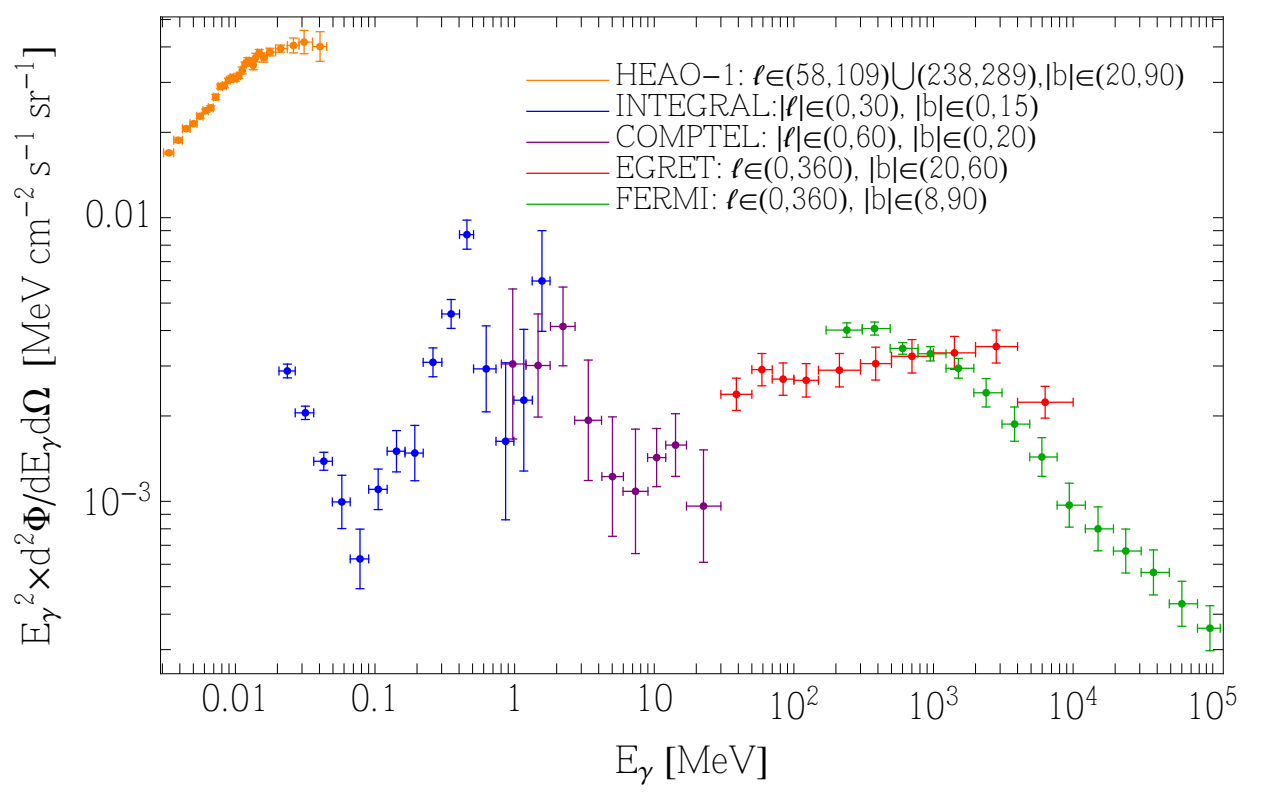

Figure 6.1: The collected normalized dataset of photon fluxes used to place constraints on decaying and annihilating DM in this paper. Data from HEAO-1 [63] (orange), INTEGRAL [64] (green), COMPTEL [65] (blue), EGRET [66] (red), and Fermi [67] (yellow) are shown. All error bars are statistical, except for the EGRET and Fermi datasets, where the dominant systematic uncertainties are shown. We omit the INTEGRAL 511 $\mathrm{keV}$ line both in this figure and in our analysis. Note that the various datasets span different regions of the sky and should therefore not be compared with each other; they appear together on this plot only for convenience.

are significantly weaker than those from the INTEGRAL experiment discussed below. To avoid point source contamination, the observations come from regions of the sky $20^{\circ}$ above the galactic plane. As is clear from Tab. 6.1, the constraints from this sky region are not very sensitive to the DM density profile.

- INTEGRAL. We use data from observations of $20 \mathrm{keV}$ to $2 \mathrm{MeV}$ photons from the region $|\ell|<30^{\circ}$ and $|b|<15^{\circ}$ obtained with the SPI instrument onboard INTEGRAL [64]. The quantity $J$ changes by up to $\mathcal{O}(30 \%)$ in the decaying case, for different choices of density profile. The excellent energy resolution allows us to remove the well-resolved $511 \mathrm{keV}$ line in our analysis. 
- COMPTEL. We use the COMPTEL data from [65]. These observations are obtained by averaging over the sky at latitudes $|\ell| \leq 60^{\circ}$ and $|b|<20^{\circ}$. Compared to the INTEGRAL region of interest, the model predictions are about half as sensitive to the density profile at these galactic latitudes. We find an $\mathcal{O}(20 \%)$ uncertainty for DM decay bounds due to the DM density profile.

- EGRET. We use the data shown in panel E of Fig. 2 in [66], which lies in the $20 \mathrm{MeV}$ to $10 \mathrm{GeV}$ range at intermediate latitudes, $0 \leq \ell \leq 360^{\circ}, 20^{\circ} \leq|b| \leq$ $60^{\circ}$. Our results are sensitive only at the few-percent level to the DM density profile.

- Fermi. We use data from the upper panel of Fig. 12 of [67], with $0<\ell<360^{\circ}$ and $8^{\circ}<|b|<90^{\circ}$, between $200 \mathrm{MeV}-10 \mathrm{GeV}$. We choose these latitudes to enhance the signal to background ratio while minimizing the uncertainty in the DM profile. The resulting decay bounds are only $\mathcal{O}(5 \%)$ sensitive to varying the DM density profile.

\subsubsection{Statistical Methodology}

Our goal is to obtain robust, conservative bounds using the above data sets. We do this by requiring that the predicted count from the DM signal in each bin does not exceed the observed central value plus twice the error bar. In all cases we use the statistical uncertainties, except for EGRET and Fermi where we take the dominant systematic uncertainties. These bounds could be significantly strengthened with dedicated searches in the future and by including fits to different astrophysical background components, e.g., from astrophysical ICS. In Appendix ??, we show the improvement that could be obtained with a goodness-of-fit test that assumes knowledge of the various backgrounds. The expected improvement varies between a factor of a few to an order of magnitude, but involves larger systematic uncertainties as the backgrounds 
are not precisely known. For this reason, the results we present use only this simple test described above.

\subsection{Models of Decaying Light Dark Matter}

In this section, we outline several simple scenarios that can accommodate LDM, and we place constraints on the model parameter space. The models below should be viewed as benchmarks that are not, however, complete. In particular, we do not discuss the production mechanism that results in the observed relic abundance. In the next section, we will derive "model-independent" constraints, where the results are presented as generic constraints on the lifetime versus mass for a given decay topology.

\subsubsection{Hidden Photino}

Consider a supersymmetric hidden sector, with an additional $U(1)_{d}$ gauge group [4, 7,70-73]. We assume that the SM and hidden sector can interact with each other through gauge kinetic mixing [74,75],

$$
-\frac{\epsilon}{2} \int d^{2} \theta \mathcal{W}_{d} \mathcal{W}_{Y}
$$

where $\mathcal{W}_{d}\left(\mathcal{W}_{Y}\right)$ are the supersymmetrized field strength of the hidden gauge group (hypercharge). The value of $\epsilon$ may naturally be of order $10^{-3}-10^{-4}$ when generated by integrating out heavy fields charged under both sectors. Conversely, if Eq. (6.7) results from higher dimensional operators, $\epsilon$ can be significantly smaller, as we will assume below in order to obtain MeV-GeV masses.

An interesting possibility is to have the hidden gaugino play the role of DM. To realize this, supersymmetry must be broken and communicated both to the visible and hidden sector. If the communication occurs through gauge mediation, the breaking 

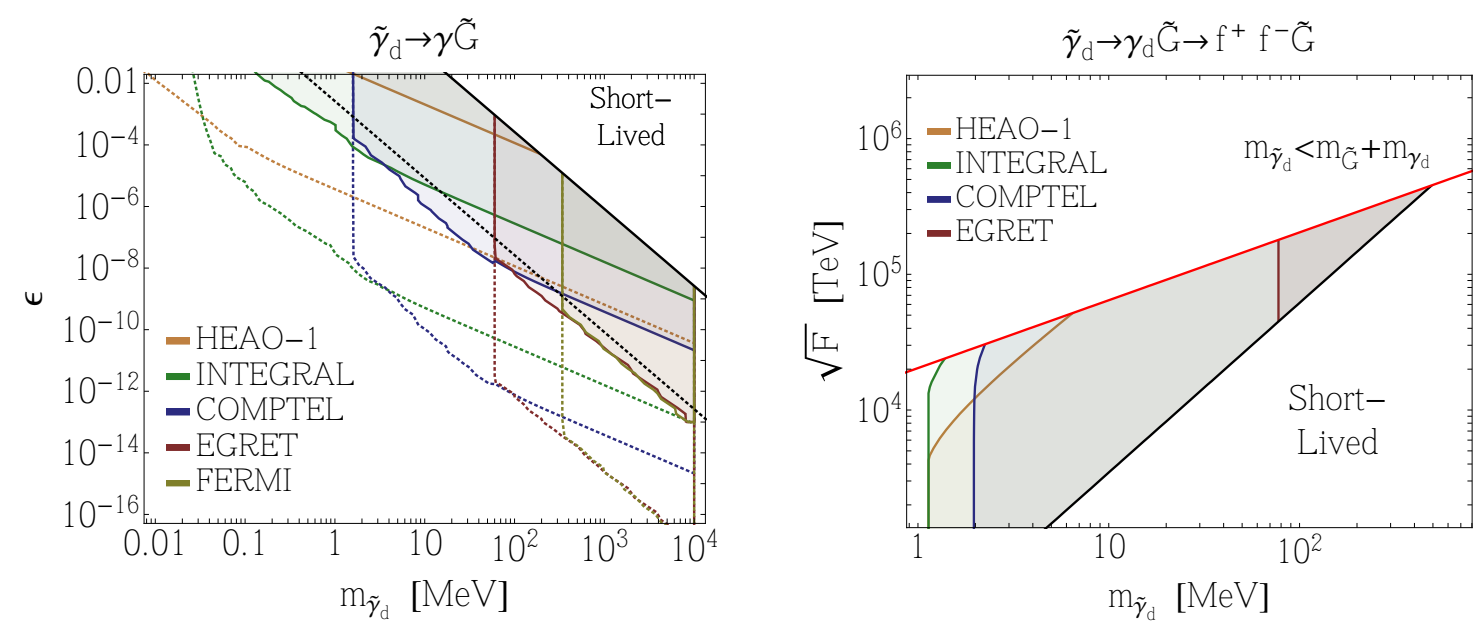

Figure 6.2: Constraints on hidden photino decay to left: gravitino and photon and right: gravitino and hidden photon (with the latter taken to have mass $m_{\gamma_{d}}=0.9 m_{\widetilde{\gamma}_{d}}$ and going to final state $f^{+} f^{-}$, with $f=e, \mu$ or $\pi$ ). In the left plot, the solid (dotted) lines are with $\sqrt{F}=10^{4}\left(10^{2}\right) \mathrm{TeV}$. The constraints are derived from the diffuse gamma- and X-ray data taken from HEAO-1 (orange), INTEGRAL (green), COMPTEL (blue), EGRET (red), and Fermi (yellow). In the "Short-Lived" region the DM lifetime is shorter than the age of Universe. Above the solid red line, the hidden photino is stable.

in the hidden sector may be significantly smaller than in the visible sector as supersymmetry breaking is transmitted to the hidden sector through D-term mixing [71]. As a consequence, the hidden photon mass is given by,

$$
m_{\gamma_{d}}^{2}=\epsilon g_{d}\left\langle D_{Y}\right\rangle \simeq(5 \mathrm{MeV})^{2}\left(\frac{\epsilon}{10^{-8}}\right)\left(\frac{g_{d}}{0.2}\right)\left(\frac{\sqrt{\left\langle D_{Y}\right\rangle}}{50 \mathrm{GeV}}\right)^{2}
$$

where $\left\langle D_{Y}\right\rangle=\left|\frac{g_{Y} v^{2} c_{2 \beta}}{4}\right|, v=246 \mathrm{GeV}$, and $\tan \beta=v_{u} / v_{d}$. In such a case, $\gamma_{d}$ and $\tilde{\gamma}_{d}$ are nearly degenerate, and $\tilde{\gamma}_{d}$ can decay to the gravitino and either a photon or a hidden photon, depending on whether the latter is heavier or lighter than $\tilde{\gamma}_{d}[7,72,73,76,77]$. The hidden photino lifetime is,

$$
\tau_{\tilde{\gamma}_{d} \rightarrow \gamma \tilde{G}} \simeq \epsilon^{-2}\left(\frac{m_{\tilde{\gamma}_{d}}^{5}}{16 \pi F^{2}}\right)^{-1} \simeq 3 \times 10^{23} \sec \left(\frac{10^{-8}}{\epsilon}\right)^{2}\left(\frac{10 \mathrm{MeV}}{m_{\tilde{\gamma}_{d}}}\right)^{5}\left(\frac{\sqrt{F}}{100 \mathrm{TeV}}\right)^{4}
$$



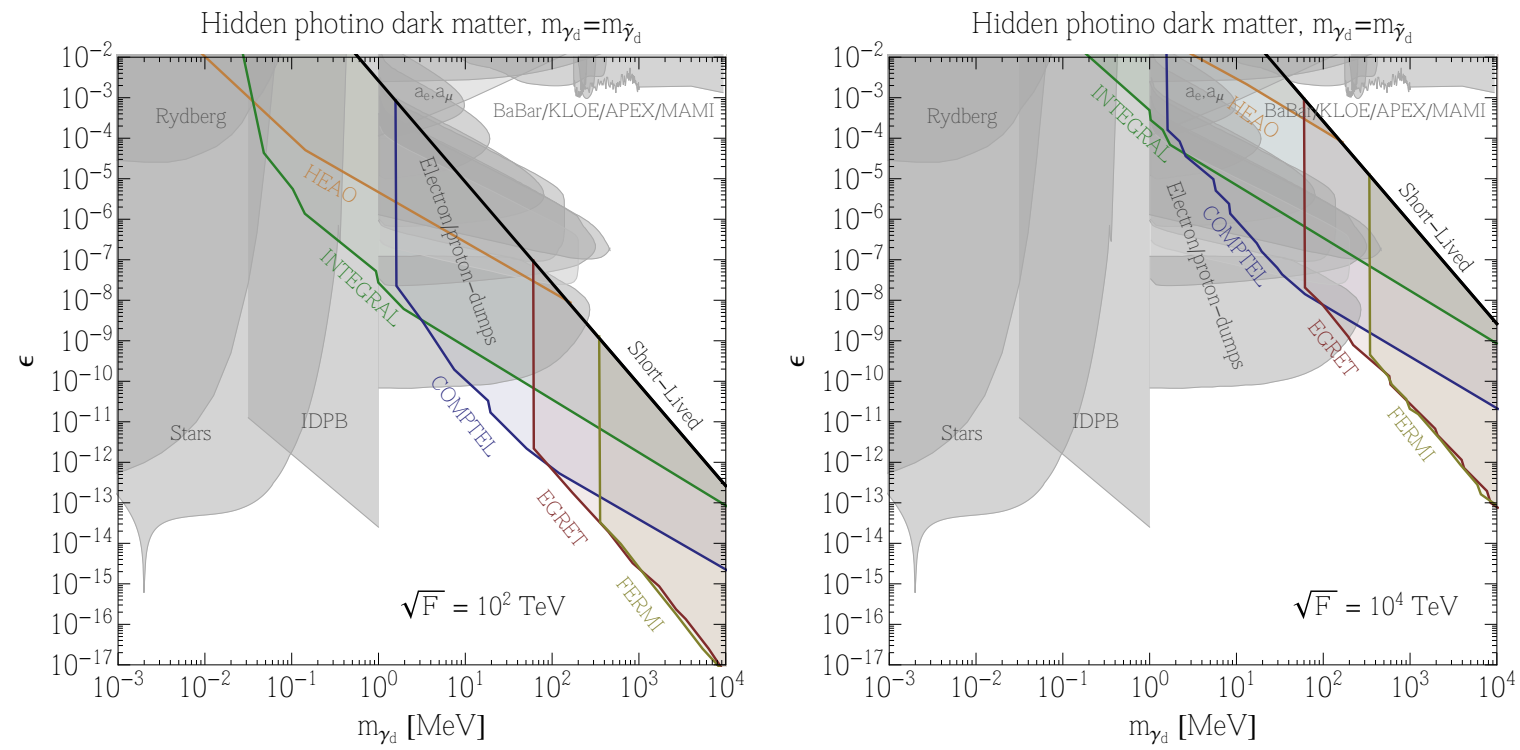

Figure 6.3: Constraints on a hidden photon in the hidden photino DM model for the case where the hidden photino decays to a photon and a gravitino, $\tilde{\gamma}_{d} \rightarrow \gamma \tilde{G}$, and with $\sqrt{F}=100 \mathrm{TeV}$ (left) or $\sqrt{F}=10^{4} \mathrm{TeV}$ (right). Gray shaded regions indicate constraints from beam-dump, fixed-target, and colliding beam experiments, stars, precision measurements, and from the intergalactic diffuse photon background (IDPB), while the colored regions show the gamma- or X-ray constraints as in Fig. 6.2. In the "Short-Lived" region the DM lifetime is shorter than the age of Universe. See text for more details.

for the decay to the photon and gravitino. This lifetime depends on several parameters, and can be much longer for lighter DM if the exact relation, Eq. (6.8), holds. Of course, $m_{\tilde{\gamma}_{d}}$ can be controlled by some other dynamics and hence be independent of $\epsilon$. Similarly for $\tilde{\gamma}_{d} \rightarrow \gamma_{d} \tilde{G}$ we have,

$$
\begin{aligned}
\tau_{\tilde{\gamma}_{d} \rightarrow \gamma_{d} \tilde{G}} & \simeq\left(\frac{m_{\tilde{\gamma}_{d}}^{5}}{16 \pi F^{2}}\right)^{-1}\left(1-\nu_{\gamma_{d}}^{2}\right)^{-4} \\
& =3 \times 10^{20} \sec \left(\frac{1 \mathrm{MeV}}{m_{\tilde{\gamma}_{d}}}\right)^{5}\left(\frac{\sqrt{F}}{10^{4} \mathrm{TeV}}\right)^{4}\left(1-\frac{m_{\gamma_{d}}^{2}}{m_{\mathrm{DM}}^{2}}\right)^{-4}
\end{aligned}
$$

Here, a long lifetime requires a slightly larger SUSY breaking scale. Note that the two possibilities lead to distinct indirect detection signals. In the first case one expects 
a spectral line, while in the second the spectrum is dominated by the FSR photons from the kinematically accessible charged particles that arise from the decay of the hidden photon.

The constraints for both cases are shown in Fig. 6.2. In the case of the line, we show the bounds in the $\epsilon-m_{\widetilde{\gamma}_{d}}$ plane, taking two choices for $\sqrt{F}$. For the case where the photino decays via a hidden photon, the constraints are presented on the $\sqrt{F}-m_{\widetilde{\gamma}_{d}}$ plane with the assumption $m_{\gamma_{d}}=0.9 m_{\widetilde{\gamma}_{d}}$. Above the solid red line, the hidden photino is stable. The photon spectrum for a variety of different decay channels may be derived from [78]. In both panels, the "Short-Lived" region indicates that the DM lifetime is shorter than the age of Universe.

Assuming $m_{\gamma_{d}} \simeq m_{\tilde{\gamma}_{d}}$, additional constraints exist from beam-dump [79,80], fixedtarget [81,82], and colliding-beam experiments [83]; precision measurements [84]; stars $[85,86]$; and from the intergalactic diffuse photon background (IDPB). This final constraint is valid for hidden photons below $2 m_{e} \simeq 1 \mathrm{MeV}$, as these can decay to three photons and contribute to the diffuse photon background [34,87]. For a summary of results see, e.g., [88]. These additional constraints are shown in Fig. 6.3 together with the limits derived here (and shown in the left panel of Fig. 6.2), for the case where the hidden photon decays directly to a photon and a gravitino, $\tilde{\gamma}_{d} \rightarrow \gamma \tilde{G}$. We note that some of these additional constraints are model dependent and may be evaded.

\subsubsection{Sterile Neutrino}

Under certain circumstances, a sterile neutrino, $\nu_{s}$, may act as DM (for reviews, see $[48,57])$. Due to its mixing with the active neutrinos, it may decay either via a 2- or 3-body channel. The leading diagrams that contribute to these decay channels are shown in Fig. 6.4. In its simplest form, the theory at low energy is described by two parameters: 

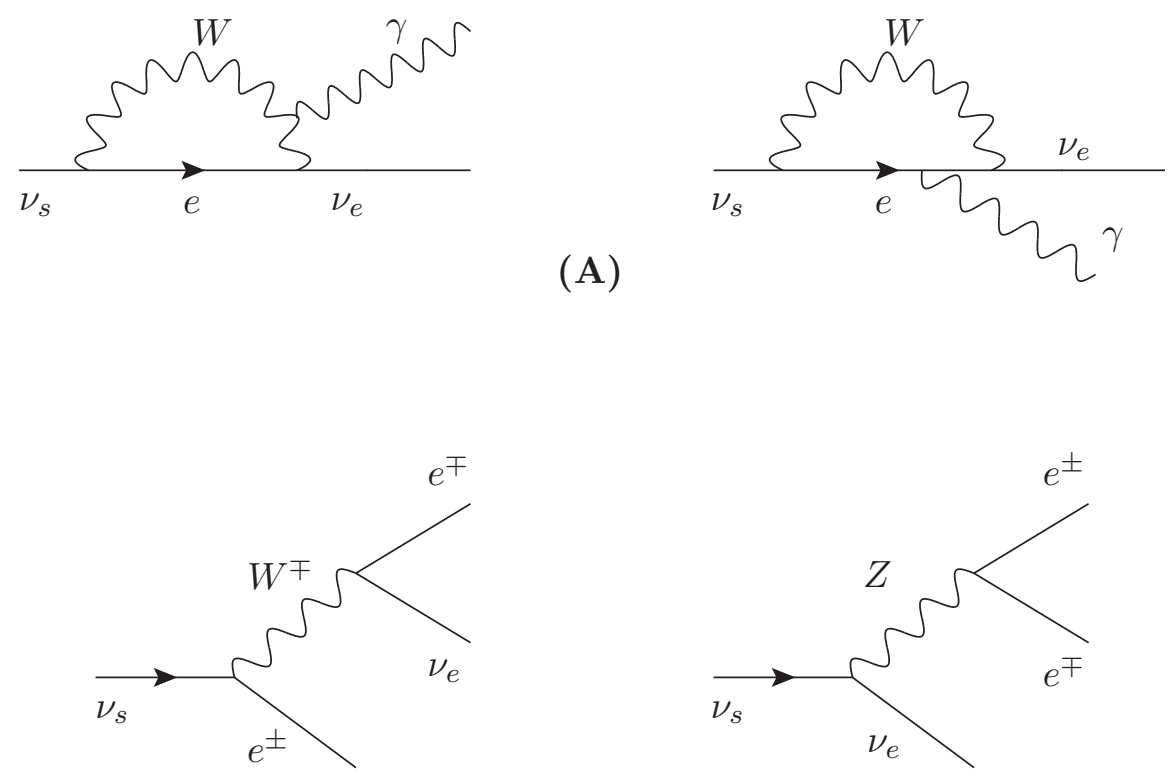

(B)

Figure 6.4: Decay channels for a sterile neutrino, $\nu_{s}$, through (A) a two-body radiative process $\left(\nu_{s} \rightarrow \nu_{\alpha} \gamma\right)$ and (B) charge- and neutral-current contributions to a three-body final state.

- $m_{s}$ - the sterile neutrino mass

- $\sin \theta_{\alpha}$ - the mixing angle between $\nu_{s}$ and active neutrinos of flavor $\alpha$; in what follows, we will only consider $\nu_{s}-\nu_{e}$ mixing.

The mixing above can be induced, for example, in supersymmetric theories with a superpotential, $W=X L L E^{c}$. The two-body decay rate for a Majorana neutrino is given by [89]

$$
\tau_{\nu_{s} \rightarrow \nu \gamma} \simeq\left(\frac{9 \alpha_{\mathrm{EM}} \sin ^{2} \theta}{1024 \pi^{4}} G_{F}^{2} m_{\chi}^{5}\right)^{-1} \simeq 1.8 \times 10^{17} \mathrm{~s}\left(\frac{10 \mathrm{MeV}}{m_{\chi}}\right)^{5}\left(\frac{\sin \theta}{10^{-8}}\right)^{-2}
$$

while the three-body decay rate is [90]

$$
\tau_{\nu_{s} \rightarrow \nu_{\alpha} e^{+} e^{-}} \simeq\left(\frac{c_{\alpha} \sin ^{2} \theta}{96 \pi^{3}} G_{F}^{2} m_{\chi}^{5}\right)^{-1} \simeq 2.4 \times 10^{15} \mathrm{~s}\left(\frac{10 \mathrm{MeV}}{m_{\chi}}\right)^{5}\left(\frac{\sin \theta}{10^{-8}}\right)^{-2} .
$$



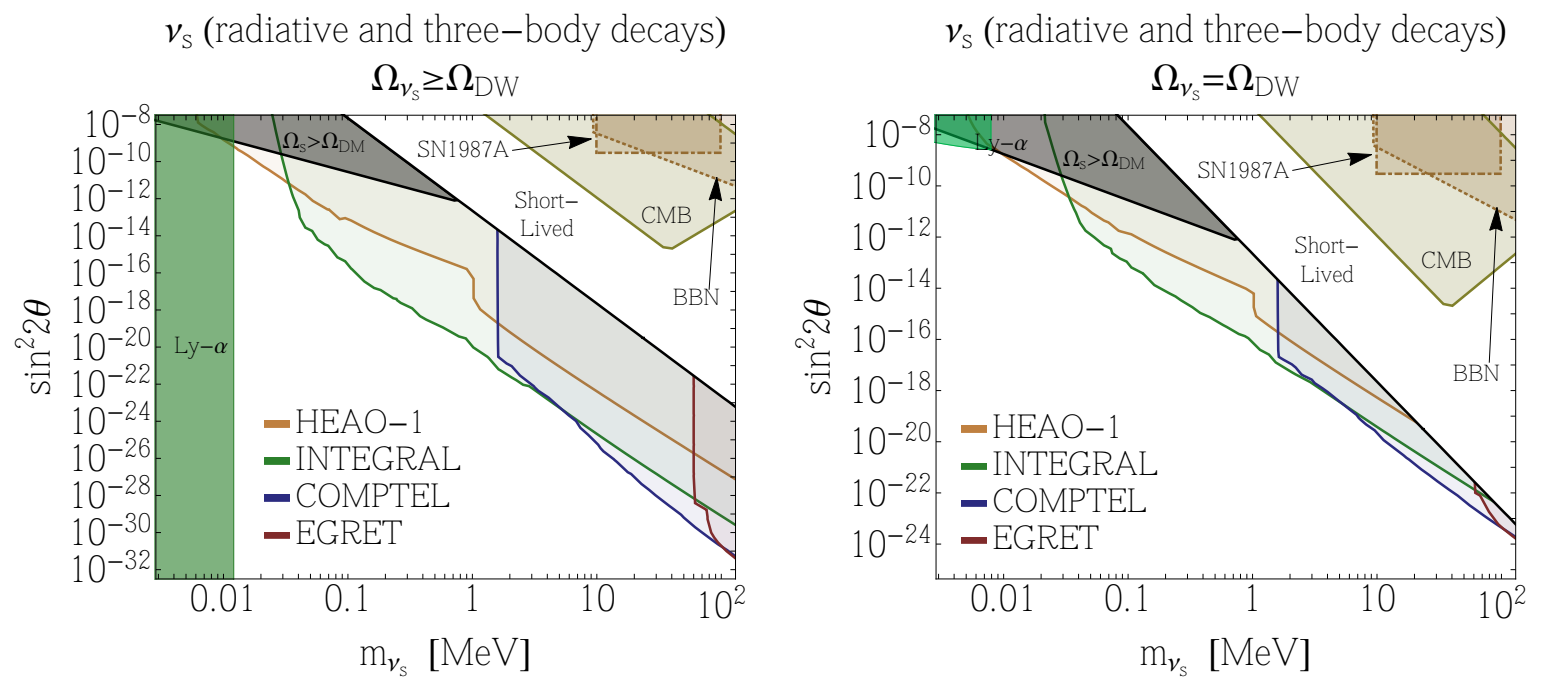

Figure 6.5: Constraints on the sum of sterile-neutrino decay to $\gamma \nu$ and $\nu e^{+} e^{-}$using the decay widths in Eqs. (6.11) and (6.12). The constraints from the diffuse gamma- and X-ray data are HEAO-1 (orange), INTEGRAL (green), COMPTEL (blue), and EGRET (red). Within the solid black region, the neutrino energy density must be greater than the observed DM density. Above (below) the black solid line, the neutrino lifetime is shorter (longer) than the age of the Universe. Within the green boundaries, the sterile neutrino is ruled out by Ly- $\alpha$ forest data $[48,49]$. Two cases for the sterile-neutrino energy density are assumed. In the left plot, the density is assumed to precisely equal the DM energy density everywhere below the dark and light gray regions. In the right plot, the density is determined by the (irreducible) DW mechanism.

Here the neutrino flavor $\alpha=e, c_{\alpha}=\frac{1+4 \sin ^{2} \theta_{W}+8 \sin ^{4} \theta_{W}}{4} \simeq 0.59$ [90], and we are only considering decays to $e^{+} e^{-}$pairs. The resulting gamma-ray fluxes from both channels contribute at roughly similar levels once the splitting function is introduced.

The relic abundance of sterile neutrinos is model dependent and varies according to the specific production mechanism and dynamics in the early Universe. An irreducible and UV-insensitive contribution to the abundance of sterile neutrinos arises from the so-called Dodelson-Widrow (DW) mechanism [91] in which the neutrinos are produced via oscillations. Thus, in the absence of new dynamics at low temperature, 
one finds [48]

$$
\Omega_{s} \gtrsim 0.25\left(\frac{\sin ^{2} 2 \theta}{4.3 \times 10^{-13}}\right)\left(\frac{m_{s}}{\mathrm{MeV}}\right)^{1.8} .
$$

Additional contributions may arise from, e.g., non-thermal production [8] or due to an extended Higgs sector $[92,93]$.

In order to place model-independent bounds on the parameter space of sterile neutrinos, we consider two different possibilities for the size of the sterile-neutrino relic abundance. First, we consider an unspecified UV mechanism that contributes to the DM density in those regions where the DM is under-abundant, setting $\Omega_{\nu_{s}}=\Omega_{\mathrm{DM}}$. Next, we assume the relic abundance is determined solely by the DW mechanism and, depending on the mixing angle and mass, $\Omega_{\nu_{s}}$ can be greater than or less than $\Omega_{\mathrm{DM}}$. We show our bounds for both these cases in the left and right panel of Fig. 6.5, respectively, in the $m_{\nu_{s}}-\sin ^{2} 2 \theta$ plane. In addition, we show existing bounds from the observation of the Lyman- $\alpha$ forest [49] and the overclosure region, in which the neutrino density produced by the DW mechanism exceeds the observed DM density. We also show the region where the sterile-neutrino lifetime is shorter than the age of the Universe, and hence it cannot act as DM. Several additional constraints exist on sterile neutrinos, for example, from the power spectrum of large scale structure [94] and of the CMB [94], from BBN [95], and from Supernova-1987A [96]. However, these constraints lie in the region where either the lifetime is too short or where the DM density is too high.

\subsubsection{Gravitino Dark Matter}

Another interesting possibility is gravitino DM [97-104]. The gravitino may be unstable on cosmological timescales and here we consider gravitino decays induced by R-parity violating (RPV) interactions [99-101]. Since we are interested in light DM, we will focus on the RPV operator that allows the gravitino to decay to leptons, $W=\lambda_{i j k} \ell_{i} \ell_{j} e_{k}^{c}$. A small coefficient $\lambda$ in the RPV vertex can ensure that the gravitino 


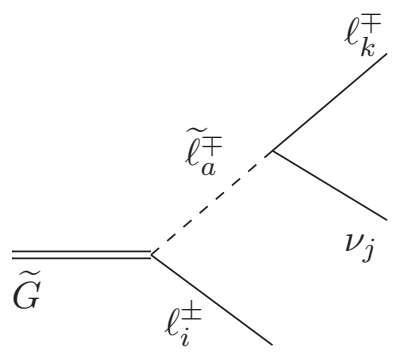

(A)

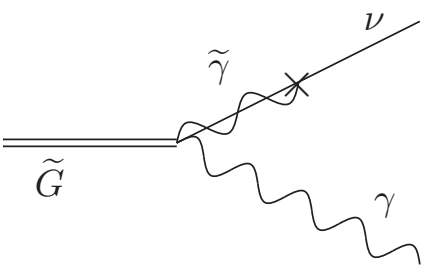

(B)

Figure 6.6: Feynman diagrams for $\widetilde{G}$ decay through (A) an off-shell slepton to a three-body final state $\left(\widetilde{G} \rightarrow \ell_{i}^{ \pm} \nu_{j} \ell_{k}^{\mp}\right)$ and (B) a two-body radiative process $\left(\widetilde{G} \rightarrow \nu_{\alpha} \gamma\right)$.

lifetime is longer than the age of the Universe.

Gravitinos are typically produced in three processes [97]: (i) gaugino scattering, dominantly at the re-heat temperature, (ii) freeze-out and decay of the lightest ordinary supersymmetric particle (LOSP, such as a neutralino), and (iii) freeze-in production from decays of visible sector particles, dominated at temperatures of order the superpartner masses.

Once gravitinos are produced with the observed relic abundance, their decay rate is controlled by the strength of the RPV vertex, as well as by the mass of the observable superpartners. The RPV operator considered here allows decays in one of two ways, as shown in the diagrams of Fig. 6.6. First, through an off-shell slepton, one has $\tilde{G} \rightarrow \nu_{j} \ell_{i}^{+} \ell_{k}^{-}$. This process is suppressed both by three-body phase space and by the slepton propagator, which gives an additional factor proportional to $\left(m_{3 / 2} / \tilde{m}\right)^{4}$, where $\tilde{m}$ is the slepton mass. One finds [101]

$$
\begin{aligned}
\tau_{\widetilde{G} \rightarrow \nu_{j} \ell_{i}^{+} \ell_{k}^{-}} & \simeq\left[\frac{\left|\lambda_{i j k}\right|^{2}}{3(32)^{2} \pi^{3}} \frac{m_{3 / 2}^{3}}{m_{\mathrm{Pl}}^{2}} F\left(\frac{\tilde{m}}{m_{3 / 2}}\right)\right]^{-1} \\
& \simeq 1.0 \times 10^{53} \mathrm{~s}\left(\frac{10^{-4}}{\lambda_{i j k}}\right)^{2}\left(\frac{10 \mathrm{MeV}}{m_{3 / 2}}\right)^{7}\left(\frac{\tilde{m}}{1 \mathrm{TeV}}\right)^{4}
\end{aligned}
$$



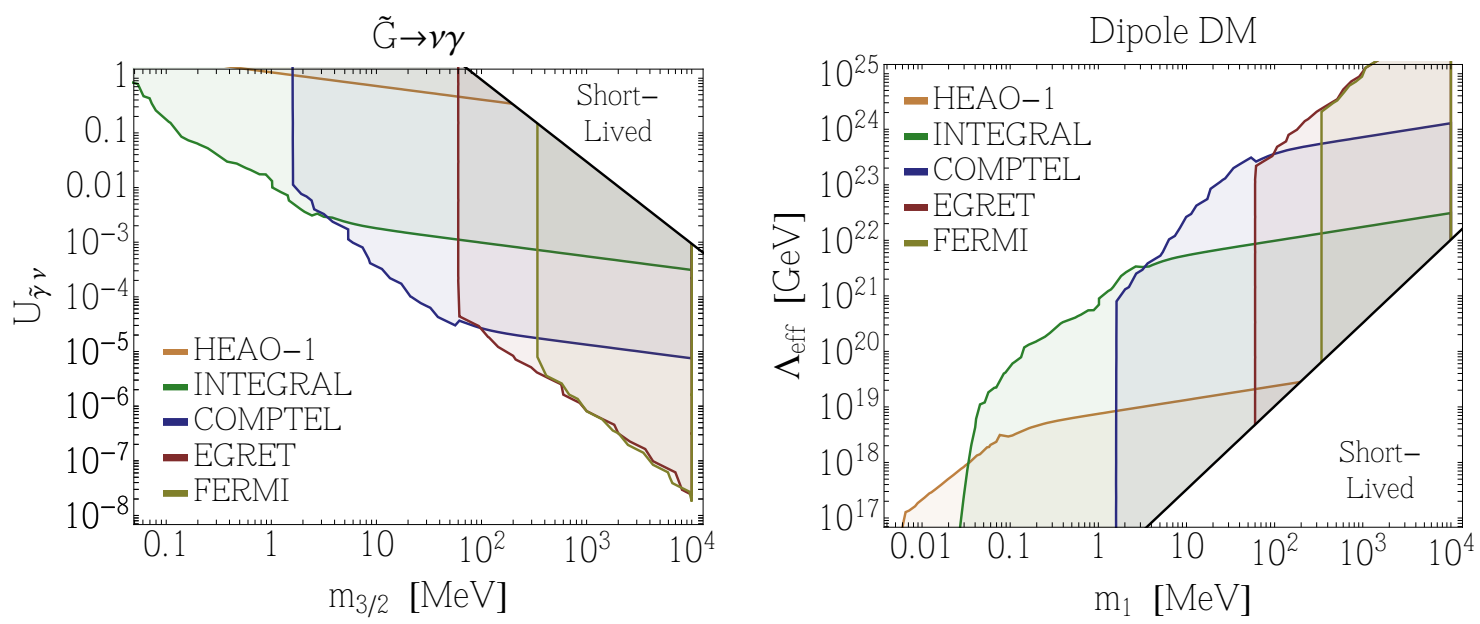

Figure 6.7: Left: Constraints on photino-neutrino mixing from RPV gravitino decay. Right: Constraints on the effective cutoff scale for DM with a dipole interaction. Regions as in Fig. 6.2.

where $m_{\mathrm{Pl}}=M_{\mathrm{Pl}} / \sqrt{8 \pi}=2.4 \times 10^{18} \mathrm{GeV}$ is the reduced Planck scale and $F(x) \simeq$ $1 /\left(30 x^{4}\right)$; a more exact expression can been found in [100].

A second, two-body, decay mode is $\tilde{G} \rightarrow \gamma \nu$, which usually dominates the decay width [101] and gives stronger bounds. It is induced by a mixing between the photino and the neutrino, $\left|U_{\tilde{\gamma} \nu}\right|$, which occurs if the RPV terms induce a VEV for the sneutrino $[99,101]$ or via a loop with a charged lepton and slepton. This gives a gravitino lifetime $[98,99]$,

$$
\tau_{\widetilde{G} \rightarrow \nu \gamma}=\left(\frac{1}{32 \pi}\left|U_{\widetilde{\gamma} \nu}\right|^{2} \frac{m_{3 / 2}^{3}}{m_{\mathrm{Pl}}^{2}}\right)^{-1} \simeq 3.8 \times 10^{28} \mathrm{~s}\left(\frac{10 \mathrm{MeV}}{m_{3 / 2}}\right)^{3}\left(\frac{10^{-4}}{U_{\widetilde{\gamma} \nu}}\right)^{2} .
$$

In the left panel of Fig. 6.7 we show the constraints on the photino-neutrino mixing angle as a function of the gravitino mass. In deriving the bound we require that the gravitino has the observed DM relic abundance. We do not show limits from BBN as those depend strongly on the dominant production mechanism and hence on the re-heat temperature and the spectrum of the superpartners [104]. 


\subsubsection{Dipole DM}

The dipole operator $\lambda \bar{\chi}_{2} \sigma^{\mu \nu} \chi_{1} F_{\mu \nu} / \Lambda$ (with $\left.\sigma^{\mu \nu}=i\left[\gamma^{\mu}, \gamma^{\nu}\right]\right)$ induces $\chi_{1} \rightarrow \chi_{2} \gamma$, where $\chi_{1,2}$ are Dirac fermions. The lifetime is

$$
\begin{aligned}
\tau_{\text {dipole }} & =\left[\frac{m_{1}^{3}}{2 \pi \Lambda_{\text {eff }}^{2}}\left(1-\frac{m_{2}^{2}}{m_{1}^{2}}\right)^{3}\right]^{-1} \\
& \simeq 4.1 \times 10^{20} \mathrm{~s}\left(\frac{10 \mathrm{MeV}}{m_{1}}\right)^{3}\left(\frac{\Lambda_{\text {eff }}}{10^{19} \mathrm{GeV}}\right)^{2}
\end{aligned}
$$

with $\Lambda_{\text {eff }}=\Lambda / \lambda$, the effective cutoff scale of the theory. The outgoing photon has an energy $E_{\gamma}=\left(m_{1}^{2}-m_{2}^{2}\right) / 2 m_{1}$. In the right panel of Fig. 6.7, we show the limits on $\Lambda_{\text {eff }}$ versus the $\chi_{1}$ mass, $m_{1}$. Since the effective operator that controls the decay is dimension 5 and not higher-dimensional, the limits are exceptionally strong, constraining the effective cutoff scale to be very high (or conversely, the corresponding

coupling to be small, $\lambda \ll 1$ ). An approximate symmetry in the UV may be required to protect these decays.

\subsubsection{Dark (Pseudo-) Scalars}

As a final model for light DM, we consider two-body decays of diphotons or charged particles. If DM is a pseudoscalar decaying to two photons, its lifetime is [105]

$$
\tau_{\pi_{d} \rightarrow \gamma \gamma} \simeq\left(\frac{\alpha_{\mathrm{EM}}^{2} m_{\pi_{d}}^{3}}{288 \pi^{3} f_{\pi_{d}}^{2}}\right)^{-1} \simeq 1.1 \times 10^{20} \mathrm{~s}\left(\frac{10 \mathrm{MeV}}{m_{\pi_{d}}}\right)^{3}\left(\frac{f_{\pi_{d}}}{10^{15} \mathrm{GeV}}\right)^{2}
$$

Here $f_{\pi_{d}}$ is the decay constant in the hidden sector, which we assume is Abelian. This decay produces a spectral line at an energy $m_{\pi_{d}} / 2$. We show the constraint in the left panel of Fig. 6.8, from which it is clear that the scale of $f_{\pi_{d}}$ needs to be very high.

If $\mathrm{DM}$ is a scalar that decays to charged particles that produce photons through 

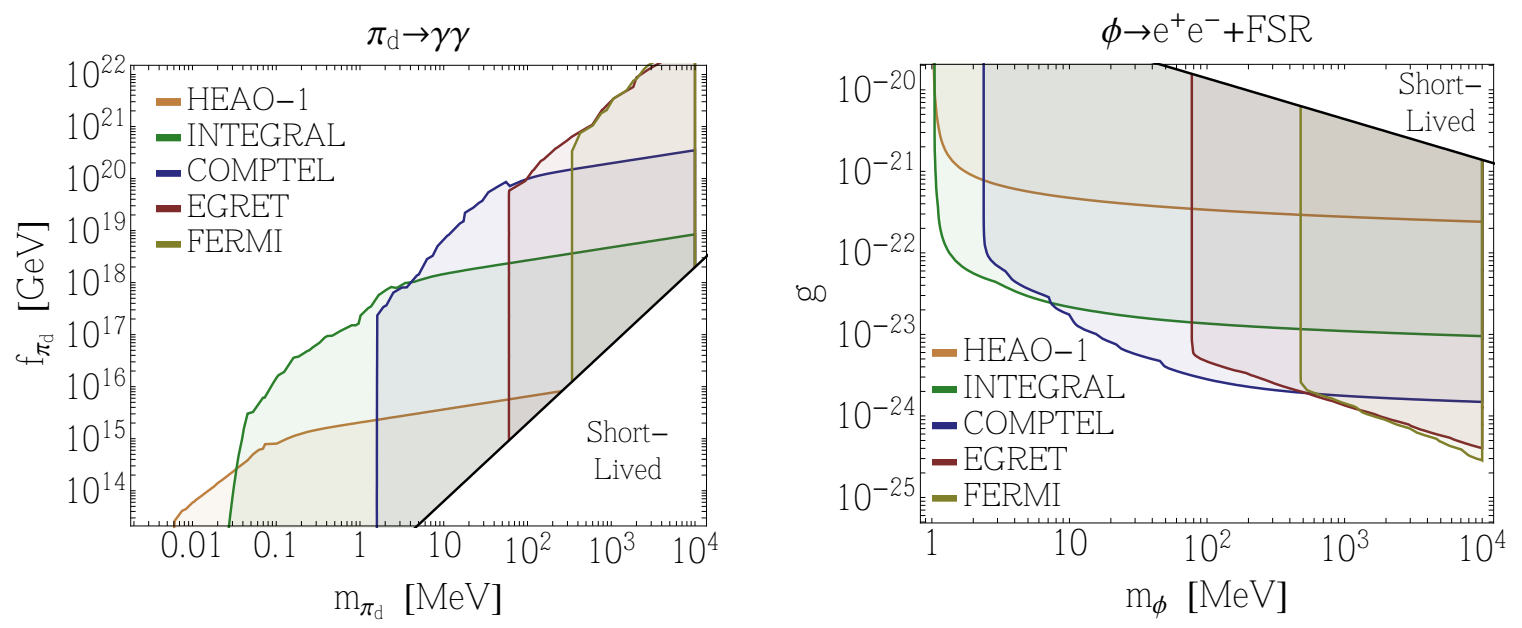

Figure 6.8: Constraints on the decay constant $f_{\pi_{d}}$ for a dark pseudoscalar decaying to diphotons (left) and the limits on the coupling of a hidden scalar in the case where it decays to $e^{+} e^{-}$(right). Regions as in Fig. 6.2.

FSR, e.g., $\phi \rightarrow e^{+} e^{-}$, the lifetime is

$$
\tau_{\phi \rightarrow e^{+} e^{-}}=\left[\frac{g^{2} m_{\pi_{d}}}{4 \pi}\left(1-4 \frac{m_{e}^{2}}{m_{\mathrm{DM}}^{2}}\right)^{3 / 2}\right]^{-1} \simeq 8.3 \times 10^{18} \mathrm{~s} \frac{10 \mathrm{MeV}}{m_{\phi}}\left(\frac{10^{-20}}{g_{a}}\right)^{2}
$$

The spectrum is bounded by the energies $0<E_{\gamma}<m_{\phi} / 2$. The constraints on the coupling $g$ are shown in the right panel of Fig. 6.8. As is apparent, tiny couplings are required for such DM to agree with observations.

\subsection{Model-Independent Bounds and Spectra}

In the previous section, we presented limits on specific model parameters. In this section, we fill in some of the details of the analysis there, and show bounds in terms of the lifetime only, making the constraints "model-independent." Despite the wide variety of possible decays that produce a photon signal, there are very few distinct event topologies of interest:

- Two- or three-body decays, with or without FSR. 
- Two-body cascade decays, where one or both of the decay products themselves subsequently decay to photons or charged particles.

In the limit of small outgoing particle masses, the differential decay width at low energies for each of these topologies may be written as a function of the total width, the photon energy, $E_{i}$, and the mass of the outgoing particle, $m_{i}$. We will use the small parameters

$$
\nu_{i}=\frac{m_{i}}{m_{\mathrm{DM}}}, \quad \lambda_{i}=\frac{E_{i}}{m_{\mathrm{DM}}},
$$

to expand our results.

When relevant in the model-independent bounds below, we only consider photons and electrons as SM final states. Typically these bounds will weaken moderately as new decay channels to additional charged or unstable heavier particles open up. One exception, however, is for the case where the decay products include $\pi^{0}$ s which consequently decay to photons. In such a case, a significant improvement in the limits is expected due to the sharp spectral feature.

\subsubsection{Two-Body Decays Involving a Photon}

We first consider two-body decays of DM directly to a photon and a neutral particle, or to two photons. Models that give line-like features include a hidden photino decaying to a gravitino and a photon via kinetic mixing, as discussed in Sec. 6.3.1. There are, of course, a profusion of other model-building possibilities that produce a monochromatic photon. These decays can produce one or two monochromatic photons with differential width,

$$
\frac{d N_{\text {two-body }}}{d E_{\gamma}}=\left\{\begin{array}{cl}
\delta\left(1-\nu_{2}^{2}-2 \lambda_{\gamma}\right) & (1 \text { photon }) \\
2 \delta\left(1-2 \lambda_{\gamma}\right) & (2 \text { photons })
\end{array} .\right.
$$




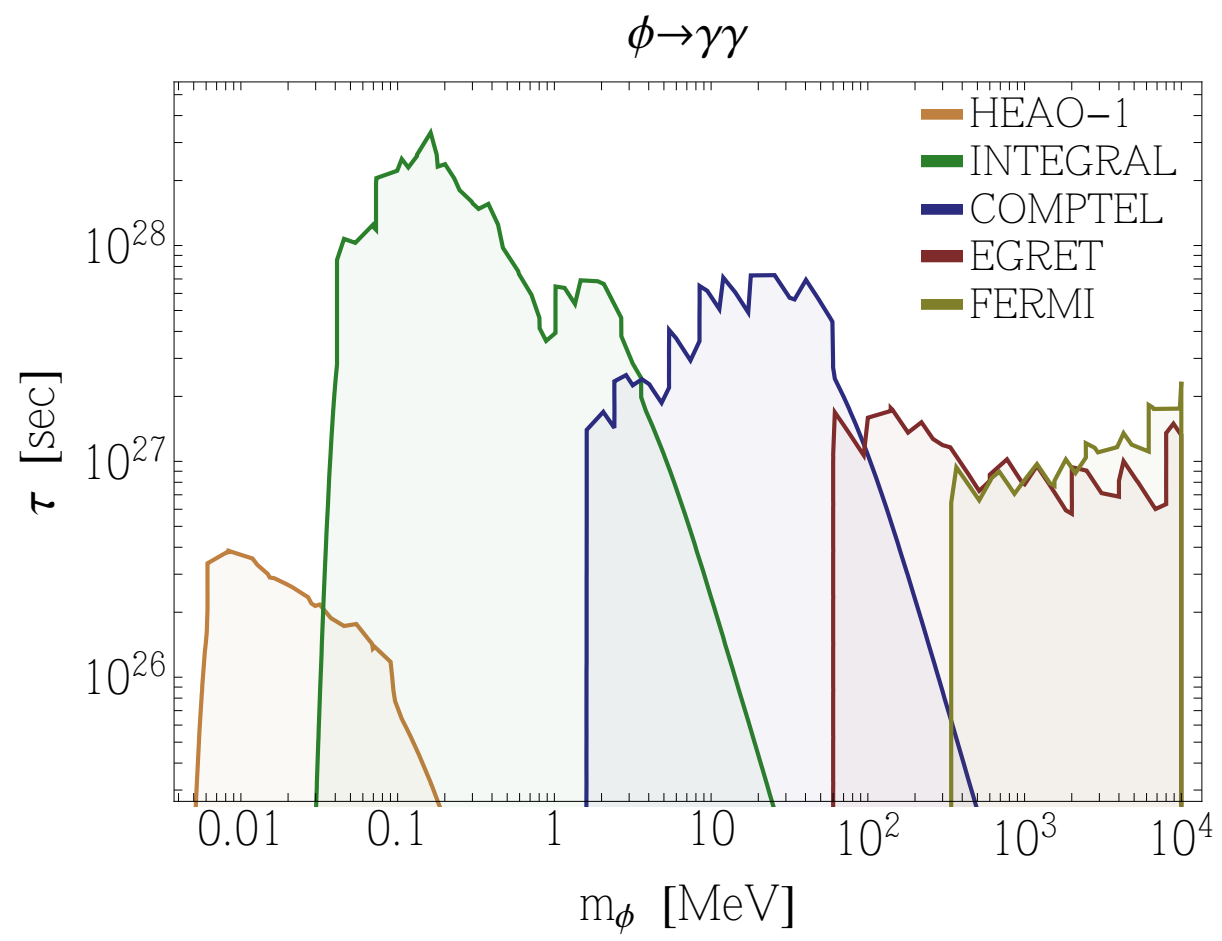

Figure 6.9: Bounds on the lifetime of a scalar DM, $\phi$, decaying to two photons. Regions as in Fig. 6.2.

Here $\nu_{2} \equiv m_{2} / m_{\text {DM }}$ refers to the mass of the outgoing decay partner, in the case of a single photon. The constraints on the lifetime for the decay to two photons are shown in Fig. 6.9.

\subsubsection{Two-Body Decays with FSR}

Two-body decays to charged particles produce photons through FSR. The differential width to photons is approximately given by integrating a $\delta$-function with the Altarelli-Parisi splitting function, as shown in Eq. (6.6), to give

$$
\frac{d N_{\phi \rightarrow e^{+} e^{-} \gamma}}{d E_{\gamma}} \simeq \frac{2 \alpha_{\mathrm{EM}}}{\pi E_{\gamma}}\left[1-2 \lambda_{\gamma}+\left(1-2 \lambda_{\gamma}+2 \lambda_{\gamma}^{2}\right) \ln \left(\frac{1-2 \lambda_{\gamma}}{\nu_{e}^{2}}\right)\right]
$$

where the spectrum is bounded by the energies $0<E_{\gamma}<m_{\phi} / 2$. We use the exact calculation of the three-body final state for the spectra and the exclusion regions in 

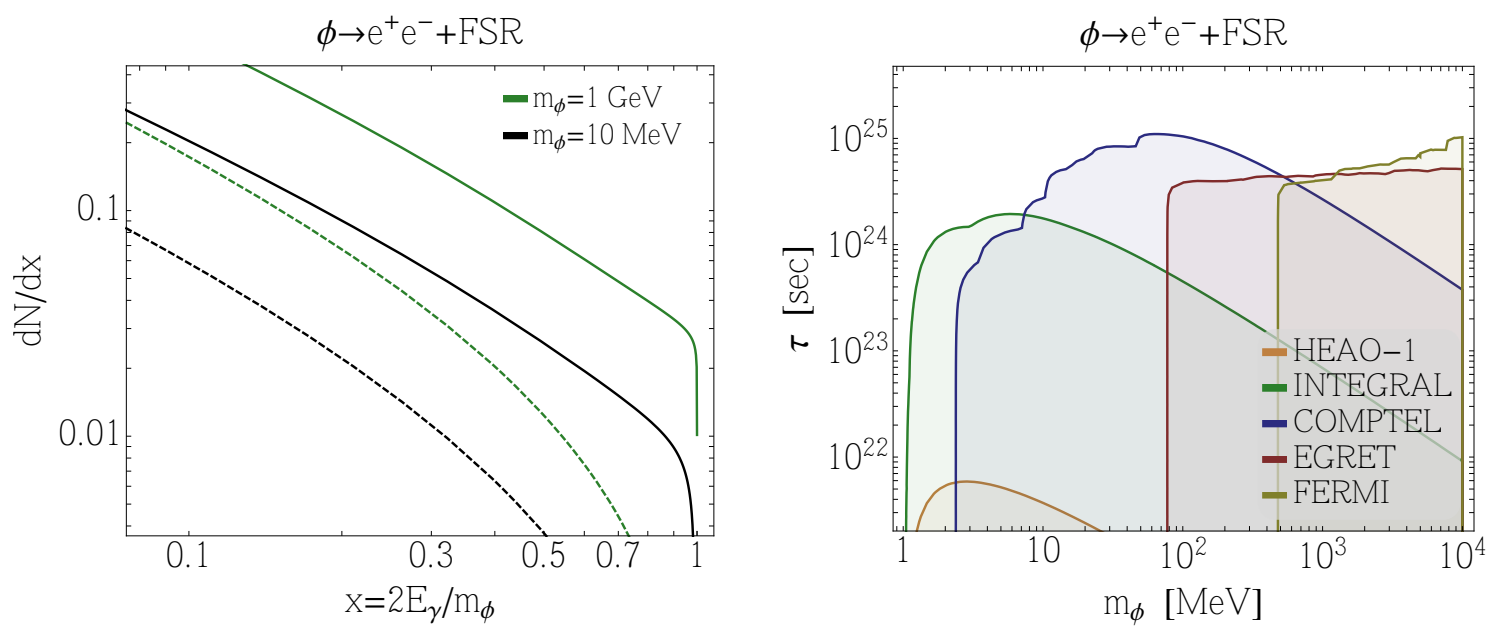

Figure 6.10: Left: Photon spectra from DM decay to $e^{+} e^{-}$, emitting final state radiation, as a function of $x=2 E_{\gamma} / m_{\mathrm{DM}}$. The spectrum of decays with galactic photons only is shown as the solid line, while the redshifted extragalactic spectrum is shown with dashed lines (see text for details). Right: Bounds on the DM decay lifetime for this process, with regions as in Fig. 6.2.

Fig. 6.10. In this figure, we show the dimensionless galactic photon spectrum

$$
\frac{d N}{d x}=\frac{m_{1}}{2} \frac{d N}{d E}
$$

as well as the redshifted extragalactic spectrum $d N_{\gamma, \mathrm{eg}} / d x$ (dashed lines). The extragalactic spectrum is calculated by performing the integral in Eq. (6.4)

$$
\frac{d N_{\gamma, \mathrm{eg}}}{d x}=\frac{\int_{0}^{\infty} d z \frac{d N}{d x(z)}\left[(1+z)^{3}+\kappa\right]^{-1 / 2}}{\int_{0}^{\infty} d z\left[(1+z)^{3}+\kappa\right]^{-1 / 2}},
$$

normalized such that the total number of photons for $0<x<1$ is equivalent for galactic and extragalactic photons.

As described above, this decay naturally arises if the DM is a light scalar. Furthermore, the decay to two leptons is a popular toy model that parameterizes possible DM decay and annihilation. The bounds for this case are shown on the right of Fig 6.10. As expected, they are a few orders of magnitude weaker than the bounds from the 

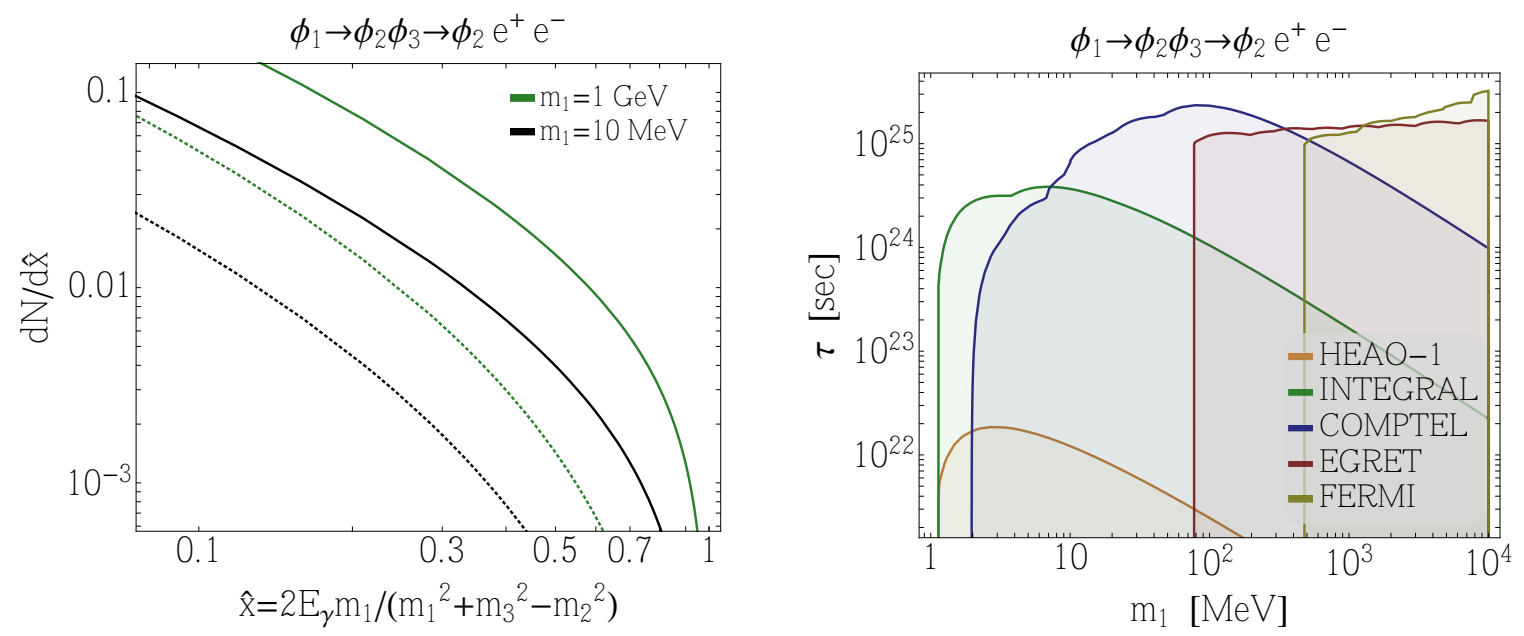

Figure 6.11: Left: Photon spectra versus $x=2 E_{\gamma} / m_{\mathrm{DM}}$ for DM decay to two neutral particles, where one of the neutral particles subsequently decays to $e^{+} e^{-}$, emitting final state radiation. The lines are as in Fig. 6.10. Right: Bounds on the DM decay lifetime for this process. Regions are as in Fig. 6.2.

monochromatic decay shown in the previous subsection.

\subsubsection{Two-Body Cascade Decays}

We next consider the case of DM decay to a pair of neutral particles, one of which subsequently decays to $e^{+} e^{-}: \phi_{1} \rightarrow \phi_{2} \phi_{3} \rightarrow \phi_{2} \ell^{+} \ell^{-}$. An example for a decay of this type was presented in Sec. 6.3.1 for the hidden photino model, where the hidden photino decays to a gravitino and hidden photon, which then subsequently decays to charged leptons: $\widetilde{\gamma}_{d} \rightarrow \widetilde{G} \gamma_{d} \rightarrow \widetilde{G} \ell^{+} \ell^{-}$. We derive the photon spectrum from these cascade decays from [78]. The spectrum for FSR resulting from a single boosted lepton is

$$
\begin{array}{r}
\frac{d N}{d E_{\gamma}}=\frac{2 \alpha_{\mathrm{EM}}}{\pi m_{1} \widehat{x}}\left\{\left[-1+\ln \left(\frac{m_{3}^{2}}{m_{\ell}^{2}}\right)\right]\left(2-\widehat{x}-\widehat{x}^{2}+2 \widehat{x} \ln \widehat{x}\right)+\left(\frac{\pi^{2}}{3}-1\right) \widehat{x}+\widehat{x}^{2}+\right. \\
\left.2 \widehat{x} \ln \widehat{x}+\left(2-\widehat{x}-\widehat{x}^{2}\right) \ln (1-\widehat{x})-2 \widehat{x} \operatorname{Li}_{2}(\widehat{x})\right\},
\end{array}
$$



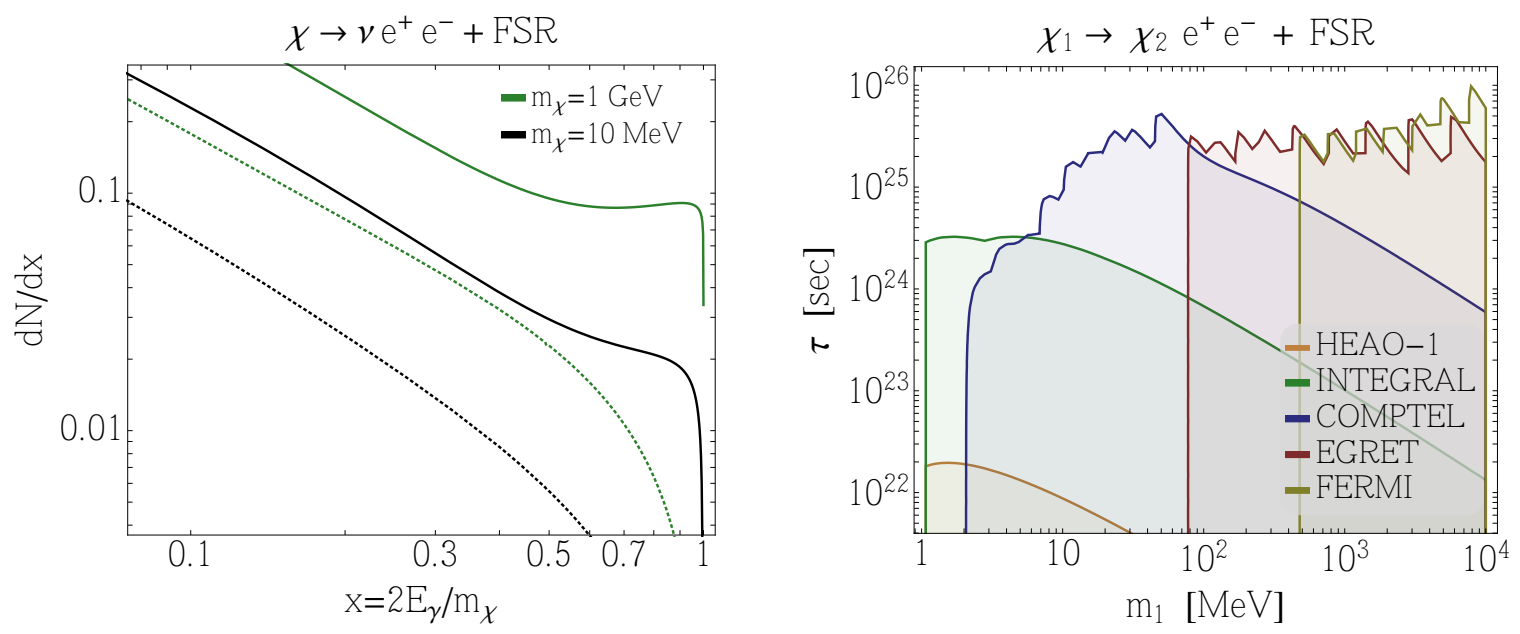

Figure 6.12: Left: Photon spectra versus $x=2 E_{\gamma} / m_{\mathrm{DM}}$ for DM decay to $e^{+} e^{-} \nu$, emitting final state radiation. The lines represent the galactic (solid) and extragalactic (dashed) spectra. Right: Bounds on the DM decay lifetime for this process. Regions as in Fig. 6.2.

where $\widehat{x}=2 m_{1} E_{\gamma} /\left(m_{1}^{2}+m_{3}^{2}-m_{2}^{2}\right)$. This spectrum, under the assumption of $m_{3}=$ $0.9 m_{1}$ and $m_{2}=0.01 m_{1}$, is shown on the left of Fig. 6.11 where the galactic (solid lines) and redshifted extragalactic (dashed lines) contributions are shown. As can be seen, Eq. (6.24) does not have a precise cutoff at $E_{\gamma}=m_{1} / 2$. However, as noted in [78], the number of unphysical photons produced with $E_{\gamma}>m_{1} / 2$ is second order in the expansion parameters and the effect of this error on the bounds is negligible.

The constraints on the lifetime of the decaying particle are shown on the right of Fig. 6.11, (with similar assumptions on $m_{2,3}$ as made in the left panel). These constraints are comparable to those on two-body + FSR models, and are considerably less constraining than those with monochromatic photons.

\subsubsection{Three-Body Decays with FSR}

Next we examine three-body DM decays, where the DM decays to a pair of charged particles plus a neutral particle. Our formula was specifically derived for the case of Weak decays of a sterile neutrino, $\nu_{s} \rightarrow \nu e^{+} e^{-}$(as we discussed in Sec. 6.3.2), though 
only minor changes result for a more generic decay $\phi_{1} \rightarrow \phi_{2} e^{+} e^{-}$.

The differential width of a fermionic DM decaying to $e^{+} e^{-} \nu$ via weak processes and including FSR is,

$\frac{d N_{D M, \mathrm{FSR}}}{d E_{\gamma}} \simeq \frac{2 \alpha_{\mathrm{EM}}}{\pi E_{\gamma}} \log \left(\frac{1-2 \lambda_{\gamma}}{\nu_{e}^{2}}\right)\left[1-\frac{11}{3} \lambda_{\gamma}+10 \lambda_{\gamma}^{2}+\frac{\lambda_{\gamma}\left(1+4 \sin ^{2} \theta_{W}\right)\left(1-6 \lambda_{\gamma}\right)}{12 c_{\alpha}}+\cdots\right]$

Here we neglect both the neutrino and the electron masses and "..." stands for higherorder terms in $\nu_{e}$. For the case of a decay process mediated by a heavy neutral scalar particle, the above remains the same with the omission of the last term.

The spectrum for the above is plotted on the left of Fig. 6.12 where, as before, the galactic (solid lines) and redshifted extragalactic (dashed lines) contributions are shown. The constraints on the lifetime are shown on the right of Fig. 6.12. We find the bounds to be similar in magnitude to the two-body + FSR case, however sensitivity to the endpoint feature in the spectrum is apparent and results in the wiggles displayed in the figure.

\subsubsection{Three-Body Decays Involving Photons}

Three body decays such as $\phi_{1} \rightarrow \phi_{2} \gamma \gamma$ are also possible. We remain agnostic about the UV completion and do not embed this interaction in any of the theories above. Nonetheless, we include it here for completeness.

To obtain bounds, we assume that this decay is induced by the higher-dimensional operator $\mathcal{O}=\frac{\beta}{4 \Lambda^{2}} \phi_{1} \phi_{2} F_{\mu \nu} F^{\mu \nu}$. We have,

$$
\frac{d N_{\phi_{1} \rightarrow \phi_{2} \gamma \gamma}}{d E_{\gamma}}=\frac{128 E_{\gamma}^{3}}{m_{1}^{4}} \frac{\left(1-\frac{\nu_{2}^{2}}{1-2 \lambda_{\gamma}}\right)^{3}}{1+28 \nu^{2}\left(1-\nu_{2}^{4}\right)-\nu_{2}^{8}+12 \nu_{2}^{2}\left(1+3 \nu_{2}^{2}+\nu_{2}^{4}\right) \ln \nu_{2}^{2}} .
$$

We see here that the width is exponentially sensitive to the energy in the limit $\nu_{2} \rightarrow 0$, which means that the photons from this decay are preferentially grouped near the 

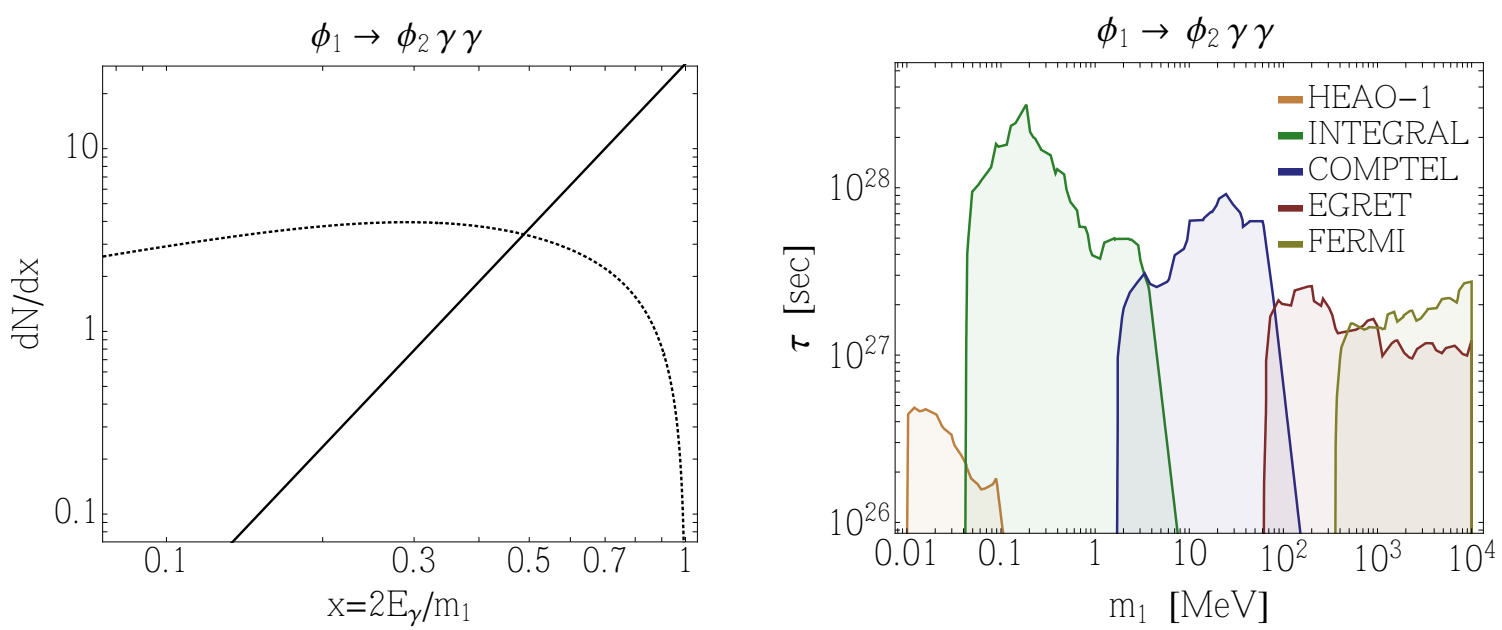

Figure 6.13: Left: Photon spectra versus $x=2 E_{\gamma} / m_{\mathrm{DM}}$ for DM three-body decay $\phi_{1} \rightarrow \phi_{2} \gamma \gamma$. The lines represent the galactic (solid) and redshifted extragalactic (dashed) spectra. Right: Bounds on the DM decay lifetime for this process, with regions as explained in Fig. 6.2.

DM mass. Consequently, for a given $m_{\chi}$, the constraint arises from a single bin in a given experiment.

We display the spectrum and constraint on the lifetime in Fig. 6.13, with the assumption $m_{2}=0$. In this limit, the differential spectrum is the same regardless of $m_{1}$. As expected, these bounds compare favorably to the monochromatic photon lines.

\subsection{Annihilating Light Dark Matter}

Here we consider bounds on annihilating DM, specializing to the case of annihilation to $e^{+} e^{-}$(see also [106]). The differential photon spectrum for this case is

$$
\begin{aligned}
& \frac{d N}{d E_{\gamma}}=\frac{2 \alpha_{\mathrm{EM}}}{\pi E_{\gamma}} \frac{1}{\left(1-\nu_{e}^{2}\right)^{3 / 2}}\left\{\delta\left(1-\nu_{e}^{2}\right)+\right. \\
& \left.\quad\left[1-\lambda_{\gamma}+\frac{1}{2} \lambda_{\gamma}^{2}-\nu_{e}^{2}\left(\frac{3}{2}-\lambda_{\gamma}\right)+\frac{1}{2} \nu_{e}^{4}\right] \ln \left(\frac{1-\lambda_{\gamma}-\delta}{1-\lambda_{\gamma}+\delta}\right)\right\},
\end{aligned}
$$




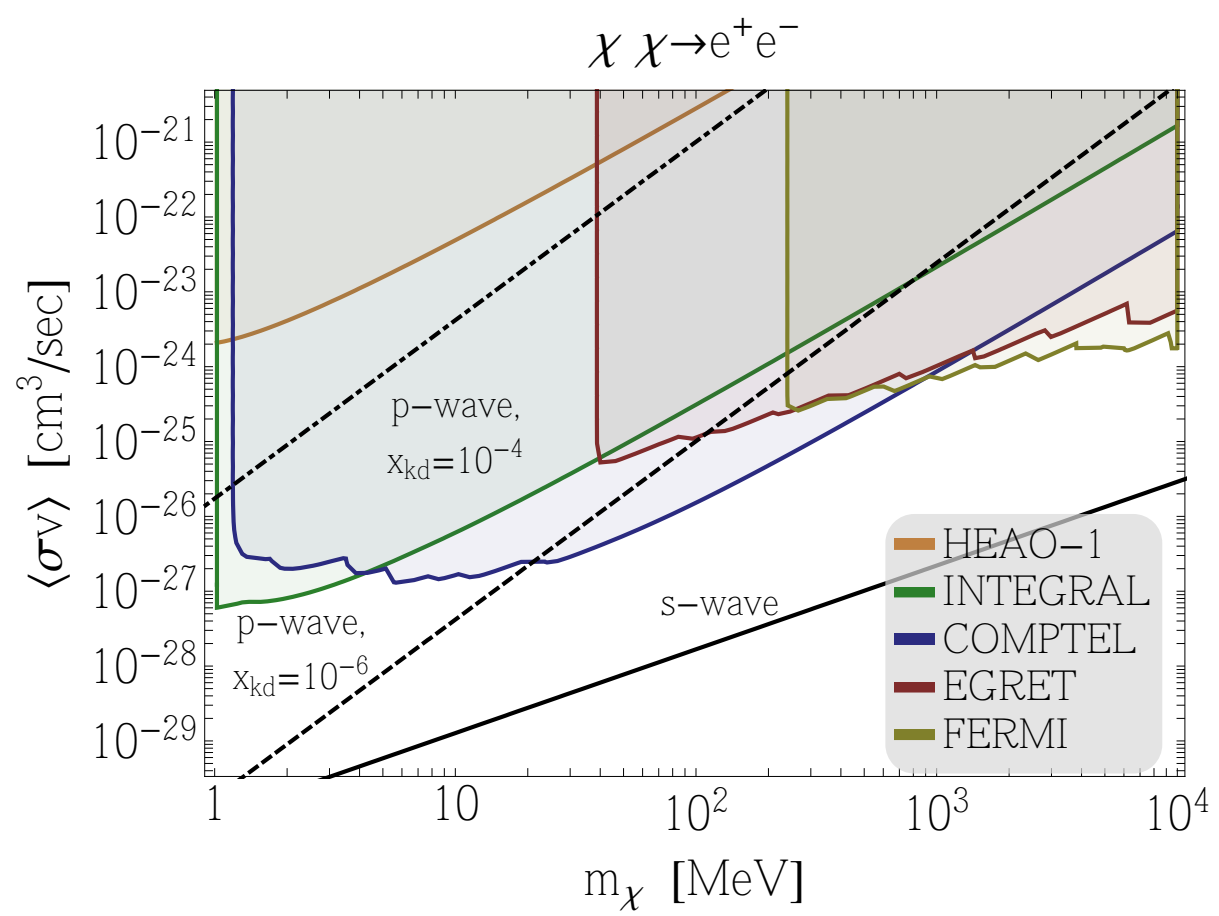

Figure 6.14: Bounds on the DM velocity-averaged annihilation cross-section $\langle\sigma v\rangle$ due to FSR off the process $\chi \chi \rightarrow e^{+} e^{-}$. Regions as in Fig. 6.2. Also shown is a comparison with the CMB constraint for DM annihilation that is $s$-wave (solid) or $p$-wave, the latter for two different kinetic-decoupling temperatures, $x_{\mathrm{kd}} \equiv T_{\gamma} / m_{\mathrm{DM}}=10^{-4}$ (dash-dot) and $10^{-6}$ (dashed line), where we take $T_{\gamma}=0.235 \mathrm{eV}$ at the $\mathrm{CMB}$ epoch (corresponding to $\left.z_{\mathrm{CMB}}=1000\right)$.

where we have defined $\delta=\sqrt{\left(1-\lambda_{\gamma}\right)\left(1-\lambda_{\gamma}-\nu_{e}^{2}\right)}$. The bounds are shown in Fig. 6.14. From Tab. 6.1, we see that these results are sensitive (within factors of a few) to the DM density profile (we use the NFW profile for all results), especially for experiments that observe regions near the center of the galaxy such as INTEGRAL and COMPTEL. For DM masses below $\sim 100 \mathrm{MeV}$ the bounds are stronger than the thermal annihilation cross-section around $3 \times 10^{-26} \mathrm{~cm}^{3} / \mathrm{s}$.

These bounds can be compared with those from CMB observations, which are very strong for $s$-wave processes. Indeed, for DM masses below $\sim 7 \mathrm{GeV}$, the annihilation cross-section must be smaller than the thermal annihilation cross-section of $3 \times 10^{-26} \mathrm{~cm}^{3} / \mathrm{s}$. At first sight, it appears that the diffuse photon bounds are 
not competitive with the CMB bounds. However, $p$-wave annihilation rates may be larger in the galaxy today relative to the $\mathrm{CMB}$ epoch if the velocity of the $\mathrm{DM}$ at recombination is smaller than the galactic velocity which we take to be, $v_{0}=220$ $\mathrm{km} / \mathrm{sec}$.

The velocity of the DM at recombination depends on the kinetic decoupling temperature. As long as the DM remains kinetically coupled to the plasma, its velocity is $v_{\mathrm{DM}} \sim \sqrt{3 T_{\gamma} / m_{\mathrm{DM}}}$. Once the DM kinetically decouples, however, it cools much more quickly: its temperature at redshift $z$ is $T_{\mathrm{DM}}=T_{\mathrm{kd}}\left(\frac{z}{z_{\mathrm{kd}}}\right)^{2}$, for a kinetic decoupling temperature $T_{\mathrm{kd}}$ at redshift $z_{\mathrm{kd}}$. As a result, the DM velocity is

$$
\begin{aligned}
v_{\mathrm{DM}} & =\sqrt{3 T_{\mathrm{DM}} / m_{\mathrm{DM}}}=\sqrt{3} x_{\gamma} x_{\mathrm{kd}}^{-1 / 2} \\
& \simeq 2 \times 10^{-4}\left(\frac{T_{\gamma}}{1 \mathrm{eV}}\right)\left(\frac{1 \mathrm{MeV}}{m_{\mathrm{DM}}}\right)\left(\frac{10^{-4}}{x_{\mathrm{kd}}}\right)^{1 / 2}
\end{aligned}
$$

where we define $x_{i} \equiv T_{i} / m_{\mathrm{DM}}$. The above is easily smaller than the observed galactic velocity, even for very light DM.

We show in Fig. 6.14 the CMB constraint from $s$-wave processes, as well as the constraint from $p$-wave processes for $x_{\mathrm{kd}}=10^{-4}$ and $10^{-6}$, taking $T_{\gamma}=0.235 \mathrm{eV}$ at the $\mathrm{CMB}$ epoch (corresponding to $z_{\mathrm{CMB}}=1000$ ). In order to compare the galactic and CMB constraints for both $s$ - and $p$-wave annihilation, we show contours of $\langle\sigma v\rangle \propto\left(v_{\mathrm{DM}} / v_{0}\right)^{2(n-1)}$, where $n=1(2)$ for $s(p)$-wave. We can see that the CMB constraints are always stronger than the diffuse photon constraints for $s$-wave annihilation. However, the diffuse constraints are stronger than the CMB constraints for $p$-wave annihilation, especially for larger kinetic-decoupling temperatures where the DM is colder. 


\subsection{Conclusions and Future Improvements}

In this paper, we considered simplified models of DM with masses $\mathcal{O}$ (few $\mathrm{KeV}) \lesssim$ $m_{\mathrm{DM}} \lesssim \mathcal{O}($ few $\mathrm{GeV})$, that can give rise to observable signals in X-ray and gammaray observatories via decays or annihilations. We found that bounds from HEAO-1, COMPTEL, INTEGRAL/SPI, EGRET and Fermi, even without dedicated searches, can already be very strong, even under conservative assumptions.

For decaying light $\mathrm{DM}$, constraints on the lifetime, $\tau_{\mathrm{DM}}$, are in the range $10^{24}-$ $10^{28} \mathrm{~s}$, where the weaker bounds typically apply in the case where DM decays to photons via FSR, while the stricter bounds apply when DM decays directly to photons. On the other hand, for DM that annihilates to two electrically charged SM particles, we find that below a few hundred $\mathrm{MeV}$ the annihilation cross-section must be lower than the canonical thermal relic $s$-wave annihilation cross-section. In this case, the existing CMB bounds are found to be stronger. However, for $p$-wave suppressed annihilation, the CMB bounds become weaker than the diffuse constraints as the kinetic-decoupling temperature increases (and the DM at CMB becomes colder).

In addition to model-independent constraints, we also placed limits on specific benchmark models of light DM: hidden-photino DM, sterile-neutrino DM, gravitino DM, dipole DM and hidden (pseudo-) scalar DM. We found that the constraints from decaying DM are often stronger than other existing experimental, astrophysical, or cosmological constraints.

We conclude that X-ray and gamma-ray observatories provide a powerful and independent probe of light DM. To improve on the results presented here, dedicated searches are needed, where better background studies and optimized regions in the sky are considered. With the results above, the authors strongly encourage new studies in the hope of significantly widening the search window for dark matter. 


\section{Acknowledgements}

We thank Kfir Blum, Germán Arturo Gomez-Vargas, Shmuel Nussinov, Javier Redondo, Robert Shrock, and Andrew Strong for helpful discussions. RE is supported in part by the DoE Early Career research program DESC0008061 and by a Sloan Foundation Research Fellowship. SDM and KZ are supported by the DoE under contract de-sc0007859, by NSF CAREER award PHY 1049896, and by NASA Astro-

physics Theory grant NNX11AI17G. SDM is supported by the Fermilab Fellowship in Theoretical Physics. Fermilab is operated by Fermi Research Alliance, LLC, under Contract No. DE-AC02-07CH11359 with the United States Department of Energy. TV is supported in part by a grant from the Israel Science Foundation, the US-Israel Binational Science Foundation, the EU-FP7 Marie Curie, CIG fellowship and by the I-CORE Program of the Planning and Budgeting Committee and The Israel Science Foundation (grant NO 1937/12). 


\section{Bibliography}

[1] C. Boehm, D. Hooper, J. Silk, M. Casse and J. Paul, Phys. Rev. Lett. 92 (2004) 101301 [astro-ph/0309686].

[2] C. Boehm, P. Fayet and J. Silk, Phys. Rev. D 69 (2004) 101302 [hep$\mathrm{ph} / 0311143]$.

[3] M. Pospelov, A. Ritz and M. B. Voloshin, Phys. Lett. B 662, 53 (2008) [arXiv:0711.4866 [hep-ph]].

[4] D. Hooper and K. M. Zurek, Phys. Rev. D 77, 087302 (2008) [arXiv:0801.3686 [hep-ph]].

[5] J. L. Feng and J. Kumar, Phys. Rev. Lett. 101, 231301 (2008) [arXiv:0803.4196 [hep-ph]].

[6] D. E. Kaplan, M. A. Luty and K. M. Zurek, Phys. Rev. D 79, 115016 (2009) [arXiv:0901.4117 [hep-ph]].

[7] R. Essig, J. Kaplan, P. Schuster and N. Toro, [arXiv:1004.0691 [hep-ph]].

[8] A. Falkowski, J. T. Ruderman and T. Volansky, JHEP 1105, 106 (2011) [arXiv:1101.4936 [hep-ph]].

[9] R. Essig, J. Mardon and T. Volansky, Phys. Rev. D 85, 076007 (2012) [arXiv:1108.5383 [hep-ph]].

[10] N. Borodatchenkova, D. Choudhury and M. Drees, Phys. Rev. Lett. 96 (2006) 141802 [hep-ph/0510147].

[11] F. J. Petriello, S. Quackenbush and K. M. Zurek, Phys. Rev. D 77, 115020 (2008) [arXiv:0803.4005 [hep-ph]]. 
[12] Y. Gershtein, F. Petriello, S. Quackenbush and K. M. Zurek, Phys. Rev. D 78, 095002 (2008) [arXiv:0809.2849 [hep-ph]].

[13] J. Goodman, M. Ibe, A. Rajaraman, W. Shepherd, T. M. P. Tait and H. -B. Yu, Phys. Rev. D 82, 116010 (2010) [arXiv:1008.1783 [hep-ph]].

[14] P. J. Fox, R. Harnik, J. Kopp and Y. Tsai, Phys. Rev. D 84, 014028 (2011) [arXiv:1103.0240 [hep-ph]].

[15] R. Essig, J. Mardon, M. Papucci, T. Volansky, Y. Zhong, to appear.

[16] R. Essig, A. Manalaysay, J. Mardon, P. Sorensen and T. Volansky, Phys. Rev. Lett. 109 (2012) 021301 [arXiv:1206.2644 [astro-ph.CO]].

[17] P. W. Graham, D. E. Kaplan, S. Rajendran and M. T. Walters, Phys. Dark Univ. 1 (2012) 32 [arXiv:1203.2531 [hep-ph]].

[18] B. Batell, M. Pospelov and A. Ritz, Phys. Rev. D 80 (2009) 095024 [arXiv:0906.5614 [hep-ph]].

[19] P. deNiverville, M. Pospelov and A. Ritz, Phys. Rev. D 84 (2011) 075020 [arXiv:1107.4580 [hep-ph]].

[20] P. deNiverville, D. McKeen and A. Ritz, Phys. Rev. D 86 (2012) 035022 [arXiv:1205.3499 [hep-ph]].

[21] R. Dharmapalan et al. [MiniBooNE Collaboration], arXiv:1211.2258 [hep-ex].

[22] E. Izaguirre, G. Krnjaic, P. Schuster and N. Toro, arXiv:1307.6554 [hep-ph].

[23] M. D. Diamond and P. Schuster, arXiv:1307.6861 [hep-ph].

[24] S. Galli, F. Iocco, G. Bertone and A. Melchiorri, Phys. Rev. D 80, 023505 (2009) [arXiv:0905.0003 [astro-ph.CO]]. 
[25] T. R. Slatyer, N. Padmanabhan and D. P. Finkbeiner, Phys. Rev. D 80, 043526 (2009) [arXiv:0906.1197 [astro-ph.CO]].

[26] D. P. Finkbeiner, S. Galli, T. Lin and T. R. Slatyer, Phys. Rev. D 85, 043522 (2012) [arXiv:1109.6322 [astro-ph.CO]].

[27] S. Galli, F. Iocco, G. Bertone and A. Melchiorri, Phys. Rev. D 84, 027302 (2011) [arXiv:1106.1528 [astro-ph.CO]].

[28] M. Ackermann et al. [LAT Collaboration], Phys. Rev. D 86 (2012) 022002 [arXiv:1205.2739 [astro-ph.HE]].

[29] M. Ackermann et al. [LAT Collaboration], Astrophys. J. 761, 91 (2012) [arXiv:1205.6474 [astro-ph.CO]].

[30] G. A. Gomez-Vargas, M. A. Sanchez-Conde, J. -H. Huh, M. Peiro, F. Prada, A. Morselli, A. Klypin and D. G. Cerdeno et al., arXiv:1308.3515 [astro-ph.HE].

[31] M. Papucci and A. Strumia, JCAP 1003 (2010) 014 [arXiv:0912.0742 [hep-ph]].

[32] M. Cirelli, P. Panci and P. D. Serpico, Nucl. Phys. B 840 (2010) 284 [arXiv:0912.0663 [astro-ph.CO]].

[33] G. D. Kribs and I. Z. Rothstein, Phys. Rev. D 55, 4435 (1997) [Erratum-ibid. D 56, 1822 (1997)] [hep-ph/9610468].

[34] H. Yuksel and M. D. Kistler, Phys. Rev. D 78, 023502 (2008) [arXiv:0711.2906 [astro-ph]].

[35] J. A. R. Cembranos and L. E. Strigari, Phys. Rev. D 77, 123519 (2008) [arXiv:0801.0630 [astro-ph]].

[36] A. Boyarsky and O. Ruchayskiy, arXiv:0811.2385 [astro-ph]. 
[37] G. Bertone, W. Buchmuller, L. Covi and A. Ibarra, JCAP 0711 (2007) 003 [arXiv:0709.2299 [astro-ph]].

[38] A. Boyarsky, J. Nevalainen and O. Ruchayskiy, Astron. Astrophys. 471 (2007) 51 [astro-ph/0610961].

[39] A. Boyarsky, J. W. den Herder, A. Neronov and O. Ruchayskiy, Astropart. Phys. 28 (2007) 303 [astro-ph/0612219].

[40] A. Boyarsky, D. Malyshev, A. Neronov and O. Ruchayskiy, Mon. Not. Roy. Astron. Soc. 387 (2008) 1345 [arXiv:0710.4922 [astro-ph]].

[41] G. A. Gómez-Vargas, M. Fornasa, F. Zandanel, A. J. Cuesta, C. Munoz, F. Prada and G. Yepes, JCAP 1202 (2012) 001 [arXiv:1110.3305 [astro-ph.HE]].

[42] F. W. Stecker and A. J. Tylka, Astrophys. J. 343, 169 (1989).

[43] F. W. Stecker, Astrophys. J. 223, 1032 (1978).

[44] A. R. Pullen, R. -R. Chary and M. Kamionkowski, Phys. Rev. D 76 (2007) 063006 [Erratum-ibid. D 83 (2011) 029904] [astro-ph/0610295].

[45] E. Masso and R. Toldra, Phys. Rev. D 60 (1999) 083503 [astro-ph/9903397].

[46] K. Abazajian, G. M. Fuller and W. H. Tucker, Astrophys. J. 562 (2001) 593 [astro-ph/0106002].

[47] A. Boyarsky, A. Neronov, O. Ruchayskiy and M. Shaposhnikov, Mon. Not. Roy. Astron. Soc. 370 (2006) 213 [astro-ph/0512509].

[48] A. Kusenko, Phys. Rept. 481 (2009) 1 [arXiv:0906.2968 [hep-ph]].

[49] A. Palazzo, D. Cumberbatch, A. Slosar and J. Silk, Phys. Rev. D 76, 103511 (2007) [arXiv:0707.1495 [astro-ph]]. 
[50] A. Boyarsky, A. Neronov, O. Ruchayskiy and M. Shaposhnikov, Phys. Rev. D 74 (2006) 103506 [astro-ph/0603368].

[51] S. Riemer-Sorensen, S. H. Hansen and K. Pedersen, Astrophys. J. 644 (2006) L33 [astro-ph/0603661].

[52] C. R. Watson, J. F. Beacom, H. Yuksel and T. P. Walker, Phys. Rev. D 74 (2006) 033009 [astro-ph/0605424].

[53] K. N. Abazajian, M. Markevitch, S. M. Koushiappas and R. C. Hickox, Phys. Rev. D 75 (2007) 063511 [astro-ph/0611144].

[54] H. Yuksel, J. F. Beacom and C. R. Watson, Phys. Rev. Lett. 101, 121301 (2008) [arXiv:0706.4084 [astro-ph]].

[55] A. Boyarsky, A. Neronov, O. Ruchayskiy, M. Shaposhnikov and I. Tkachev, Phys. Rev. Lett. 97, 261302 (2006) [astro-ph/0603660].

[56] A. Boyarsky, O. Ruchayskiy and M. Markevitch, Astrophys. J. 673, 752 (2008) [astro-ph/0611168].

[57] A. Boyarsky, O. Ruchayskiy and M. Shaposhnikov, Ann. Rev. Nucl. Part. Sci. 59, 191 (2009) [arXiv:0901.0011 [hep-ph]].

[58] J. F. Navarro, C. S. Frenk and S. D. M. White, Astrophys. J. 462, 563 (1996) [astro-ph/9508025].

[59] J. F. Navarro, C. S. Frenk and S. D. M. White, Astrophys. J. 490 (1997) 493 [astro-ph/9611107].

[60] S. Kazantzidis, L. Mayer, C. Mastropietro, J. Diemand, J. Stadel and B. Moore, Astrophys. J. 608, 663 (2004) [astro-ph/0312194].

[61] J. N. Bahcall and R. M. Soneira, Astrophys. J. Suppl. 44 (1980) 73. 
[62] J. Einasto Trudy Inst. Astrofiz. Alma-Ata 5 (1965) 87.

[63] D. E. Gruber, J. L. Matteson, L. E. Peterson and G. V. Jung, astro-ph/9903492.

[64] L. Bouchet, E. Jourdain, J. P. Roques, A. Strong, R. Diehl, F. Lebrun and R. Terrier, arXiv:0801.2086 [astro-ph].

[65] Kappadath, S. C. 1998, Ph. D. Thesis, University of New Hampshire, USA.

[66] A. W. Strong, I. V. Moskalenko and O. Reimer, astro-ph/0306346; A. W. Strong, I. V. Moskalenko and O. Reimer, Astrophys. J. 613, 962 (2004) [astro$\mathrm{ph} / 0406254]$.

[67] [Fermi-LAT Collaboration], Astrophys. J. 750, 3 (2012) [arXiv:1202.4039 [astroph.HE]].

[68] P. Ullio, L. Bergstrom, J. Edsjo and C. G. Lacey, Phys. Rev. D 66, 123502 (2002) [astro-ph/0207125].

[69] J. E. Taylor and J. Silk, Mon. Not. Roy. Astron. Soc. 339, 505 (2003) [astro$\mathrm{ph} / 0207299]$.

[70] N. Arkani-Hamed, D. P. Finkbeiner, T. R. Slatyer and N. Weiner, Phys. Rev. D 79 (2009) 015014 [arXiv:0810.0713 [hep-ph]].

[71] C. Cheung, J. T. Ruderman, L. -T. Wang and I. Yavin, Phys. Rev. D 80 (2009) 035008 [arXiv:0902.3246 [hep-ph]].

[72] D. E. Morrissey, D. Poland and K. M. Zurek, JHEP 0907, 050 (2009) [arXiv:0904.2567 [hep-ph]].

[73] J. T. Ruderman and T. Volansky, JHEP 1002 (2010) 024 [arXiv:0908.1570 [hep$\mathrm{ph}]]$.

[74] B. Holdom, Phys. Lett. B 166 (1986) 196. 
[75] P. Galison and A. Manohar, Phys. Lett. B 136 (1984) 279.

[76] J. T. Ruderman and T. Volansky, arXiv:0907.4373 [hep-ph].

[77] T. Cohen, D. J. Phalen, A. Pierce and K. M. Zurek, Phys. Rev. D 82, 056001 (2010) [arXiv:1005.1655 [hep-ph]].

[78] J. Mardon, Y. Nomura, D. Stolarski and J. Thaler, JCAP 0905, 016 (2009) [arXiv:0901.2926 [hep-ph]].

[79] J. D. Bjorken, R. Essig, P. Schuster and N. Toro, Phys. Rev. D 80, 075018 (2009) [arXiv:0906.0580 [hep-ph]].

[80] S. Andreas, C. Niebuhr and A. Ringwald, Phys. Rev. D 86 (2012) 095019 [arXiv:1209.6083 [hep-ph]].

[81] S. Abrahamyan et al. [APEX Collaboration], Phys. Rev. Lett. 107 (2011) 191804 [arXiv:1108.2750 [hep-ex]].

[82] H. Merkel et al. [A1 Collaboration], Phys. Rev. Lett. 106 (2011) 251802 [arXiv:1101.4091 [nucl-ex]].

[83] B. Aubert et al. [BABAR Collaboration], Phys. Rev. Lett. 103 (2009) 081803 [arXiv:0905.4539 [hep-ex]].

[84] M. Pospelov, Phys. Rev. D 80 (2009) 095002 [arXiv:0811.1030 [hep-ph]].

[85] H. An, M. Pospelov and J. Pradler, Phys. Lett. B 725 (2013) 190 [arXiv:1302.3884 [hep-ph]].

[86] J. Redondo and G. Raffelt, JCAP 1308 (2013) 034 [arXiv:1305.2920 [hep-ph]].

[87] J. Redondo and M. Postma, JCAP 0902 (2009) 005 [arXiv:0811.0326 [hep-ph]]. 
[88] J. L. Hewett, H. Weerts, R. Brock, J. N. Butler, B. C. K. Casey, J. Collar, A. de Govea and R. Essig et al., arXiv:1205.2671 [hep-ex].

[89] R. E. Shrock, Nucl. Phys. B 206, 359 (1982).

[90] O. Ruchayskiy and A. Ivashko, JHEP 1206, 100 (2012) [arXiv:1112.3319 [hep$\mathrm{ph}]]$.

[91] S. Dodelson and L. M. Widrow, Phys. Rev. Lett. 72 (1994) 17 [hep-ph/9303287].

[92] A. Kusenko, Phys. Rev. Lett. 97 (2006) 241301 [hep-ph/0609081].

[93] K. Petraki and A. Kusenko, Phys. Rev. D 77 (2008) 065014 [arXiv:0711.4646 [hep-ph]].

[94] A. Y. Smirnov and R. Zukanovich Funchal, Phys. Rev. D 74, 013001 (2006) [hep-ph/0603009].

[95] A. D. Dolgov and F. L. Villante, Nucl. Phys. B 679, 261 (2004) [hep$\mathrm{ph} / 0308083]$.

[96] K. Kainulainen, J. Maalampi and J. T. Peltoniemi, Nucl. Phys. B 358, 435 (1991).

[97] T. Moroi, H. Murayama and M. Yamaguchi, Phys. Lett. B 303, 289 (1993).

[98] T. Moroi, hep-ph/9503210.

[99] F. Takayama and M. Yamaguchi, Phys. Lett. B 485, 388 (2000) [hep$\mathrm{ph} / 0005214]$.

[100] G. Moreau and M. Chemtob, Phys. Rev. D 65, 024033 (2002) [hep-ph/0107286].

[101] W. Buchmuller, L. Covi, K. Hamaguchi, A. Ibarra and T. Yanagida, JHEP 0703, 037 (2007) [hep-ph/0702184 [HEP-PH]]. 
[102] L. J. Hall, K. Jedamzik, J. March-Russell and S. M. West, JHEP 1003 (2010) 080 [arXiv:0911.1120 [hep-ph]].

[103] C. Cheung, G. Elor and L. Hall, Phys. Rev. D 84, 115021 (2011) [arXiv:1103.4394 [hep-ph]].

[104] L. J. Hall, J. T. Ruderman and T. Volansky, arXiv:1302.2620 [hep-ph].

[105] P. Langacker, Boca Raton, USA: CRC Pr. (2010) 663 p

[106] J. F. Beacom, N. F. Bell and G. Bertone, Phys. Rev. Lett. 94 (2005) 171301 [astro-ph/0409403].

[107] B. S. Hensley, V. Pavlidou and J. M. Siegal-Gaskins, MNRAS, 433, 591 (2013) [arXiv:1210.7239 [astro-ph.CO]]. 


\section{CHAPTER VII}

\section{Dissecting the Gamma-Ray Background in Search of Dark Matter}

Several classes of astrophysical sources contribute to the approximately isotropic gamma-ray background measured by the Fermi Gamma-Ray Space Telescope. In this paper, we use Fermi's catalog of gamma-ray sources (along with corresponding source catalogs at infrared and radio wavelengths) to build and constrain a model for the contributions to the extragalactic gamma-ray background from astrophysical sources, including radio galaxies, star-forming galaxies, and blazars. We then combine our model with Fermi's measurement of the gamma-ray background to derive constraints on the dark matter annihilation cross section, including contributions from both extragalactic and galactic halos and subhalos. The resulting constraints are competitive with the strongest current constraints from the Galactic Center and dwarf spheroidal galaxies. As Fermi continues to measure the gamma-ray emission from a greater number of astrophysical sources, it will become possible to more tightly

Written in collaboration with Ilias Cholis and Dan Hooper. Originally published as Dissecting

the Gamma-Ray Background in Search of Dark Matter, JCAP 1402, 014 (2014). arXiv:1312.0608 [hep-ph], MCTP-13-40, FERMILAB-PUB-13-546-A. 
constrain the astrophysical contributions to the extragalactic gamma-ray background. We project that with 10 years of data, Fermi's measurement of this background combined with the improved constraints on the astrophysical source contributions will yield a sensitivity to dark matter annihilations that exceeds the strongest current constraints by a factor of $\sim 5-10$

\section{$7.1 \quad$ Introduction}

The diffuse and approximately isotropic gamma-ray background was first detected by the SAS-2 satellite [1], and later confirmed by EGRET [2] and the Fermi GammaRay Space Telescope [3]. This emission has long been speculated to be the product of a large number of unresolved sources, such as active galactic nuclei [4-13] or starforming galaxies [14-17]. It was also suggested that a portion of this background could be the result of annihilating dark matter particles [18-20].

With the wealth of new information brought forth by Fermi, a much more concrete and detailed picture for the origin of the extragalactic gamma-ray background (EGB) has emerged. ${ }^{1}$ In particular, the large catalog of blazars observed by Fermi [21] has been used to construct detailed luminosity functions and redshift distributions for the populations of flat-spectrum radio quasars (FSRQs) and BL Lac objects. This information, as well as the degree of small-scale anisotropy observed by Fermi [22], supports the conclusion that unresolved blazars contribute only $\sim 20 \%$ of the EGB [2326]. Fermi's detection of gamma-ray emission from both star-forming galaxies [27] and radio galaxies [28], combined with the observed correlations between emission

\footnotetext{
${ }^{1}$ Although we will use the phrase "extragalactic gamma-ray background" throughout this paper to describe the approximately isotropic emission that is observed, we do not intend to imply that no galactic sources could contribute to this flux. If distributed sufficiently isotropically across the sky, a population of faint galactic sources would be difficult to separate from the extragalactic background. Despite the recent detection of small scale anisotropies [22], this background is also sometimes referred to as the isotropic gamma-ray background.
} 

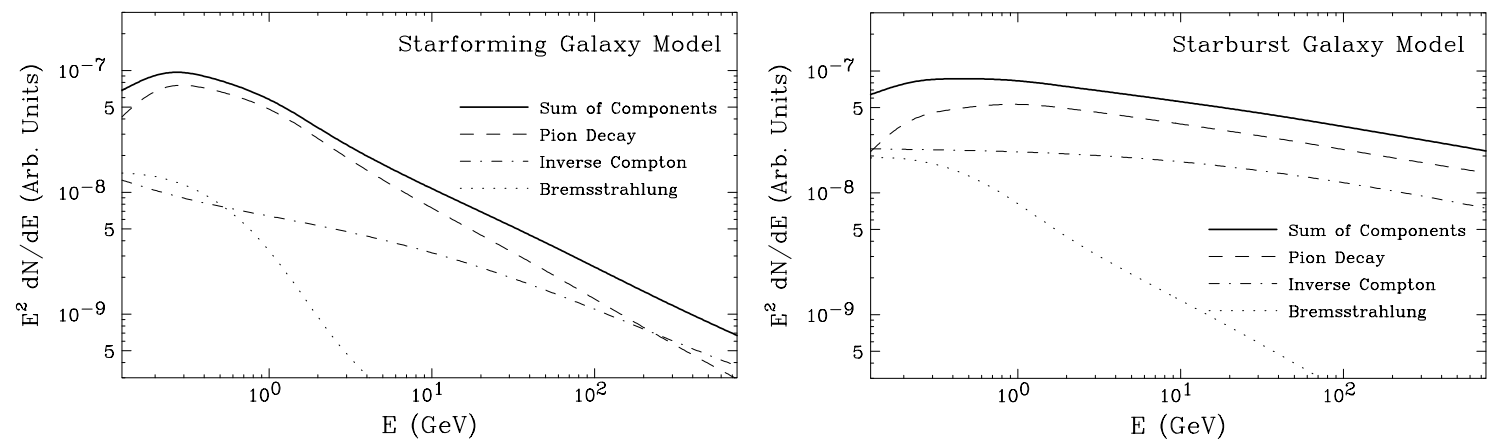

Figure 7.1: The models used in our analysis to describe the spectral shape of the gamma-ray emission from Milky Way-like star-forming galaxies (left) and much higher luminosity starburst galaxies (right), neglecting attenuation from the cosmic infrared background. See text for details.

at gamma-ray and infrared and radio wavelengths, has revealed that these source classes each contribute significantly to the EGB. Taken together, the emission from unresolved blazars, star-forming galaxies, and radio galaxies is likely to make up the majority of the observed EGB, and could plausibly constitute the entirety of this background (see, for example, the combinations presented in Refs. [29] and [30], or the discussion in Ref. [31]). Given the uncertainties in the characteristics of these source populations, however, there remains room for not-insignificant contributions from other sources, such as merging galaxy clusters [32-34], cascades generated in the propagation of ultra-high energy cosmic rays $[35,36]$, or annihilating or decaying dark matter.

In this paper, we construct an empirically based model for the contributions to the EGB from star-forming galaxies, radio galaxies, FSRQs, and BL Lac objects, and we compare this model to the observed spectrum of the EGB. We then make use of this model to derive upper limits on the contribution from dark matter, and on the corresponding annihilation cross sections. We find that the resulting dark matter constraints are competitive with those derived from observations of the Galactic Center [37] and dwarf spheroidal galaxies [38,39]. Furthermore, as Fermi continues 
to detect and characterize the gamma-ray emission from an ever larger number of sources, it will become increasingly possible to tightly constrain the various astrophysical contributions to the EGB. We project that with 10 years of data, Fermi's measurement of the EGB, combined with the expected constraints on the astrophysical source contributions, will yield a sensitivity to dark matter annihilations that exceeds current constraints by a factor of $\sim 5-10$. Such a result could plausibly represent the strongest constraint on the dark matter annihilation cross section by the end of the Fermi mission.

The remainder of this paper is structured as follows. In Sec. 7.2, we discuss contributions to the EGB from a variety of astrophysical sources, including star-forming galaxies, radio galaxies, blazars, cascades induced by ultra-high energy cosmic rays, and millisecond pulsars. We describe and constrain a model for this astrophysical emission, and find that the combination of these sources could account for the entirety of the EGB, although with significant statistical and systematic uncertainties. In Sec. 7.3, we calculate the contribution to the EGB from dark matter annihilations, including extragalactic halos and subhalos, and the halo and subhalos of the Milky Way. In Sec. 7.4, we use these results to derive constraints on the dark matter annihilation cross section. In Sec. 7.5, we make projections for Fermi's future sensitivity to annihilating dark matter. Finally, in Sec. 7.6, we summarize our results and conclusions.

\subsection{Astrophysical Contributions to the Diffuse Gamma-Ray Background}

In this section, we discuss several astrophysical contributions to the EGB, and constrain their spectral shapes and normalizations. Taken together, we find that the combination of emission from star-forming galaxies, radio galaxies, FSRQs and 
BL Lac objects likely makes up the majority of the EGB, although with significant uncertainties.

\subsubsection{Star-Forming Galaxies}

Although few galaxies (excluding those with active nuclei) are bright enough to be detected by Fermi as individual sources, they are very numerous and may collectively contribute significantly to the EGB $[15-17,31]$. Galaxies produce and contain cosmic rays, which generate gamma-rays through pion decay, inverse Compton, and bremsstrahlung processes. The intensity and spectrum of this emission is expected to depend on the star formation history of the galaxy in question. To date, Fermi has reported the detection of only nine individual galaxies, four of which reside within the Local Group (the SMC, LMC, M31, and Milky Way) and five of which are more distant (NGC 253, M82, NGC 4945, NGC 1068 and Circinus) [27,40-42]. Additionally, M82 and NGC 253 have been observed at very high-energies by ground-based gammaray telescopes $[43,44]$. Taking this information alone, it would be very difficult to produce a reliable model for the luminosity and redshift distribution of such sources. Fortunately, many more galaxies have been detected at infrared wavelengths [45], and the gamma-ray luminosities of the galaxies detected by Fermi have been shown to be highly correlated with the corresponding radio and infrared emission. In particular, Ref. [27] reports the following relationship between the emission in the $0.1-100 \mathrm{GeV}$ and 8-1000 $\mu \mathrm{m}$ bands:

$$
\log \left(\frac{L_{0.1-100 \mathrm{GeV}}}{\mathrm{erg} / \mathrm{s}}\right)=\alpha \log \left(\frac{L_{8-1000 \mu \mathrm{m}}}{10^{10} L_{\odot}}\right)+\beta
$$

where $\alpha=1.17 \pm 0.07$ and $\beta=39.28 \pm 0.08$. Combining this observed correlation with the observed infrared luminosity function and redshift distribution of galaxies [45], it is possible to derive the gamma-ray luminosity function for this source population [27]. 


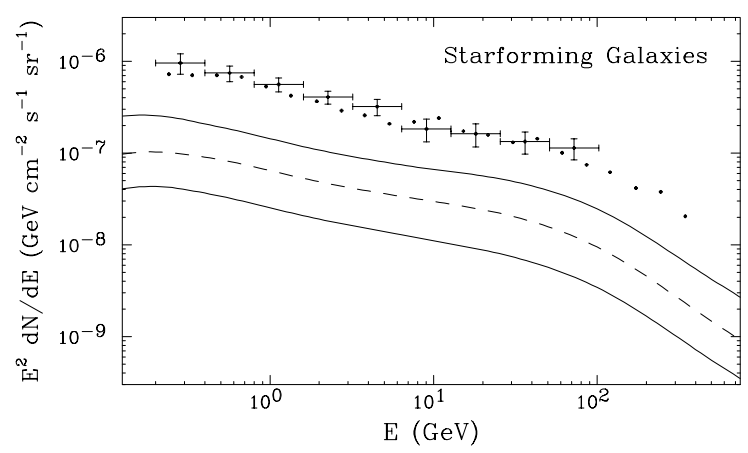

Figure 7.2: The estimated contribution to the EGB from star-forming galaxies (including starburst galaxies). The dashed curve represents the estimate derived using the central parameter values, while the solid lines are the $1 \sigma$ uncertainties around that prediction. The error bars denote the spectrum of the EGB as measured by Fermi [3], while the points without error bars are the central values of the Fermi's preliminary EGB analysis, currently in preparation and shown only for comparison [47]. See text for details.

To describe the spectral shape from this source population, we build a physical model for the gamma-ray spectra from star-forming and starburst galaxies, constrained to match the observed emission from such objects. In the left and right frames of Fig. 7.1, we plot the gamma-ray spectrum from a Milky Way-like starforming galaxy and a high luminosity starburst galaxy, respectively. In the starburst case, we select the pion, inverse Compton, and bremsstrahlung components to match the overall spectral index (above $1 \mathrm{GeV}$ ) of 2.2, as observed from individual starburst galaxies by Fermi [27]. In the Milky Way-like case, we normalized the various components (relatively) according to the model described in Ref. [46]. In calculating the contribution to the diffuse gamma-ray background, we describe the spectral shape from the combination of all star-forming galaxies (including starburst galaxies) as a weighted sum which is a function of a single parameter, $f$ :

$$
\frac{d N_{\gamma}}{d E_{\gamma}}=\left.f \frac{d N_{\gamma}}{d E_{\gamma}}\right|_{\text {star-forming }}+\left.(1-f) \frac{d N_{\gamma}}{d E_{\gamma}}\right|_{\text {starburst }}
$$

In Fig. 7.2, we show our estimate for the contribution to the EGB from star- 


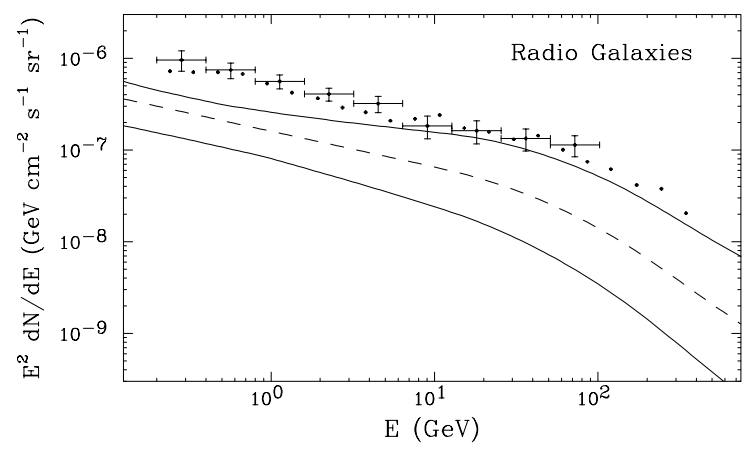

Figure 7.3: The estimated contribution to the EGB from radio galaxies (including both FRI and FRII galaxies). The dashed curve represents the estimate derived using the central parameter values, while the solid lines are the $1 \sigma$ uncertainties around that prediction. Error bars and points are as in Fig. 7.2. See text for details.

forming galaxies. The central (dashed) curve corresponds to the result found for $\alpha=1.17, \beta=39.28, f=0.5$, and the central value of the normalization of the infrared luminosity function [45]. To calculate the uncertainty for this contribution (solid, representing variations at the $1 \sigma$ level), we propagate the following uncertainties in these parameters: $\alpha=1.17 \pm 0.07, \beta=39.28 \pm 0.08, f=0.5 \times 10^{ \pm 0.20}$ (constrained such that $0<f<1$ ), and an overall uncertainty of $\pm 30 \%$ in the normalization of the infrared luminosity function. Taken together, we find that while star-forming galaxies are likely to produce only $\sim 10-15 \%$ of the extragalactic diffuse gamma-ray background, the related uncertainties are large, allowing for the possibility that their contribution could be more significant. Throughout this study, we adopt standard cosmological parameters $\left(\Omega_{\Lambda}=0.6817, \Omega_{M}=0.3183\right.$ [48]) and account for gamma-ray attenuation via pair-production with the cosmic infrared background $\left(\gamma+\gamma_{\mathrm{IR}} \rightarrow e^{+} e^{-}\right)$ using Ref. [49]'s "fiducial model" for the optical depth, $\tau\left(E_{\gamma}, z\right){ }^{2}$ 


\subsubsection{Radio Galaxies}

Radio galaxies are active galactic nuclei with relativistic jets that are not aligned with our line-of-sight. Within this context, Fanaroff-Riley (FR) type I and II radio galaxies are misaligned BL Lacs and FSRQs, respectively [50]. Although radio galaxies are much fainter than blazars, they are also much more numerous. As a result, gamma-ray emission from unresolved radio galaxies is expected to contribute significantly to the EGB $[28,31,51]$.

As with star-forming galaxies, only a small number (eleven at present) of radio galaxies have been detected at $\mathrm{GeV}$ energies [52]. But also like star-forming galaxies, a strong correlation has been observed between the $\mathrm{GeV}$ emission of radio galaxies and the emission produced at other wavelengths. In particular, the gamma-ray (0.1$10 \mathrm{GeV}$ ) and radio (5 GHz) emission from both FRI and FRII radio galaxies exhibit the following correlation [28]:

$$
\log \left(\frac{L_{0.1-10 \mathrm{GeV}}}{\operatorname{erg} / \mathrm{s}}\right)=A \log \left(\frac{L_{5 \mathrm{GHz}}}{\operatorname{erg} / \mathrm{s}}\right)+B,
$$

where $A=1.16 \pm 0.02$ and $B=-3.90 \pm 0.61$. We combine this observed correlation with the luminosity function and redshift distribution of radio galaxies as reported by Willott et al. [53] (which includes both FRI and FRII type galaxies) to generate a model for the resulting gamma-ray emission.

For the spectral shape of the gamma-ray emission from radio galaxies, we adopt a power-law with an index which we allow to vary from source-to-source around an average value. Using the ten spectral indices reported in Ref. [28], we find that a good fit is found for an average spectral index of $\Gamma=2.39 \pm 0.15$, with a source-tosource variation of $\sigma \approx 0.2$. In addition to the uncertainties on the spectral index and

\footnotetext{
${ }^{2}$ The "fiducial model" of Ref. [49] accounts for the evolution of the absorption efficiency of dust with redshift. If we had instead adopted their "fixed model" for the optical depth, this would have impact our limits by a factor of $\sim 2$ for dark matter masses greater than a few $\mathrm{TeV}$, and insignificantly for masses below $\sim 500 \mathrm{GeV}$.
} 
the radio-GeV correlation parameters, we include a $14 \%$ uncertainty in the overall normalization (corresponding to $\kappa=0.081 \pm 0.011$ in Ref. [28]). In Fig. 7.3, we show our estimate for the contribution of radio galaxies to the EGB, including the result derived using our central parameter values and the surrounding $1 \sigma$ uncertainty band.

\subsubsection{Blazars}

Blazars are by far the most numerous class of resolved extragalactic gamma-ray sources, and were long considered to be a leading candidate to generate the majority of the EGB. As the number of detected sources increased, however, it became apparent that unresolved blazars are unlikely to dominate this background. Taken together with the observed degree of anisotropy in the diffuse gamma-ray background at highlatitudes [22], blazars appear likely to account for only approximately $20 \%$ of the EGB [23-26].

To estimate the contribution from blazars to the EGB, we consider BL Lac objects and FSRQs independently. For each of these source classes, Fermi has resolved a large number of individual objects, making it possible to construct fairly reliable distributions of these sources in luminosity and redshift, without relying on correlations with emission at other wavelengths. Our method to estimate these contributions follows closely the works of Refs. [25, 26, 54], and we do not repeat the details here. In Fig. 7.4, we show the resulting contributions of BL Lacs and FSRQs to the EGB.

\subsubsection{Ultra-High Energy Cosmic Ray Propagation}

Ultra-high energy protons and nuclei scatter with the cosmic microwave and infrared backgrounds, leading to their attenuation and to the corresponding spectral feature known as the GZK cutoff $[55,56]$. Such interactions also initiate electromagnetic cascades. The energetic photons and electrons associated with such cascades undergo a rapid sequence of pair production and inverse Compton scattering events, 


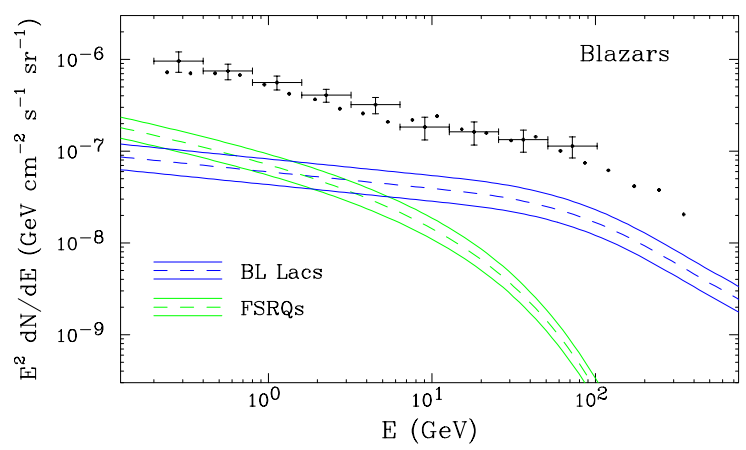

Figure 7.4: The estimated contribution to the EGB from blazars (BL Lacs and FSRQs). Once again, the dashed curve represents the estimate derived using the central parameter values, while the solid lines are the $1 \sigma$ uncertainties around that prediction. Error bars and points are as in Fig. 7.2. See text for details.

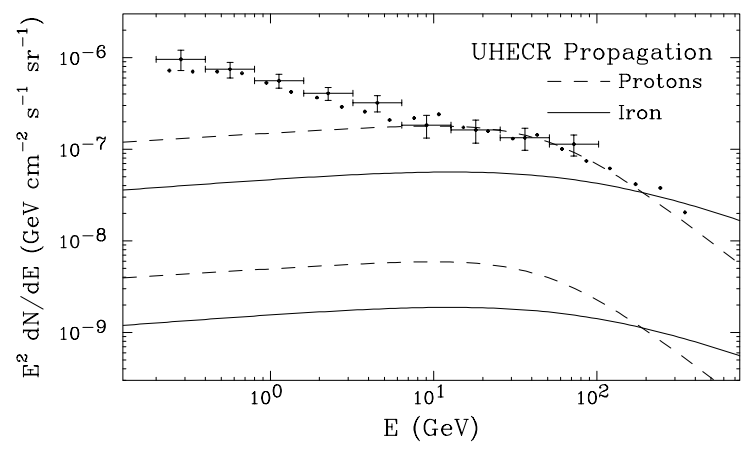

Figure 7.5: The estimated contribution to the EGB from the propagation of ultrahigh energy cosmic rays. The upper and lower sets of curves correspond to models with very strong source evolution and no source evolution, respectively [35]. Error bars and points are as in Fig. 7.2. See text for details. 
evolving rapidly downward in energy. The resulting spectrum of diffuse gamma-rays peaks at energies of $\sim 10-100 \mathrm{GeV}$, representing the approximate energy below which the universe is transparent to gamma-rays.

The spectrum of gamma-rays resulting from ultra-high energy cosmic ray (UHECR) propagation depends on a number of relatively unconstrained factors, including the redshift distribution of sources, the chemical composition of the UHECRs, the extragalactic magnetic field distribution, and the energy density of the cosmic radio background. As a result, very large uncertainties are associated with the overall flux of gamma-rays produced by such particles. The spectral shape of this contribution, in contrast, is less sensitive to these unknown factors. In Fig. 7.5 we show the contribution from UHECR propagation to the EGB for a few representative cases, as originally presented in Ref. [35] (see also, Ref. [36,57,58]). For each of the four curves shown, the injected cosmic ray spectrum is taken to consist purely of protons or iron nuclei, with a spectral index of 2.3, and with an exponential cutoff above $Z \times 10^{20.5}$ $\mathrm{eV}$ (where $Z=1$ for protons and 26 iron nuclei). The upper two curves assume a very strong source evolution, $n(z)=n_{0}(1+z)^{5}$, while the lower two curves adopt an unchanging source distribution with redshift, $n(z)=n_{0}$. These cases shown are rather extreme, and the true contribution from UHECR propagation is likely to fall somewhere within this range.

\subsubsection{Millisecond Pulsars}

Pulsars are rapidly spinning neutron stars which steadily convert their rotational kinetic energy into radiation, including potentially observable emission at radio and gamma-ray wavelengths. Due to their long lifetimes and expected spatial distribution, unresolved millisecond pulsars (MSPs), also known as recycled pulsars, have been considered as potential contributors to the high-latitude diffuse gamma-ray background [59] (see also Ref. [60]). 


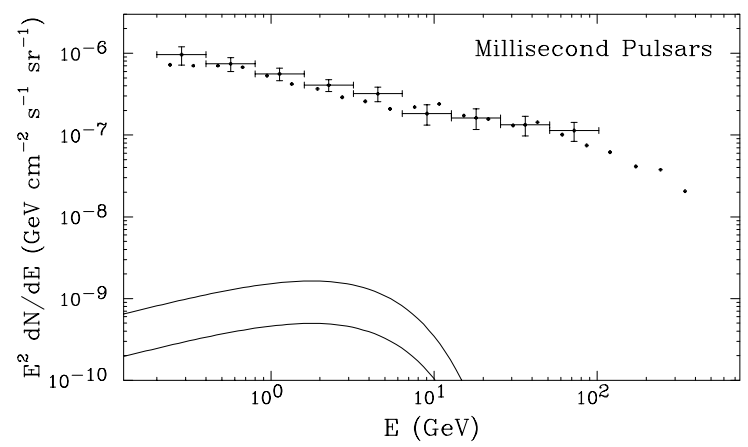

Figure 7.6: The estimated contribution to the high-latitude, diffuse gamma-ray background from millisecond pulsars. See text for details.

The Fermi Collaboration has detected gamma-ray emission from a total of 125 sources identified as pulsars, 47 of which have millisecond-scale periods [61]. Following Ref. [62], we build a spatial distribution and luminosity function model for galactic millisecond pulsars, constrained to account for the MSPs observed by Fermi without exceeding the total number of observed MSPs and currently unidentified gamma-ray sources. We also further constrain the spatial distribution to accommodate the distribution of such sources observed at radio frequencies [63]. Taken together, we find that MSPs are expected to account for only approximately $0.1 \%$ to $0.3 \%$ of the diffuse gamma-ray background above $1 \mathrm{GeV}$. This estimate is also compatible with constraints from Fermi's anisotropy measurement [64]. For details of the model used and its fit the observed MSP distribution, we direct the reader to Ref. [62]. For the spectral shape of the gamma-ray emission from unresolved MSPs, we adopt $d N_{\gamma} / d E_{\gamma} \propto E_{\gamma}^{-1.46} \exp \left(-E_{\gamma} / 3.3 \mathrm{GeV}\right)$, which provides a good fit to the spectra observed from individual MSPs [62].

In Fig. 7.6, we show our estimate for the contribution from millisecond pulsars to the diffuse gamma-ray background (integrated above $|b|>30^{\circ}$ ). The contribution has a negligible impact on our fits and limits, and thus we do not consider it further in this study. 


\subsubsection{Other Contributions}

There are several other contributions to the EGB which we will not explicitly include in this study. For the sake of completeness, we will briefly summarize some of these possible contributions here.

The mergers of galaxy clusters and other large scale structures can generate largescale collisionless shocks capable of accelerating electrons to highly relativistic energies. Through inverse Compton scattering with the cosmic microwave background, such electrons could potentially generate a non-negligible contribution to the diffuse gamma-ray background $[32,33]$. Assuming that $~ 5 \%$ of the thermal energy in such shocks is transferred to the acceleration of electrons, Ref. [32] finds that this mechanism could account for up to tens of percents of the diffuse gamma-ray background at energies above $\sim 10 \mathrm{GeV}$. In such a scenario, Fermi should be capable of detecting several merging clusters as gamma-ray sources $[32,34]$. Other estimates for this contribution are significantly lower $[33,65]$, however, and it is difficult to bound the expected contribution from this mechanism. As gamma-ray emission has not yet been detected from galaxy clusters [66-70], we do not include this contribution in our model at this time.

More local phenomena could also contribute to the diffuse gamma-ray background. In particular, interactions between cosmic rays and ionized hydrogen in the outer halo of the Milky Way could produce a diffuse flux of gamma-rays capable of accounting for $\sim 1-10 \%$ of the observed gamma-ray background [71]. Alternatively, interactions

of cosmic rays with debris in the Solar System's Oort Cloud could also contribute [72]. We do not include such local contributions in our calculations. 


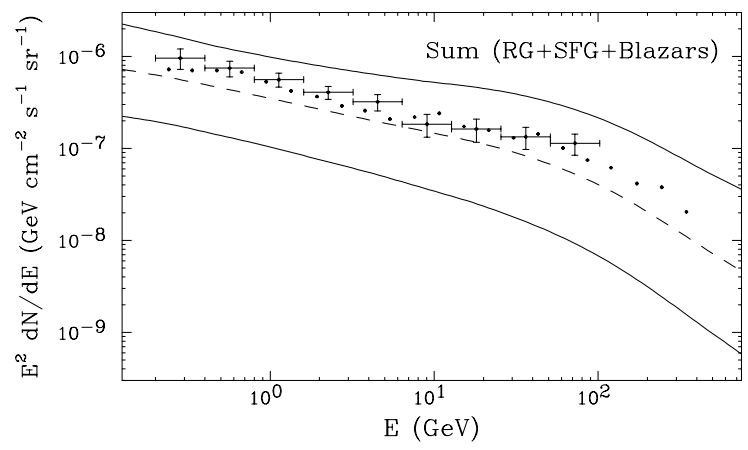

Figure 7.7: The estimated contribution to the EGB from the combination of radio galaxies, star-forming galaxies, and blazars (FSRQs and Bl Lacs). The dashed contour represents the prediction using central values for all model parameters. The solid contours are the $1 \sigma$ uncertainties around this prediction, after propagating all parameter uncertainties. Error bars and points are as in Fig. 7.2. See text for details.
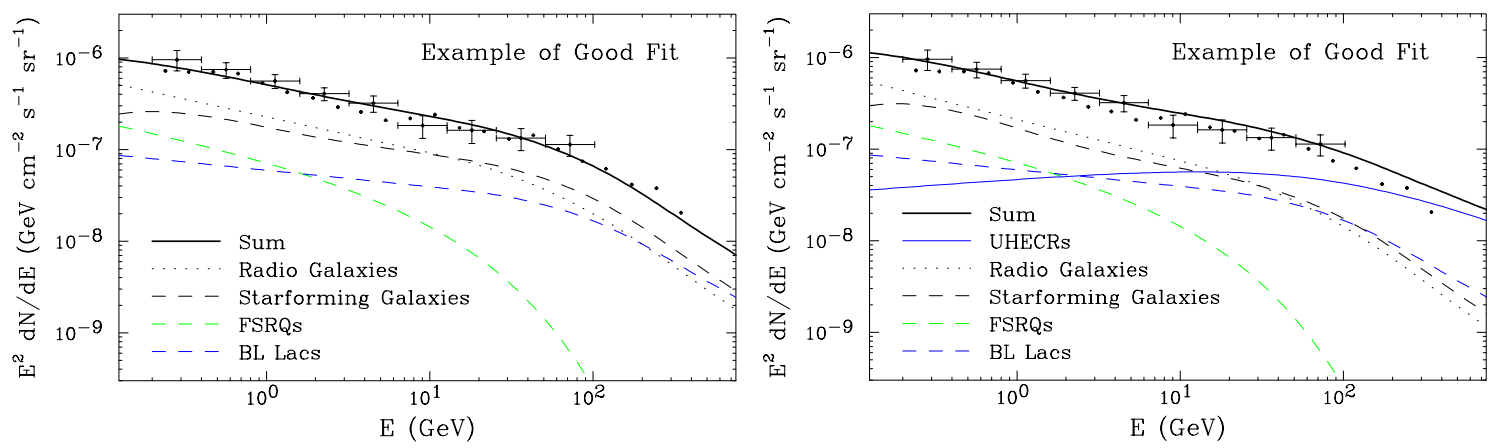

Figure 7.8: Two examples of viable models which provide a good fit to the observed EGB. See text for details. 


\subsubsection{The Combined Astrophysical Contribution to the Extragalactic Gamma- Ray Background}

In Fig. 7.7, we show the combined contributions to the EGB from radio galaxies, star-forming galaxies, FSRQs, and BL Lac objects. To evaluate a given model, we calculate the total chi-square $\left(\chi^{2}\right)$ corresponding to all of the parameter values, as described in Secs. 7.2.1, 7.2.2, and 7.2.3. The dashed curve in Fig. 7.7 represents the model with central values for each parameter, whereas the solid curves denote the range covered by all models which yield a $\chi^{2}$ that is within $1 \sigma$ of that found using the central parameter values. The result shown in Fig. 7.7 does not include in its fit the spectrum of the EGB as measured by Fermi.

Remarkably, we find that the entirety of the observed EGB can be accounted for by a combination of emission from radio galaxies, star-forming galaxies, and blazars. In Fig. 7.8 we show two specific examples of viable astrophysical models which provide a good fit to the observed EGB. In the left frame, we show a model with a negligible contribution from UHECR propagation, whereas the model in the right frame includes a significant contribution from UHECRs (corresponding to iron nuclei primaries, with strong source evolution). These models each yield excellent fits to the model parameters, as well as to Fermi's measurement of the EGB spectrum.

Although the astrophysical contributions included in our model are collectively able to account for the observed EGB, the uncertainties in the model remain fairly large and other contributions could also be significant. In the following sections, we calculate the isotropic gamma-ray spectrum from annihilating dark matter and include this contribution in our model of the EGB in order to derive upper limits on the corresponding annihilation cross section. 


\subsection{Gamma Rays from Annihilating Dark Matter}

If the dark matter consists of particles with weak-scale masses and cross sections, their annihilations could contribute significantly to the EGB. In this section, we examine the gamma-ray spectrum produced through dark matter particles annihilating in the halo of the Milky Way and throughout the universe. Throughout this section, we follow closely the approach of Ref. [73].

\subsubsection{The Extragalactic Contribution}

The intensity of the extragalactic gamma-ray background from dark matter annihilations is given by [73]:

$$
\begin{aligned}
\frac{d^{2} I_{\mathrm{eg}}\left(E_{\gamma}\right)}{d E_{\gamma} d \Omega}=\int \frac{d z}{H(z)} \frac{\langle\sigma v\rangle}{8 \pi m_{\mathrm{DM}}^{2}}(1+z)^{3} \frac{d N_{\gamma}}{d E_{\gamma}} e^{-\tau\left[E_{\gamma}(1+z), z\right]} \\
\quad \times \int d M \frac{d n(M, z)}{d M}\left[1+b_{\mathrm{sh}}(M, z)\right] \int d V \rho_{\text {host }}^{2}(r, M, z),
\end{aligned}
$$

where $d N_{\gamma} / d E_{\gamma}$ is the gamma-ray spectrum per annihilation (obtained from PPPC4DMID [74]), $\tau$ is the optical depth (again, using the model of Ref. [49]), $d n / d M$ is the halo mass function (which we tabulate with HMFcalc [75] using the model of Ref. [76]), and $\rho_{\text {host }}$ is the density profile of a given halo. As our benchmark model, we consider dark matter particles of mass $m_{\mathrm{DM}}$ and that annihilate to $b \bar{b}$. For this annihilation channel, the gamma-ray emission is dominated by the prompt photons, in contrast to contributions from inverse Compton scattering or bremsstrahlung emission, which we do not include in our calculations. In this work, we will take all host halos to have a density distribution defined by an NFW profile [77, 78]:

$$
\rho_{\mathrm{host}}=\frac{\rho_{s}}{x(1+x)^{2}}
$$


where $\rho_{s}$ is the scale density and $x=r / r_{s}$ is the distance from the center of the halo in units of the scale radius, $r_{s}$. We relate the scale and virial radii of a halo with the concentration, $c(M, z) \equiv r_{\text {vir }} / r_{s}$, as parameterized in Ref. [79]. The mass of a halo is related to its virial radius by:

$$
M=\frac{4 \pi}{3} r_{\mathrm{vir}}^{3} \Delta_{\mathrm{vir}}(z) \rho_{c}(z)
$$

where $\rho_{c}(z)$ is the cosmological dark matter density and $\Delta_{\text {vir }}(z)$ is the overdensity within the virial radius of a halo. This can be parameterized as $\Delta_{\text {vir }}(z)=18 \pi^{2}+$ $82 d-39 d^{2}$, where $d=\Omega_{M}(1+z)^{3} /\left[\Omega_{M}(1+z)^{3}+\Omega_{\Lambda}\right]-1$ [80]. In terms of these quantities, the scale density is given by:

$$
\rho_{s}=\frac{M}{4 \pi r_{s}^{3}}\left[\ln (1+c)-\frac{c}{1+c}\right]^{-1} .
$$

The density-squared integral in Eq. (7.4) can be written as

$$
\int d V \rho_{\text {host }}^{2}(r, M, z)=\frac{4 \pi r_{s}^{3} \rho_{s}^{2}}{3}\left[1-\frac{1}{(1+c)^{3}}\right]
$$

assuming that $\rho_{\text {host }}$ is described by an NFW profile.

The quantity $b_{\mathrm{sh}}$ accounts for the enhancement of the annihilation rate within a given halo as a result of substructures. As our default model, we consider the following parameterization for the boost factor:

$$
b_{\mathrm{sh}}(M, z)=110\left(M_{200}(M, z) / 10^{12} M_{\odot}\right)^{0.39}
$$

where $M_{200}$ is the mass of a halo contained within a region with an average density equal to 200 times the critical density (for a relationship between $M_{200}$ and $M_{\text {vir }}$, see Appendix C of Ref. [81]). 


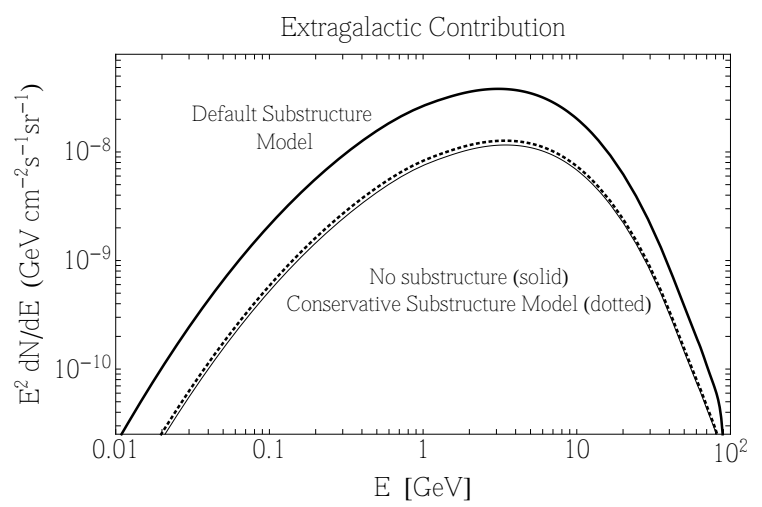

Figure 7.9: The extragalactic dark matter annihilation contribution to the EGB for a reference dark matter model $\left(m_{\mathrm{DM}}=100 \mathrm{GeV}\right.$, annihilating to $b \bar{b}$ with $\sigma v=3 \times 10^{-26} \mathrm{~cm}^{3} / \mathrm{s}$ ). The upper curve is the result using the substructure boost factor of Eq. 7.9, which is based on an extrapolation of numerical simulations. The dotted curve assumes a boost factor that is a factor of 30 lower than our default model. The lowest curve neglects the contribution from substructure entirely. See text for details.

The boost factor given in Eq. 7.9 was obtained from Ref. [82] (modified to account for all subhalos, including those outside of the volume containing mass $M_{200}$ [83]), and is based on the results of numerical simulations. To estimate the boost factor from such simulations, however, one must extrapolate to subhalos with masses well below the current resolution (the Aquarius simulation of Milky Way-like halos, for example, resolves subhalos with masses down to $\sim 3 \times 10^{4} M_{\odot}$ [84]). In particular, the result of Eq. 7.9 assumes that the subhalo mass function extends down to a minimum mass of $M_{\min }=10^{-6} M_{\odot}$, and that the mass-concentration relationship observed among very massive simulated subhalos can be extrapolated to much smaller subhalos. In regards to the minimum subhalo mass, the precise value of $M_{\text {min }}$ is determined by the temperature at which the dark matter particles decouple kinetically from the cosmic neutrino background. And while the value of $M_{\min }$ is model-dependent, typical dark matter candidates with masses and annihilation cross sections in the range of interest to this study generically yield minimum masses in the range of $M_{\min } \sim 10^{-9}-10^{-3} M_{\odot}[85,86]$. If we had increased the minimum subhalo mass assumed from $10^{-6}$ to $10^{-3}$ solar masses, for example, the boost factors 
would be reduced by a factor of $\sim 4$ relative to those given by Eq. 7.9. Of potentially greater importance, however, is the extrapolation of the subhalo mass-concentration relationship. If the concentrations of low mass subhalos are not as large as suggested by current extrapolations, the resulting boost factors could be very significantly reduced. As an example of the variation found in the literature, we note that the boost factors presented in Refs. [87-89] for galaxy-sized halos are between $\sim 8-30$ times smaller than those described in Eq. 7.9. With this in mind, we plot in Fig. 7.9 the contribution to the EGB from extragalactic dark matter annihilations, for a reference dark matter model $\left(m_{\mathrm{DM}}=100 \mathrm{GeV}\right.$, annihilating to $b \bar{b}$ with $\left.\sigma v=3 \times 10^{-26} \mathrm{~cm}^{3} / \mathrm{s}\right)$, and for three sets of assumptions regarding substructure. The upper curve is our default case (Eq. 7.9), while the lower dotted curve represents a more conservative case in which the boost factor is reduced by a factor of 30 . We also show a calculation which entirely neglects the contribution from substructure; this is shown as the lower solid line in Fig. 7.9. We note that the conservative case is almost indistinguishable from the case in which we neglect substructures entirely.

We briefly mention that our results are slightly different from those of Ref. [73], due to differences in our underlying assumptions. Firstly, the authors of Ref. [73] adopted a halo mass function based on an ellipsoidal collapse model, whereas we have instead adopted the model of Ref. [76]. Secondly, we have updated our cosmological parameters to include the recent results of the Planck experiment [48]. In Fig. 7.10, we show that the combined impact of these differences changes the overall normalization of the extragalactic dark matter signal by a factor of less than $\sim 20 \%$ relative to the results of Ref. [73]. 


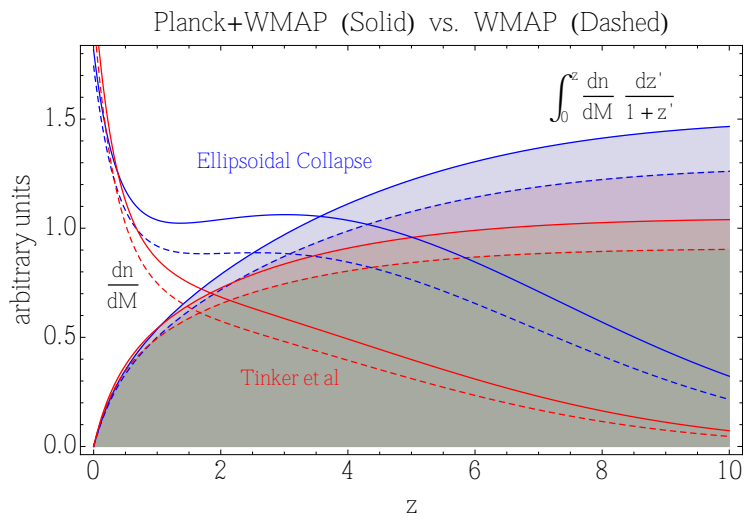

Figure 7.10: The halo mass function, $d n / d M$, and the integral of the $(1+z)^{-1}$ weighted halo mass function using the model of Tinker et al. [76] (adopted in our calculations) and the ellipsoidal collapse model adopted in Ref. [73]. We also show results using pre-Planck (dashed) and postPlanck (solid) values for the relevant cosmological parameters. These differences have only a modest impact on the contribution of dark matter annihilations to the extragalactic gamma-ray background.

\subsubsection{The Smooth Galactic Halo}

The angle-averaged intensity from dark matter annihilations in the halo of the Milky Way (neglecting substructures) is given by:

$$
\left\langle\frac{d I_{\mathrm{sm}}\left(E_{\gamma}\right)}{d E_{\gamma}}\right\rangle=\frac{\langle\sigma v\rangle}{2 m_{\mathrm{DM}}^{2}} \frac{d N_{\gamma}}{d E_{\gamma}} \frac{1}{\Omega_{e}} \int_{V_{*}} d V \frac{\rho^{2}(s, b, \ell)}{4 \pi s^{2}}
$$

where $s$ is the distance from the center of the halo, $b$ and $\ell$ are the direction in galactic coordinates, and $\Omega_{e}$ is the solid angle observed. We take the dark matter to be distributed according to an NFW profile, and we adopt parameters consistent with measurements: $r_{s}=21.5 \mathrm{kpc}, r_{\text {vir }}=258 \mathrm{kpc}$, and $M_{\text {vir }}=1.0 \times 10^{12} M_{\odot}[90]$. These parameters imply a local dark matter density of $\rho_{\odot} \approx 0.24 \mathrm{GeV} \mathrm{cm}^{-3}$, which is somewhat low compared to the more canonical estimates of $0.3-0.4 \mathrm{GeV} / \mathrm{cm}^{3}[91-$ 94]. If we had scaled up the dark matter density to a value in this range, the local annihilation rate would be further enhanced by a factor of $\sim 1.6-2.8$. 


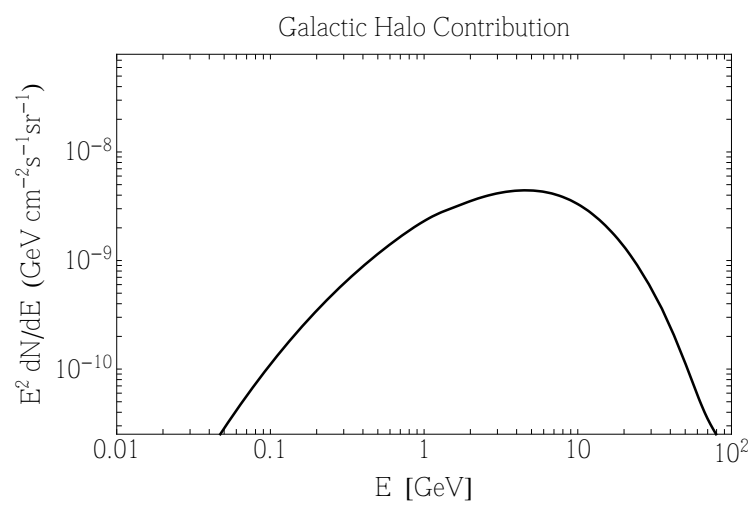

Figure 7.11: The contribution to the extragalactic gamma-ray background from dark matter annihilations in the smooth halo of the Milky Way, for a reference dark matter model $\left(m_{\mathrm{DM}}=100 \mathrm{GeV}\right.$, annihilating to $b \bar{b}$ with $\sigma v=$ $\left.3 \times 10^{-26} \mathrm{~cm}^{3} / \mathrm{s}\right)$. The result has been averaged over the following region of the sky: $0<\ell<2 \pi$ and $|b|>30^{\circ}$. See text for details.

The galactocentric radius is related to the distance along the line-of-sight by

$$
r^{2}=s^{2}+r_{\odot}^{2}-2 s r_{\odot} \cos b \cos \ell
$$

The solid angle of interest is described by $0 \leq \ell<2 \pi$ and $30^{\circ} \leq|b| \leq 90^{\circ}$.

In Fig. 7.11, we plot the contribution to the EGB from dark matter annihilations in the smooth component of the Milky Way's halo. Comparing this to the extragalactic contribution, we find that this component is likely to be subdominant, even for conservative assumptions pertaining to extragalactic substructure.

\subsubsection{Subhalos of the Milky Way}

Although the smooth halo of the Milky Way is predicted to provide no more than a subdominant contribution to the EGB, the intensity of gamma rays from dark matter annihilations in the subhalos of the Milky Way are expected to be comparable to the intensity of gamma rays from extragalactic structures. Each subhalo has a differential 
luminosity which is totally determined by its density profile:

$$
\frac{d L_{\gamma}}{d E_{\gamma}}=\frac{\langle\sigma v\rangle}{2 m_{\mathrm{DM}}^{2}} \frac{d N_{\gamma}}{d E_{\gamma}} \int d V \rho_{\mathrm{sub}}^{2}
$$

For a subhalo of mass, $M$, at a distance, $s$, along the line-of-sight, the photon intensity at earth is given by:

$$
\begin{aligned}
\frac{d i\left(E_{\gamma}, s, M\right)}{d E_{\gamma}} & =\frac{1}{4 \pi s^{2}} \frac{d L\left(E_{\gamma},\langle\sigma v\rangle, m_{\mathrm{DM}}, M\right)}{d E_{\gamma}} \\
& =\frac{1}{4 \pi s^{2}} \frac{b_{\mathrm{gs}}\langle\sigma v\rangle}{2 m_{\mathrm{DM}}^{2}} \frac{d N_{\gamma}}{d E_{\gamma}} \frac{M^{2}}{r_{s}(M)^{3}} g[c(M)]
\end{aligned}
$$

where $r_{s}$ is the scale radius of the subhalo and $b_{\mathrm{gs}}$ describes the contribution from substructure within each subhalo, which we set equal to 2, irrespective of mass [95]. The function $g[c(M)]$ arises from the integral over the volume of each satellite. For our default calculation, we set the subhalo concentrations following the approach of Ref. [96], where the subhalo is assumed to be initially described by an NFW profile which is then tidally stripped, leaving only a very compact and dark matterdominated object. In this case,

$$
g[c(M)]=\frac{1}{12 \pi}\left[1-\frac{1}{(1+c)^{3}}\right]\left[\ln (1+c)-\frac{c}{1+c}\right]^{-2}
$$

where $c$ is the concentration of the subhalo. In addition to our default assumptions, we also consider a more conservative scenario in which the contribution from galactic subhalos is suppressed by a factor of 30 relative to our default case, motivated by analogy to the extragalactic calculation.

The total intensity of gamma rays at Earth from dark matter particles annihilating in galactic subhalos is then given by integrating Eq. 7.13 over the distribution of Milky 


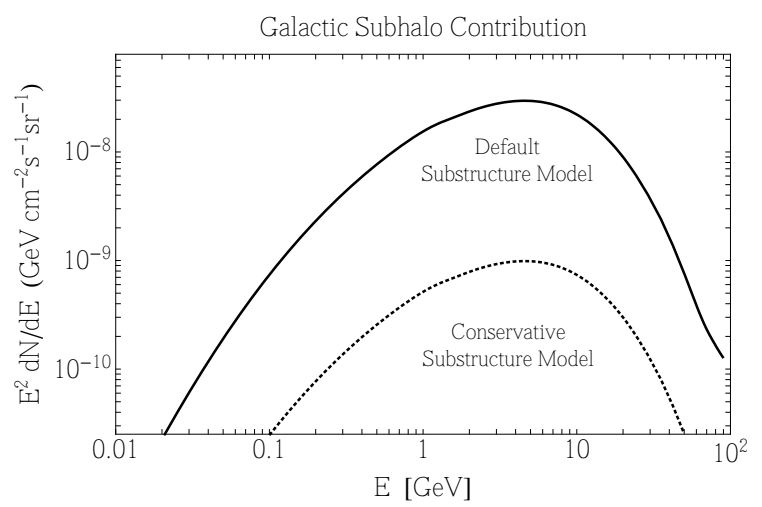

Figure 7.12: The contribution to the EGB from subhalos of the Milky Way, for a reference dark matter model $\left(m_{\mathrm{DM}}=100 \mathrm{GeV}\right.$, annihilating to $b \bar{b}$ with $\sigma v=3 \times 10^{-26} \mathrm{~cm}^{3} / \mathrm{s}$ ). The upper curve is the result using our default model, while the lower dotted curve is reduced by a factor of 30 relative to our default model. See text for details.

Way subhalos. Thus we have

$$
\frac{d I_{\mathrm{sub}}\left(E_{\gamma}\right)}{d E_{\gamma}}=\int d V d M \frac{d n_{\mathrm{sub}}(M, s, \ell, b)}{d M} \frac{d i\left(E_{\gamma}, s, M\right)}{d E_{\gamma}},
$$

where $\int d M d V\left(d n_{\mathrm{sub}} / d M\right)$ is the total number of subhalos in the Milky Way. We assume that the subhalo mass function, $d n_{\mathrm{sub}} / d M$, is given by the anti-biased case of Ref. [96], which is proportional to an Einasto profile with $\alpha_{E}=0.68$.

To compare to observations, we are interested in the angle-averaged intensity of gamma rays per unit energy over the entire galaxy. This is given by:

$$
\begin{aligned}
\left\langle\frac{d I_{\mathrm{sub}}\left(E_{\gamma}\right)}{d E_{\gamma}}\right\rangle= & \frac{1}{\Omega_{e}} \int_{M_{*}} \int_{V_{*}(M)} d V d M \times \\
& \times \frac{d n_{\mathrm{sub}}(M, s, \ell, b)}{d M} \frac{d i\left(E_{\gamma}, s, M\right)}{d E_{\gamma}},
\end{aligned}
$$

where $V_{*}$ is the volume beyond which satellites are not resolved. We consider subhalos with masses in the range of $10^{-6} M_{\odot} \leq M_{*} \leq 10^{10} M_{\odot}$, and assume that they are not resolvable beyond a distance of $s_{*}(M)=\sqrt{L(M) / 4 \pi F_{\text {sens }}}$, where $F_{\text {sens }}=2 \times$ $10^{-10} \mathrm{~cm}^{-2} \mathrm{sec}^{-1}[96]$ and $L(M)$ is the integral of Eq. (7.12) over all energy. 


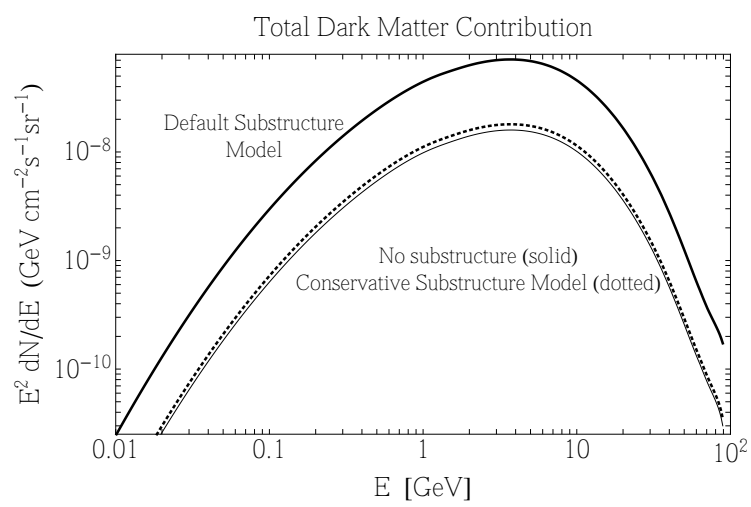

Figure 7.13: The total contribution from dark matter annihilations to the EGB, for a reference dark matter model $\left(m_{\mathrm{DM}}=100 \mathrm{GeV}\right.$, annihilating to $b \bar{b}$ with $\sigma v=3 \times 10^{-26} \mathrm{~cm}^{3} / \mathrm{s}$ ). The upper curve is the result using our default substructure model, while the lower dotted curve is reduces the contribution from substructure by a factor of 30 relative to our default model. See text for details.

In Fig. 7.12, we show the contribution to the EGB from galactic subhalos. For our default substructure model, this contribution is comparable to that from extragalactic dark matter annihilations. In our conservative substructure model, galactic subhalos are negligible compared to the EGB.

A summary of this section's results is given in Fig. 7.13. Here, we have plotted the combination of extragalactic, smooth galactic, and galactic subhalo contributions to the EGB. The upper solid curve adopts our default substructure model. The lower dotted and solid curves use our conservative substructure model or neglect substructure entirely, respectively. We note that contributions to the EGB from subhalos in the Milky Way and from extragalactic structure can be reduced significantly if low-mass halos and subhalos are not as highly concentrated as is suggested by extrapolations of simulations. The contribution from the smooth halo of the Milky Way, however, is significantly more robust. We also remind the reader that we have conservatively adopted a relatively low value of density of dark matter in the Milky Way (corresponding to a local density of $0.24 \mathrm{GeV} \mathrm{cm}^{-3}$ ). The more conservative models reduce the overall gamma ray flux from dark matter annihilations by only a 

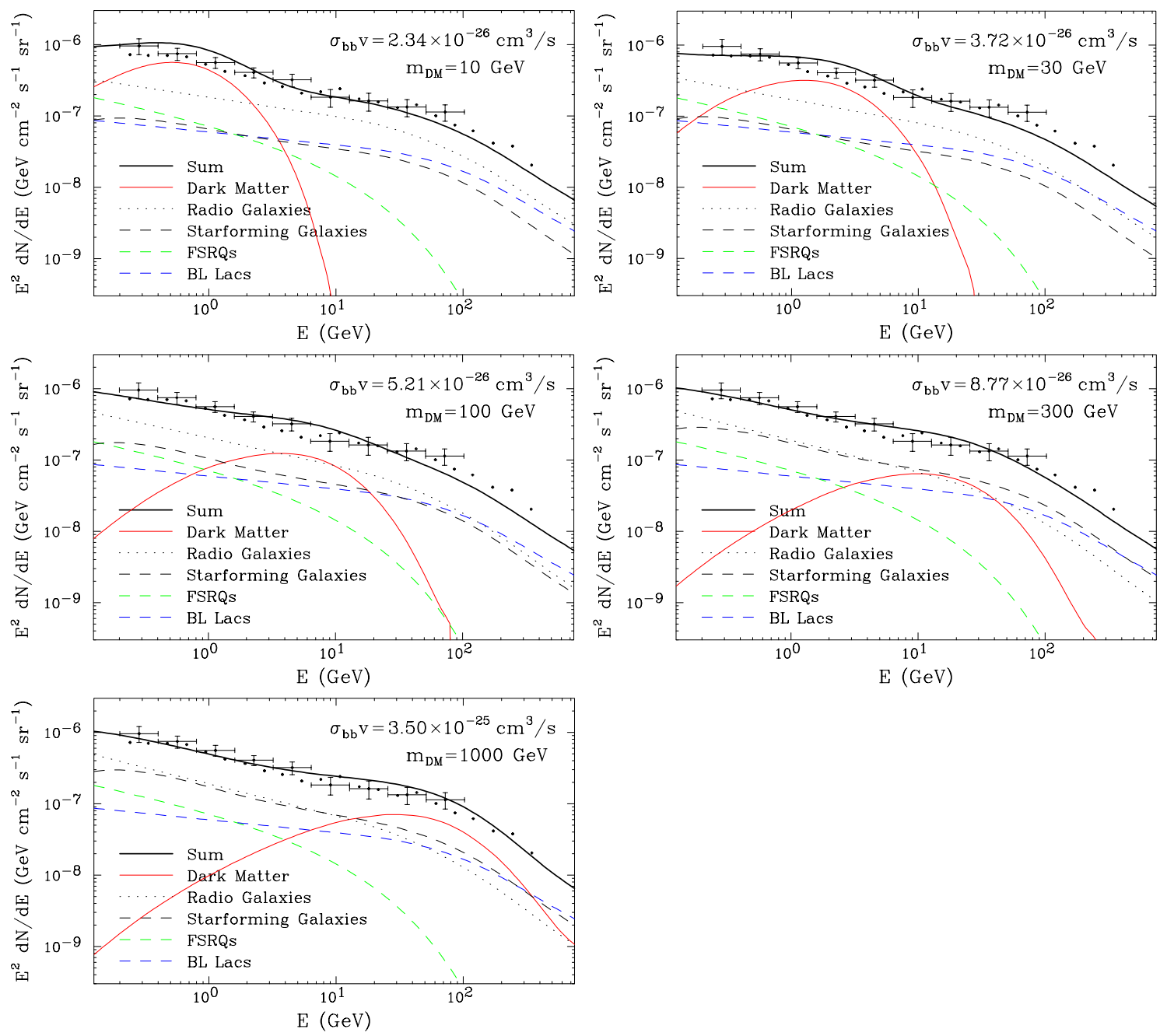

Figure 7.14: Our model of the EGB, including the largest allowed contribution from annihilating dark matter (at the 95\% CL). Here, we have adopted our default substructure model. In each case, we have marginalized over the parameters of our astrophysical model. See text for details.

factor of $\sim 4$ relative to our default model.

\subsection{Constraints on the Dark Matter Annihilation Cross Sec- tion}

In this section, we combine the results of Secs. 7.2 and 7.3 in order to place constraints on the contribution from annihilating dark matter to the EGB (for previous dark matter constraints derived from the EGB, see Refs. [73, 97-99]). 

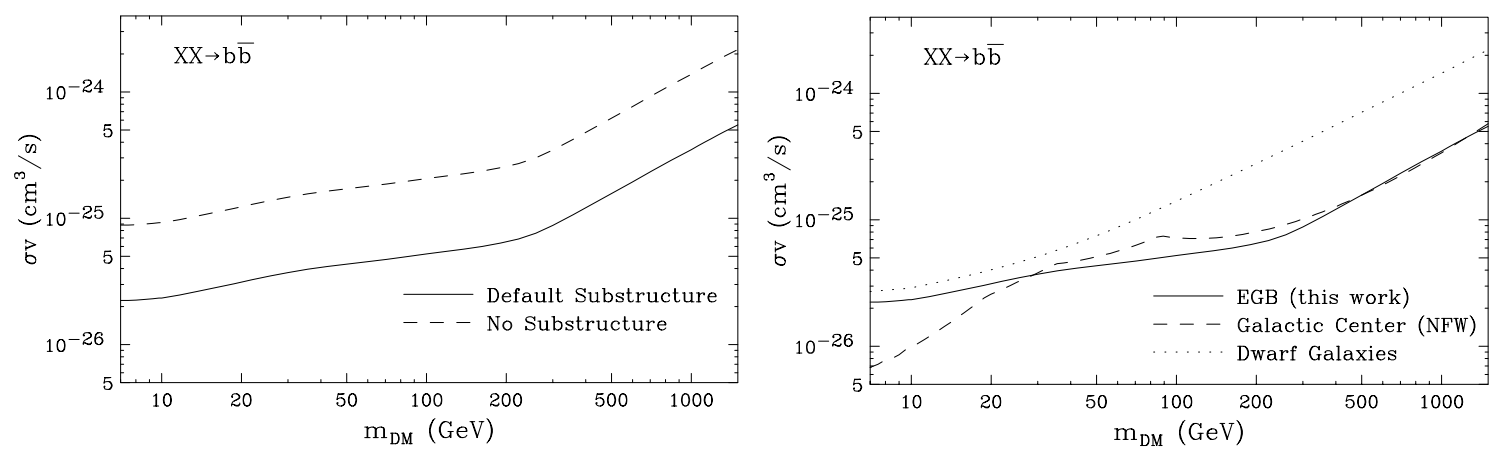

Figure 7.15: In the left frame, we show the limits (95\% CL) on the dark matter annihilation cross section derived in this study, using our default substructure model (solid), and neglecting substructure (dashes). In the right frame, we compare this result to the strongest existing constraints on the dark matter annihilation cross section from observations of the Galactic Center [37] and of dwarf spheroidal galaxies [38]. See text for details.

We begin by assessing the ability of a given model to fit the observed data. To do this, we construct a $\chi^{2}$ statistic:

$$
\chi^{2}=\sum_{i} \frac{\left(p_{i}-p_{i, 0}\right)^{2}}{\sigma_{p, i}^{2}}+\sum_{j} \frac{\left(d_{j}-d_{j, 0}\right)^{2}}{\sigma_{d, j}^{2}}
$$

where the first sum is performed over the astrophysical parameters of the model $\left(p_{i}\right)$, as described in Sec. 7.2, and the second sum is performed over the the error bars of the EGB spectrum as reported by the Fermi collaboration [3]. The quantities $\sigma_{p, i}$ and $\sigma_{d, j}$ represent the uncertainties in the astrophysical parameters and the errors in the measured spectrum, respectively. With no contribution from dark matter, our best model parameter set yields an overall value of $\chi^{2}=8.54 .^{3}$ This model includes contributions from radio galaxies, star-forming galaxies, FSRQs, and BL Lac objects, with uncertainties in the model parameters as described in Sec. 7.2.

To place limits on the dark matter annihilation cross section, we add a contribution

\footnotetext{
${ }^{3}$ Of this $\chi^{2}=8.54,4.62$ comes from the 9 degrees-of-freedom associated with Fermi's measurement of the EGB. The remaining 3.92 comes from the fit to our model of the contributions from radio galaxies, star-forming galaxies, and blazars. See Sec. 7.2 for details.
} 
from annihilations of dark matter (with a given mass and annihilation channel) to our model. We increase the value of the cross section until the best possible $\chi^{2}$ (marginalizing over all the parameters of the astrophysics model) increases by 2.71 over the best-fit with no dark matter component (corresponding to the $95 \%$ confidence level upper limits). In Fig. 7.14, we show the contributions to the EGB in models with the maximum allowed contribution from annihilating dark matter (assuming annihilations to exclusively to $b \bar{b}$ for five choices of the dark matter mass).

In the left frame of Fig. 7.15, we plot the upper limits on the dark matter annihilation cross section derived in this study. In the right frame, this result is compared to the limits obtained from observations of the Galactic Center [37] and of dwarf spheroidal galaxies [38]. For our default substructure model, the limits presented here are approximately as stringent as those derived from the Galactic Center (assuming an NFW profile). Our limits obtained neglecting contributions from substructure are comparably stringent to those derived from the Galactic Center assuming a profile with a kiloparsec-scale core [37]. And although the constraint from dwarf galaxies is somewhat less susceptible to astrophysical uncertainties than those derived from the EGB or Galactic Center, even for very conservative assumptions (i.e.. negligible contributions from substructure) the constraints derived here are as or more sensitive to dark matter particles with masses on the order of $100 \mathrm{GeV}$ or greater.

\subsection{Projections And Future Sensitivity}

As Fermi continues to collect data, its sensitivity to dark matter annihilation products in the EGB will increase due to two different sets of factors. Firstly, Fermi's measurement of the EGB itself will improve, reducing the errors on the corresponding spectrum and extending the measurement to higher energies. Secondly, with a larger data set, Fermi will detect $\mathrm{GeV}$ emission from a greater number of radio galaxies, star-forming galaxies, and blazars, and will characterize the emission from those 
sources already detected with greater precision. As it does so, the uncertainties in the contributions to the EGB from these sources classes will be reduced considerably.

To project the error bars on Fermi's future (after 10 total years of operation) measurement of the EGB, we take the preliminary spectrum (which is based on 44 months of data, and is shown in the left frame of Fig. 7.16 [47]) and further reduce the size of the error bars by a factor of $\sqrt{120 / 44} \approx 1.65$. Note that in this projection, we have not removed contributions from to-be-resolved blazars, in order to better facilitate comparisons between projected and current models and measurements. To project the improvement in the uncertainties of our astrophysical parameters (IR/radio correlation parameters, spectral indices, etc.), we reduce each error bar by the square root of time (relative to the amount of data that was used in the analysis of each source population). We conservatively do not account for any possible improvements in the uncertainties of the radio or IR luminosity functions when making our projections.

In each frame of Fig. 7.16, we show the projected uncertainties for an astrophysical model of the EGB after 10 years of Fermi data. In the left frame, we compare this to the preliminary Fermi (44 month) measurement of the EGB [47]. In the right frame, we compare this model to our projection for Fermi's measurement of the EGB with 10 years of data. Using this projection for the model parameters and EGB measurements, we repeat the procedure used in Sec. 7.4 to predict the constraints that Fermi should be able to place on the dark matter annihilation cross section after 10 years of observation. These projected constraints are shown in Fig. 7.17.

We note that the shape of the projected limits shown in Fig. 7.17 does not mimic that of the current constraints. The break in the projected limit at $m_{\mathrm{DM}} \sim 50 \mathrm{GeV}$ is the consequence of the error bars being fairly large below $E_{\gamma} \sim 1-2 \mathrm{GeV}$. If these error bars were as small as the error bars in the higher energy bins, no break would be evident and the constraints below $m_{\mathrm{DM}} \sim 50 \mathrm{GeV}$ would be significantly stronger. 

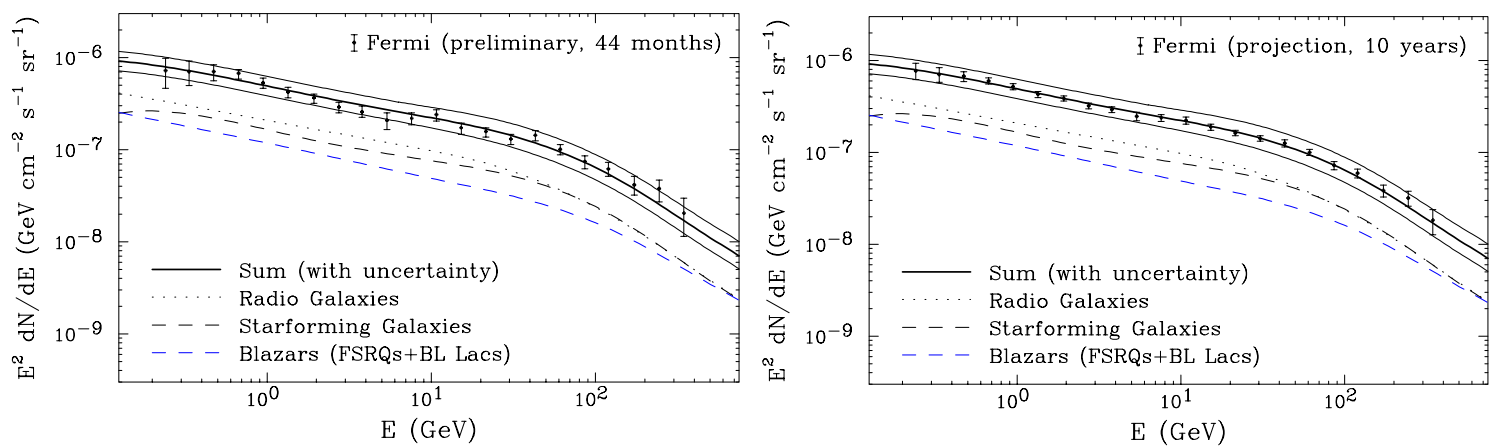

Figure 7.16: Projected uncertainties for an astrophysical model of the extragalactic gamma-ray background, after ten years of data from Fermi. In the left frame, we compare this model to the preliminary Fermi measurement [47], whereas in the right frame we compare it to the measurement projected with ten years of data. See text for details.

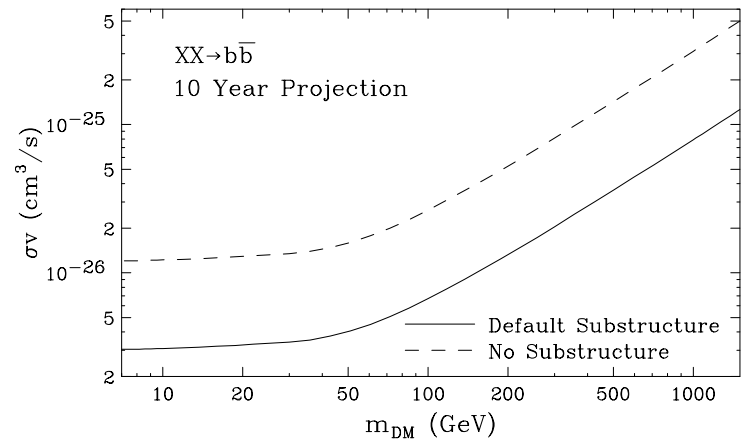

Figure 7.17: Our projected sensitivity to dark matter annihilation from Fermi measurements of the EGB after 10 years of operation, using the astrophysical model and projected error bars as shown in the right frame of Fig. 7.16. See text for details.

In the case of the current results shown in Fig. 7.15, the shape is further influenced by the especially low error bar near $9 \mathrm{GeV}$. This strengthens the bounds for dark matter masses near $m_{\mathrm{DM}} \sim 200 \mathrm{GeV}$.

\subsection{Summary and Conclusions}

The extragalactic gamma-ray background (EGB) as measured by the Fermi GammaRay Space Telescope contains contributions from a variety of astrophysical sources, including radio galaxies, star-forming galaxies, and blazars. Fermi observations of 
individual members of these source classes have been used to construct distribution functions for these populations in both luminosity and redshift. As Fermi collects more data, these distributions will become more tightly constrained, making it possible to determine their contributions to the EGB with increasing precision.

In this paper, we have constructed a model for the astrophysical contributions to the EGB, and used this model along with Fermi's measurement of the EGB to constrain the contribution from annihilating dark matter. Included in this calculation are contributions from dark matter annihilating in the halos and subhalos distributed throughout the universe, as well as that of the Milky Way's halo and subhalos. The limits on the dark matter's annihilation cross section that we derive in this study are competitive with those based on observations of the Galactic Center and dwarf spheroidal galaxies. In particular, adopting a substructure model based on the extrapolation of numerical simulations (our "default" model), the limits presented here are, for all masses, more stringent than those from dwarf galaxies, as recently published by the Fermi collaboration. If we conservatively neglect the contributions from subhalos, our limits become somewhat less stringent (by a factor of $\sim 4-5$ ) but are still competitive with those derived from dwarfs.

As Fermi collects more data, it will not only be capable of measuring the spectrum of the EGB with greater precision, but will also more stringently constrain the characteristics of the various astrophysical source populations that contribute to the EGB. As a result, we project that Fermi will ultimately be able to achieve a sensitivity to dark matter annihilation products in the EGB that exceeds current constraints by a factor of $\sim 5-10$. For our default substructure model, we project that the Fermi measurement of the EGB will ultimately be sensitive to dark matter with the canonical thermal annihilation cross section $\left(\sigma v=3 \times 10^{-26} \mathrm{~cm}^{3} / \mathrm{s}\right)$ for masses up to $\sim 400$ GeV. At the end of Fermi's mission, such limits will likely be the strongest constraints on the dark matter annihilation cross section, although constraints from cosmic-ray 
observations could in some cases be competitive and complementary [100-106].

Finally, we stress that dark matter searches making use of the EGB are complementary to those based on observations of the Galactic Center and dwarf galaxies. The main systematic error in searches involving the region of the Galactic Center arises from uncertainties in the distribution of dark matter in the Milky Way's inner halo. While the uncertainties faced here regarding dark matter substructure are of a comparable magnitude, they are independent of those issues pertaining to the Inner Galaxy. Furthermore, while the constraints derived from dwarf galaxies are likely more robust to systematic uncertainties than those based on either the Galactic Center or the EGB, they are also somewhat less stringent. As Fermi collects more data, all three of these search techniques will become significantly more powerful, and together will be able to test a wide range of models in which the dark matter consists of thermal relics with masses up to $\sim 400 \mathrm{GeV}$.

Acknowledgements: We would like to thank Shin'ichiro Ando and Andrew Hearin for helpful discussion. This work has been supported by the US Department of Energy and by the Kavli Institute for Cosmological Physics. IC and DH would like to thank the Aspen Center for Physics and the NSF Grant 1066293 for hospitality during the earlier stages of this project. 


\section{Bibliography}

[1] C. E. Fichtel, G. A. Simpson, and D. J. Thompson, Astrophys. J. 222, 833 (1978).

[2] P. Sreekumar et al. [EGRET Collaboration], Astrophys. J. 494, 523 (1998) [astro$\mathrm{ph} / 9709257]$.

[3] A. A. Abdo et al. [Fermi-LAT Collaboration], Phys. Rev. Lett. 104, 101101 (2010) [arXiv:1002.3603 [astro-ph.HE]].

[4] F. W. Stecker, M. H. Salamon and M. A. Malkan, Astrophys. J. 410, L71 (1993).

[5] P. Padovani, et al., MNRAS, 260, L21 (1993).

[6] M. H. Salamon and F. W. Stecker, Astrophys. J. 430, L21 (1994).

[7] F. W. Stecker and M. H. Salamon, Astrophys. J. 464, 600 (1996) [astro$\mathrm{ph} / 9601120]$.

[8] R. Mukherjee and J. Chiang, Astropart. Phys. 11, 213 (1999) [astro-ph/9902003].

[9] T. Narumoto and T. Totani, Astrophys. J. 643, 81 (2006) [astro-ph/0602178].

[10] P. Giommi, S. Colafrancesco, E. Cavazzuti, M. Perri and C. Pittori, Astron. Astrophys. 445, 843 (2006) [astro-ph/0508034].

[11] C. D. Dermer, Astrophys. J. 659, 958 (2007) [astro-ph/0605402].

[12] V. Pavlidou and T. M. Venters, Astrophys. J. 673, 114 (2008) [arXiv:0710.0002 [astro-ph]].

[13] Y. Inoue and T. Totani, Astrophys. J. 702, 523 (2009) [Erratum-ibid. 728, 73 (2011)] [arXiv:0810.3580 [astro-ph]].

[14] V. Pavlidou and B. D. Fields, Astrophys. J. 575, L5 (2002) [astro-ph/0207253]. 
[15] T. A. Thompson, E. Quataert and E. Waxman, Astrophys. J. 654, 219 (2006) [astro-ph/0606665].

[16] B. D. Fields, V. Pavlidou and T. Prodanovic, Astrophys. J. 722, L199 (2010) [arXiv:1003.3647 [astro-ph.CO]].

[17] R. Makiya, T. Totani and M. A. R. Kobayashi, Astrophys. J. 728, 158 (2011) [arXiv:1005.1390 [astro-ph.HE]].

[18] F. W. Stecker, Astrophys. J. 223, 1032 (1978).

[19] Y. -T. Gao, F. W. Stecker and D. B. Cline, Astron. Astrophys. 249, 1 (1991).

[20] P. Ullio, L. Bergstrom, J. Edsjo and C. G. Lacey, Phys. Rev. D 66, 123502 (2002) [astro-ph/0207125].

[21] M. Ackermann, M. Ajello, A. Allafort, et al., Astrophys. J. 743, 171 (2011).

[22] M. Ackermann et al. [Fermi LAT Collaboration], Phys. Rev. D 85, 083007 (2012) [arXiv:1202.2856 [astro-ph.HE]].

[23] A. Cuoco, E. Komatsu and J. M. Siegal-Gaskins, Phys. Rev. D 86, 063004 (2012) [arXiv:1202.5309 [astro-ph.CO]].

[24] J. P. Harding and K. N. Abazajian, JCAP 1211, 026 (2012) [arXiv:1206.4734 [astro-ph.HE]].

[25] [Fermi-LAT Collaboration], Astrophys. J. 720, 435 (2010) [arXiv:1003.0895 [astro-ph.CO]].

[26] M. Ajello, M. S. Shaw, R. W. Romani, C. D. Dermer, L. Costamante, O. G. King, W. Max-Moerbeck and A. Readhead et al., Astrophys. J. 751, 108 (2012) [arXiv:1110.3787 [astro-ph.CO]]. 
[27] M. Ackermann et al. [Fermi LAT Collaboration], Astrophys. J. 755, 164 (2012) [arXiv:1206.1346 [astro-ph.HE]].

[28] Y. Inoue, Astrophys. J. 733, 66 (2011) [arXiv:1103.3946 [astro-ph.HE]].

[29] J. M. Siegal-Gaskins, arXiv:1308.2228 [astro-ph.HE].

[30] M. Cavadini, R. Salvaterra and F. Haardt, arXiv:1105.4613 [astro-ph.CO].

[31] F. W. Stecker and T. M. Venters, Astrophys. J. 736, 40 (2011) [arXiv:1012.3678 [astro-ph.HE]].

[32] U. Keshet, E. Waxman, A. Loeb, V. Springel and L. Hernquist, Astrophys. J. 585, 128 (2003) [astro-ph/0202318].

[33] S. Gabici and P. Blasi, Astropart. Phys. 19, 679 (2003) [astro-ph/0211573].

[34] S. Gabici and P. Blasi, Astropart. Phys. 20, 579 (2004) [astro-ph/0306369].

[35] M. Ahlers and J. Salvado, Phys. Rev. D 84, 085019 (2011) [arXiv:1105.5113 [astro-ph.HE]].

[36] G. B. Gelmini, O. Kalashev and D. V. Semikoz, JCAP 1201, 044 (2012) [arXiv:1107.1672 [astro-ph.CO]].

[37] D. Hooper, C. Kelso and F. S. Queiroz, Astropart. Phys. 46, 55 (2013) [arXiv:1209.3015 [astro-ph.HE]].

[38] M. Ackermann et al. [Fermi-LAT Collaboration], arXiv:1310.0828 [astro-ph.HE].

[39] A. Abramowski et al. [HESS Collaboration], Astropart. Phys. 34, 608 (2011) [arXiv:1012.5602 [astro-ph.HE]].

[40] A. A. Abdo [Fermi LAT Collaboration], Astrophys. J. 709, L152 (2010) [arXiv:0911.5327 [astro-ph.HE]]. 
[41] B. C. Lacki, T. A. Thompson, E. Quataert, A. Loeb and E. Waxman, Astrophys. J. 734, 107 (2011) [arXiv:1003.3257 [astro-ph.HE]].

[42] M. Hayashida, L. Stawarz, C. C. Cheung, K. Bechtol, G. M. Madejski, M. Ajello, F. Massaro and I. V. Moskalenko et al., arXiv:1310.1913 [astro-ph.HE].

[43] V. A. Acciari, E. Aliu, T. Arlen, et al., Nature, 462, 770 (2009).

[44] F. Acero, F. Aharonian, A. G. Akhperjanian, et al., Science, 326, 1080 (2009).

[45] G. Rodighiero, M. Vaccari, A. Franceschini, et al., A\&A, 515, A8 (2010).

[46] A. W. Strong, T. A. Porter, S. W. Digel, G. Johannesson, P. Martin, I. V. Moskalenko and E. J. Murphy, Astrophys. J. 722, L58 (2010) [arXiv:1008.4330 [astro-ph.HE]].

[47] http://fermi.gsfc.nasa.gov/science/mtgs/symposia/2012/program/thu/ MAckermann.pdf

[48] P. A. R. Ade et al. [Planck Collaboration], arXiv:1303.5076 [astro-ph.CO].

[49] R. C. Gilmore, R. S. Somerville, J. R. Primack and A. Dominguez, arXiv:1104.0671 [astro-ph.CO].

[50] C. M. Urry and P. Padovani, Publ. Astron. Soc. Pac. 107, 803 (1995) [astroph/9506063].

[51] M. Di Mauro, F. Calore, F. Donato, M. Ajello and L. Latronico, arXiv:1304.0908 [astro-ph.HE].

[52] A. A. Abdo [Fermi LAT Collaboration], Astrophys. J. 720, 912 (2010).

[53] C. J. Willott, S. Rawlings, K. M. Blundell, M. Lacy and S. A. Eales, Mon. Not. Roy. Astron. Soc. 322, 536 (2001) [astro-ph/0010419]. 
[54] M. Ajello, R. W. Romani, D. Gasparrini, M. S. Shaw, J. Bolmer, G. Cotter, J. Finke and J. Greiner et al., arXiv:1310.0006 [astro-ph.CO].

[55] K. Greisen, Phys. Rev. Lett. 16, 748 (1966).

[56] G. T. Zatsepin and V. A. Kuzmin, JETP Lett. 4, 78 (1966) [Pisma Zh. Eksp. Teor. Fiz. 4, 114 (1966)].

[57] K. Murase, J. F. Beacom and H. Takami, JCAP 1208, 030 (2012) [arXiv:1205.5755 [astro-ph.HE]].

[58] K. Murase and J. F. Beacom, JCAP 1210, 043 (2012) [arXiv:1206.2595 [hep-ph]].

[59] C. -A. Faucher-Giguere and A. Loeb, JCAP 1001, 005 (2010) [arXiv:0904.3102 [astro-ph.HE]].

[60] A. A. Abdo et al. [Fermi-LAT Collaboration], Science, 325848 (2009); D. Malyshev, I. Cholis, J. D. Gelfand, [arXiv:1002.0587 [astro-ph.HE]].

[61] https://confluence.slac.stanford.edu/display/GLAMCOG/Public+List+ of+LAT-Detected+Gamma-Ray+Pulsars; see also A. A. Abdo et al. [Fermi-LAT Collaboration], Astrophys. J. Suppl. 187, 460-494 (2010) [arXiv:0910.1608 [astro-ph.HE]].

[62] D. Hooper, I. Cholis, T. Linden, J. Siegal-Gaskins and T. Slatyer, Phys. Rev. D 88, 083009 (2013) [arXiv:1305.0830 [astro-ph.HE]].

[63] R. N. Manchester, G. B. Hobbs, A. Teoh and M. Hobbs, Astrophys. J. 129, 1993 (2005); http://www .atnf.csiro.au/people/pulsar/psrcat/.

[64] J. M. Siegal-Gaskins, R. Reesman, V. Pavlidou, S. Profumo and T. P. Walker, Mon. Not. Roy. Astron. Soc. 415, 1074S (2011) [arXiv:1011.5501 [astro-ph.HE]].

[65] F. Zandanel and S. 'i. Ando, arXiv:1312.1493 [astro-ph.HE]. 
[66] T. Arlen et al. [Veritas Collaboration], Astrophys. J. 757, 123 (2012) [arXiv:1208.0676 [astro-ph.HE]].

[67] The Fermi-LAT Collaboration, arXiv:1308.5654 [astro-ph.HE].

[68] M. Ackermann, et al., Astrophys. J. 717, L71 (2010).

[69] D. A. Prokhorov and E. M. Churazov, arXiv:1309.0197 [astro-ph.HE].

[70] B. Huber, C. Tchernin, D. Eckert, C. Farnier, A. Manalaysay, U. Straumann and R. Walter, arXiv:1308.6278 [astro-ph.HE].

[71] R. Feldmann, D. Hooper and N. Y. Gnedin, Astrophys. J. 763, 21 (2013) [arXiv:1205.0249 [astro-ph.HE]].

[72] I. V. Moskalenko and T. A. Porter, Astrophys. J. 692, 54 (2009) [arXiv:0901.0304 [astro-ph.HE]].

[73] S. 'i. Ando and E. Komatsu, Phys. Rev. D 87, 123539 (2013) [arXiv:1301.5901 [astro-ph.CO]].

[74] M. Cirelli, G. Corcella, A. Hektor, G. Hutsi, M. Kadastik, P. Panci, M. Raidal and F. Sala et al., JCAP 1103, 051 (2011) [Erratum-ibid. 1210, E01 (2012)] [arXiv:1012.4515 [hep-ph], arXiv:1012.4515 [hep-ph]].

[75] S. Murray, C. Power and A. Robotham, arXiv:1306.6721 [astro-ph.CO].

[76] J. L. Tinker, A. V. Kravtsov, A. Klypin, K. Abazajian, M. S. Warren, G. Yepes, S. Gottlober and D. E. Holz, Astrophys. J. 688, 709 (2008) [arXiv:0803.2706 [astro$\mathrm{ph}]$.

[77] J. F. Navarro, C. S. Frenk and S. D. M. White, Astrophys. J. 462, 563 (1996) [astro-ph/9508025]. 
[78] J. F. Navarro, C. S. Frenk and S. D. M. White, Astrophys. J. 490, 493 (1997) [astro-ph/9611107].

[79] J. C. Munoz-Cuartas, A. V. Maccio, S. Gottlober and A. A. Dutton, arXiv:1007.0438 [astro-ph.CO].

[80] G. L. Bryan and M. L. Norman, Astrophys. J. 495, 80 (1998) [astro-ph/9710107].

[81] W. Hu and A. V. Kravtsov, Astrophys. J. 584, 702 (2003) [astro-ph/0203169].

[82] L. Gao, C. S. Frenk, A. Jenkins, V. Springel and S. D. M. White, Mon. Not. Roy. Astron. Soc. 419, 1721 (2012) [arXiv:1107.1916 [astro-ph.CO]].

[83] Shin'ichiro Ando, private communication.

[84] V. Springel, J. Wang, M. Vogelsberger, A. Ludlow, A. Jenkins, A. Helmi, J. F. Navarro and C. S. Frenk et al., Mon. Not. Roy. Astron. Soc. 391, 1685 (2008) [arXiv:0809.0898 [astro-ph]].

[85] S. Profumo, K. Sigurdson and M. Kamionkowski, Phys. Rev. Lett. 97, 031301 (2006) [astro-ph/0603373].

[86] J. M. Cornell, S. Profumo and W. Shepherd, Phys. Rev. D 88, 015027 (2013) [arXiv:1305.4676 [hep-ph]].

[87] D. Anderhalden and J. Diemand, JCAP 1304, 009 (2013) [Erratum-ibid. 1308, E02 (2013)] [arXiv:1302.0003 [astro-ph.CO]].

[88] J. Zavala and N. Afshordi, arXiv:1311.3296 [astro-ph.CO].

[89] K. C. Y. Ng, R. Laha, S. Campbell, S. Horiuchi, B. Dasgupta, K. Murase and J. F. Beacom, arXiv:1310.1915 [astro-ph.CO].

[90] A. Klypin, H. Zhao and R. S. Somerville, Astrophys. J. 573, 597 (2002) [astro$\mathrm{ph} / 0110390]$. 
[91] F. Iocco, M. Pato, G. Bertone and P. Jetzer, JCAP 1111, 029 (2011) [arXiv:1107.5810 [astro-ph.GA]].

[92] J. Bovy and S. Tremaine, Astrophys. J. 756, 89 (2012) [arXiv:1205.4033 [astroph.GA]].

[93] R. Catena and P. Ullio, JCAP 1008, 004 (2010) [arXiv:0907.0018 [astro-ph.CO]].

[94] P. Salucci, F. Nesti, G. Gentile and C. F. Martins, Astron. Astrophys. 523, A83 (2010) [arXiv:1003.3101 [astro-ph.GA]].

[95] M. Kuhlen, J. Diemand and P. Madau, Astrophys. J. 686, 262 (2008) [arXiv:0805.4416 [astro-ph]].

[96] S. 'i. Ando, Phys. Rev. D 80, 023520 (2009) [arXiv:0903.4685 [astro-ph.CO]].

[97] K. N. Abazajian, S. Blanchet and J. P. Harding, Phys. Rev. D 85, 043509 (2012) [arXiv:1011.5090 [hep-ph]].

[98] T. Bringmann, F. Calore, M. Di Mauro and F. Donato, arXiv:1303.3284 [astroph.CO].

[99] M. Fornasa, J. Zavala, M. A. Sanchez-Conde, J. M. Siegal-Gaskins, T. Delahaye, F. Prada, M. Vogelsberger and F. Zandanel et al., MNRAS, 429, 1529 (2013) [arXiv:1207.0502 [astro-ph.HE]].

[100] M. Cirelli and G. Giesen, JCAP 1304, 015 (2013) [arXiv:1301.7079 [hep-ph]].

[101] M. Tavakoli, I. Cholis, C. Evoli and P. Ullio, arXiv:1308.4135 [astro-ph.HE].

[102] F. Donato, N. Fornengo and D. Maurin, Phys. Rev. D 78, 043506 (2008) [arXiv:0803.2640 [hep-ph]].

[103] C. Hailey, T. Aramaki, P. von Doetinchem and R. A. Ong, arXiv:1305.0234 [astro-ph.CO]. 
[104] N. Fornengo, L. Maccione and A. Vittino, JCAP 1309, 031 (2013) [arXiv:1306.4171 [hep-ph], arXiv:1306.4171].

[105] C. Evoli, I. Cholis, D. Grasso, L. Maccione and P. Ullio, Phys. Rev. D 85, 123511 (2012) [arXiv:1108.0664 [astro-ph.HE]].

[106] L. Bergstrom, T. Bringmann, I. Cholis, D. Hooper and C. Weniger, Phys. Rev. Lett. 111, 171101 (2013) [arXiv:1306.3983 [astro-ph.HE]]. 


\section{CHAPTER VIII}

\section{Inspecting the Higgs for New Weakly Interacting Particles}

We explore new physics scenarios which are optimally probed through precision Higgs measurements rather than direct collider searches. Such theories consist of additional electroweak charged or singlet states which couple directly to or mix with the Higgs boson; particles of this kind may be weakly constrained by direct limits due to their meager production rates and soft decay products. We present a simplified framework which characterizes the effects of these states on Higgs physics by way of tree level mixing (with neutral scalars) and loop level modifications (from electrically charged states), all expressed in terms of three mixing angles and three loop parameters, respectively. The theory parameters are constrained and in some cases even fixed by ratios of Higgs production and decay rates. Our setup is simpler than a general effective operator analysis, in that we discard parameters irrelevant to Higgs observables while retaining complex correlations among measurements that arise due to the

Written in collaboration with Clifford Cheung and K. M. Zurek. Originally published as Inspecting the Higgs for New Weakly Interacting Particles, JHEP 1304, 074 (2013). arXiv:1302.0314 [hep-ph], MCTP-13-01. 
underlying mixing and radiative effects. We show that certain correlated observations are forbidden, e.g. a depleted ratio of Higgs production from gluon fusion versus vector boson fusion together with a depleted ratio of Higgs decays to $b \bar{b}$ versus $W W$. Moreover, we study the strong correlation between the Higgs decay rate to $\gamma \gamma$ and $W W$ and how it can be violated in the presence of additional electrically charged particles. Our formalism maps straightforwardly onto a variety of new physics models, such as the NMSSM. We show, for example, that with a Higgsino of mass $m_{\chi_{1}^{ \pm}} \gtrsim 100 \mathrm{GeV}$ and a singlet-Higgs coupling of $\lambda=0.7$, the photon signal strength can deviate from the vector signal strength by up to $\sim 40-60 \%$ while depleting the vector signal strength by only $5-15 \%$ relative to the Standard Model.

\subsection{Introduction}

The ATLAS [1] and CMS [2] collaborations have presented definitive evidence for the existence of a new, Higgs-like boson with a mass of order $125 \mathrm{GeV}$. At present, observations are broadly consistent with Standard Model (SM) expectations [3], par-

ticularly in the $W W[4], Z Z[5]$, and $b \bar{b}$ [6] decay channels, a modest surplus in the $\gamma \gamma[7]$ channel notwithstanding.

Meanwhile, physics beyond the SM has yet to appear in dedicated searches conducted at the LHC. Searches for supersymmetry (SUSY), extra dimensions, technicolor, and other models have all turned up empty-handed, suggesting the possibility that precision Higgs measurements might offer our best handle on new physics in the coming years. While present observations carry large error bars, experimental precision will improve over time, providing more definitive constraints on deviations from a SM Higgs sector.

What manner of new physics would appear first in precision Higgs physics rather 
than in direct searches? Such particles are unlikely to be colored, since strongly interacting particles are produced en masse and typically subject to stringent searches involving jets. Hence, the new states should carry electroweak charges alone - a scenario notoriously difficult to disentangle, even when the new states are relatively light. In the context of SUSY, for example, light charginos or staus can escape detection without the aid of strongly produced squarks or gluinos. If the new particles couple directly to or mix with the Higgs, however, then precision Higgs measurements may offer our leading experimental probe.

There are myriad theoretical motivations for new particles which couple directly to the Higgs boson. Indeed, such states are required to regulate the quadratic divergences of the Higgs in any model that addresses the gauge hierarchy problem. In many cases, these interactions can substantially modify Higgs boson physics [8-46], especially in processes like $h \rightarrow \gamma \gamma$. On their own, however, new electroweak charged states offer diminishing returns for modifying observables like the diphoton branching fraction, except in extreme regions of parameter space with very large couplings $[8,47-49]$.

On the other hand, the presence of additional scalars in the Higgs sector can change this picture dramatically because mixing between the scalars introduces important tree level effects. If new particles mix with the Higgs boson, then these states are scalars in the singlet, doublet, triplet, etc. representations, and will in general acquire vacuum expectation values $(\mathrm{VEVs})$. Triplet VEVs are disfavored by precision electroweak measurements, while higher order representations are somewhat contrived. Thus, the most natural case of study is a Higgs sector comprised of mixed singlets and doublets.

This sequence of logic leads us to the effective theory which is the focus of the present work: the SM augmented by a scalar singlet and doublet which mix with the Higgs boson, all of which can couple to additional new states of arbitrary electroweak 
charges. The purpose of this paper is to describe and quantify the phenomenology of this setup in a general, relatively model independent fashion. Our central conclusions are as follows:

- Three mixing angles and three loop parameters are sufficient to characterize the span of observable effects on Higgs properties in this wide class of theories. This framework is substantially simpler than a canonical effective Lagrangian approach, which entails new particles and theory parameters which play no role for precision Higgs physics.

- Experimental observables such as Higgs production and decay rates can be directly "inverted" to determine the theory parameters of this setup. Concretely, one can employ Fig. 8.1 to ascertain two mixing angles, Fig. 8.2 for the third mixing angle, and Fig. 8.4 for information about the loop parameters. Critical to this determination are the ratio of gluon fusion and vector boson fusion production, and the Higgs decay rates to photons and bottom quarks relative to massive electroweak gauge bosons.

- Our framework is simpler than an effective operator formalism which parameterizes arbitrary couplings between $h$ to SM fields and, crucially, it preserves important correlations among observables that encode the underlying effects of mixing and loops. For example, Fig. 8.1 shows how certain combinations of observations cannot occur. Likewise, we investigate the tight correlation between the Higgs decay rate to $\gamma \gamma$ and $W W$ and how it can be can broken through important loop effects.

- This framework applies to a broad class of models which include additional scalar singlets and doublets that mix with the Higgs, together with new electrically charged particles. We discuss our results in the specific context of the NMSSM. 
The outline of this paper is as follows. In Sec. 8.2 we define our framework and relate the associated theory parameters to physical quantities. We discuss the effects of tree level mixing in Sec. 8.3 and in the effects of loops in Sec. 8.4. Finally, in Sec. 8.5 we discuss the specific application of our framework to the NMSSM before concluding.

\subsection{Framework}

\subsubsection{Theory Parameterization}

Our theoretical framework assumes that the observed Higgs boson, $h$, is an admixture of the neutral components of two Higgs doublets, $\phi_{u}$ and $\phi_{d}$, and a singlet, $\phi_{s}$, which acquire VEVs such that

$$
\phi_{I}=v_{I}+H_{I}, \quad I=u, d, s
$$

where $v_{u}^{2}+v_{d}^{2}=v^{2} \simeq(246 \mathrm{GeV})^{2}$, while $v_{u} / v_{d}=\tan \beta$ and $v_{s}$ are free parameters. We define $h$ to be the lightest mass eigenstate, which is a linear combination of the field fluctuations

$$
\begin{aligned}
h & =\sum_{I} P_{I} H_{I} \\
P_{I} & =(\cos \alpha \cos \gamma,-\sin \alpha \cos \gamma,-\sin \gamma),
\end{aligned}
$$

where $P_{I}$ is, by construction, an orthonormal vector that defines a column of the scalar mixing matrix. Here $\alpha$ characterizes the mixing between $\phi_{u}$ and $\phi_{d}$, while $\gamma$ parameterizes the amount of mixing into $\phi_{s}$. A priori, $\alpha$ and $\gamma$ label arbitrary angles in spherical coordinates, so $\alpha$ and $\gamma$ are periodic over domains of size $2 \pi$ and $\pi$, respectively. As we will see later on, many physical observables will depend on these angles with a higher frequency of periodicity. For later convenience, we also define a 
difference angle

$$
\delta=\alpha-\beta+\pi / 2,
$$

which measures deviations from the SM "decoupling" limit, $\delta=0$.

In the SM, it is well known that the couplings of $h$ to other fields are fixed by low-energy Higgs theorems [50]. In particular, starting with the SM action below the electroweak symmetry breaking scale, the leading Higgs couplings are obtained by sending $v \rightarrow v+h$, so that all $h$ couplings go like $\partial / \partial v$. In our framework, this generalizes to the statement that $h$ couples proportionally to $\sum_{I} P_{I} \partial / \partial v_{I}$. For any particles that derive mass from electroweak symmetry breaking, it is convenient to define the dimensionless quantities

$$
\begin{aligned}
d_{i} & =\sum_{I} P_{I} \eta_{I, i} \\
\eta_{I, i} & =\frac{v}{m_{i}} \frac{\partial m_{i}}{\partial v_{I}}
\end{aligned}
$$

where $i$ labels the massive electroweak gauge bosons or fermions and $m_{i}$ denotes the mass of particle $i$. Note that $d_{i}=1$ in the SM limit because the quarks, leptons and massive electroweak gauge bosons all acquire tree level masses from electroweak symmetry breaking alone. Meanwhile, since the photon and gluon do not acquire mass from electroweak symmetry breaking, Eq. (8.5) does not apply to them. We will define these radiatively induced couplings shortly.

For the massive electroweak gauge bosons and up- and down-type quarks, we have

$$
\begin{aligned}
d_{V}=\cos \gamma \sin (\beta-\alpha) & =\cos \gamma \cos \delta \\
d_{t}=\cos \gamma \cos \alpha / \sin \beta & =\cos \gamma \cos \delta(1+\tan \delta \cot \beta) \\
d_{b}=-\cos \gamma \sin \alpha / \cos \beta & =\cos \gamma \cos \delta(1-\tan \delta \tan \beta),
\end{aligned}
$$


where in our setup we assume that $\phi_{u}$ couples to up-type quarks, $\phi_{d}$ couples to downtype quarks and leptons, and $\phi_{s}$ carries no renormalizable couplings directly to quarks, leptons, or SM gauge bosons. As is well-known, this restriction on $\phi_{u}$ and $\phi_{d}$ couplings provides a convenient way for evading stringent constrains on flavor changing neutral currents. Such a choice can be straightforwardly enforced by discrete symmetries or holomorphy, in the case of SUSY.

Our framework also accounts for the possibility that the $\phi_{I}$ can couple to additional particles beyond the SM. When $i$ labels such a new state, we have

$$
\begin{aligned}
d_{i} & =\cos \gamma\left(\cos \alpha \eta_{u, i}-\sin \alpha \eta_{d, i}\right)-\sin \gamma \eta_{s, i} \\
& =\cos \gamma\left(\sin (\beta+\delta) \eta_{u, i}+\cos (\beta+\delta) \eta_{d, i}\right)-\sin \gamma \eta_{s, i}
\end{aligned}
$$

where $\eta_{I, i}$ is taken to be an unknown loop parameter that will be constrained by experiment. Because $i$ labels a new particle, $d_{i}$ has no counterpart in the SM, but it can be can be straightforwardly extracted from a given ultraviolet model using Eqs. (8.5) and (8.6). Because $\eta_{I, i}$ characterizes the power of $v_{I}$ with which $m_{i}$ scales we expect $\left|\eta_{I, i}\right|=1$ in renormalizable theories in which the entirety of $m_{i}$ derives from electroweak symmetry breaking [51]. Absent fine-tuning between tree level mass contributions and electroweak symmetry breaking contributions to $m_{i}$, the naive expectation is that in general $\left|\eta_{I, i}\right| \lesssim 1$.

For couplings that only arise at loop level, $v_{I}$ dependence enters the action through particle mass thresholds that influence the running couplings of electromagnetism and the strong interactions. As in the SM, these effects are what induce the couplings of $h$ to photons and gluons. For these interactions we define

$$
\begin{aligned}
& d_{\gamma}=\sum_{i} \mathcal{A}_{\gamma, i} d_{i} \\
& d_{g}=\sum_{i} \mathcal{A}_{g, i} d_{i},
\end{aligned}
$$


where $i$ sums over all particles which acquire mass from electroweak symmetry breaking, including new states. The dimensionless constants $\mathcal{A}_{\gamma, i}$ and $\mathcal{A}_{g, i}$ are defined as

$$
\begin{aligned}
& \mathcal{A}_{\gamma, i}=A_{J(i)}\left(\tau_{i}\right) C_{\gamma, i} /\left(\sum_{j \in \mathrm{SM}} A_{J(j)}\left(\tau_{j}\right) C_{\gamma, j}\right) \\
& \mathcal{A}_{g, i}=A_{J(i)}\left(\tau_{i}\right) C_{g, i} /\left(\sum_{j \in \mathrm{SM}} A_{J(j)}\left(\tau_{j}\right) C_{g, j}\right),
\end{aligned}
$$

and characterize the relative importance of loop corrections from each particle. Note that $\sum_{i \in \mathrm{SM}} \mathcal{A}_{\gamma, i}=\sum_{i \in \mathrm{SM}} \mathcal{A}_{g, i}=1$, which enforces that $d_{\gamma}=d_{g}=1$ in the SM limit. Here $A_{J(i)}\left(\tau_{i}\right)$ are kinematic functions of the spin $(J(i)=0,1 / 2,1)$ and the mass $\left(\tau_{i}=m_{h}^{2} / 4 m_{i}^{2}\right)$ of the particle $i$ in the loop. The functions $A_{J(i)}\left(\tau_{i}\right)$ asymptote to beta function coefficients $b_{0}=1 / 3, b_{1 / 2}=4 / 3, b_{1}=-7$ in the $\tau_{i} \rightarrow 0$ limit of infinitely heavy mass of the loop particle; full expressions of the $A_{J(i)}\left(\tau_{i}\right)$ are given in, e.g. [45]. Here we have defined $C_{\gamma, i}=N_{c, i} Q_{i}^{2}$, for $N_{c, i}$ colors and charge $Q_{i}$, and $C_{g, i}=\frac{3}{2} C_{2}\left(r_{i}\right)$, for a quadratic Casimir $C_{2}$ of the color representation $r_{i}$ [32]. Because the Higgs coupling to photons is dominated by the $W$ boson loop (with subdominant and destructively interfering contributions from top and bottom quark loops) and the Higgs coupling to gluons is dominated by top and bottom quarks, the relevant SM contributions to Eq. (8.9) and Eq. (8.10) come from

$$
\begin{aligned}
& \mathcal{A}_{\gamma, V} \simeq 1.277-0.006 i \\
& \mathcal{A}_{\gamma, t} \simeq-0.281+0.001 i \\
& \mathcal{A}_{\gamma, b} \simeq 0.004+0.005 i \\
& \mathcal{A}_{g, t} \simeq 1.050+0.077 i \\
& \mathcal{A}_{g, b} \simeq-0.050-0.077 i
\end{aligned}
$$

assuming $m_{h}=125.5 \mathrm{GeV}$. Note that while the $b$ contributions are naively negligible, 
they grow like $\tan ^{2} \beta$ relative to the $t$ contributions and must be included.

\subsubsection{Observables Parameterization}

Next, let us consider the dependence of physical quantities on these theory parameters. To do so, we define

$$
R[\mathcal{O}]=\mathcal{O} / \mathcal{O}_{\mathrm{SM}}
$$

to be the ratio of a given observable $\mathcal{O}$ to its $\mathrm{SM}$ value, $\mathcal{O}_{\mathrm{SM}}$. In this notation, we find that the following important partial width ratios go as

$$
\begin{aligned}
R[\Gamma(h \rightarrow V V)] & =\left|d_{V}\right|^{2} \\
R[\Gamma(h \rightarrow b b)] & =\left|d_{b}\right|^{2} \\
R[\Gamma(h \rightarrow \gamma \gamma)] & =\left|d_{\gamma}\right|^{2} .
\end{aligned}
$$

Note that $R[\Gamma(h \rightarrow \ell \ell)]=R[\Gamma(h \rightarrow b b)]$ in our framework because $\phi_{d}$ provides the masses for both the down-type quarks and the leptons.

Important production cross-section ratios go as

$$
\begin{aligned}
R[\sigma(g g \rightarrow h)] & =\left|d_{g}\right|^{2} \quad \text { (gluon fusion) } \\
R[\sigma(V V \rightarrow h)] & =\left|d_{V}\right|^{2} \quad \text { (vector boson fusion) }
\end{aligned}
$$

The cross-section ratio for $V h$ associated production scales the same as for vector boson fusion $(\mathrm{VBF})$, since both processes involve the Higgs coupling to the massive electroweak gauge bosons. As noted earlier, our analysis will not include new strongly interacting particles because such states are likely to be observed first in direct collider searches rather than precision Higgs physics. Furthermore, such states tend to drive a separation of gluon fusion and vector boson fusion production that is not observed 
in the data [3]. In addition, if one is interested in driving enhancements to $h \rightarrow \gamma \gamma$, many models require large scalar mixing (through $A$ terms) [15], which can in turn induce vacuum instability [48]. As a result, the dominant contribution to gluon fusion arises from top and bottom quark loops, so

$$
\left|d_{g}\right|^{2} \simeq\left|\mathcal{A}_{g, t} d_{t}+\mathcal{A}_{g, b} d_{b}\right|^{2}
$$

From Eq. (8.13) we see that the $d_{t}$ contribution is weighted more heavily than $d_{b}$. However, as noted earlier, this can be compensated by important $\tan \beta$ effects. Note that Eqs. (8.18) through (8.20) imply that all relevant production modes go as $R[\sigma(j j \rightarrow h)] \propto \cos ^{2} \gamma$

It will be convenient to present our results in terms of signal strength modifiers which are employed by experimentalists. For the process $j j \rightarrow h \rightarrow i i$, we have

$$
\begin{aligned}
R_{i}^{j} & \equiv R[\sigma(j j \rightarrow h) \times \operatorname{Br}(h \rightarrow i i)] \\
& =R\left[\sigma(j j \rightarrow h) / \Gamma_{\text {tot }}\right] R[\Gamma(h \rightarrow i i)] \\
& =\widehat{R}^{j}\left|d_{i}\right|^{2},
\end{aligned}
$$

where $\widehat{R}^{j} \equiv R\left[\sigma(j j \rightarrow h) / \Gamma_{\text {tot }}\right]$ is defined as the ratio of the production cross-section ratio to the total width ratio and $\Gamma_{\text {tot }}$ is the full width, which varies like

$$
R\left[\Gamma_{\mathrm{tot}}\right]=\sum_{i} \operatorname{Br}(h \rightarrow i i)\left|d_{i}\right|^{2}
$$

Here $\operatorname{Br}(h \rightarrow i i)$ denotes the SM Higgs branching fraction of $h \rightarrow i i$, where $i$ runs over all kinematically accessible final states. For our analysis, we use the branching fractions for $m_{h}=125.5 \mathrm{GeV}$ shown in Table 8.1. In principle, there can exist additional particles beyond the SM to which the Higgs can decay. Throughout the present analysis, however, we neglect the possibility of such new light states below 
the Higgs mass threshold, so in Eq. (8.24) $i$ labels SM particles alone.

\begin{tabular}{|c||c|c|c|c|c|c|c|}
\hline$i$ & $b$ & $W$ & $g$ & $\tau$ & $c$ & $Z$ & $\gamma$ \\
\hline $\operatorname{Br}(h \rightarrow i i)$ & $56.9 \%$ & $22.3 \%$ & $8.52 \%$ & $6.24 \%$ & $2.87 \%$ & $2.76 \%$ & $0.228 \%$ \\
\hline
\end{tabular}

Table 8.1: Branching fractions for $h \rightarrow i i$ for $m_{h}=125.5 \mathrm{GeV}$ from [52].

Because the SM branching ratios are dominated by tree level decays to massive particles that exclusively couple through the doublet Higgses $\phi_{u}$ and $\phi_{d}$, we expect that $R\left[\Gamma_{\text {tot }}\right] \propto \cos ^{2} \gamma$ as long as $\gamma$ is not very large. However, if $\gamma \simeq \mathcal{O}( \pm \pi / 2)$ the decays will be dominated by decays through the singlet component $\phi_{s}$ and we instead find $R\left[\Gamma_{\text {tot }}\right] \propto \sin ^{2} \gamma$. As argued above, $R[\sigma(j j \rightarrow h)] \propto \cos ^{2} \gamma$ holds for all

production channels of interest so $\widehat{R}^{j}$ is independent of $\gamma$ for most mixing angles, but as $\gamma \rightarrow \pm \pi / 2$ the loop level contribution to the width from $\phi_{s}$ begins to dominate the full width, in which case $\widehat{R}^{j} \propto \cot ^{2} \gamma \rightarrow 0$.

\subsection{Tree Level Effects}

In this section we consider the effect on Higgs properties from tree level modifications to the SM scalar sector. It will be particularly convenient to consider certain ratios of quantities in order to reduce systematics, including

$$
\frac{R_{b}^{j}}{R_{V}^{j}}=\frac{R_{\ell}^{j}}{R_{V}^{j}}=\frac{\left|d_{b}\right|^{2}}{\left|d_{V}\right|^{2}}=(1-\tan \delta \tan \beta)^{2},
$$

where the production mode $j$ is arbitrary, e.g. gluon fusion or vector boson fusion. We also consider the ratio

$$
\frac{R_{i}^{g}}{R_{i}^{V}} \simeq \frac{\left|\mathcal{A}_{g, t} d_{t}+\mathcal{A}_{g, b} d_{b}\right|^{2}}{\left|d_{V}\right|^{2}}=\left|\mathcal{A}_{g, t}(1+\tan \delta \cot \beta)+\mathcal{A}_{g, b}(1-\tan \delta \tan \beta)\right|^{2}
$$

where the decay mode $i$ is arbitrary. Interestingly, Eq. (8.25) and Eq. (8.26) provide two equations for two unknowns, $\delta$ and $\beta$, which can be solved for in terms of experi- 

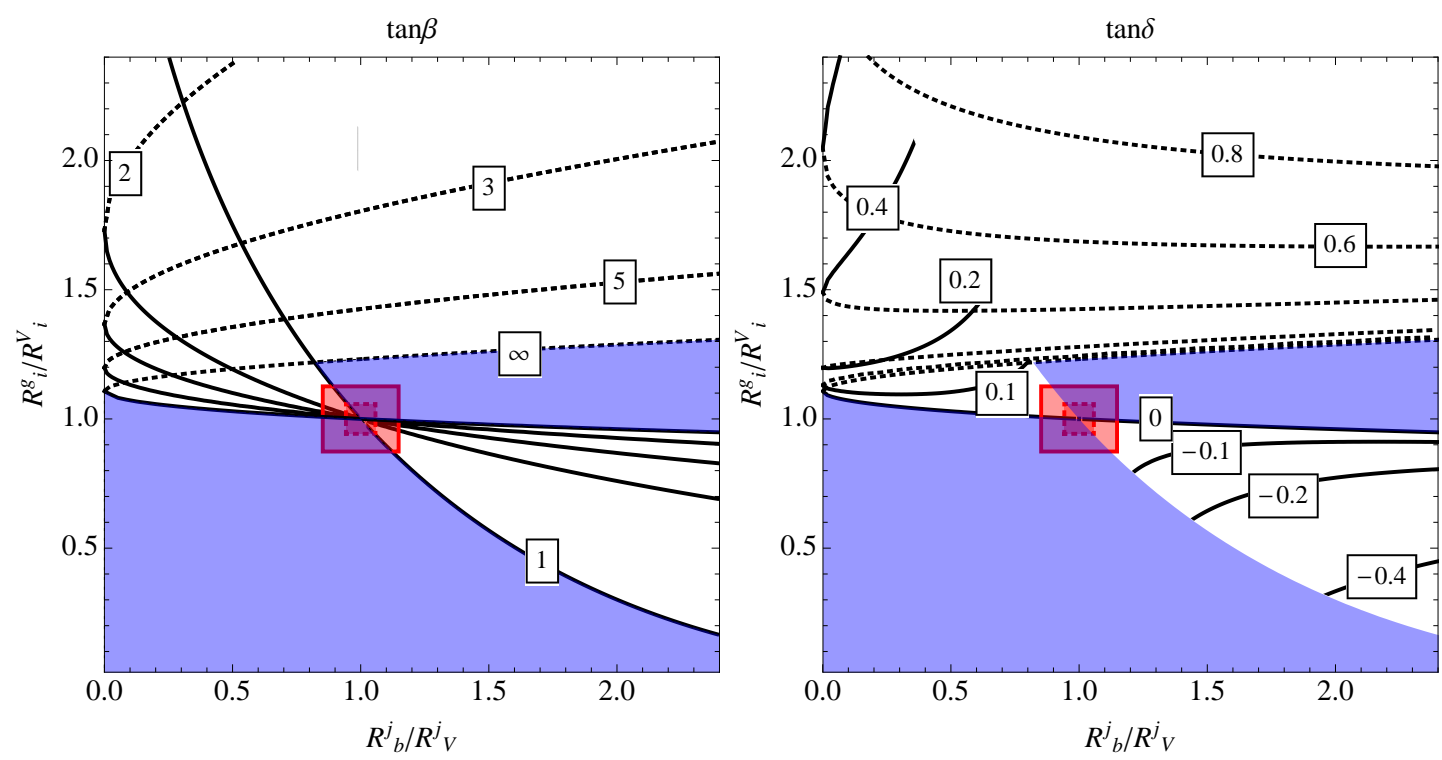

Figure 8.1: Contours of $\tan \beta$ (left) and $\tan \delta$ (right) as functions of $\left(R_{i}^{g} / R_{i}^{V}, R_{b}^{j} / R_{V}^{j}\right)$, which are obtained directly from experiment. The red shaded regions with solid (dashed) borders show values that will remain consistent with the SM with 300 (3000) $\mathrm{fb}^{-1}$ and blue shaded regions show values which cannot be observed within this framework. The solid (dotted) curves show the region where $d_{b} / d_{V}$ is positive (negative).

mental inputs. To facilitate this mapping, we plot $\tan \delta$ and $\tan \beta$ as functions of the signal strength modifiers $R_{b}^{j} / R_{V}^{j}$ and $R_{i}^{g} / R_{i}^{V}$ in Fig. 8.1. The solid (dotted) curves show the region where $d_{b} / d_{V}$ is positive (negative). For models which are relatively SM-like, this quantity should be positive and close to unity; large new physics contributions are required to flip its sign. The shaded red boxes in Fig. 8.1 denote the range of measurements consistent within $1 \sigma$ uncertainty of the SM measured at the LHC at $14 \mathrm{TeV}$ energies and 300 and $3000 \mathrm{fb}^{-1}$ luminosity. Any theoretical models enclosed by this region will be very difficult to distinguish from a SM hypothesis. Outside of the red region, however, Fig. 8.1 can be straightforwardly used to extract the mixing angles. We list the relative errors that will be compatible with the SM in Tab. 8.2, combining in quadrature and making the identification $d_{Z}=d_{V}$ from the values given in Table 2.3 of [53].

The blue regions in Fig. 8.1 indicate the parameter space of observables which 


\begin{tabular}{|c||c|c|c|}
\hline ratio & $R_{i}^{g} / R_{i}^{V}$ & $R_{b}^{j} / R_{V}^{j}$ & $R_{\gamma}^{j} / R_{V}^{j}$ \\
\hline uncertainty & $12.6 \%(5.76 \%)$ & $14.8 \%(5.76 \%)$ & $11.5 \%(3.61 \%)$ \\
\hline
\end{tabular}

Table 8.2: Projected uncertainties on ratios of signal strength modifiers for the 14 TeV LHC with $300(3000) \mathrm{fb}^{-1}$ of data.

cannot be observed within this framework. The forbidden regions in the upper right and lower right quadrants cannot occur because $\tan \beta$ falls outside of the allowed range $\tan \beta>1$. Far outside of this range, the top quark Yukawa coupling becomes non-perturbative. Thus, a general prediction is that $R_{b}^{j} / R_{V}^{j}$ and $R_{i}^{g} / R_{i}^{V}$ should not be observed deep within these blue regions. Furthermore, that the lower left quadrant of Fig. 8.1 is disallowed can be easily understood as follows. In order to decrease $R_{b}^{j} / R_{V}^{j}$ and $R_{i}^{g} / R_{i}^{V}$ simultaneously, one requires a suppression of the couplings of the Higgs to both the top and bottom quarks relative to the vector bosons. However, because we consider theories in which $\phi_{u}$ and $\phi_{d}$ couple exclusively to up-type and down-type quarks, respectively, the coupling of the Higgs to top and bottom quarks are necessarily anti-correlated. Similar logic would imply that entirety of the upper right quadrants of Fig. 8.1 should also be forbidden. This is true if $d_{b}>0$; however, if new physics contributions are so large as to flip the sign to $d_{b}<0$, then these regions in the upper right quadrants become allowed again.

Fig. 8.2 is a complementary representation of the same information as in Fig. 8.1, except that $R_{b}^{j} / R_{V}^{j}$ and $R_{i}^{g} / R_{i}^{V}$ are shown as functions of $\tan \delta$ and $\tan \beta$. One can see that $\tan \delta$ has support in two distinct bands in each plot of Fig. 8.2, but the allowed value is constrained by matching both measurements, as corroborated by Fig. 8.1. One also notices that $R_{b}^{j} / R_{V}^{j}$ and $R_{i}^{g} / R_{i}^{V}$ are quite sensitive to $\delta$, but not so dependent on $\beta$. For this reason, a precise measurement of $\beta$ is more difficult than a determination of $\delta$, as confirmed by the contours in Figs. 8.1 and 8.2. This is because $\tan \beta$ cannot be extracted in the decoupling limit, so any constraint on $\tan \beta$ necessarily requires an observed deviation in SM-like behavior through $\tan \delta$ first. 

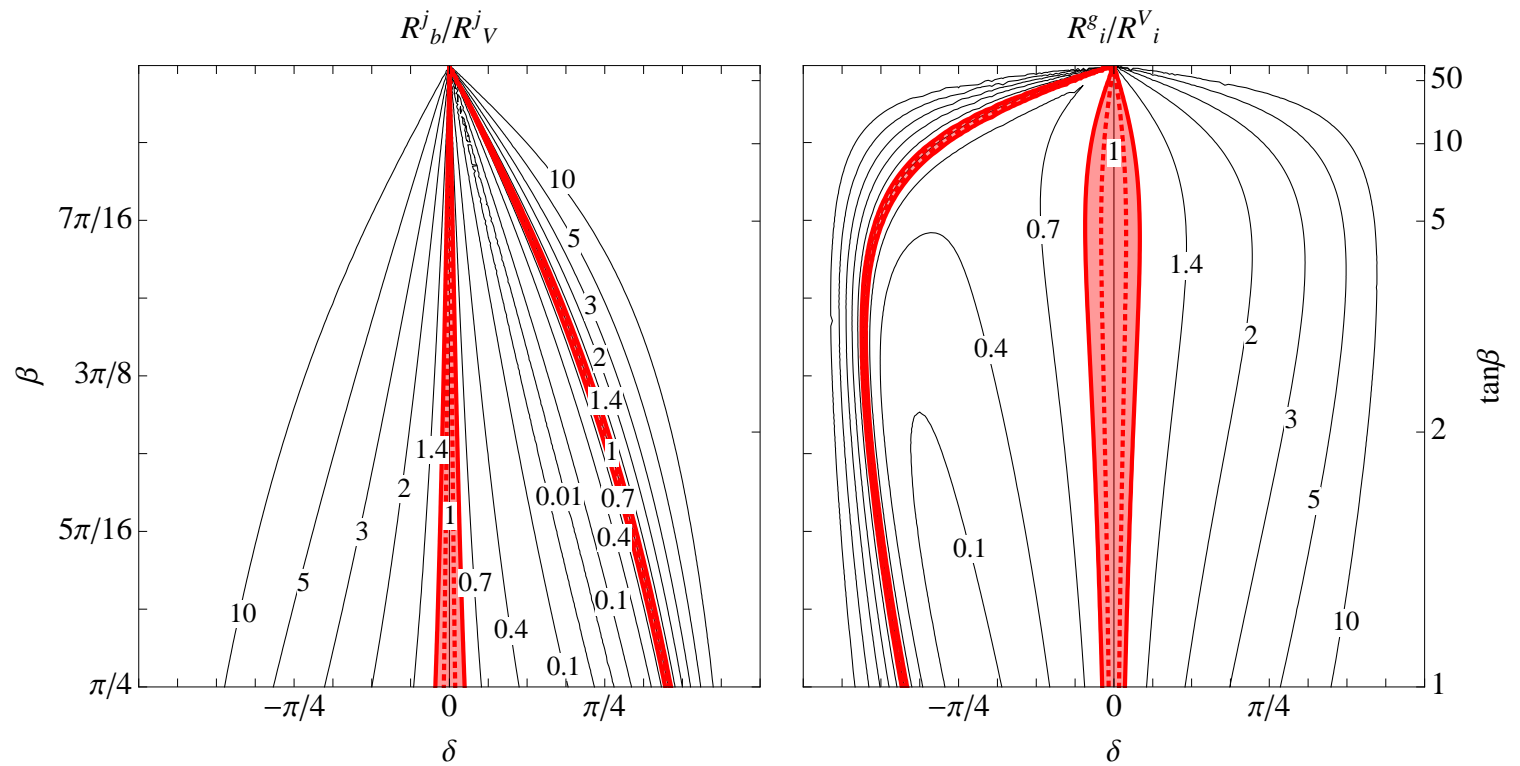

Figure 8.2: Contours of $R_{b}^{j} / R_{V}^{j}$ (left) and $R_{i}^{g} / R_{i}^{V}$ (right) as a function of theory parameters $(\delta, \beta)$. The red shaded regions are as in Fig. 8.1.

Once $\delta$ and $\beta$ are determined from data, they can be used to extract other theory parameters from Higgs measurements. Concretely, given values of $\delta$ and $\beta$, the quantity $\cos ^{2} \gamma$ can be inferred from a number of different observables, including $R_{V}^{g}$, $R_{V}^{V}, R_{b}^{g}$, and $R_{b}^{t}$ (where the $t$ superscript denotes top quark associated Higgs production). Crucially, to a very good approximation these quantities all carry the same $\cos ^{2} \gamma$ dependence, at least when $\gamma \neq \pm \pi / 2$. Away from that limit of large $\gamma$ we have $\left.R_{i}^{j}\right|_{\gamma=0} \simeq R_{i}^{j} / \cos ^{2} \gamma$, which we plot as a function of $\beta$ and $\delta$ in Fig. 8.3. If $\beta$ and $\delta$ have been extracted from observables, and $R_{i}^{j}$ has been measured, then $\gamma$ can be extracted from Fig. 8.3.

We now explain some of the features of Fig. 8.3, starting with the upper left panel. By going to the anti-decoupling limit, $\delta= \pm \pi / 2$, we can tune the vector coupling arbitrarily low while maintaining a nonzero Higgs width to SM particles. In contrast, it is not possible to increase $R_{V}^{j}$ without bound: there is a maximum around where 

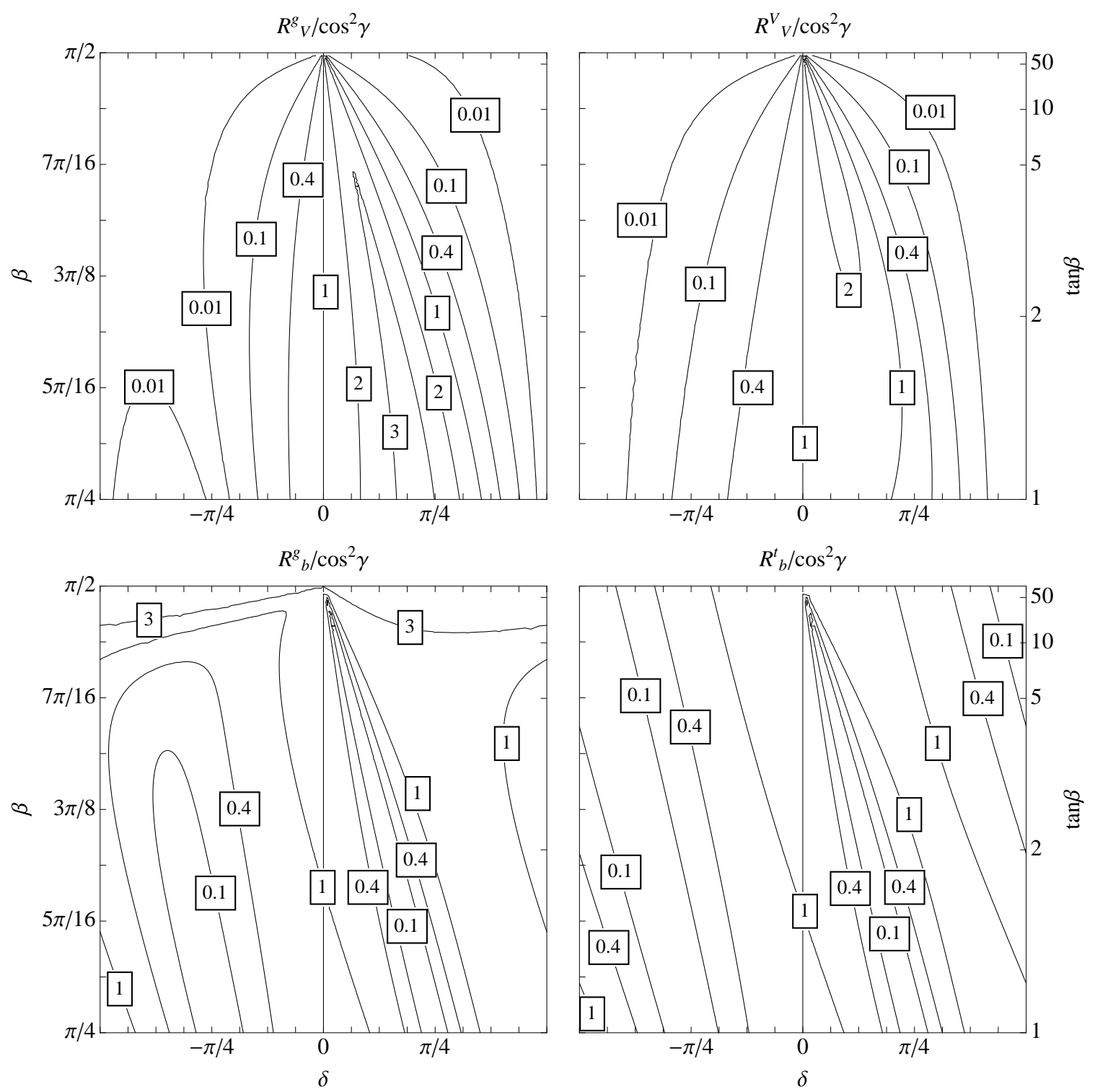

Figure 8.3: Contours of $R_{i}^{j} / \cos ^{2} \gamma$ (which we approximate by $\left.R_{i}^{j}\right|_{\gamma=0}$ ) for $V$ (top row) and $b$ (bottom row) final states from a variety of production channels. Once $\delta$ and $\beta$ are determined from observation, measuring $R_{i}^{j}$ can then be used to obtain $\gamma$.

the $b$ width is zero. Taking $\delta>0(\delta<0)$ corresponds to increasing the amount of $\phi_{u}$ $\left(\phi_{d}\right)$ in the physical Higgs, which boosts the coupling of $h$ to top (bottom) quarks. Thus, for $\delta>0$ the gluon fusion rate $\sigma(g g \rightarrow h)$ is boosted through the top loop, while the full width $\Gamma_{\text {tot }}$ is depleted because of the reduction in the dominant width 
to $b \bar{b}$. These effects conspire to increase $\widehat{R}^{g}$, and at $\tan \beta=1$ this effect outweighs the decrease in $d_{V}$ for $\delta \lesssim \pi / 4$ so that we see an increase for larger $\delta$. Similar effects are seen in the top right panel, which shows the same decay but for vector boson fusion or vector boson associated production, but which is even less sensitive to $\tan \beta$. For the bottom panels, we show $R_{b}^{g}$ and $R_{b}^{t}$ with no singlet mixing, which illustrate that it is very hard to boost bottom production unless we go to very large $\tan \beta$ while simultaneously avoiding the decoupling limit. Bottom production can be increased more effectively by going to large $\tan \beta$ in the case of gluon fusion as compared to top associated production because the gluon loop contains a bottom piece. Thus, these panels are distinct as $\beta \rightarrow \pi / 2$, but they are otherwise very similar.

\subsection{Loop Level Effects}

With results for tree-level processes in hand, we can now consider the multi-faceted effects in the loop-mediated process $h \rightarrow \gamma \gamma$. The mixing angles $\delta, \beta$, and $\gamma$ and three loop parameters $\eta_{I, i}$ enter. We will find again that the physics can be more easily extracted and understood by normalizing to the tree level rate to gauge bosons $R_{V}^{j}$. The diphoton signal strength at leading order is

$$
d_{\gamma} \simeq \mathcal{A}_{\gamma, V} d_{V}+\mathcal{A}_{\gamma, t} d_{t}+\mathcal{A}_{\gamma, b} d_{b}+\sum_{i \notin \mathrm{SM}} \mathcal{A}_{\gamma, i} d_{i}
$$

where we have approximated by only including the dominant SM loop contributions from the electroweak vector bosons and the top and bottom quarks. Dividing both sides of Eq. (8.27) by $d_{V}$ and rearranging terms using the fact that $\mathcal{A}_{\gamma, V}+\mathcal{A}_{\gamma, t}+\mathcal{A}_{\gamma, b}=$ 
1 , we find that

$$
\begin{aligned}
\frac{d_{\gamma}}{d_{V}} & \simeq 1+\mathcal{A}_{\gamma, t}\left(\frac{d_{t}}{d_{V}}-1\right)+\mathcal{A}_{\gamma, b}\left(\frac{d_{b}}{d_{V}}-1\right)+\frac{1}{d_{V}} \sum_{i \notin \mathrm{SM}} \mathcal{A}_{\gamma, i} d_{i} \\
& =1+\epsilon(\beta) \tan \delta+\frac{1}{d_{V}} \sum_{i \notin \mathrm{SM}} \mathcal{A}_{\gamma, i} d_{i},
\end{aligned}
$$

where we have defined a function $\epsilon(\beta)=\mathcal{A}_{\gamma, t} \cot \beta-\mathcal{A}_{\gamma, b} \tan \beta$. Simply squaring Eq. (8.29), we can recast the same information in terms of experimental observables, SO

$$
\frac{R_{\gamma}^{j}}{R_{V}^{j}} \simeq\left|1+\epsilon(\beta) \tan \delta+\frac{1}{d_{V}} \sum_{i \notin \mathrm{SM}} \mathcal{A}_{\gamma, i} d_{i}\right|^{2} .
$$

The first, second and third terms in the above expression correspond to (i) the SM contribution, (ii) the effect of mixing on the $t$ and $b$ Yukawas, and (iii) the effects of any additional charged particles beyond the SM.

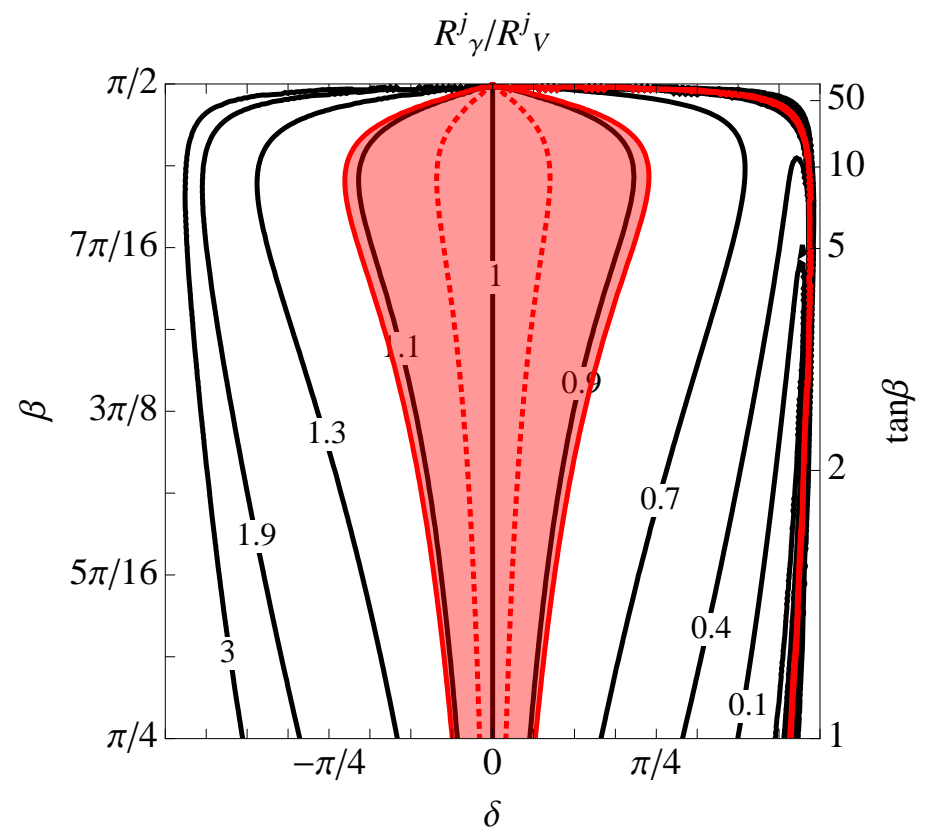

Figure 8.4: Contours of $R_{\gamma}^{j} / R_{V}^{j}$ in the plane of theoretical parameters $(\delta, \beta)$, with no new charged particles beyond the SM present. The red shaded regions are as in Fig. 8.1. 
Let us consider signal strengths in the case with no new charged particles beyond the SM, so that only mixing effects induce deviations from unity. Thus, only the contributions from (i) and (ii) are present in Eq. (8.30),

$$
\frac{R_{\gamma}^{j}}{R_{V}^{j}}=|1+\epsilon(\beta) \tan \delta|^{2} .
$$

In Fig. 8.4 we map out contours of Eq. (8.31). Comparing Figs. 8.2 and 8.4, we see that $R_{V}^{j}$ is more tightly correlated with $R_{\gamma}^{j}$ than with $R_{b}^{j}$, since in the $(\delta, \beta)$ plane, $R_{\gamma}^{j} / R_{V}^{j}$ spans over a much more narrow range than $R_{b}^{j} / R_{V}^{j}$. The reason for this correlation is obvious: $h \rightarrow \gamma \gamma$ is a process dominated by a $W$ loop, so it is highly correlated with the decay $h \rightarrow W W$. Meanwhile, $h \rightarrow b b$ and $h \rightarrow W W$ are uncorrelated because the Higgs coupling to bottom quarks is controlled only by the $\phi_{d}$ component of $h$, while the Higgs coupling to electroweak vector bosons is controlled by both the $\phi_{u}$ and $\phi_{d}$ components of $h$. Thus in order to decouple the Higgs rate to photons relative to the rate to gauge bosons, i.e. to push $R_{\gamma}^{j} / R_{V}^{j}$ far from unity, loop effects from new charged particles must be included. Breaking the correlation between these signal strengths will be one of the primary effects we are investigating, but loop effects will be critical for doing so.

Let us now investigate these loop effects. As expected, in the decoupling limit, $\delta \rightarrow 0$, the effects of (ii) vanish but (iii) can still play an important role. We plot the unknown quantity $\sum_{\mathrm{i} \notin \mathrm{SM}} \mathcal{A}_{\gamma, i} d_{i} / d_{V}$ in Fig. 8.5 as a function of the ratios of signal strengths that appear in Eq. (8.30), taking the low $\tan \beta$ limit such that $d_{g} \simeq d_{t}$, and thus $\epsilon(\beta) \simeq \mathcal{A}_{\gamma, t}\left(d_{g} / d_{V}-1\right)$. Because the signal strengths are related to $d_{\gamma}$ and $d_{g}$ by squaring, the latter are only fixed up to a sign ambiguity. In the left (right) panel we show contours of $\sum_{\mathrm{i} \notin \mathrm{SM}} \mathcal{A}_{\gamma, i} d_{i} / d_{V}$ where the sign of $d_{\gamma} / d_{V}$ is positive (negative). In both panels, the solid (dotted) curves show regions where $d_{g} / d_{V}$ is positive (negative). Note that negative values of $d_{g} / d_{V}$ or $d_{\gamma} / d_{V}$ require large effects from new physics, 
and are far from SM-like.
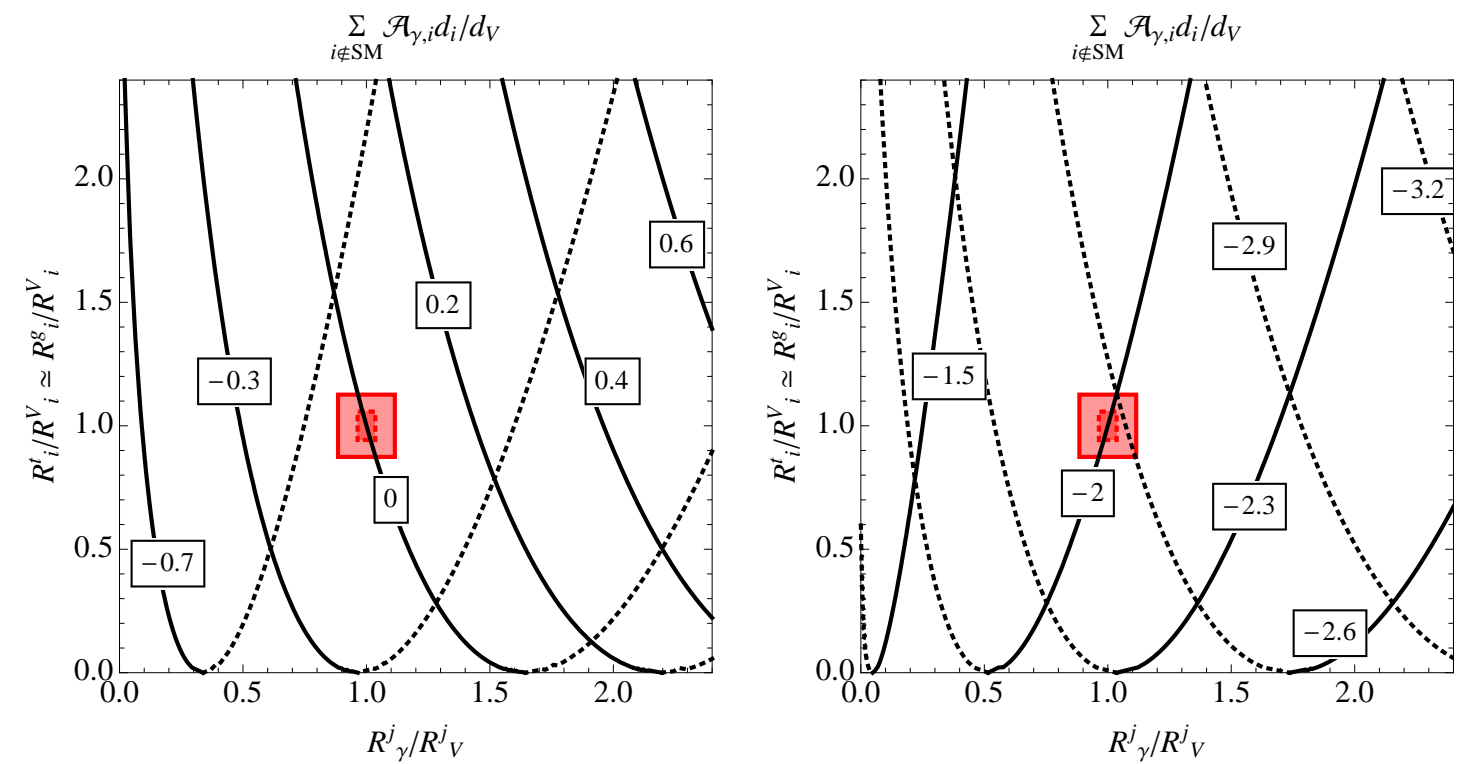

Figure 8.5: Taking the low $\tan \beta$ limit, we plot the new physics contribution to the diphoton rate as a function of $R_{\gamma}^{j} / R_{V}^{j}$ and $R_{i}^{g} / R_{i}^{V}$. The left (right) panel shows values of $\sum_{\mathrm{i} \notin \mathrm{SM}} \mathcal{A}_{\gamma, i} d_{i} / d_{V}$ for which $d_{\gamma} / d_{V}$ is positive (negative). In both panels, solid (dotted) lines show values of $\sum_{\mathrm{i} \notin \mathrm{SM}} \mathcal{A}_{\gamma, i} d_{i} / d_{V}$ for which $d_{g} / d_{V}$ is positive (negative). The red shaded regions are as in Fig. 8.1. We use the uncertainty in $R_{i}^{g} / R_{i}^{V}$ rather than $R_{i}^{t} / R_{i}^{V}$ since the former will be much better measured, as shown in Tab. 8.2.

Let us now discuss in more detail how (iii) acts on the $h \rightarrow \gamma \gamma$ rate. As shown in Eq. (8.13), the $W$ loop contribution to the Higgs coupling to photons destructively interferes with but dominates over the top and bottom quark loop contributions. Regardless of whether the new particles $i$ are scalars or fermions, they have the greatest effect if their loop contributions constructively interfere with the $W$ loop. Thus, large negative values of the new $d_{i}$ will lead to the largest enhancements. The spin of a new particle $i$ does play an important role in the Higgs coupling to photons, however, because the quantities $\mathcal{A}_{\gamma, i}$ asymptote towards beta function coefficients in the large $m_{i}$ limit. A Dirac fermion $\psi$ contributes more strongly to running than a complex scalar $\phi$ of the same quantum numbers, since $b_{1 / 2}=4 b_{0}$, and we find that 
for large masses

$$
4 \eta_{I, \phi}=\eta_{I, \psi}
$$

which signifies that for a fermion and a scalar with equal masses, the scalar must couple 4 times more strongly to the Higgs in order to account for the same effect on $h \rightarrow \gamma \gamma$ as the fermion. Equivalently, for a fermion and a scalar with the same coupling to the Higgs boson, the scalar must be one quarter as massive in order to accommodate the same effect on $h \rightarrow \gamma \gamma$ as the fermion.

We will henceforth assume the existence of a single, unit charged fermion $\psi$ with a mass that satisfies $2 m_{\psi} \gg m_{h}$, so that its corresponding loop coefficient $\mathcal{A}_{\gamma, \psi}$ is fixed by the beta function coefficient of the new particle, $\mathcal{A}_{\gamma, \psi} \rightarrow b_{1 / 2}=4 / 3$. We treat $\eta_{I, \psi}$ as a free parameter which can vary independently of $m_{\psi}$. As noted earlier, only keeping renormalizable terms in the action typically suggests a range $\left|\eta_{I, \psi}\right| \lesssim 1$ if the new particle is a fermion. The ratio of $R_{\gamma}^{j} / R_{V}^{j}$, including the effects of mixing

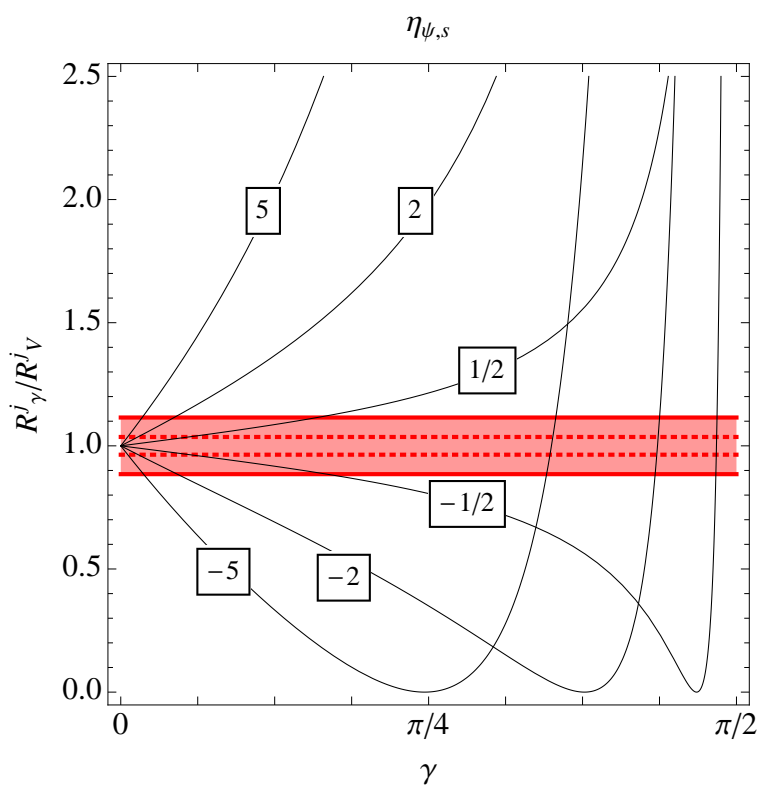

Figure 8.6: Contours of $\eta_{s, \psi}$ as a function of $\left(R_{\gamma}^{j} / R_{V}^{j}, \gamma\right)$, in the decoupling limit $\delta=0$. The red shaded regions are as in Fig. 8.1. 
and a single unit-charged fermion is approximately

$$
\begin{aligned}
\frac{R_{\gamma}^{j}}{R_{V}^{j}} \simeq \mid 1-\left(0.282 \cot \beta-6.14 \times 10^{-3} \tan \beta\right) \tan \delta \\
-\left.0.204 \frac{\sin (\beta+\delta) \eta_{u, \psi}+\cos (\beta+\delta) \eta_{d, \psi}-\tan \gamma \eta_{s, \psi}}{\cos \delta}\right|^{2}
\end{aligned}
$$

To get a sense for the effect of charged particles we consider the decoupling limit, $\delta \rightarrow 0$, and we assume that the coupling goes only through the singlet, so that Eq. (8.33) vastly simplifies to $R_{\gamma}^{j} / R_{V}^{j} \simeq\left|1+0.204 \tan \gamma \eta_{s, \psi}\right|^{2}$, which is depicted in Fig. 8.6. We see that $R_{\gamma}^{j} / R_{V}^{j}$ is more sensitive to the values of the mixing angles for larger magnitudes of $\eta_{s, \psi}$, which nicely illustrates the interplay between the tree-level mixing and loop-level coupling effects: we see here that ramping up the tree-level effect of the mixing angle is ineffective unless the coupling of the loop particle is also appreciable. Without this combined effect, the mixing angles must be very large in order to produce sizable effects on Higgs properties.

We now examine in greater depth the interplay of the mixing angles with the loop effects in Figs. 8.7-8.8, which depict contours of $R_{\gamma}^{j} / R_{V}^{j}$, as shown in Eq. (8.33). The features in these plots are determined by the functional relationships in Eq. (8.33), but we see a few broad patterns. As noted before, $\mathcal{O}(0.3) \lesssim R_{\gamma}^{j} / R_{V}^{j} \lesssim \mathcal{O}(3)$ obtains in the vast majority of parameter space. This is expected because the $W$ boson loop dominates the Higgs coupling to photons, so that $R_{\gamma}^{j}$ is constrained to remain within a factor of a few of $R_{V}^{j}$ unless some cancellations occur. To achieve the largest possible separation of these rates we require one of two possibilities.

- The Higgs couples substantially to new charged particles. The ratio $R_{\gamma}^{j} / R_{V}^{j}$ is enhanced when the new particle interferes constructively with the $W$ loop contribution, which occurs for the largest positive values of the product $\tan \gamma \eta_{s, \psi}$ or for negative (positive) values of $\eta_{u, \psi}$ and $\eta_{d, \psi}$ if $\delta<\pi / 2-\beta$ (if $\delta>\pi / 2-\beta$ ). 
- The Higgs mixes with scalars which substantially modify its coupling to top and bottom quarks. The ratio $R_{\gamma}^{j} / R_{V}^{j}$ is enhanced (suppressed) if $\delta<0(\delta>0)$. As $\tan \beta$ increases, these effects diminish until the bottom contribution becomes important and the effects strengthen again.

These combined effects allow a non-negligible separation of the $\gamma$ and $W$ signal strengths without resorting to extreme values of the couplings or mixing angles.

These two simple phenomena describe most of the broad features we see in Figs. 8.78.8. However, a few other important features can occur in small slices of parameter space where certain Higgs rates go to zero: for example, due to vanishing Higgs couplings to photons (at special values of the mixing angles or the couplings), to the massive electroweak vector bosons (at $\delta \rightarrow \pm \pi / 2$ ), or to all SM particles (at $\gamma \rightarrow \pm \pi / 2$ ). These limits cause $R_{\gamma}^{j} / R_{V}^{j}$ to either diverge or vanish, thus deviating greatly from the general correlation $R_{\gamma}^{j} / R_{V}^{j} \sim \mathcal{O}(1)$. We begin with Fig. 8.7, where we see the dependence of $R_{\gamma}^{j} / R_{V}^{j}$ as a function of $(\delta, \gamma)$ given a single, unit-charged fermion that couples exclusively to $\phi_{s}$ with strength $\eta_{s, \psi}=1$. The Higgs coupling to SM particles will vanish as $\gamma \simeq \pm \pi / 2$, but the Higgs couplings to photons will still be mediated in this case by particles that couple to the $\phi_{s}$. Thus as long as $\eta_{s, \psi} \neq 0$, then as we approach $\gamma \simeq \pm \pi / 2, R_{V}^{j}$ will decrease faster than $R_{\gamma}^{j}$, and the ratio in Eq. (8.33) diverges. As we approach $\gamma \simeq-\pi / 2$, however, there is a special intermediate value of $\tan \gamma$ below which all the contours are tightly packed, which occurs because the Higgs coupling to photons vanishes but the coupling to the massive electroweak vector bosons does not. The photon coupling can only vanish if the new charged particle causes total destructive interference in the photon loop. If $\eta_{s, \psi}<0$ we would find the clustering at positive values $\tan \gamma$ where the singlet contribution could again interfere destructively with the $W$ loop. In Fig. 8.7, there is also a "pinching" feature at the corners of the plot near the anti-coupling limit, $\delta \simeq \pm \pi / 2$. These features arise because at those points the Higgs couples neither to photons nor to vectors. 


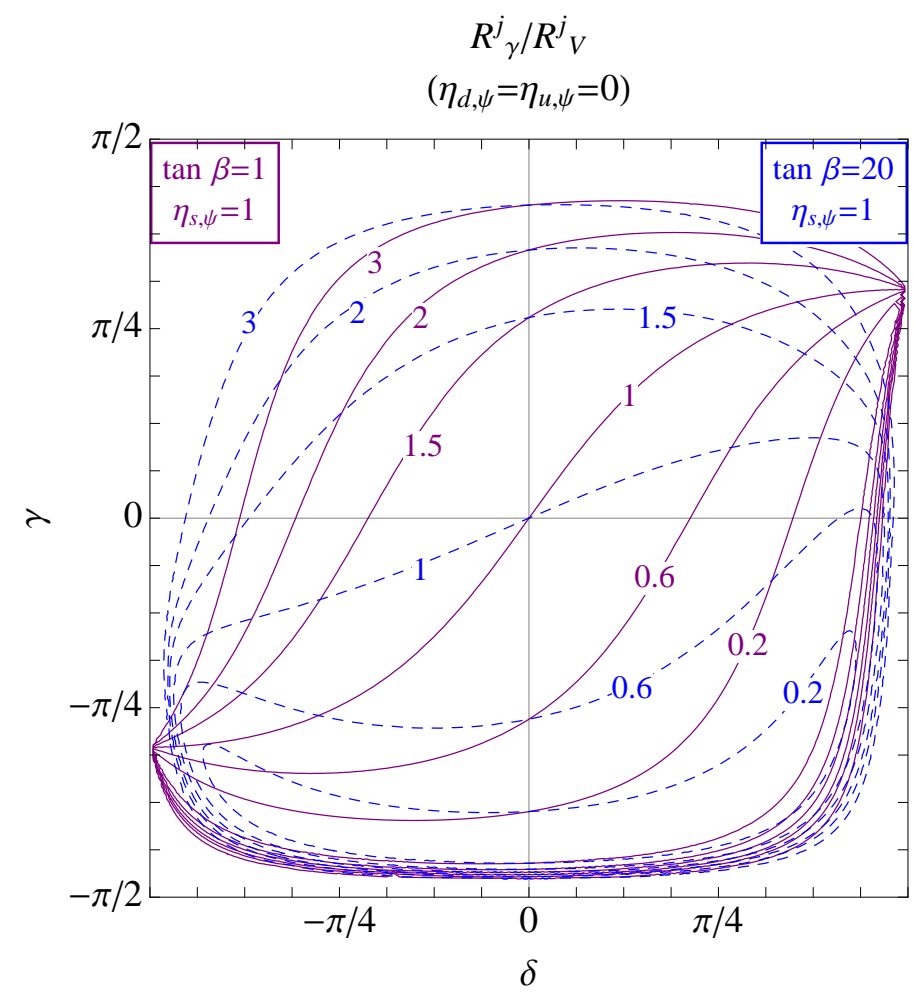

Figure 8.7: Contours of $R_{\gamma}^{j} / R_{V}^{j}$ as a function of $(\delta, \gamma)$ for fixed values of $\tan \beta$ and $\eta_{s, \psi}$ for a unit-charged fermion, and $\eta_{d, \psi}=\eta_{u, \psi}=0$.

We see very similar behavior in Fig. 8.8, where we again consider a single unitcharged fermion which couples to the $\phi_{s}$ component of the Higgs. The clustering and pinching behaviors as $\delta \rightarrow \pm \pi / 2$ are precisely analogous to those described in Fig. 8.7. The behavior of $R_{\gamma}^{j} / R_{V}^{j}$ is in fact symmetric in $\eta_{s, \psi}$ and $\tan \gamma$ because this ratio only depends on the product $\tan \gamma \eta_{s, \psi}$-the plots are visibly different only because we have plotted $\gamma$ as the axis in Fig. 8.7 rather than $\tan \gamma$.

We also have chosen discrete values of $\gamma=0.2,0.4$. As follows from the general discussion and as shown in Fig. 8.6, we see that for larger mixing angles $\gamma$ the diphoton rate is more sensitive to $\eta_{s, \psi}$ because the new charged particle can now be more strongly coupled to the physical Higgs. In the same way, the $\delta$ dependence is sensitive to the value of $\beta$. Because the top quark loop destructively interferes with the $W$ loop we see an enhanced diphoton rate where the $t$ interference is most suppressed this occurs with the smallest $\tan \beta$ and when $\delta<0$. This effect is diminished at larger 

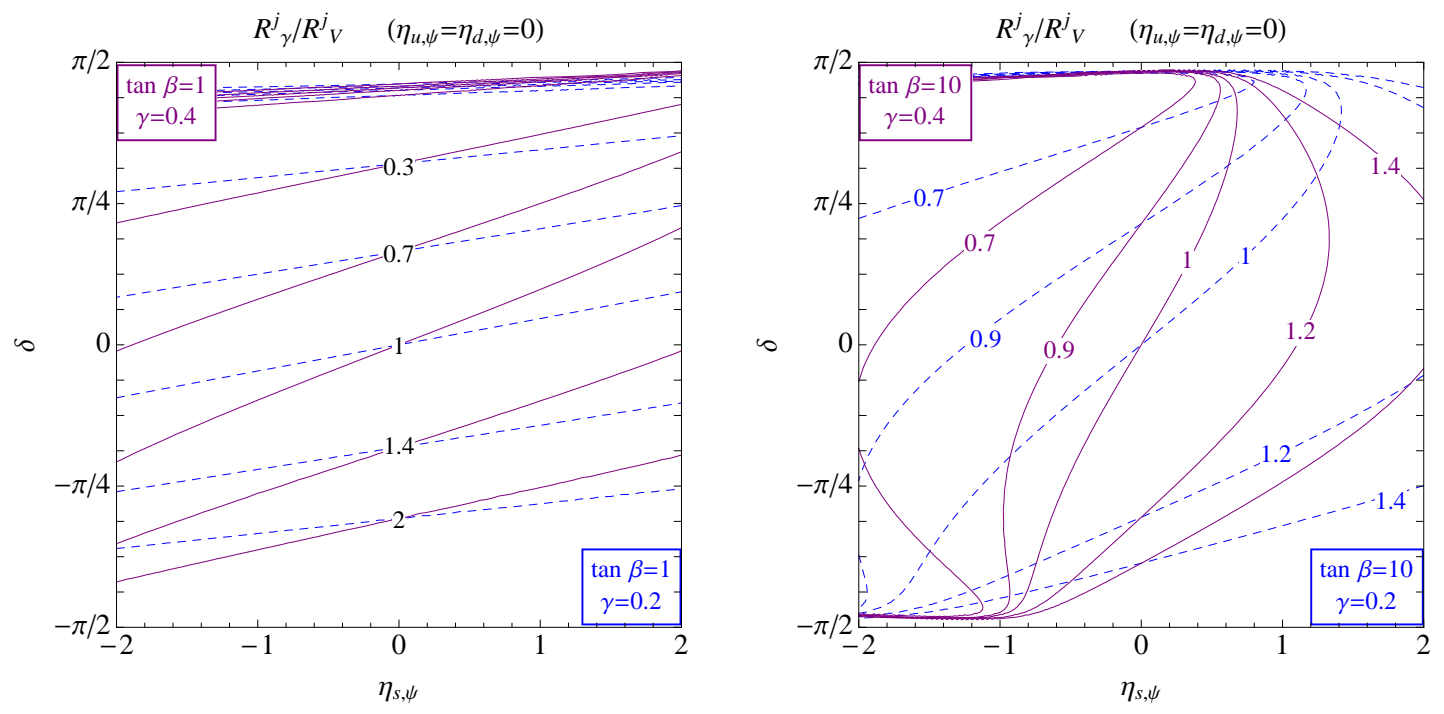

Figure 8.8: Contours of constant $R_{\gamma}^{j} / R_{V}^{j}$ for fixed values of $\tan \beta$ using a single fermion loop particle that couples only to the singlet component of the Higgs. We take $\gamma=0.2(0.4)$, which represents the $68 \%$ (95\%) confidence level limits on the amount of the singlet in the lightest physical Higgs. The pinching behavior should occur in the left panel as well, but requires $\left|\eta_{s, \psi}\right|>2$.

$\tan \beta$ so that the contours are less steeply inclined against the $\delta$ direction and more inclined against $\eta_{s, \psi}$, as is seen by comparing the left and right panels of Fig. 8.8.

\subsection{NMSSM}

The NMSSM is one of the best studied and most well-motivated examples of a singlet extension to the standard 2HDMs. Here we show how our formalism facilitates the analytic extraction of non-SM-like Higgs production and decay. We use the standard MSSM superpotential (retaining the $\mu$ term) enhanced by singlet contribution

$$
W \supset(\mu+\lambda S) H_{u} \cdot H_{d}+\frac{1}{2} \mu_{s} S^{2}+\frac{1}{3} \kappa S^{3} .
$$


We also use standard terminology for the soft SUSY-breaking terms, such that the soft SUSY-breaking Lagrangian contains soft Higgs terms

$$
\begin{aligned}
\mathcal{L}_{\text {soft }} \supset m_{H_{u}}^{2}\left|H_{u}\right|^{2}+m_{H_{d}}^{2}\left|H_{d}\right|^{2}+m_{S}^{2}|S|^{2} & +\left(B \mu H_{u} \cdot H_{d}+\frac{1}{2} B_{s} \mu_{s} S^{2}+\text { h.c. }\right) \\
& +\sqrt{2}\left(A_{\lambda} S H_{u} \cdot H_{d}-\frac{1}{3} A_{\kappa} S^{3}+\text { h.c. }\right) .
\end{aligned}
$$

Mapping the phenomenological considerations of Secs. 8.2 through 8.4 onto the NMSSM parameter space here, the only candidate particles within the NMSSM which could in principle be important for our analysis are: squarks, most importantly the $\widetilde{t}$; sleptons, most importantly the $\widetilde{\tau}$; the charged Higgs, which has very tight direct and indirect search constraints that require its mass to be $\gtrsim \mathcal{O}(300 \mathrm{GeV})$, but which does not automatically decouple in the high mass limit; and the chargino, $\chi^{ \pm}$. The $\widetilde{t}$ 's are colored, which generically induce large differences in the gluon fusion and vector boson fusion production channels which are not observed [3]. More importantly, both the stops and staus require very low masses and very large mass mixings to enhance the Higgs diphoton rate, which can destabilize the vacuum by inducing charge- or color-breaking minima $[46,48,49]$. In general, vacuum stability can also arise from purely electroweak charged or singlet states if one requires very large modifications to Higgs properties [13], or the requirement of thermal dark matter [54]. For more modest deviations from a SM-like Higgs, we can ignore more general questions of vacuum stability in the NMSSM [55] and study the case of the chargino and the charged Higgs.

\subsubsection{Masses And Kinematics}

Next, we focus on changes to Higgs production and decay in the NMSSM. The one loop corrections to $h \rightarrow \gamma \gamma$ will come from the charged Higgs $H^{ \pm}$or the charginos $\chi^{ \pm}$. A multiplet of new physics states of equal charge labeled by $i$ contribute to the 
Higgs coupling to photons with a weight

$$
\eta_{I}=\sum_{i} \eta_{I, i}=\sum_{i} \frac{v}{m_{i}} \frac{\partial m_{i}}{\partial v_{I}}=v \frac{\partial}{\partial v_{I}} \log \operatorname{det} \mathcal{M}
$$

following Eq. (8.6). Here $\mathcal{M}$ is the mass matrix for the particles $i$, so the eigenvalues of $\mathcal{M}^{\dagger} \mathcal{M}$ are $m_{i}^{2}$.

The charged Higgs mass is

$$
m_{H^{ \pm}}^{2}=\left[\left(A_{\lambda}+\frac{\lambda \mu_{s}}{\sqrt{2}}+\frac{\lambda \kappa v_{s}}{2}\right) v_{s}+B \mu\right] \frac{v^{2}}{v_{u} v_{d}}+\frac{v^{2}}{2}\left(\frac{g^{2}}{2}-\lambda^{2}\right)
$$

and the chargino mass mixing matrix is

$$
\mathcal{M}_{\chi^{ \pm}}=\left(\begin{array}{cc}
M_{2} & g v_{u} / \sqrt{2} \\
g v_{d} / \sqrt{2} & \mu_{\mathrm{eff}}
\end{array}\right)
$$

where $M_{2}$ is the wino mass parameter and $\mu_{\mathrm{eff}}=\mu+\lambda v_{s} / \sqrt{2}$. The determinant of this matrix is the product of the two chargino masses, which we will call $\bar{m}^{2}$ :

$$
\left(\bar{m}^{2}\right)^{2}=\left(m_{\chi_{1}^{ \pm}} m_{\chi_{2}^{ \pm}}\right)^{2}=\operatorname{det}\left(\mathcal{M}_{\chi^{ \pm}}^{\dagger} \mathcal{M}_{\chi^{ \pm}}\right)=\left(M_{2} \mu_{\mathrm{eff}}-m_{W}^{2} \sin 2 \beta\right)^{2} .
$$

We will use $\bar{m}^{2}$ to parameterize the chargino loop effects. Current chargino constraints from LEP simply require $m_{\chi_{1}^{ \pm}} \gtrsim 103.5 \mathrm{GeV}$ for generic neutralino masses or $m_{\chi_{1}^{ \pm}} \gtrsim$ $92 \mathrm{GeV}$ for nearly degenerate chargino and neutralino masses [56].

In Fig. 8.9 we show contours of constant $m_{\chi_{1}^{ \pm}}$for realistic fixed values of $\bar{m}$. For $M_{2} \lesssim \bar{m}$, the lightest chargino is predominantly $\widetilde{W}^{ \pm}$so that the $\chi_{1}^{ \pm}$mass grows linearly with $M_{2}$, while the opposite is true for large $M_{2}$ where the lightest chargino is mostly $\widetilde{H}^{ \pm}$. The transition from $\widetilde{W}^{ \pm}$to $\widetilde{H}^{ \pm}$occurs around $\bar{m}$, and at this point the off-diagonal terms in $\mathcal{M}_{\chi^{ \pm}}$become important so that there is increased sensitivity to $\tan \beta$ in this region. Away from this feature in the parameter space the chargino 

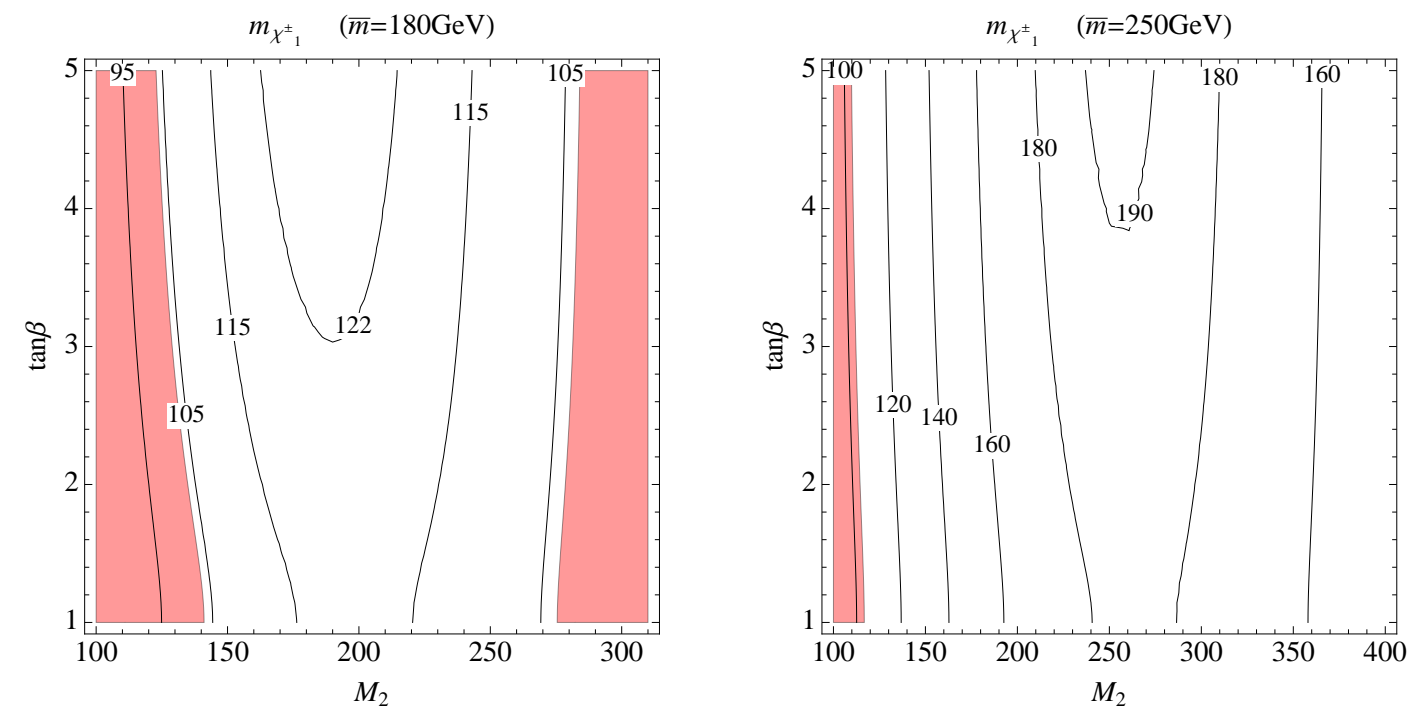

Figure 8.9: Contours of constant $m_{\chi_{1}^{ \pm}}$for fixed $\bar{m}$. Regions that fail LEP bounds are shaded red.

masses are well split so that $m_{\chi_{1,2}^{ \pm}} \sim \mu_{\text {eff }} / \sqrt{2}, M_{2}$ and there is less dependence on $\tan \beta$.

\subsubsection{Signal Strengths}

In Fig. 8.10 we show contours of $R_{V, b}^{g}$ in the $\delta-\gamma$ plane in order to show which values of these parameters give rise to reasonable signal strengths. We take a fixed value of $\tan \beta=1$ since, as shown in Figs. 8.2 and 8.3, the $\beta$ dependence is mild at low $\tan \beta$. Near the origin - which represents the no-mixing, exact decoupling limit - we see that both of the $V$ and $b$ signal strength modifiers are highly sensitive to the departure from the decoupling limit, but not as sensitive to the amount of mixing. This arises because, as noted above, the mixing dependence in $\widehat{R}^{j}$ effectively decouples for small values of the singlet mixing angle since both $\sigma(j j \rightarrow h)$ and $\Gamma_{\text {tot }}$ are proportional to $\cos ^{2} \gamma$. The only $\gamma$ dependence in $R_{V, b}^{j}$ therefore comes from the actual particle couplings, $d_{V, b}$. In contrast, both $\widehat{R}^{j}$ and the couplings carry strong 

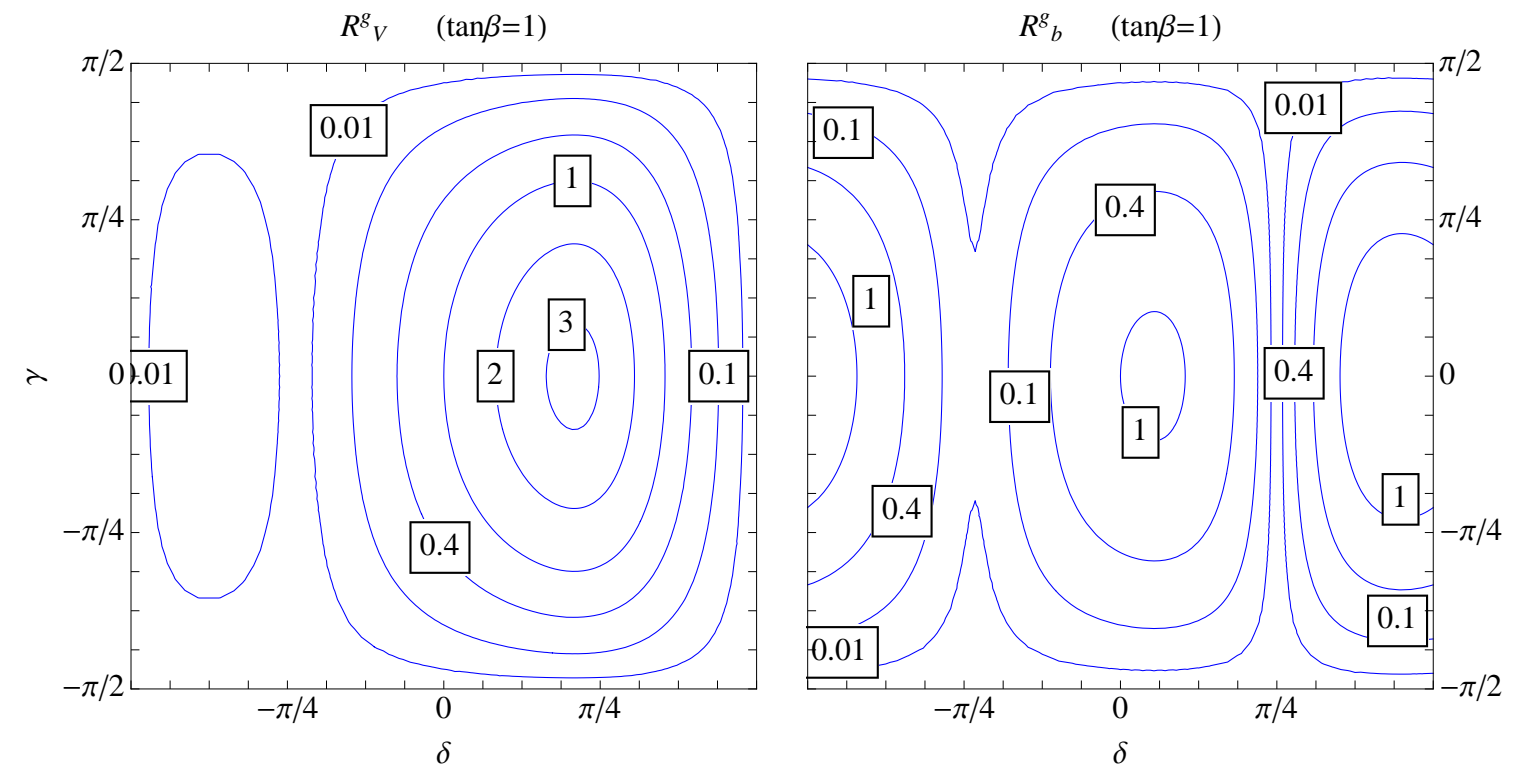

Figure 8.10: Contours of (left) $R_{V}^{g}$ and (right) $R_{b}^{g}$ for $\tan \beta=1$.

sensitivity to $\delta$. Since we observe Higgs decays in rough overall agreement with the SM expectation, we assume that the mixing angles are near the decoupling limit. For simplicity we will take $\delta=0$ in the plots below to guarantee general agreement with the SM values, but we will allow $\gamma$ to vary in order to illustrate the effects on new particles that couple through the singlet.

The Higgs coupling vectors of Eq. (8.5) for the chargino and the charged Higgs are

$$
\begin{aligned}
& \eta_{I, \chi^{ \pm}}= \frac{2 m_{W}^{2}}{\bar{m}^{2}}\left(-\cos \beta,-\sin \beta, \frac{\lambda M_{2}}{\sqrt{2} g m_{W}}\right) \\
& \eta_{I, H^{ \pm}}=\left(\frac{m_{W}^{2}}{m_{H^{ \pm}}^{2}} \frac{1-2 \lambda^{2} / g^{2}}{2 \sin \beta}-\frac{\cos 2 \beta}{2 \sin \beta}, \frac{m_{W}^{2}}{m_{H^{ \pm}}^{2}} \frac{1-2 \lambda^{2} / g^{2}}{2 \cos \beta}+\frac{\cos 2 \beta}{2 \cos \beta},\right. \\
&\left.\frac{2 m_{W}}{g m_{H^{ \pm}}^{2}} \frac{A_{\lambda}+\lambda\left(\kappa v_{s}+\mu_{s} / \sqrt{2}\right)}{\sin 2 \beta}\right) .
\end{aligned}
$$

The constant terms in the $H^{ \pm}$coupling vector ensure that the charged Higgs does 
not automatically decouple even as $m_{H^{ \pm}} \rightarrow \infty$. However, for very large mass, as required by direct and indirect searches, we can make additional statements about the strength of this coupling. We find that $d_{H^{ \pm}}$is proportional to the mixing angle $\delta$ (which tends to be small when $m_{H^{ \pm}}$is large) and to $\cot 2 \beta$ (which goes to 0 as $\tan \beta \rightarrow 1$ ). Combining these scaling arguments with the relative size of the fermion and scalar beta functions $\left(b_{1 / 2}=4 b_{0}\right)$, the $H^{ \pm}$is ineffective compared to the $\chi^{ \pm}$ throughout the bulk of the parameter space we are interested in.

The ratio of the diphoton and vector signal strength modifiers in the NMSSM as a function of the $\chi^{ \pm}$couplings is

$$
\left(\frac{R_{\gamma}^{j}}{R_{V}^{j}}\right)_{\mathrm{NMSSM}} \simeq\left|1+\epsilon(\beta) \tan \delta-\mathcal{A}_{\gamma, \chi^{ \pm}}\left(\frac{2 m_{W}^{2}}{\bar{m}^{2}} \frac{\sin (2 \beta+\delta)}{\cos \delta}+\frac{\sqrt{2} \lambda m_{W} M_{2}}{g \bar{m}^{2}} \frac{\tan \gamma}{\cos \delta}\right)\right|^{2}
$$

From this expression, we see that the photon and vector rates can be different from each other most effectively for large positive $\gamma$, large $\delta$, and small $\tan \beta$. The $\epsilon(\beta)$ piece vanishes in the decoupling limit and the singlet piece does not contribute if there is no mixing, but even in these combined limits the ratio still delivers an increase over the SM prediction by a factor $\sim 4 m_{W}^{2} / 5 \bar{m}^{2} \sim \mathcal{O}(20 \%)$ because of the presence of the additional loop particle. As anticipated above, the couplings $\eta_{I, \chi^{ \pm}}$are not independent of the chargino mass parameters. Interestingly, the sign of this effect works to give an enhancement of $R_{\gamma}^{j}$ as compared to $R_{V}^{j}$.

In Fig. 8.11 we display the values of $R_{\gamma}^{j} / R_{V}^{j}$ resulting from $\chi^{ \pm}$loop effects as a function of $\tan \beta$ and $\lambda M_{2}$, which respectively parameterize the size of the couplings to the doublet and singlet Higgses: $\eta_{u, \chi^{ \pm}} \sim \eta_{d, \chi^{ \pm}} \sim \tan \beta$ and $\lambda M_{2} \sim \eta_{s, \chi^{ \pm}}$. In Fig. 8.12 we show this ratio in the $\lambda M_{2}-\bar{m}$ plane fixing $\tan \beta$ to 1 . In both figures we shade the region for which $m_{\chi_{1}^{ \pm}} \leq 103.5 \mathrm{GeV}$ when $\lambda=0.7$. Across all panels, we see that low $\bar{m}$, low $\tan \beta$ and large $\lambda M_{2}$ drive an enhanced $R_{\gamma}^{j}$ over $R_{V}^{j}$. Within each panel, 

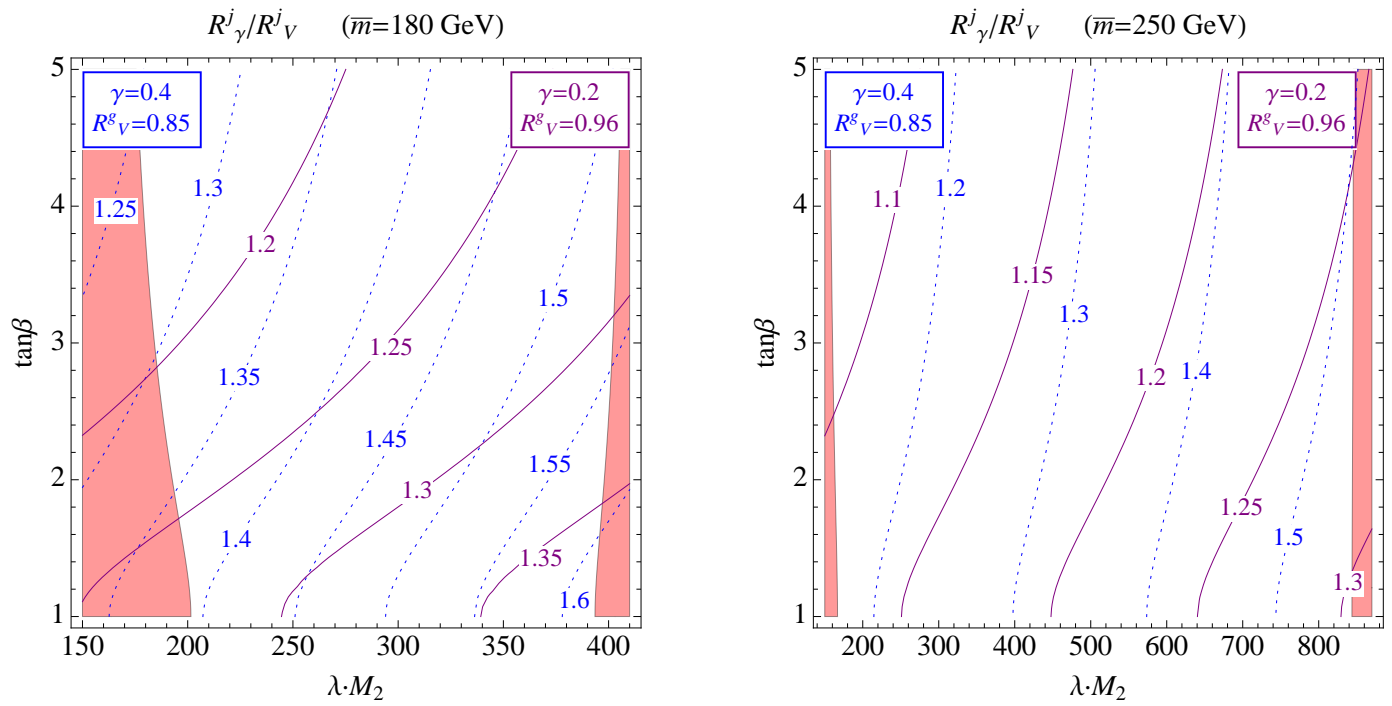

Figure 8.11: Contribution from the $\chi^{ \pm}$loop in the decoupling limit with $\gamma=0.2,0.4$ for (left) $\bar{m}=150 \mathrm{GeV}$ and (right) $\bar{m}=250 \mathrm{GeV}$. In the shaded red regions, $m_{\chi_{1}^{ \pm}} \leq 103.5 \mathrm{GeV}$ for $\lambda=0.7$.
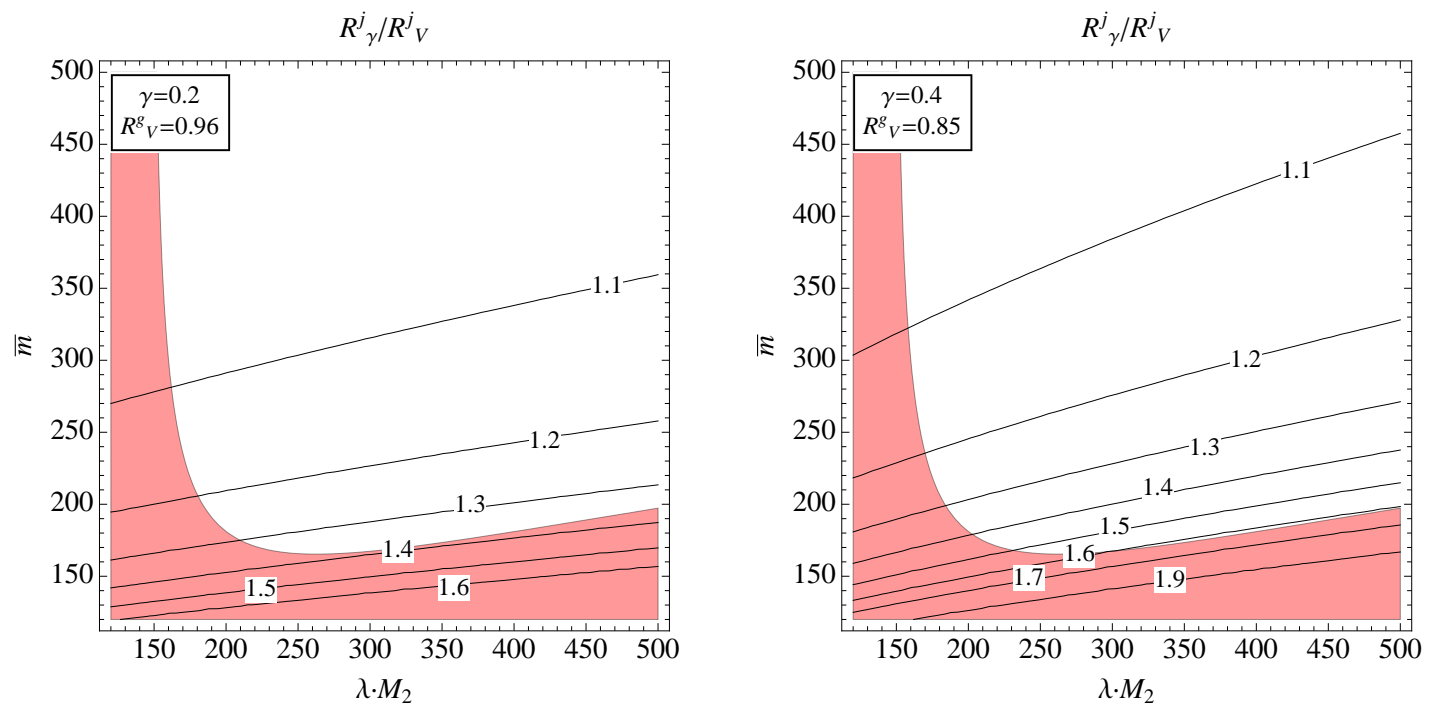

Figure 8.12: Same as Fig. 8.11 but in the $\lambda M_{2}-\bar{m}$ plane and with $\tan \beta=1$.

we see that the rate becomes more sensitive to the singlet coupling as we increase $\gamma$. We also see that $M_{2} \gtrsim \bar{m}$ is preferred, so that $\widetilde{H}^{ \pm}$-like charginos are more effective at 
driving this rate difference. The $\tan \beta$ dependence is also simple to understand, since in the decoupling limit it only enters through the $\sin 2 \beta$ dependence of the doublet Higgs coupling, which is maximized at minimal $\tan \beta$. For instance, in the left panel of Fig. 8.11 (where $\bar{m}=180 \mathrm{GeV}$ ) we see $R_{\gamma}^{j} / R_{V}^{j} \sim 1.6$ around $\lambda M_{2}=380 \mathrm{GeV}$ and $\tan \beta=1$. From Fig. 8.12 we see that $\lambda M_{2} \geq \bar{m}$ leads to the greatest enhancements in $R_{\gamma}^{j} / R_{V}^{j}$, rising up to $R_{\gamma}^{j} / R_{V}^{j} \simeq 1.6$ for the example point picked above.

\subsection{Conclusions}

Higgs boson physics will be a critical component of the ongoing experimental effort at the LHC. While a definitive picture of this newly discovered particle has yet to emerge, experimental errors will shrink, and could offer hints of new dynamics at the electroweak scale in years to come. The focus of this paper has been on theories which have a greater likelihood of being discovered indirectly through modified Higgs properties, as opposed to direct LHC searches. We have investigated a simple parameterization of new physics effects from new electroweak charged and singlet particles which couple to or mix with the Higgs boson. Our formalism has the advantage that it can encompass a number of different electroweak extended models with relatively few parameters. Furthermore, observables such as strength modifiers for various Higgs production and decay modes can be straightforwardly mapped onto this parameter space. These parameters can in turn be easily calculated in any ultraviolet completion consistent with the assumptions of the framework, allowing one to quickly extract their effects on Higgs measurements. For example, we showed how our framework allows one to easily map the NMSSM onto precision Higgs observables in electroweak gauge bosons and $\gamma \gamma$, without making use of parameter scans.

The present work leaves a number of open questions for future study. For example, one could consider how a determination of the mixing angles and loop parameters might be used to constrain the properties of the heavier scalar partners of the Higgs 
boson. Concretely, if the mixing angles for the Higgs boson, $(\alpha, \gamma)$, can be identified by experiment, then unitarity constraints on the scalar mixing angles will in turn restrict the couplings of additional heavy Higgs bosons. One interesting question is whether direct or indirect constraints in these theories will ultimately prove more powerful. Another topic to study further is connecting our formalism for precision Higgs physics to other indirect constraints, e.g. from precision electroweak measurements [57], questions of vacuum stability, and viability of the new electroweak states as dark matter (e.g. as in [54]).

Precision Higgs physics will be an important probe for physics beyond the Standard Model. Should the LHC experiments discover new physics in the Higgs sector, we have presented here a simple roadmap for understanding and parameterizing these effects beyond the SM.

Acknowledgments: SDM and KZ are supported by NSF CAREER award PHY 1049896. KZ is also supported by the DoE under contract de-sc0007859. 


\section{Bibliography}

[1] G. Aad et al. [ATLAS Collaboration], "Observation of a new particle in the search for the Standard Model Higgs boson with the ATLAS detector at the LHC," Phys. Lett. B 716, 1 (2012) arXiv:1207.7214 [hep-ex]. https://twiki.cern.ch/twiki/bin/view/AtlasPublic/HiggsPublicResults

[2] S. Chatrchyan et al. [CMS Collaboration], "Observation of a new boson at a mass of $125 \mathrm{GeV}$ with the CMS experiment at the LHC," Phys. Lett. B 716, 30 (2012) arXiv:1207.7235 [hep-ex]. https://twiki.cern.ch/twiki/bin/view/CMSPublic/PhysicsResultsHIG

[3] G. Aad et al. [ATLAS Collaboration], ATLAS-CONF-2012-170; S. Chatrchyan et al. [CMS Collaboration], CMS-PAS-HIG-12-045.

[4] G. Aad et al. [ATLAS Collaboration], ATLAS-CONF-2012-158; S. Chatrchyan et al. [CMS Collaboration], CMS-PAS-HIG-12-042, CMS-PAS-HIG-12-046.

[5] G. Aad et al. [ATLAS Collaboration], ATLAS-CONF-2012-163, ATLAS-CONF2012-169; S. Chatrchyan et al. [CMS Collaboration], CMS-PAS-HIG-12-041.

[6] G. Aad et al. [ATLAS Collaboration], ATLAS-CONF-2012-161; S. Chatrchyan et al. [CMS Collaboration], CMS-PAS-HIG-12-044.

[7] G. Aad et al. [ATLAS Collaboration], ATLAS-CONF-2012-168; S. Chatrchyan et al. [CMS Collaboration], CMS-PAS-HIG-12-015.

[8] L. J. Hall, D. Pinner and J. T. Ruderman, "A Natural SUSY Higgs Near 126 GeV," JHEP 1204, 131 (2012) arXiv:1112.2703 [hep-ph].

[9] D. Carmi, A. Falkowski, E. Kuflik and T. Volansky, "Interpreting LHC Higgs Results from Natural New Physics Perspective," JHEP 1207, 136 (2012) [arXiv:1202.3144 [hep-ph]]. 
[10] K. Blum, R. T. D'Agnolo and J. Fan, "Natural SUSY Predicts: Higgs Couplings," arXiv:1206.5303 [hep-ph].

[11] V. Barger, M. Ishida and W. -Y. Keung, "Flavor-Tuned 125 GeV SUSY Higgs Boson at the LHC: MSSM and NATURAL SUSY TESTS," arXiv:1207.0779 [hep-ph].

[12] M. Montull and F. Riva, "Higgs discovery: the beginning or the end of natural EWSB?," arXiv:1207.1716 [hep-ph];

[13] N. Arkani-Hamed, K. Blum, R. T. D'Agnolo and J. Fan, '2:1 for Naturalness at the LHC?," arXiv:1207.4482 [hep-ph];

[14] J. R. Espinosa, C. Grojean, V. Sanz and M. Trott, "NSUSY fits," arXiv:1207.7355 [hep-ph].

[15] R. T. D'Agnolo, E. Kuflik and M. Zanetti, "Fitting the Higgs to Natural SUSY," arXiv:1212.1165 [hep-ph].

[16] B. Batell, S. Gori and L. -T. Wang, "Exploring the Higgs Portal with 10/fb at the LHC," JHEP 1206, 172 (2012) arXiv:1112.5180 [hep-ph];

[17] K. Blum and R. T. D'Agnolo, "2 Higgs or not 2 Higgs," Phys. Lett. B 714 (2012) 66 [arXiv:1202.2364 [hep-ph]];

[18] A. Azatov, R. Contino and J. Galloway, "Model-Independent Bounds on a Light Higgs," JHEP 1204 (2012) 127 [arXiv:1202.3415 [hep-ph]];

[19] J.-J. Cao, Z.-X. Heng, J. M. Yang, Y.-M. Zhang, and J.-Y. Zhu, "A SM-like Higgs near $125 \mathrm{GeV}$ in low energy SUSY: a comparative study for MSSM and NMSSM," JHEP 1203 (2012) 086 arXiv:1202.5821 [hep-ph]; 
[20] A. Azatov, R. Contino, D. Del Re, J. Galloway, M. Grassi and S. Rahatlou, "Determining Higgs couplings with a model-independent analysis of $\mathrm{h}$-¿gamma gamma," JHEP 1206, 134 (2012) [arXiv:1204.4817 [hep-ph]];

[21] A. Akeroyd and S. Moretti, "Enhancement of $\mathrm{H}$ to gamma gamma from doubly charged scalars in the Higgs Triplet Model," Phys. Rev. D86 (2012) 035015 arXiv:1206.0535 [hep-ph];

[22] A. Azatov, S. Chang, N. Craig and J. Galloway, "Higgs fits preference for suppressed down-type couplings: Implications for supersymmetry," Phys. Rev. D 86, 075033 (2012) [arXiv:1206.1058 [hep-ph]];

[23] N. Bonne and G. Moreau, "Reproducing the Higgs boson data with vector-like quarks," Phys. Lett. B 717, 409 (2012) [arXiv:1206.3360 [hep-ph]];

[24] W.-F. Chang, J. N. Ng, and J. M. Wu, "Constraints on New Scalars from the LHC 125 GeV Higgs Signal," Phys. Rev. D86 (2012) 033003 arXiv:1206.5047 [hep-ph];

[25] B. Bellazzini, C. Petersson and R. Torre, "Photophilic Higgs from sgoldstino mixing," Phys. Rev. D 86, 033016 (2012) [arXiv:1207.0803 [hep-ph]];

[26] I. Low, J. Lykken and G. Shaughnessy, "Have We Observed the Higgs (Imposter)?," arXiv:1207.1093 [hep-ph];

[27] R. Benbrik, M. Gomez Bock, S. Heinemeyer, O. Stal, G. Weiglein and L. Zeune, "Confronting the MSSM and the NMSSM with the Discovery of a Signal in the two Photon Channel at the LHC," Eur. Phys. J. C 72, 2171 (2012) [arXiv:1207.1096 [hep-ph]];

[28] P. P. Giardino, K. Kannike, M. Raidal and A. Strumia, "Is the resonance at 125 GeV the Higgs boson?," arXiv:1207.1347 [hep-ph]; 
[29] M. R. Buckley and D. Hooper, "Are There Hints of Light Stops in Recent Higgs Search Results?," Phys. Rev. D 86, 075008 (2012) [arXiv:1207.1445 [hep-ph]].

[30] J. Ellis and T. You, "Global Analysis of the Higgs Candidate with Mass 125 GeV," arXiv:1207.1693 [hep-ph];

[31] J. R. Espinosa, C. Grojean, M. Muhlleitner and M. Trott, "First Glimpses at Higgs' face," arXiv:1207.1717 [hep-ph];

[32] D. Carmi, A. Falkowski, E. Kuflik, T. Volansky and J. Zupan, "Higgs After the Discovery: A Status Report," arXiv:1207.1718 [hep-ph];

[33] D. Bertolini and M. McCullough, "The Social Higgs," arXiv:1207.4209 [hep-ph];

[34] A. Joglekar, P. Schwaller and C. E. M. Wagner, "Dark Matter and Enhanced Higgs to diphoton Rate from Vector-like Leptons," arXiv:1207.4235 [hep-ph];

[35] N. Haba, K. Kaneta, Y. Mimura, and R. Takahashi, "Enhancement of Higgs to diphoton decay width in non-perturbative Higgs model," arXiv:1207.5102 [hep$\mathrm{ph}]$;

[36] L. G. Almeida, E. Bertuzzo, P. A. N. Machado and R. Z. Funchal, "Does $H \rightarrow \gamma \gamma$ Taste like vanilla New Physics?," arXiv:1207.5254 [hep-ph];

[37] D. S. M. Alves, P. J. Fox and N. J. Weiner, "Higgs Signals in a Type I 2HDM or with a Sister Higgs," arXiv:1207.5499 [hep-ph].

[38] T. Plehn and M. Rauch, "Higgs Couplings after the Discovery," arXiv:1207.6108 [hep-ph];

[39] J. Kearney, A. Pierce and N. Weiner, "Vectorlike Fermions and Higgs Couplings," arXiv:1207.7062 [hep-ph]; 
[40] T. Kitahara "Vacuum Stability Constraints on the Enhancement of the Higgs to diphoton Rate in the MSSM," arXiv:1208.4792 [hep-ph];

[41] B. A. Dobrescu and J. D. Lykken, "Coupling spans of the Higgs-like boson," arXiv:1210.3342 [hep-ph].

[42] H. Davoudiasl, I. Lewis and E. Ponton, "Electroweak Phase Transition, Higgs diphoton Rate, and New Heavy Fermions," arXiv:1211.3449 [hep-ph];

[43] B. Batell, S. Jung and H. M. Lee, "Singlet Assisted Vacuum Stability and the Higgs to diphoton Rate," arXiv:1211.2449 [hep-ph];

[44] A. Azatov and J. Galloway, "Electroweak Symmetry Breaking and the Higgs Boson: Confronting Theories at Colliders," arXiv:1212.1380 [hep-ph].

[45] M. Carena, I. Low and C. E. M. Wagner, "Implications of a Modified Higgs to diphoton Decay Width,” JHEP 1208, 060 (2012) arXiv:1206.1082 [hep-ph].

[46] M. Carena, S. Gori, N. R. Shah, C. E. M. Wagner and L. -T. Wang, "Light Stau Phenomenology and the Higgs $\gamma \gamma$ Rate," JHEP 1207, 175 (2012) arXiv:1205.5842 [hep-ph].

[47] K. Schmidt-Hoberg and F. Staub, "Enhanced $h \rightarrow \gamma \gamma$ rate in MSSM singlet extensions," JHEP 1210, 195 (2012) arXiv:1208.1683 [hep-ph].

[48] M. Reece, "Vacuum Instabilities with a Wrong-Sign Higgs-Gluon-Gluon Amplitude," arXiv:1208.1765 [hep-ph].

[49] M. Carena, S. Gori, I. Low, N. R. Shah and C. E. M. Wagner, "Vacuum Stability and Higgs Diphoton Decays in the MSSM," arXiv:1211.6136 [hep-ph].

[50] J. R. Ellis, M. K. Gaillard and D. V. Nanopoulos, "A Phenomenological Profile of the Higgs Boson," Nucl. Phys. B 106, 292 (1976). M. A. Shifman, A. I. Vainshtein, M. B. Voloshin and V. I. Zakharov, "Low-Energy Theorems for Higgs 
Boson Couplings to Photons," Sov. J. Nucl. Phys. 30, 711 (1979) [Yad. Fiz. 30, 1368 (1979)].

[51] C. Cheung and Y. Nomura, Phys. Rev. D 86, 015004 (2012) [arXiv:1112.3043 [hep-ph]].

[52] S. Dittmaier, C. Mariotti, G. Passarino, R. Tanaka, S. Alekhin, J. Alwall and E. A. Bagnaschi et al., "Handbook of LHC Higgs Cross Sections: 2. Differential Distributions," arXiv:1201.3084 [hep-ph].

[53] ATLAS collaboration, http: //indico . cern. ch/getFile. py/access?resId=0\&material Id=0\&conf Id=222548.

[54] C. Cheung, M. Papucci and K. M. Zurek, JHEP 1207, 105 (2012) [arXiv:1203.5106 [hep-ph]].

[55] K. Agashe, Y. Cui and R. Franceschini, "Natural Islands for a $125 \mathrm{GeV}$ Higgs in the scale-invariant NMSSM," arXiv:1209.2115 [hep-ph].

[56] LEP SUSY Working group, http://lepsusy.web.cern.ch/lepsusy/.

[57] C. Grojean, E. E. Jenkins, A. V. Manohar and M. Trott, arXiv:1301.2588 [hep$\mathrm{ph}$. 


\section{CHAPTER IX}

\section{Conclusion}

The field of particle physics is at the beginning of an entirely new era. We are gathering more data than ever before with a wide variety of instruments and detectors. With this influx of new data have come some surprises, one very welcome confirmation (in the Higgs discovery), and some disappointments. The goal of the work in this thesis has been to point out some methods for gaining insight into the nature of the physics behind these developments.

The primary focus of my work has been an attempt to understand and characterize the dark sector of the universe. Although we have not irrefutably discovered traces of dark matter in our immediate surroundings, we have convincing evidence of its activity on the very largest scales of the universe. Learning more about the dark matter beyond these large-scale, indirect kinds of evidence is an exciting and even daunting task, and we must efficiently search the entire parameter space that may be available to dark matter.

Exhaustively searching through the entire dark matter parameter space is not feasible, so we need new techniques and new ideas that can use a wide range of supporting evidence to either rule out or highlight the remaining parts of parameter

space. In this way, one fundamental precondition to maximizing our chances of finding dark matter involves pointing out the characteristics that dark matter cannot have. 
Most of my work has been focused on understanding the available data and extending the possible limits ever farther into the parameter space that could have been available to the dark matter. This kind of limit setting is possible on both large and small scales. With a wide variety of collaborators, I have used data ranging from the existence of old pulsars to the energy spectrum of X-ray and gamma-ray photons to place new bounds on dark matter. The ultimate goal of the field is to find the dark matter, to fully characterize all of its properties, and to place it within a complete theory that explains how it relates to the other physics we know. Placing these bounds allows us to narrow our focus in the hunt for dark matter, and is thus a necessary first step in finding this new physics.

Another promising path towards new physics is through the detailed study of the newly discovered Higgs boson. Because of the properties related to how the Higgs can be produced and can decay, it is hoped that these details will give us hints that can point the way to new physics. The Higgs is a particle with entirely different characteristics than those of any particle discovered before, and these new properties imply particular constraints and correlations between its different observable properties. Finding the implications of the constraints and exploiting novel physical mechanisms to illustrate ways around these different constraints can allow us to probe far beyond standard searches for new physics.

The possibility of making contact with hitherto unknowable sectors of the physical universe is now tantalizingly close. We are on the verge of learning incredible new facts about the world as a result of these dark matter searches and Higgs experiments. The community has gained an amazing amount over the years that I have been at Michigan, but, more excitingly, we are now poised to learn even more.

Synthesizing all of these new inputs will require both breadth of knowledge and depth of expertise. Being able to contribute to these open questions and to react quickly to new developments are of equal importance. The work of particle theory 
right now is a balance of pointing towards motivations for new physics and explaining recent discoveries. The search for new physics provides us no guarantees at the start. Discovery requires navigating the path between known and unknown to extract reliable observations and new knowledge from the natural world.

Because we don't know ahead of time what the next discovery will be, we must work to understand what discoveries are possible and what they could mean. Recent experiments have convincingly proven their ability to surprise us with unexpected results. On the other hand, theorists have shown that they are capable of relating many different strands of knowledge in one coherent whole. Once we find something unexpected, we will have to describe it and understand how it connects to the bulk of our physics knowledge, represented by the Standard Model of particle physics. As we look in more detail, our greatest hope is to find connections between the dark and conventional sectors. This will require persistent theoretical, experimental, and observational effort.

In this thesis, I have presented my work related to the search for a theory of dark matter and the search for new physics from a deeper understanding of the Higgs sector. Having the ability to come up with inventive solutions to new observations while also knowing which observations to keep track of are crucial abilities in this new era. I hope this thesis can show that I have been able to contribute a small amount to each field, and will be able to continue doing so as we reach farther towards what is currently unknown. 
APPENDICES 


\section{APPENDIX A}

\section{Appendix: The Dark Matter Inverse Problem: Extracting Particle Physics from Scattering Events}

Here we compile plots for analyses of all five data sets $\left(n_{i}=\right.$ standard, anapole, dipole, $q^{2}$ and $q^{-4}$ ) with equal exposure. Due to space constraints, we show fits to six

of the seven operators $\left(\nu_{i}=\right.$ standard, anapole, dipole, $q^{ \pm 2}$ and $\left.q^{ \pm 4}\right)$ on each page. More comprehensive plots, including plots from data sets with equal event numbers, are shown online [27].

The plots illustrate the qualitative and quantitative features described in Section IV above. Due primarily to the power of the discrimination of the xenon target, analysis that results in three overlapping CLCs will indicate an operator that fits the data well. For low mass DM all operators are effectively indistinguishable in the sense that all analyses result in overlapping CLCs. When the DM candidate is more massive, the distinguishing features of its interaction become more pronounced and harder to mimic, so a particular type of operator is selected.

Within each plot we display CLCs for all three detector elements and all three DM candidate masses, with the exception of the argon contour with the $10 \mathrm{GeV}$ candidate. None of the $10 \mathrm{GeV}$ argon CLCs close because argon sees so few events. 
The argon "exclusion curve" fits comfortably around the xenon and germanium CLCs in all cases. To reduce clutter, these argon curves are omitted from the plots. 

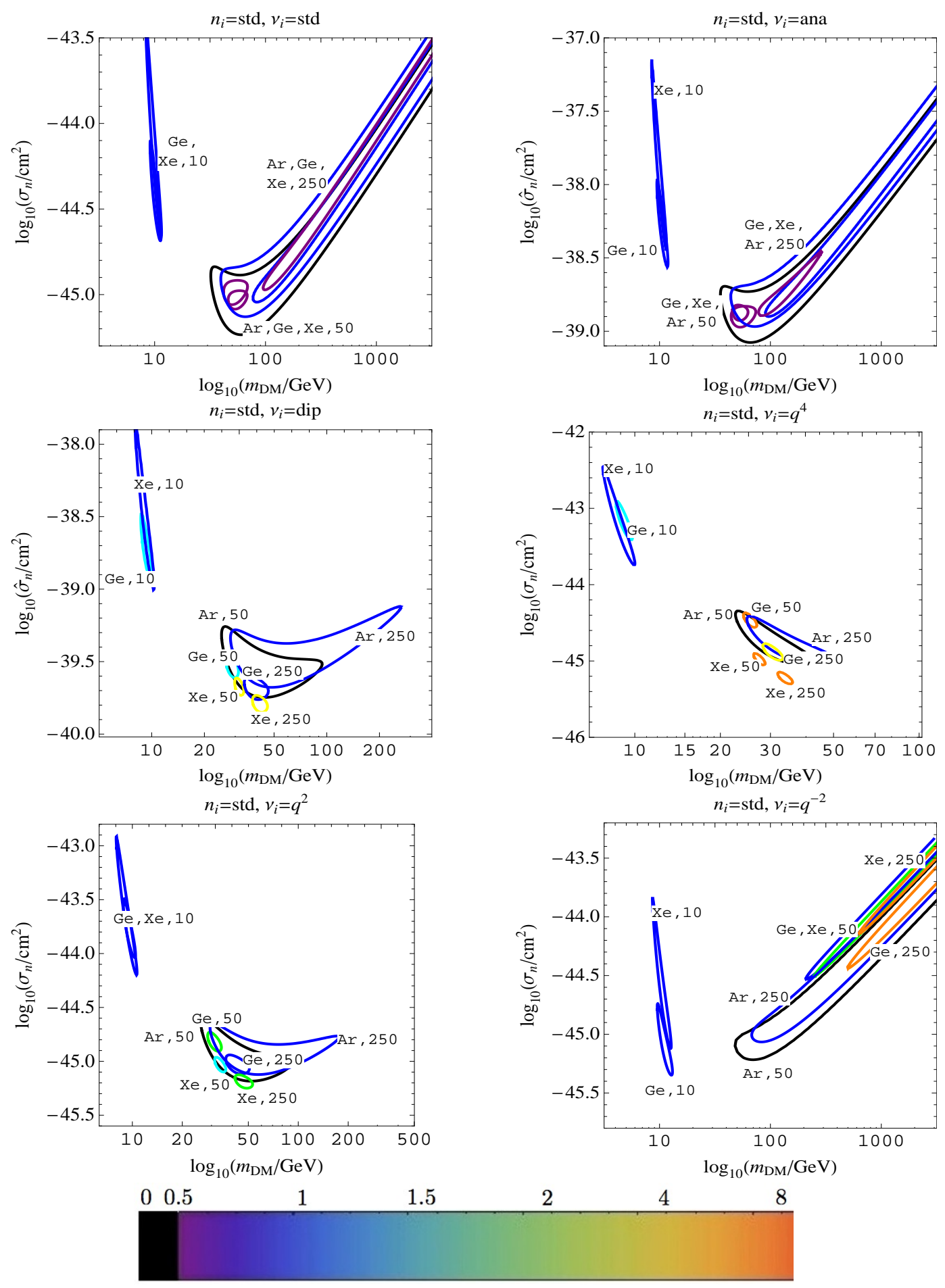

Figure A.1: 95\% CLCs for a 10, 50, and $250 \mathrm{GeV}$ particle interacting through an $n_{i}=$ standard operator. Comparisons are made to $\nu_{i}=$ standard, anapole, dipole, $q^{4}, q^{2}$, and $q^{-2}$ operators. The colors represent the value of $\widetilde{L}_{\min } /$ d.o.f. As described above, cyan and lighter colors correspond to $95 \%$ or worse disagreement with the data. 

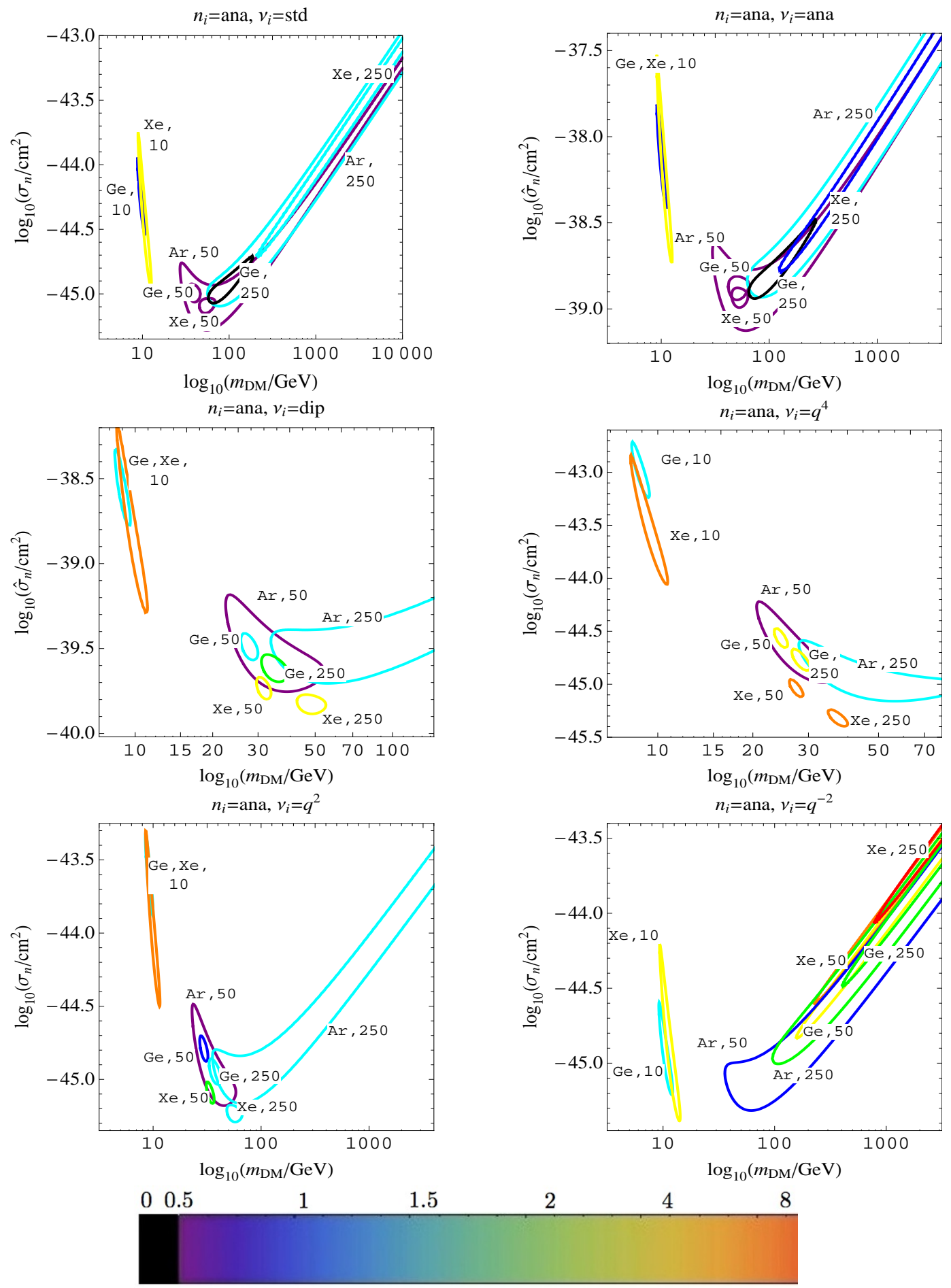

Figure A.2: 95\% CLCs for a 10, 50, and $250 \mathrm{GeV}$ particle interacting through an $n_{i}=$ anapole moment operator. Comparisons are made to $\nu_{i}=$ standard, anapole, dipole, $q^{4}, q^{2}$, and $q^{-2}$ operators. The colors represent the value of $\widetilde{L}_{\text {min }} /$ d.o.f. As described above, cyan and lighter colors correspond to $95 \%$ or worse disagreement with the data. 

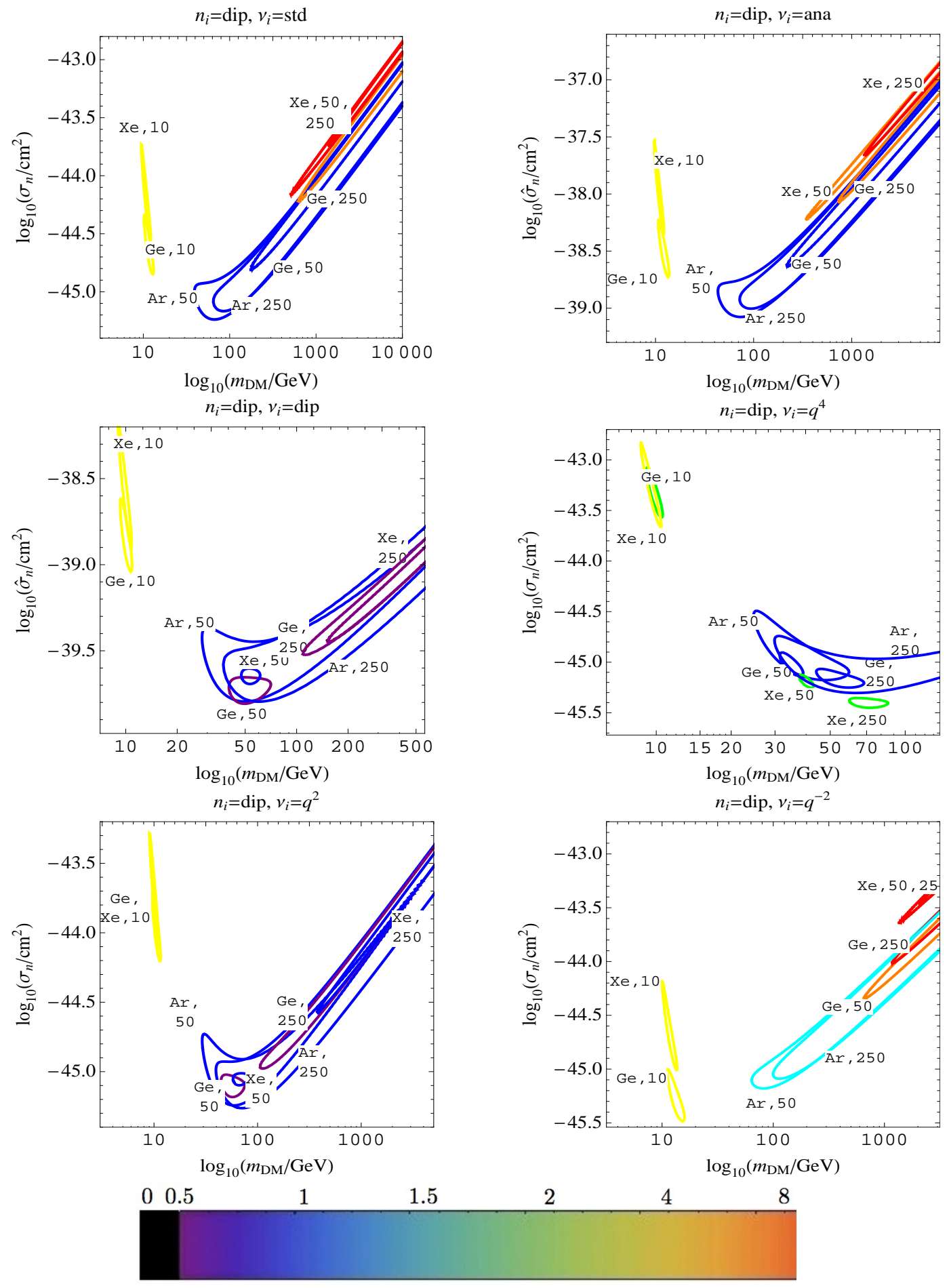

Figure A.3: 95\% CLCs for a 10, 50, and $250 \mathrm{GeV}$ particle interacting through an $n_{i}=$ dipole moment operator. Comparisons are made to $\nu_{i}=$ standard, anapole, dipole, $q^{4}, q^{2}$, and $q^{-2}$ operators. The colors represent the value of $\widetilde{L}_{\min } /$ d.o.f. As described above, cyan and lighter colors correspond to $95 \%$ or worse disagreement with the data. 

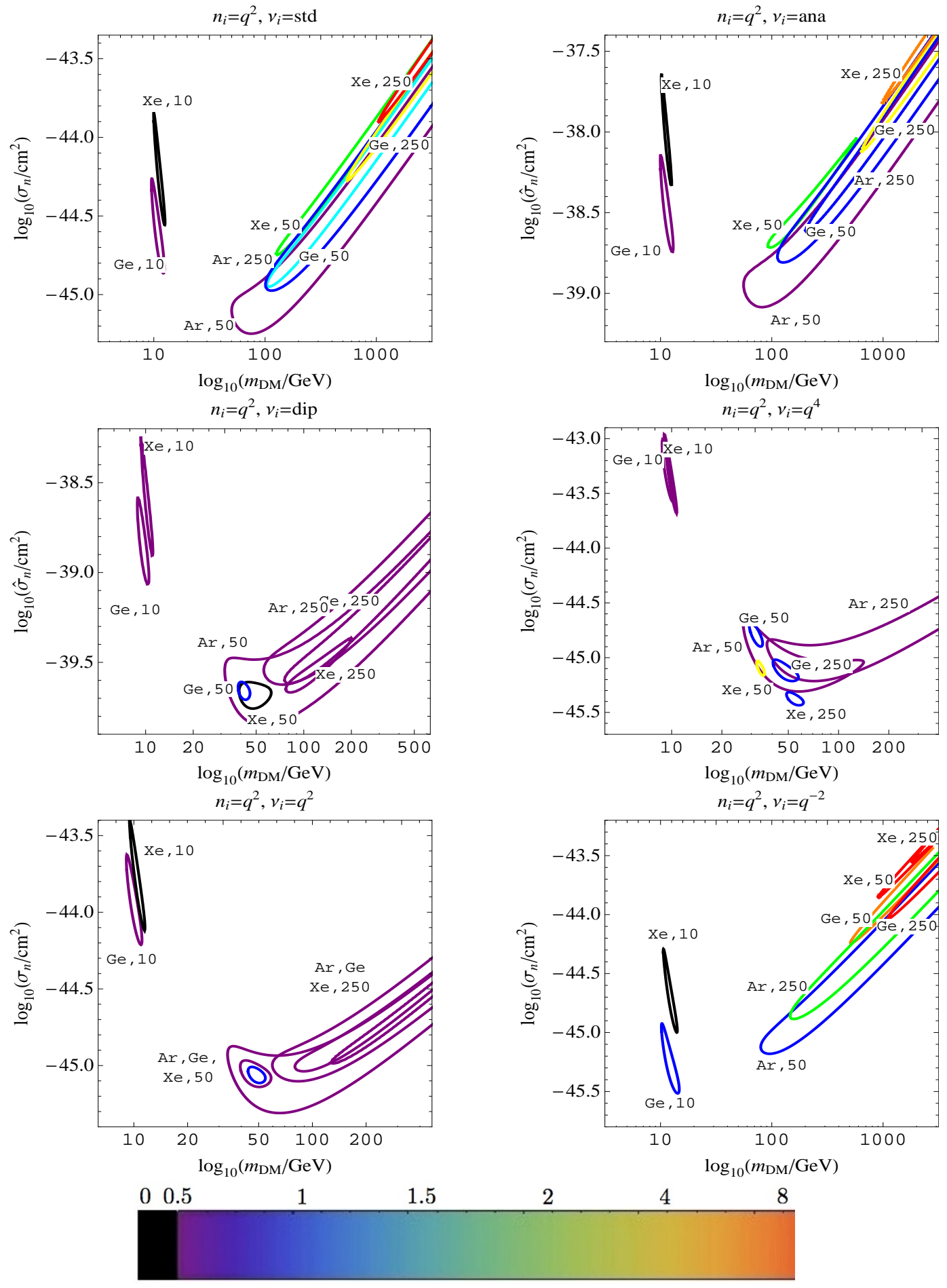

Figure A.4: $95 \%$ CLCs for a 10, 50, and $250 \mathrm{GeV}$ particle interacting through an $n_{i}=q^{2}$ operator. Comparisons are made to $\nu_{i}=$ standard, anapole, dipole, $q^{4}, q^{2}$, and $q^{-2}$ operators. The colors represent the value of $\widetilde{L}_{\min } /$ d.o.f. As described above, cyan and lighter colors correspond to $95 \%$ or worse disagreement with the data. 

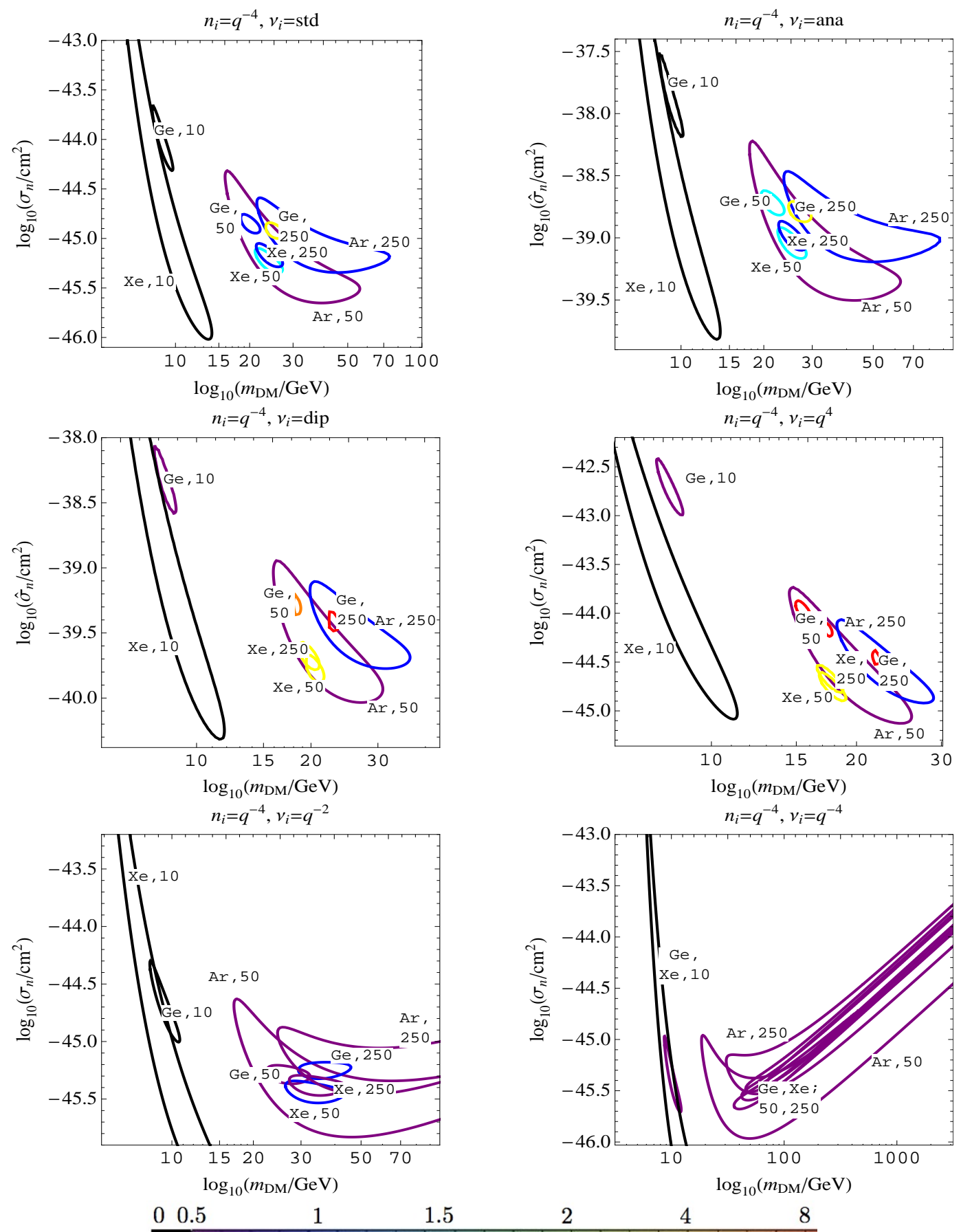

Figure A.5: 95\% CLCs for a 10, 50, and $250 \mathrm{GeV}$ particle interacting through an $n_{i}=q^{-4}$ operator. Comparisons are made to $\nu_{i}=$ standard, anapole, dipole, $q^{4}, q^{-2}$, and $q^{-4}$ operators. The colors represent the value of $\widetilde{L}_{\text {min }} /$ d.o.f. As described above, cyan and lighter colors correspond to $95 \%$ or worse disagreement with the data. 


\section{APPENDIX B}

\section{Appendix: Neutrino Phenomenology in a $3+1+1$ \\ Framework: \\ Fit Details}

We summarize and expand upon the details of our analysis of experimental data included in our fits.

Appearance Experiments: The characteristics of the experiments used in our fits are shown in Table B.1. LSND [2] observed the appearance of $\bar{\nu}_{e}$ with energies $10-60 \mathrm{MeV}$ in a beam of $\bar{\nu}_{\mu}$, consistent with neutrino oscillations that occur in the $\Delta m^{2} \sim 0.2-10 \mathrm{eV}^{2}$ range. $\mathrm{MB}$ also measured $\bar{\nu}_{\mu} \rightarrow \bar{\nu}_{e}$ oscillations [5] with energies $200-3000 \mathrm{MeV}$ consistent with evidence for antineutrino oscillations from LSND. MB did not initially report evidence for oscillations of the form $\nu_{\mu} \rightarrow \nu_{e}[3]$, but an in-depth analysis published after the release of the initial data set supported the interpretation of a low-energy excess consistent with $\nu_{\mu}$ oscillations [4]. We use all of the MB $\nu$ data points in our analysis, including those below $375 \mathrm{GeV}$ which were excluded in the first MB analysis. Due to the low energies of these experiments, we take $a=0$ for our fits. 
We also include the null results of KARMEN [12], E776 [13], NOMAD [14], CCFR [15] and NuTeV [16]. For each null experiment, we find a single data point-that is, the oscillation probability and error for the $L / E$ value-which best matches the $90 \%$ exclusion curves given by the experiments. Due to the generally high energies of these experiments, we use $a=1$ for all experiments except KARMEN in these fits.

\begin{tabular}{l|c|c|c|c|c} 
Experiment & mode & \# points & Distance $(\mathrm{m})$ & $E$ & $\Delta m^{2}\left(\mathrm{eV}^{2}\right)$ \\
\hline \hline MB & $\bar{\nu}_{\mu}, \nu_{\mu}$ & $11 \times 2$ & 541 & $200-3000 \mathrm{MeV}$ & $\gtrsim 0.1$ \\
\hline LSND & $\bar{\nu}_{\mu}$ & 8 & 29.8 & $10-60 \mathrm{MeV}$ & $\gtrsim 0.3$ \\
\hline KARMEN & $\bar{\nu}_{\mu}$ & 1 & 17.7 & $1-50 \mathrm{MeV}$ & $\gtrsim 1$ \\
\hline E776 & $\bar{\nu}_{\mu}, \nu_{\mu}$ & 1 & 1000 & $1-10 \mathrm{GeV}$ & $\gtrsim 1$ \\
\hline NOMAD & $\nu_{\mu}$ & 1 & 625 & $\gtrsim 10-200 \mathrm{GeV}$ & $\gtrsim 10$ \\
\hline NuTeV & $\bar{\nu}_{\mu}, \nu_{\mu}$ & 1 & 1436 & $\gtrsim 10-300 \mathrm{GeV}$ & $\gtrsim 10^{2}$ \\
\hline CCFR & $\nu_{\mu}$ & 1 & 1436 & $\gtrsim 10-300 \mathrm{GeV}$ & $\gtrsim 10^{2}$ \\
\hline \hline TOTAL & $\bar{\nu}_{\mu}, \nu_{\mu}$ & 30 pos., 5 null & $\sim 10-1436$ & $10 \mathrm{MeV}-600 \mathrm{GeV}$ & $\gtrsim 0.1$ \\
\hline
\end{tabular}

Table B.1: Energies, mixings, and mass splitting sensitivities for each appearance experiment.

Disappearance Experiments: The $\nu_{e}$ disappearance constraints include shortbaseline reactor experiments with new reactor flux predictions [17] plus constraints from the ratio of the flux observed in the Bugey $40 \mathrm{~m}$ and $15 \mathrm{~m}$ detectors [18]. ${ }^{1}$ The statistics of the constraint on $\nu_{e}$ disappearance is dominated by the Bugey ratio. Disappearance of $\nu_{\mu}$ is constrained by CDHS [19] and CCFR [20], which we take as single data points, corresponding to the combined oscillation probability for the full energy range. Because both of these experiments search for muon neutrino oscillations between two detectors, very large mass differences are not restricted, since the beam is

\footnotetext{
${ }^{1}$ Even though the new reactor flux has been reported to reflect oscillations of a single sterile neutrino, we find that it is not consistent with our LSND and MB preferred region for the light sterile neutrino, and we use the reactor data as a constraint. On the other hand, it may be fit well with the fifth neutrino of the $3+1+1$ scenario, as shown in Sec. 5.3.1.
} 
likely to be fully oscillated as it arrives at both the near and the far detector. Also, because of the baselines and energies of these experiments, it is a good approximation to ignore the probability of oscillation through $n_{1,2,3}$. We take $a=1$ for CCFR and CDHS, but have $a=0$ for the reactor experiments.

\begin{tabular}{l|c|c|c|c|c} 
Experiment & mode & \# points & Distance $(\mathrm{m})$ & $E$ & $\Delta m^{2}\left(\mathrm{eV}^{2}\right)$ \\
\hline \hline CCFR & $\nu_{\mu}$ & 1 & 714 and 1116 & $40-200 \mathrm{GeV}$ & $10-10^{3}$ \\
\hline CDHS & $\nu_{\mu}$ & 1 & 130 and 885 & $2-6 \mathrm{GeV}$ & $10^{-1}-10$ \\
\hline Mention et al. & $\bar{\nu}_{e}$ & 21 & $9-1050$ & $\sim 3 \mathrm{MeV}$ & $10^{-2}-10^{-1}$ \\
\hline Bugey 40/15 ratio & $\bar{\nu}_{e}$ & 25 & 15 and 40 & $3-8 \mathrm{MeV}$ & $\gtrsim 10^{-2}$ \\
\hline \hline TOTAL & $\bar{\nu}_{e}, \nu_{\mu}$ & 48 & $10-10^{3}$ & $3 \mathrm{MeV}-200 \mathrm{GeV}$ & $10^{-4}-10^{3}$ \\
\hline
\end{tabular}

Table B.2: Energies, mixings, and mass splitting sensitivities for each disappearance experiment.

Unitarity Constraints: The condition for unitarity in the neutrino mass mixing matrix is $U^{\dagger} U=1$, or in component form $\sum_{i} U_{\alpha i} U_{\beta i}^{*}=\delta_{\alpha \beta}$. In practice, this means that sum of the norms of any single row or column in the mixing matrix must equal 1, which bounds the size of any particular element of the matrix. In this way, high confidence measurements of mixing angles for $\nu_{e}$ and $\nu_{\mu}$ with $n_{1}, n_{2}$, and $n_{3}$ can set bounds on the size of the mixings of $\nu_{e}$ and $\nu_{\mu}$ with $n_{4}$ and $n_{5}$.

For instance, solar neutrino experiments such as KamLAND [54] measure $\nu_{e}$ disappearance via the mixing $\sin ^{2} 2 \theta_{\text {sol }}=4\left|U_{e 2}\right|^{2}\left(1-\left|U_{e 2}\right|^{2}-\left|U_{e 3}\right|^{2}-\left|U_{e 4}\right|^{2}-\left|U_{e 5}\right|^{2}\right)$. We can extremize this over the mixing $\left|U_{e 2}\right|^{2}$, but we find that the limits on $\left|U_{e 4}\right|^{2}+\left|U_{e 5}\right|^{2}$ are not very constraining because the solar mixing angle is measured at low confidence and the mixing angle is not maximal. The limits from reactor $\bar{\nu}_{e}$ disappearance experiments provide stronger limits.

On the other hand, a similar analysis is effective in constraining $\left|U_{\mu 4}\right|^{2}$ and $\left|U_{\mu 5}\right|^{2}$ 
from the Super-Kamiokande data, since the atmospheric mixing angle is measured at high confidence to be maximal: a substantial mixing with heavy sterile neutrinos would imply a larger than observed ratio in upward to downward going muon neutrino fluxes. This mixing angle is $\sin ^{2} 2 \theta_{a t m}=4\left|U_{\mu 3}\right|^{2}\left(1-\left|U_{\mu 3}\right|^{2}-\left|U_{\mu 4}\right|^{2}-\left|U_{\mu 5}\right|^{2}\right)$, and extremizing to find the largest value of $\left|U_{\mu 4}\right|^{2}+\left|U_{\mu 5}\right|^{2}$ compatible with the measurements gives

$$
\sin ^{2} 2 \theta_{a t m} \leq\left(1-\left|U_{\mu 4}\right|^{2}-\left|U_{\mu 5}\right|^{2}\right)^{2}
$$

Using the global best fit value for atmospheric mixing angle we find the $90 \%$ (99\%) confidence level constraints:

$$
\left|U_{\mu 4}\right|^{2}+\left|U_{\mu 5}\right|^{2}<0.0175(0.0274)
$$

These bounds on $\left|U_{\mu 4}\right|^{2}+\left|U_{\mu 5}\right|^{2}$ are included in the disappearance constraints, and are in practice the strongest constraints available.

Reactor and Gallium Anomalies: The RAA is detailed in [17,25] and corresponds to a lower-than-expected flux of $\bar{\nu}_{e}$ emitted from nuclear reactors. The GA has been reported in [26] and also discussed clearly in [17] and [55]: SAGE and Gallex have independently measured a lower-than-expected flux of $\nu_{e}$ from the decay of megacurie sources of ${ }^{51} \mathrm{Cr}$ and ${ }^{37} \mathrm{Ar}$, corresponding to anomalously low rates of the reaction $\nu_{e}+{ }^{71} \mathrm{Ga} \rightarrow{ }^{71} \mathrm{Ge}+e^{-}$. This is in principle bounded by similar measurements of the rate of $\nu_{e}+{ }^{12} \mathrm{C} \rightarrow{ }^{12} \mathrm{~N}_{\text {g.s. }}+e^{-}[28,30]$, which are more consistent with expectations [29]. We find that these carbon data are compatible with fits to the RAA and GA data.

We summarize the status of the RAA and GA anomalies as well as the carbon data in Table B.3. In Eq. (5.7) we give our fit to the combined data (using correlation information as reported in [17]). 


\begin{tabular}{l|c|c|c} 
Anomaly & \# points & $\left|U_{e 5}\right|^{2}$ & $\chi_{\min }^{2}$ \\
\hline \hline Gallium & 4 & $0.0708 \pm 0.0317$ & 1.7 \\
\hline Carbon & 11 & $0.0901 \pm 0.0874$ & 8.5 \\
\hline Reactor & 19 & $0.0266 \pm 0.0144$ & 7.2 \\
\hline \hline TOTAL & 34 & $0.0356 \pm 0.0130$ & 19.4 \\
\hline
\end{tabular}

Table B.3: Fits to the reactor, gallium, and carbon anomalies.

Statistical Methods Employed: To place constraints on neutrino mixing from the various null appearance and disappearance experiments we use the raster-scan method described in [56]. For each value of $\Delta m_{41}^{2}$, the $\chi^{2}$ is minimized with respect to the remaining mixing parameters. For the $n$ remaining independent mixing parameters, a $\Delta \chi^{2}$ test is performed to give an $n$-dimensional confidence interval at each $\Delta m_{41}^{2}$. In the $3+1$ scenario, this corresponds to finding 1-dimensional confidence interval for $\sin ^{2} 2 \theta$ at each given value of $\Delta m_{41}^{2}$. The raster-scan provides a more precise confidence region than a global fit. As a result of the sinusoidal dependence of the oscillation probability on $\Delta m_{41}^{2}$, the probability distribution for the $\chi^{2}$ may deviate from gaussian for large deviations from the true value. This may result both in finding an "incorrect" minimum of the $\chi^{2}$ and using an incorrect probability distribution function for determining the sizes of the confidence intervals. By performing a raster scan, one removes the sinusoidal dependence so that the data follow a standard $\chi^{2}$ distribution.

Although this a powerful technique for forming exclusions from null experiments, it is less applicable to cases in which there is a positive result. This is because the raster-scan does not identify preferred values of the parameter $\Delta m_{41}^{2}$. For this reason we perform a global fit to the LSND and MB data, minimizing the $\chi^{2}$ with respect to all parameters. The confidence region is given by a $\Delta \chi^{2}$ for 2 DOF in $3+1$ model and 4 DOF in the $3+1+1$ model. 


\section{APPENDIX C}

\section{Appendix: Neutrino Phenomenology in a $3+1+1$ Framework: Oscillation Formalism}

For nonrelativistic neutrinos whose wavepackets travel with same energy $E$ and whose momenta may be Taylor expanded as $p_{i}=E-m_{i}^{2} / 2 E$, the probability of oscillation to flavor $\nu_{\beta}$ from flavor $\nu_{\alpha}$ is

$$
P_{\nu_{\alpha} \rightarrow \nu_{\beta}}=\sum_{i, j} U_{\alpha i}^{*} U_{\beta i} U_{\alpha j} U_{\beta j}^{*} \exp \left[i\left(m_{i}^{2}-m_{j}^{2}\right) L / 2 E\right]
$$

This formula is easy to evaluate in the limit of many light mass eigenstates because unitarity simplifies the evaluation of the sum. However, the fifth neutrino may either not be accessible or may be produced in a reduced phase space. In this case the evaluation of the sum is less straightforward because the assumption that production processes for all mass eigenstates are similar may no longer be good. With the definitions $\mathbb{U}_{\alpha \beta i j} \equiv U_{\alpha i}^{*} U_{\beta i} U_{\alpha j} U_{\beta j}^{*}$ and $x_{i j} \equiv\left(m_{i}^{2}-m_{j}^{2}\right) L / 4 E$ and taking a phase space 
suppression factor $0<a<1$ on oscillations through $n_{5}$, the oscillation probability is

$$
P_{\nu_{\alpha} \rightarrow \nu_{\beta}}=\sum_{i, j} \mathbb{U}_{\alpha \beta i j} \exp \left(2 i x_{i j}\right)-2 \Re\left[(1-a) \sum_{j} \mathbb{U}_{\alpha \beta 5 j} \exp \left(2 i x_{i j}\right)\right]+(1-a) \mathbb{U}_{\alpha \beta 55} .
$$

Carrying out some standard simplifications allows us to write Eq. (C.2) as

$$
\begin{aligned}
P_{\nu_{\alpha} \rightarrow \nu_{\beta}} & =\delta_{\alpha \beta}\left[1-2(1-a)\left|U_{\alpha 5}\right|\left|U_{\beta 5}\right|\right]+(1-a)\left|U_{\alpha 5}\right|^{2}\left|U_{\beta 5}\right|^{2} \\
& -4 \sum_{5>i>j} \Re\left[\mathbb{U}_{\alpha \beta i j}\right] \sin ^{2} x_{i j}-4 a \sum_{j=1}^{4} \Re\left[\mathbb{U}_{\alpha \beta 5 j}\right] \sin ^{2} x_{5 j} \\
& -2 \sum_{5>i>j} \Im\left[\mathbb{U}_{\alpha \beta i j}\right] \sin 2 x_{i j}-2 a \sum_{j=1}^{4} \Im\left[\mathbb{U}_{\alpha \beta 5 j}\right] \sin 2 x_{5 j}
\end{aligned}
$$

where we use the unitarity condition $\sum_{i} U_{\alpha i} U_{\beta i}^{*}=\delta_{\alpha \beta}$. In the limit $a \rightarrow 1$ we recover the standard result.

In all of the experiments of interest we may ignore oscillations due to the mass differences $\Delta m_{12}^{2}$ and $\Delta m_{23}^{2}$, and Eq. (C.3) simplifies. The oscillation probability of interest in appearance searches such as LSND is found to be

$$
P_{\nu_{e} \rightarrow \nu_{\mu}}=\left|U_{e 4}\right|^{2}\left|U_{\mu 4}\right|^{2}\left\{a\left[(1-r)^{2}+4 r \sin ^{2} \beta\right]+(1-r)^{2}+4 r \sin ^{2}\left(x_{41} \pm \beta\right)\right\}
$$

where $+(-)$ is for $\nu(\bar{\nu})$ oscillations, and the definitions of $r$ and $\beta$ are given in Eq. (5.6) in the text. For disappearance experiments, the relevant formula is

$$
1-P_{\nu_{\alpha} \rightarrow \nu_{\alpha}}=\sin ^{2} 2 \theta_{\alpha 4} \sin ^{2} x_{41}+2\left|U_{\alpha 5}\right|^{2}\left(1-\frac{a+1}{2}\left|U_{\alpha 5}\right|^{2}\right)
$$

where $\sin ^{2} 2 \theta_{\alpha 4}=4\left|U_{\alpha 4}\right|^{2}\left(1-\left|U_{\alpha 4}\right|^{2}-\left|U_{\alpha 5}\right|^{2}\right)$ and we assume that oscillations through $n_{5}$ are averaged. We emphasize that because the phase space factor $a$ only enters at second order in $\left|U_{\alpha 5}\right|^{2}$ the phase space available to $n_{5}$ has very little impact on the predictions and constraints of disappearance experiments. 
The "zero-distance" effect [23] arises because even in the limits $a \rightarrow 0$ and $x_{41} \rightarrow 0$ there remains a nonzero probability for oscillation. Another consequence of the very heavy state is that for fixed $\alpha$ the sum of Eq. (C.3) over $\beta$ betrays nonunitarity. This indicates that we have normalized our states incorrectly. However, this is cancelled by an inverse change in the production and detection cross-sections in the types of experiments considered here [57], so we may use the formulae as if the probabilities were unitary. 


\section{APPENDIX D}

\section{Appendix: Constraining Light Dark Matter with Diffuse X-Ray and Gamma-Ray Observations}

In this paper, we derived robust, conservative constraints by only taking into account the DM signal, as described in Sec. 6.2.3. Stronger constraints can be obtained by fitting the DM signal simultaneously with the different astrophysical background components. This could improve the constraints especially if the DM signal spectrum has a sharp feature like a line or an edge (as appears in an FSR spectrum). However, for softer spectra, while the constraints may be formally stronger, they also suffer from larger systematic uncertainties, since the background components are not known precisely. Furthermore, the isotropic extragalactic flux, which contributes an $\mathcal{O}(1)$ amount to the diffuse galactic signal at high galactic latitudes, can smear out any spectral shapes [107].

To illustrate the improvements possible with using a simultaneous fit of signal and backgrounds, we use the background components as derived by the different collaborations in [63-67] and perform a naïve $\chi^{2}$ goodness-of-fit test (GOF) in Fig. D.1. For the GOF, we take as many distinct background components as have been identified by each collaboration, and, keeping the slopes fixed, allow the normalizations to float. At each point in the $\tau-m_{\text {DM }}$ plane, we add the putative DM signal and minimize the $\chi^{2}$ 
of the background plus the signal with respect to the free normalization parameters. For the HEAO-1 backgrounds, we minimize the $\chi^{2}(S+B)$ by allowing the overall normalization of the broken power law suggested by the collaboration to float. For the INTEGRAL backgrounds, we allow the normalizations of the three smooth background components identified by the collaboration to float independently, and again minimize the $\chi^{2}(S+B)$. These components are a power law with a spectral index $n_{s}=1.55$, a curved component that is the exponential tail of a flat power law (with cutoff around $7.5 \mathrm{keV}$ ), and the smooth diffuse component from extragalactic $e^{+} e^{-}$ annihilation. The COMPTEL collaboration identifies a single smooth background component with index $n_{s}=2.4$, and again we minimize over the normalization of this background. The EGRET and Fermi data are dominated by the systematic error on the effective area, so we take the total shapes as given by the collaborations and allow the normalizations on the entire background shape to float simultaneously. We show the comparison in Fig. D.1, and we find that the GOF improves the constraints, but only by at most an order of magnitude. 


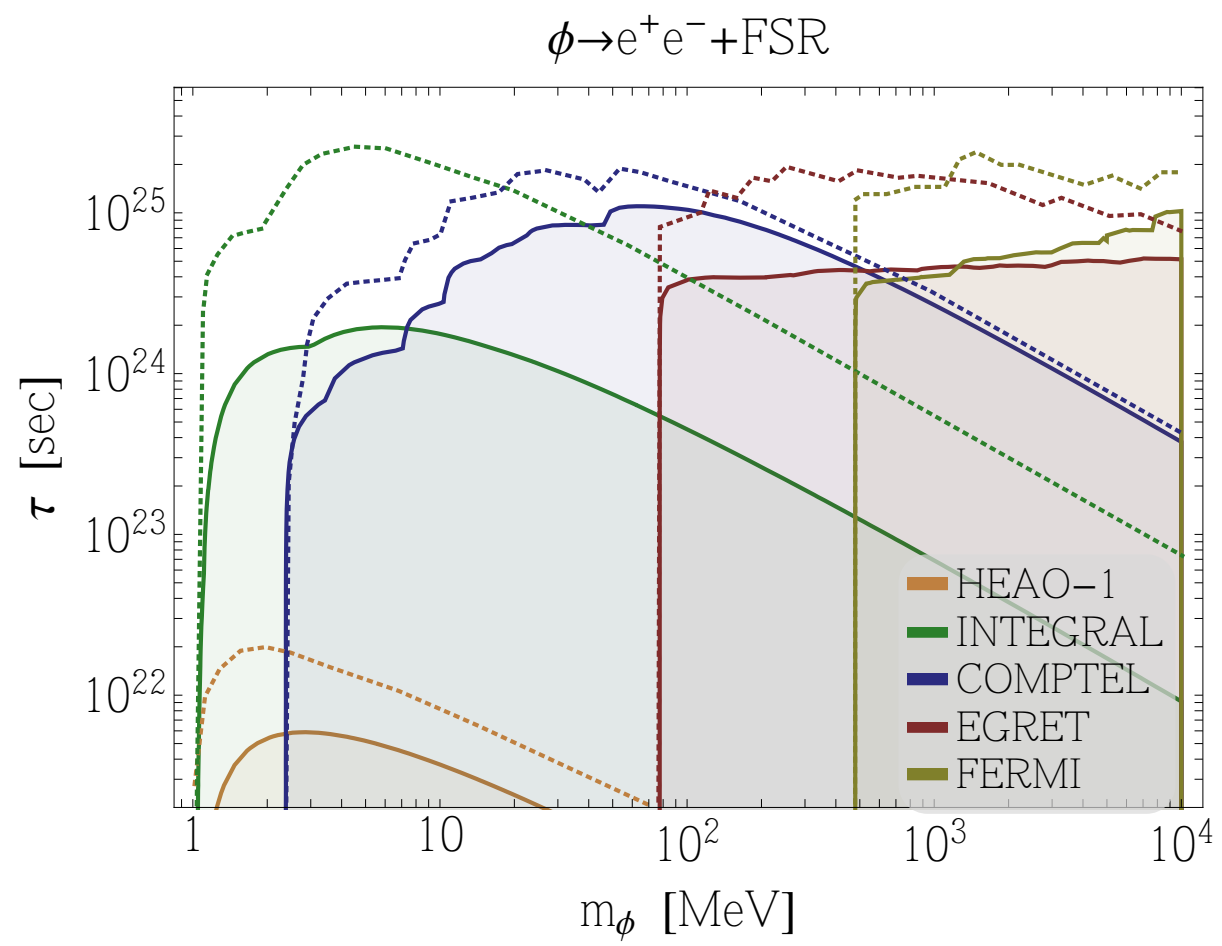

Figure D.1: Comparison of signal-only constraint (solid) and a $\chi^{2}$ goodness-of-fit test (dotted) for each experiment taking the sample spectrum from scalar DM decay to $e^{+} e^{-}$pairs that emit FSR. We show the limits derived from the data described in Sec. 6.2: HEAO-1 (orange), INTEGRAL (green), COMPTEL (blue), EGRET (red), and Fermi (yellow). 